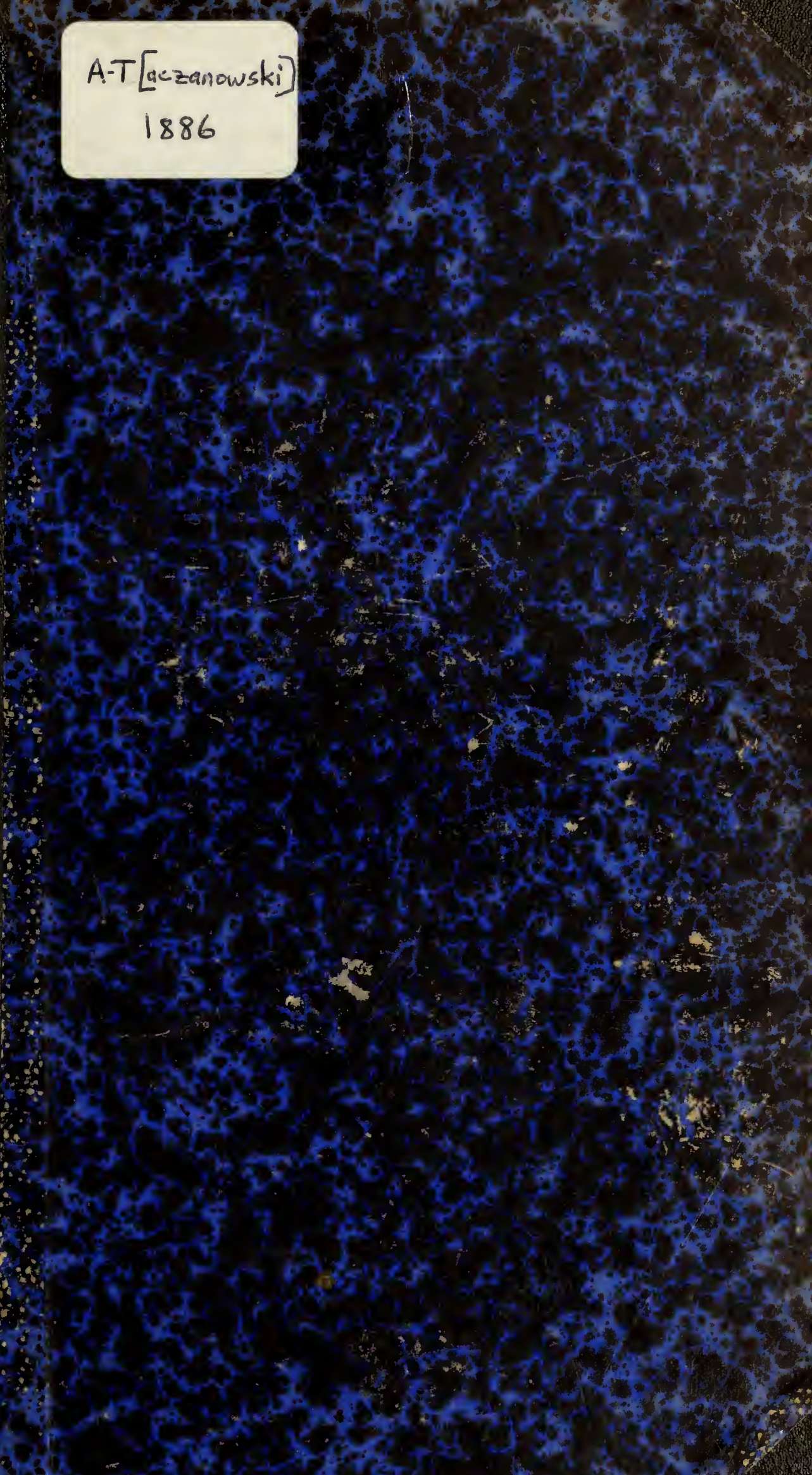





\section{ORNITHOLOGIE}

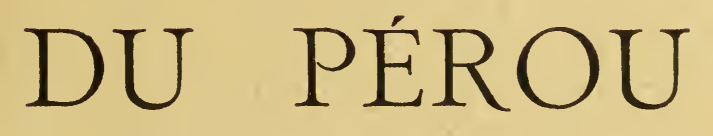

Par Ladislas TACZANOWSKI

\section{TABLES}

TYPOGRAPHIE OBERTHUR, A RENNES

1886

R. Friedländer \& Sohn BUCHHANDLUNG

Berlin N.W., Carlstr. 11. 
Y. I S.8.1.?

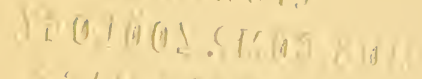

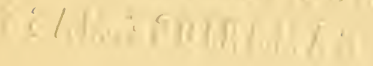


ORNITHOLOGIE

DU PEROU 



\section{ORNITHOLOGIE}

$$
\text { DU PÉROU }
$$

PAR LADISLAS TACZANOWSKI

\section{TABLES}


NOV1 61886

TRANSFESIÍED TO

NUSESUA DF CUMPAKAIIYE ZGOLUEY

$$
\text { May }=2,192 \%
$$




$$
\text { - }
$$





\title{
TABLES SYNOPTIQUES
}

\author{
POUR DETERMINER LES GENREA ET LES ESPĖCES PERUVIENS
}

\section{FAMILLE VULTURID压}

A Tête et cou dénués, sommet de la tête surmonté d'une crête charnue; ailes plus longues que la queue, rémiges secondaires presque égales aux primaires.

A' Doigts externe et interne presque d'égale longueur.

Sarcorhamphus

A" Doigt externe plus long que l'interne.

Cathartes

B Tête lénuće sans crète verticale; queue égalant la moitié de la longueur de l'aile, rémiges prinıares dépassant les secondaires.

b' Queue coupée carrément.

Catilamistes

B" Queue arrondie.

OENOPS

\section{Genre (ENOPS}
$a$ Tète toute rouge.
(OE. ALRA
$b$ 'Tête d'un rouge violâtre, à tache occipitale blanc verdâtre.
(DE. PETATERA
c 'Tète d'un jaune orangé.
(H). ulemtingia

\section{Fanille FALCONIDE}

A Doigts externe et interne réunis au médian par une membrane basale; visage en partic dénué.

$A$ ' Narines oblongues.

Polyborus

$A^{\prime \prime}$ Narines rondes.

A A Coloration uniforme dans les deux sexes. Inxcrer

$A B$ Coloration différente dans les sexes. Mrlvago 
B Doigt interne non réuni au médian par la membrane basale.

l' 'Tarse presque aussi long que les tibias; quatrième ou cinquième rémige la plus longue, queue dépassant beaucoup le bout des ailes.

BA Bec à bords de la mâchoire découpés en deux dents fort prononcées.

Harpagis

$B B$ Bec à bords de la mâchoire découjés en un feston arqué.

lia Une collerette de plumes distinctes des environnantes autour du visage.

Circus

Bb Point de collerette.

lia Face antérieure du tarse réticulée; bec élevé à narines rondes.

Micrastur

Bß Face antérieure du tarse couverte de scutelles.

b1 Doigt externe beaucoup plus court que l'interne.

Geranospiza

B2 Doigt externe beaucoup plus long que l'interne.

Accipiter

b" Tarse beaucoup plus long que les tibias; troisième, quatrieme ou cintuième rémiges les plus longues.

BA Distance entre l'extrémité des rémiges primaires et des secondaires égale ou plus longue que le tarse.

lia Queue égalant le double de la longueur du tarse.

lic Ailes dépassant le bout de la queue.

B1 Doigt médian avec l'ongle plus long que la partie non emplumée du tarse.

B.x Narines ovalaires.

1 Quatrième rémige la plus longue.

BuTEO

2 Troisième et quatrième rémiges égales, doigt médian avec l'ongle plus longs que la partie du tarse couverte de scutelles.

Geranonëtos

3 Troisième, quatrième et cinquième rémiges égales et les plus longues.

B.xx Narines rondes.

LeLcoptenis

Buteola

$B$ ? Doigt médian avec l'ongle plus court que la partie non emplumée du tarse.

Heterospizas 
B $\beta$ Ailes n'atteignant pas l'extrémité de la queue.

Asturina

BB Distance entre l'extrémité des rémiges primaires et des secondaires moins longue que le tarse.

Ba Tête sans huppe.

Ba. Distance entre l'extrémité de l'aile et de la queue plus longue que le pouce.

Buteogallus

D $\beta$ Distance entre l'extrémité des ailes et de la queue plus courte que le pouce.

URUBITING.

Bb Tête huppéc.

$B a$ Narines moins longues que leur distance du sommet du bec.

Harpyia

$B \beta$ Narines plus longues que leur distance du sommet du bec.

BI Queue deux fois aussi longue que le tarse.

H.arpyhaltä̈tus

$B 2$ Quene quatre fois aussi longue que le tarse.

Morphyus

B3 Tarse emplumé jusqu'aux doigts. SPIzä̈тus

$B C$ Tarse réticulé jusqu'aux doigts.

Ba Ongles pleins, non creux en dessous.

Da Doigts armés d'épines, ailes déjassant le bout de la queue.

Panion

$B \beta$ Doirgts sans épines, queue dépassant les ailes.

Gampsonyx

Bb Ongles creux en dessous.

Bu. Queue très longue profondément fourchue.

Nauglerus

$B \beta$ Ailes dépassant l'extrémité de la queue, troisième rémige la plus longue.

ICTINIA

B. Queue dépassant l'aile.

B1 Becélevé, court ; tête grosse couverte au sommet de plumes fines et raides.

Herpetotheres

B2 Bec terminé par un crochet plus ou moins long; pattes courtes. Regerrhunus

D'" Ailes aigüis, à deuxième rémige la plus longue, bords de la màchoire armés d'une dent proéminente.

BA Coloration somblable dans les deux sexes.

lia Taille forte.

FaLco

bl 'laille petite.

Hrpotriorchis

$B B$ Coloration dissemblable dans les deux sexes.

Cerchnets 


\section{GENRE IBYCTER}

a Plumage noir, à base blanche dans la queue. I. ater

$b$ Plumage noir, à ventre, les tibias et les souscaudales blancs.

I. AMERICANUS

\section{Genre MILVAGO}

a Queue du mâle noire terminée de blanc.

M. MEgALOPTERUS

$b$ Queue fauve rayée et terminée longuement de noir.

M. CHimaghima

c Queue fauve grisâtre vermiculée finement de brun, à extrémité longuement brun noirâtre. M. chnsango

\section{Genre CIRCUS}

a Sous-alaires noires.

C. magulosus

$b$ Sous-alaires blanches, chez la $Q$ variées de roux. C. cinereus

\section{Genre MICRASTUR}

a Taille forte.

a' Dessus du corps et des ailes schistacé uniforme.

M. Mirandollei

a" Dessus brun, à collier nucal ocreux.

M. SEMITORQUATUS

6 Taille petite.

b. Quatre raies blanches en travers de la queue. M. giLvicolis

$b$ " Deux raies blanches en travers de la queue. M. Pelzeloni

\section{Genfe ACCIPITER}

a Tout le dessous cendré.

$a$, Sous-alaires rousses.

A. pileatus

$a^{\prime \prime}$ Sous-alaires blanches.

A. BICOLOR

$b$ Dessous blanchâtre, à flancs et poitrine roussâtres; pantalons roux.

A. ERYTHROGNeMis

\section{Genre BUTEO}

a Blanc pur en dessous, dos cendré chez le o", roux chez la $Q$.

B. ERYTHRONOTUS

$b$ 'Tout le dessous isabelle blanchâtre maculé de brun.

B. pennstluanigus 


\section{GeNre ASTURINA}

$a$ Cendré foncé en dessus.

$a$ ' Poitrine cendrée, abdomen blanc rayé de roussâtre ou de cendré.

A. magitirostris

$a$ " Poitrine roussâtre, abdomen rayé de roux et de fauve.

A. Nattereni

$b$ 'Tout le plumage noir, à base de la queue, les sus et sous-caudales blanches.

A. LEUCORrHO I

\section{GeNRE URUBITINGA}

$a$ Noir, à queue traversée d'une bande blanche très large, voisine de la base; sus-caudales blanches.

U. ZONURA

$b$ Brun ardoisé, à queue traversée d'une bande blanche, peu large, plus proche de l'extrémité; sus-caudales schistacées, à bordures blanches. U. SCHISTACEA

$c$ Brun noirâtre, à tibias et le devant de l'aile roux; bas de la queue, sus et sous-caudales blancs.

U. UNICINGTA

\section{GeNre SPIZAETOS}

a Huppe étroite.

S. ORNATUS

$b$ Huppe large.

S. TYRANYUS

\section{GENRE REGERRIINUS}

$a$ Crochet du bec long.

R. MEGARYNCHUS

$b$ Crochet du bec faible.

R. CAYENnENSis

\section{Gexre Falco}

a Tète et dessus du corps noir presque uniforme; cire foncée.

F. DEIROLELCUS

$b$ Dessus du corps noirâtre rayé en travers de cendré; cire jaune.

F. Cassini

\section{GENRE IIYPOTRIORCIIS}

a Tête et dessus du corps noir presque uniforme; cire noire.

H. RUFIGULARIS

$b$ Dessus du corps cendré plombé; sourcil roux très large; cire jaune.

H. FEMORALIS 


\section{FAMILLE STRIGID屟}

A Tête à deux huppes latérales.

I' Fente auriculaire courte, moins large que l'ceil.

1.1 Aile courte, tarse et doigts plus ou moins emplumés.

Bเ во

$A B$ Doigts dénués.

Aa Ailes dépassant l'extrémité de la queue. Scors

Ab Ailes n'atteignant pas l'extrémité de la queue.

L,OPHOSTRIX

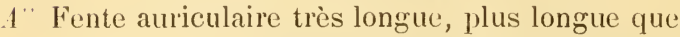
le diametre de l'œil ; ailes atteignant ou dépassant l'extrémité de la queue.

OTus

B Tite sans huppes.

b' Fente auriculaire petite, moins longue que le diamètre de l'œil.

BA Doigt médian avec l'ongle plus long que le tarse.

Glaucidium

BR Doigt médian avec l'ongle plus court que le tar'se.

Pholeoptink

B" Fente auriculaire plus longue que le diamètre de l'œil.

$B A$ Plumes rayonnées courtes au-dessus de l'œil.

$B a$ Doigts emplumés dans leur plus grande moitié basale.

Pulsatrix

$B b$ Doigts nus presque en entier.

Cicc.ABa

$B B$ Plumes rayonnées longues autour des yeux. STtrix

\section{Genre OTUS}

a Huppes longues.

$b$ Huppes très courtes.
O. Mexicanus

O. BRACHYTUS

\section{Genre Ciccaba}

$a$ Dos brun uniforme.

b Dos brun rayé en travers de blanc.
C. MELANONOTA

C. Virgati 


\section{FAMILLE CAPRIMULGIDA}

A Ongle du doigt médian non dentelé; doigt externe à cinq phalanges.

1' Bec élevé, à arête fort arquée, les narines éloignées des plumes frontales; tarse court dénué. S'TEAtтokns

A" Bec fort, aplati, couvert de plumes jusqu'aux narines; tarse court emplumé.

Nictibius

B Ongle du doigt médian dentelé; doigt externe à quatre phalanges.

1)' Base du bec non armée de soies raides.

B.1 Tarse élevé non emplumé, queue médiocre terminée carrément.

BB Tarse court emplumé au-devant et à l'extélieur.

Ba Queue courte coupée carrément.

LUROCALIS

Bb Queue médiocre, échancrée.

Chordeiles

$b^{\prime \prime}$ Base du bec armée de soies raides.

B.1 Queuc normale.

Ba Tarse court emplumé dans sa partie supérieure.

lia Queue arrondic à l'extrémité.

Antrostones

B $\beta$ Queue échancréc ou coupée carrément.

Bb Tarse élevé non emplumé.

STENOpSIS

Nictidronus

BC Queue anornale très longue et profondément fourchue.

HYDROPSALIS

\section{GeNRE NYCIIBIUS}

a Blanchàtre vermiculé de brun, à bande pectorale noire incomplète, taille forte.

N. GRANDIS

$b$ Macules noires sur la poitrine.

b' Brun grisâtre en dessus strié de noir, taille médiocre.

N. Jam.ï̈ENSIS

b" Roux strié et vermiculé de noir, taille médiocre.

N. Lovgicauditus

c Marron foncé vermiculé de noir; poitrine, ventre et scapulaires à taches blanches bordées de noir, taille petite.

N. Bracteatus

\section{Gexre CIORIDEILS}

a Quatre premières rémiges traversées par une large bande blanche. 
a' Sommet de la tête noir maculé de roussâtre, dos noir varié de taches et ocelles gris.

C. aGutipennis

a" Sommet de la tête gris roussâtre varié de grosses stries noires, dos gris clair strić de noir.

C. peruvianus

b Quatre premières rémiges sans bande blanche, les autres primaires à base blanche formant un grand miroir.

C. rupestris

\section{GeNRE ANTROSTONUS}

$a$ Rémiges sans raie blanche.

a' Trois rectrices latérales de chaque cotté de la queue terminées longuement de roussâtre; demi-anneau collaire roussâtre.

A. RuFus

a" Trois rectrices latérales terminées courtement de blanc; plumage roux brunâtre, demianneau collaire blanc, ocelles noirs sur les scapulaires, macules blanches sur l'abdomen. A. ocellatus

a"' Rectrices traversées d'une douzaine de raies roussâtres tachetées de noirâtre.

A. mideulicaudes

$b$ Les deuxième, troisième et quatrième rémiges traversées d'une raie blanche.

b' Deuxième et troisième rectrices terminées de blanc.

A. Nigrescens

b" Première rectrice terminée de blanc.

A. parvulus

\section{GENRE STENOPSIS}

a Trois rectrices latérales de chaque còté de la queue blanches dans le tiers terminal, une bande blanche dans leur moitié, dessin du dessus du corps subtil.

S. BIFASCIATA

$b$ Deux rectrices latérales de chaque côté de la queue terminées de blanc, dessin du dessus du corps gros.

S. equicaudata

\section{GENRE HYDROPSALIS}

a Rectrices externes fort prolongées, atténuées à l'extrèmité, à baguette et barbe externe toutes blanches.

H. SEgMentata

$b$ Rectrice externe fort prolongée noire, turminée de blanc, bordée intérieurement de roux à la base, collier roux.

H. LYRA

c Queue profondément bifurquée, deuxiìme et troisième rectrices blanches terminées de noirâtre.

H. BIFURGATA 


\section{FaMILLE CYPSELIDE}

A Tarses emplumés; doigts externe et médian à trois phalanges; pouce dirigé en avant ou sur le côté; rectrices normales.

a Tarse emplumé, doigts dénués, pouce antérieur. Greselus

$a$ ' Tarse et doigts emplumés, pouce latéral. P.inyptila

B Rectrices terminées en pointe.

$b$ Baguettes à épines proéminentes.

Ch.etura

b' Baguettes à épines non proéminentes.

Crpselö̈nes

\section{Gente CYPSELUS}

a D'un noir fuligineux mat.

$a^{\prime}$ Croupion et un demi-collier blanes. C. Andecolus

$a$ " Plaque jugulaire et une bande le long du dessous blanches.

C. montivagus

$b$ Dessus noir lustré de verdâtre, à plumes bordées finement de blanchâtre.

C. squamatus

\section{Gente CH NTURA}

a. Queue courte, à rectrices ne dépassant les tectrices que par leurs épines.

Ch. poliura

$b$ Queue dépassant plus ou moins les tectrices.

$b$ ' Noir fuligineux, avec un collier roux très large.

Cir. retila

6" Noir luisant en dessus, cendré foncé au croupion et en dessous.

Ch. Sclateri

\section{FAMILLE HIRUNDINIDE}

A Bec robuste, non aplati à la base, à narines rondes. Progne

B Bec aplati à la base, à narines oblongues.

B' Narines voisines des plumes frontales; couleur des parties supérieures du corps à reflet métallique plus ou moins fort.

BA Queue plus ou moins profondément fourchue.

$\mathrm{Ba}$ Sous-caudales concolores à l'abdomen. Hirundo

$B b$ Sous-caudales noires.

Atticon.

BB Queue coupće carrément ou à peine entaillée. 
B' Narines éloignées des plumes frontales; couleur sans reflet métallique sur les parties supérieures du corps.

B.L Barbe externe de la première rénige normale.

\section{Cotyle}

$B B$ Barbe externe de la première rémige à barbules raides recourbées à l'extrémité en crochet.

STELgidopter Y $\mathrm{X}$

\section{GeNRe PROGNE}

a Couleur générale noire lustrée de bleu.

P. purpurea

$b$ Bicolore.

b' Noir lustré de bleu en dessus, blanc en dessous, nébulé de gris au cou et à la poitrine. P'. Chlalyb.aA

b" Gris fuligineux en dessus, dessous blanc, à poitrine grise.

P'. TAPELI

\section{GeNRe HIRUNDO}

a Rectrices externes prolongées en un appendice atténué.

a' Front gris blanchatre; dessous roux. II. erýrhrogastra

$a^{\prime \prime}$ Front et dessous du corps roux. H. Týleri

$b$ Rectrices latérales normales.

$b^{\prime}$ Dessous et croupion blanc's.

ba Dessous blanc pur, une grosse tache alaire

blanche.

H. ALBIVENTRIS

bb Flancs et dessous grisâtres, devant du front blanchatte.

H. LELCORRHOA

lic Poitrine et cotés de l'abdomen enduits de grisâtre, point de tache blanche alaire. H. L.Eccopygu

b" Tout le dessous grisâtre, à gorge d'un gris plus foncé; croupion concolore au dos.

H. ANDICOLA

\section{GeNRE I'TTICORA}

a Dessous du corps blanc, sous-caudales noires. A. cranoleuca

$b$ Dessous du corps noir ou gris.

b' Dessous noir, à bande pectorale blanclıe.

A. FAscista

$b$ " Dessous et croupion gris, tibias blancs.

A. TIBLALIS

b" "Dessous gris uniforme, à sous-caudales noirâtres, croupion concolore au dos.

A. CINEREA

\section{GENRE S'TELGIDOP'TERYX}

a Plumes du croupion concolores au dos.

$b$ Plumes du croupion blanchâtres.
S. BUFICOLLIS

S. IROP'YGIALIS 


\section{F.MILLE TROCHILIDE}

A Queue normale.

I' Oiseaux sans huppe et sans collerette sur les côtés du cou.

A.A Bec à arète dorsale dénudée entre les scutelles nasales, dépassant le bord antérieur des narines, ces dernières découvertes.

Aa Bec plus long que la tête, non cylindrique, rectrices médianes presque égales aux subnédianes.

Aa Bec arqué presque en tiers de cercle, à unàchoire comprimée et creusée sur les côtés jusqu'aux ${ }^{2} / 3$ de sa longueur. Eutoxeres

$A \beta$ Bec légèrement arqué, à mâchoire non comprimée, à sillon creux n'atteignant pas la moitié du bec.

GLiugis

$A b$ Rectrices médianes notablement plus longues que les submédianes, ordinairement allongées et brusquement atténuées après les submédianes, cette partie allongée souvent blanche.

Phä̈thornis

Ac Baguette des rémiges primaires dilatée et aplatie dans sa partie basale.

Aa Queue longue et fourchue profondément.

Eupetomena

$A \beta$ Ailes presque coudées, chez le $\sigma^{x}$ la baguette de la première rémige très dilatée à la base et non barbée à l'extérieur de cette partie, queue non fourchue arrondie.

Cinpylopterus

Ay Ailes falciformes chez le $\sigma^{x}$, à baguette de la première rémige peu dilatée et peu aplatie, barbée à l'extérieur.

Aphantochron

Ad Baguette des rémiges primaires nolmale, queue légèrement arrondie.

LAMPORYIS

Ae Bec droit aussi long que les deux tiers du corps; des plumes squamiformes au front.

Af Bec droit ou peu arqué, moins long que les deux tiers du corps.

Aa Queue tronquée ou presque tronquée. 
A1 Dessous du corps revètu de plumes blanches soyeuses, souscaudales blanches ou cendrées, quelquefois vertes.

1 Rectrices en partie blanches à la base, ou d'un blanc cendré au bout, ou d'un vert uniforme. Leccippus

2 Rectrices sans blanc à la base et à l'extrémité, le blanc du dessous parfois très rétréci. Uranomitra

3 Dessous du corps blanc, à gorge et cou antérieur parsemés de points brillants.

'Thaumatas

A? Dessous du corps revêtu de plumes vertes souvent frangées de blanc, offrant quelquefois une raie longitudinale médiane blanche ne couvrant pas la moitié de la largeur du corps.

Agrrtria

A3 Dessous du corps squamiforme vert; queue subarrondie.

Polytues

Ag Bec élargi à la base graduellement atténué vers l'extrémité.

Aa Dessous du corps en partie roux, souvent d'un vert tendre, rectrices en partie rousses.

Amazilia

$A \beta$ Queue dorée, bronzée ou vert dorée, plus brillante en dessous qu'en dessus.

Chrysuronia

Ay Queue non dorée.

A1 Bec élargi et aplati à la base, menton roux ou tacheté de blanc, cout et poitrine bleus.

Hylogharis

$A 2$ Bec assez large à la base, menton non roux ni blanc, cou et poitrine verts ou d'un vertbleuâtre. Eucephala

A/ Bec aussi long que la tîte.

Droit, terminé en pointe aiguë; ailes atteignant l'extrémité de la queue, doigts courts.

Chlorostilbon

$A B$ Base de la mâchoire couverte de plumes entre les scutelles cachant plus ou moins l'arète dorsale.

Aa Bec plus ou moins arqué, ordinairement fort, tête triangulaire.

$A \alpha$ Bec denticulé à l'extrémité des bords des deux mandibules, région auri- 
culaire parée d'une touffe de plumes se détachant du corps, queue à rectrices larges peu inégales.

Petasophora

$A \beta$ Queue entaillée, à rectrices terminées en angle émoussé.

JOLANA

Ag Queue à rectrices rigides, peu inégales, atténuées vers l'extrémité et aiguës au bout, barbe externe de la rectrice latérale étroite; gorge et devant du cou parés d'une plaque squamiforme verte ou d'un bleu violet.

Oreotrochilus

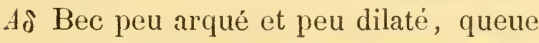
presque tronquée, à rectrices larges subarrondies, gorge et devant du cou métallique non squamiforme.

$A_{\varepsilon}$ Rémige externe atténuée, queue échancrée, à rectrices larges terminées en angle obtus, gorge et poitrine écailleuses.

LAFRESNYA

Ab Bec droit, à base plus ou moins emplumée couvrant les narines; tête triangulaire; queue plus ou moins entaillée. $A \propto$ Bec plus long que la tête et le corps. Docnastes $A \beta$ Bec moins long que le corps.

$A x$ Bec beaucoup plus long que la tête.

A1 Taille très forte, plumage non métallique, queue longue et large.

Florisuga

12 Extrémité du bec courbée légèrement en haut; queue ample et longue, ailes en grande partie bleu métallique.

Pterophanes

A3 Sommet de la tête métallique fort luisant, corps en partic; queue et ailes rousses, une gemme violette au cou du mâle.

Patagona

4 Queue ample faiblement entaillée; plumage métallique, une gemme jugulaire et prase frontale brillants.

Helianthea

Aj Queue longue, fourchue; plumage métallique, tête ornée d'une bande médiane fort brillante.

Clytolama 
A6 Queue ample fort. entaillée; plumage brillant, une large bande pectorale blanche ou rousse, ou plumage général sombre prenant sous certain jour un écrlat étincelant au croupion.

BOURCIERIA

A7 Queue médiocreentaillée; plumage métallique, bande médiane du sommet de la tite et gorge fort brillants; rémiges secondaires en partie rousses. LaMpraster

A8 Queue longue profondément fourchue; plumage métallique, celui du centre du sommet de la tête, gorge et poitrine fort brillants.

HeLjodoxa

Axx Bec presque aussi long que la tete.

A1 Queue large médiocrement entaillée; tarse emplumé; plumage métallique fort étincelant sous certain jour, en partic non métallique.

Panoplites

$A 2$ Queue longue fourchue; plumage métallique, prase frontale et gorge fort brillants.

Helototria

A3 Bec légèrement fléchi; queue large peu entaillée, à baguettes roussâtres dans les rectrices; dessous du corps fauve, une gemme rouge fort brillante au cou.

Pheolema

Ac Bec droit, la tête subarrondie.

$A \propto$ Bec moins long que la moitié du colps.

A 1 Pattes garnies d'une manchette duveteuse, dessous du corps fort itincelant.

Eriognemis

$A 2$ Pattes non pattues, plumage général terne, excepté le croupion qui est largement fort étincelant, une touffe de plumes blanches ou fauves se détachant du corys sur le milieu de l'abdomen.

Aglahatis

$A \beta$ Bec égalant les $2 / 3$ du corps, front paré l'une plaque métallique fort luisante. 
Ad Bec légèrement arqué égral à la moitié du corps, queue échancrée, gorge et cou antéricur parés de plumes squamiformes vertes étendues jusqu'au bord antérieur des yeux, le reste du dessous ordinairement bleu.

Thalurania

Ae Bec droit plus long que la tête.

$A_{\alpha}$ Gorge et cou antérieur parés d'une plaque rouge ou violette très polie. Helangetus

$A \beta$ Gorge et cou antérieur parés de plumes squamiformes vertes.

Urosticte

Af Bec droit égal ou un peu plus long que la tête, fort comprimé à l'extrémité mème.

A @ Queue échancrée métallique sur les deux pages, changeant en bleu ou lilas sur la supérieure.

Metallura

$A \beta$ Queue tronquée, à rectrices peu métalliques terminées d'une tache blanchâtre, gorge blanchâtre parsemée de points submétalliquesor.

Ay Queue tronquée médiocrement métallique, à rectrices terminées par une bordure blanche, sommet de la tête et gorge d'un bleu violet.

Ag Oiseaux ornés d'un rabat jugulaire métallique. très splendide, plus ou moins atténué, ordinairement bicolore.

$A$ a Bec plus court que la tite, queue échancrée, à rectrices larges, poitrine à plumes colorées.

Aß Bec aussi long que la moitié du corps, queue fort échancrée, à rectrices larges, blanches à la base, poitrine blanche.

Adelomyia

Rhamphomigron

Oreonympha

Ah Bec assez large, fort comprimé dans le quart de sa longueur en une lame très mince, une touffe bleue violette se détachant de chaque côté du cou.

A a. Queue médiocre, arrondie.

$A \beta$ Queue longue, à rectrices externes courtes.

Heliothrix

B Oiseaux ornés d'une huppe, d'une collerette, ou à queue singulière, à narines courertes de plumes frontales, à base de l'arête du bec non visible.

$B a$ Une bande blanche ou roussâtre en travers du dos inférieur. 
BA Tête surmontée d'une huppe plus ou moins haute et large, une collerette de plumes allongées, queue normale arrondie.

Bb Rectrices externes atténuées et prolongées, tarse garni d'une manchette.

BB Oiseaux sans bande au dos.

Gouldia

Ba Queue à rectrices externes prolongées, dénuées, terminées par une palette.

Ba Rectrices externes réduites à la baguette seule ou à peu près et terminées par une palette plus ou moins dilatée, pattes à manchettes.

Steganura

$B \beta$ Rectrices externes prolongées fort courbées, réduites à la baguette seule et terminées par une palette fort dilatée, les autres rudimentaires ou nulles, les deux souscaudales postérieures fort prolongées et linéaires.

\section{LODDIGESIA}

Bb Queuc longue profondément fourchue divisée en deux branches, à rectrices parallèles fort étagées.

$B_{a}$ Tite couverte au sommet d'une plaque squamiforme fort luisante, rectrices bleues ou vertes.

Cynanthus

Bß Sommet de la tête non squamiforme.

$B 1$ Rectrices rouges ou dorées.

$B$ : Rectrices externes à barbe externe cen- . drée ou grise dans sa partie basale, assez étroites et faiblement métalliques.

LESBIA

C Queue ordinairement irrégulière, gorge et devant du cou parés d'une cravate formée de plumes squamiformes rouges ou violettes fort splendides. ('" Rectrices médianes trìs courtes, submédianes linéaires et prolongées.

Thaumastera

C" Bec arqué plus long que la moitié du corps, queue fort fourchue, à rectrices assez fines, l'externe presque parallèle dans toute sa longueur.

RHonopis

C"' Rectrices médianes courtes, les autres allongées, égales, parallèles, arrondies à l'extrémité et rigides.

Mrrtis

C"., Bec droit, ordinairement plus court que la moitié du corps, queue profondément fourchue, à rectrices assez larges, l'externe aiguë au bout. Calliphlox

("',"' Bec droit ou presque droit, moins long que la moitié du corpes, quelques-unes des rectrices linéaires trìs fines ou fort atténuées. 
C.A Queue très courte ne dépassant pas les tectrices, à trois rectrices latérales de chaque còté fort atténuées, rigides et plus courtes que les suivantes.

Mrritia

$C B$ Queue dépassant les tectrices.

Ca Deux rectrices latérales fines en lame de poignard, troisième large, graduellement atténuée vers l'extrémité, qui est aiguë, deuxième et troisième égales et les plus longues.

Acestrera

Cb Rectrice externe très mince, les deux suirantes fines subparalleles, la troisième plus longue.

Ch.etocercus

\section{GeNre GLA UCIS}

a Dessous roussâtre; queue roux cannelle traversée par une bande noire devant l'extrémité blanchâtre, rectrices médianes bronzées.

G. Hirsutus

$b$ Gorge noire; queue ocreuse, à rectrices médianes d'un vert noirâtre.

G. Cervinicauda

\section{GeNhe PIIAETHORNIS}

a Rectrices médianes prolongées en un appendice atténué, aussi long environ que leur partie large et ordinairement blanc.

a' Bec presque droit, dessous faure pàle. Ph. Botrcieri

a" Bec courbé.

a Dessous cendré plus ou moins foncé, lustré en jartie de vert, dos et base des rectrices vert bleuâtre.

Ph. Emilate

ab Dessous faure roussàtre.

a Gorge grise traversée d'une ligne médiane fauve, plumes du croupion frangées de roux.

Pif. superciliosls

a $\beta$ Gorge blanche bordée des deux côtés d'une moustache grise, sous-caudales rousses.

Ph. syrmatophorus

ac Dessous gris, plumes du croupion bordées de gris.

Рн. hispides

$b$ Rectrices médianes peu prolongées en un appendice atténué graduellement, à extrémité blanche ou rousse, queue du or arrondic.

$a^{\prime}$ Dessous roux. 
ba Rectrices médianes noirâtres devant l'extrémité.

Ph. griseigularis

$b b$ Gorge grise striée de noirâtre.

Ph. strmiglaris

b" Bande pectorale distincte chez le $0^{7}$, mandibule orangée en grande partie.

Ph. nigriginctus

\section{Gente APIIANTOCHIROA}

Dessus du corps vert.

a Dessous blanc tacheté de vert.

$b$ Plaque gulaire squamiforme d'un rouge lilas.

A. Hyposticta

A. gularis

\section{GENRE OREOTROCHILUS}

Plaque gulaire verte, squamiforme chez le ơ.

a Dessous du corps blanc avec une bande médiane noire.

O. Leucopleurus

$b$ Dessous du corps blanc avec une large bande médiane brun châtain.

O. Estella

$c$ Dessous du corps noir velouté lustré de bleu.

O. MELANOGaster

\section{GeNRE DORYFERA}

a Dessous du corps noir chez le ơ gris verdâtre chez la o, plaque frontale violette chez le ơ, vert bleuâtre chez la @ .

I). JOHANNA

$b$ Dessous du corps olive grisâtre, plaque frontale verte.

I). RECTIROSTRIS

\section{GenRe JOL EMA}

a Front sans éclat métallique, une tache violet améthyste squamiforme sur le devant du cou. J. Scirreibersi

$b$ Plumes frontales fort étincelantes, poitrine verte. J. whitelyana

\section{Genre ThaLURANIA}

Gorge et devant du cou largement vert brillant.

a Dessous du corps bleu de Prusse, taille plus forte.

T. NIGROFASGIATA

$b$ Dessous du corps bleu de Prusse, sommet de la tête cuirreux, taille plus faible.

T. JELSKII 


\section{Genre LOPIIORNis}

$a$ Gorge d'un vert métallique', huppe rousse ponctuée de noir.

L. Delattrii

$b$ Gorge non métallique, huppe verte ponctuée de blanc, dessous brun foncé, à lustre bronzé.

L. Verreauxi

\section{Gente G0ULdi}

a Huppée, à poitrine noire non métallique.

ú. Popelairi

$b$ Sans huppe, poitrine vert métallique bordée de rouge.

G. LANGSDORFFi

\section{GENRE MYRTIS}

$a$ Gorge d'un vert métallique bordée de violet, quatre rectrices latérales également larges dans toute leur longueur.

M. FANYY

$b$ Gorge d'un rouge violet métallique passant au bleu, trois rectrices latérales atténuées et pointues.

M. Yarrelli

\section{GenRe S'TEGANURA}

Plumage du tarse roux.

a Rectrice externe courbée en dedans, à palette subpentagonale à peine plus longue que large. S. PERtava

$b$ Rectrice externe droite, à barbe non barbée jusqu'à la palette.

$b$ 'Palette ovale allongé, dessous du corps vert. A. solstitials

$b$ " Palette subpentagonale, dessous blane tacheté de vert.

S. ADD.E

$c$ Rectrice externe droite toute barbéc, palette oblongue subarrondic au bout.

S. CISSIURA

\section{GENRE LESBIA}

a Rectrice externe noire lustrée trìs légèrement de vert, à tache terminale verte, le gris blanchâtre de la barbe externe dépassant longuement la rectrice submédiane.

I. Gracilis

$b$ Rectrice externe noire dans la moitic basale, puis verte terminée par une tache brillante vert olive tirant au rouge, le gris de la barbe externe n'atteignant pas l'extrémité de la rectrice submédiane. 


\section{Genre CYNANTHUS}

a Rectrice externe noir bleuâtre jusqu'à l'extrémité des subexternes, puis d'un vert très brillant, dessous d'un vert bronzé.

C. HocoA

$b$ Rectrice exterme noir indigo, plus bleue après avoir dépassé la submédiane, extrémité (?), dessous gris cendré, à plumes bordées de blanchâtre.

C. GRISEIVENTRIS

\section{Genre SAPlPIO}

$a$ Gorge vert métallique, queue tries brillante d'un rouge vineux ou cramoisi, à extrémité des rectrices noire.

S. PHAON

$b$ Gorge rouge, rectrices sans noir terminal.

S. CAFOLI

\section{Gexpe AGLEACTIS}

Dessous roux.

a Touffe pectorale faure.

$a$ ' Tout le dessous roux. A. Cuprempenns

“" Poitrine et abdomen d'un brun bronzé. A. Cauratonota

b Touffe pectorale blanche, croupion lilas pourpré. A. Casternavin

\section{GENRE RHIAHPIIOHICRON}

$a$ Dessus du corps d'un bleu violâtre, rabat gulaire vert doré large et unicolore.

R. MIGRORHYNGHUS

$b$ Rabat gulaire étroit bicolore.

b' Sommet de la tête concolore au dos.

$b^{\mathrm{a}}$ Couleur générale olive, rabat vert en liaut et d'un violet rougeâtre en bas; queue olive bronzée.

R. OLITACELS

$l^{\text {b }}$ Couleur du dessus blene, rabat vert en haut, violet en bas; queue bleue verdatre. R. Stanlest

$b$ " Sommet de la tête roux marron rougeâtre, queue bronzée.

R. RUFIGEPS

\section{Gevie HETALLURA}

a Rabat gulaire rouge, couleur générale bronzée, queue bronzée changeant au bleu.

M. Eupogon

b Rabat gulaire vert. 
$b$ Plumage général noir avec un faible lustre rougeattre.

$b^{\mathrm{a}}$ Queue rouge vincux changeant au bleu.

$b^{\text {b }}$ Queue cuivieuse.

M. JelskiI

M. OPACA

b" Vert en dessus.

$b^{c}$ Queue d'un pourpré bronzé, dessus du corps vert.

M. TYRLANTHN.

$l^{\text {d }}$ Queue violet changeant au bleu, dessus du corps vert.

M. silaragdinicollis

$l^{\circ}$ Queue bronzée changeant en bleu.

M. ENEIGAUDA

\section{Gente A DELOHYIA}

Dessous du corps isabelle sale à flanes rerts.

a Gorge maculée de bleu.

$b$ Gorge maculée de vert.

c Gorge maculée d'olive brunatre.

A. INohixate

A. chlonosphlat

A. MELANOGEYYS

\section{GeNRE PETASOPHORA}

a Plumage général vert métallique.

a' Touffe auriculaire rouge violàtre, sous-caudales blanches.

P. SEliRiRostris

$a$ "Touffe auriculaire d'un bleu foncé.

['. Anatis

a"' Touffe auriculaire d'un bleu violâtre.

P. cyanotis

$b$ Plumage général nou métallique, touffe auriculaire d'un bleu violet.

P. DELPHINE

\section{GeNRE HLLIANTHEA}

Dessous du corps revêtu de plumes roussâtres à la base.

a Queue roux pâle, à extrémité rert bronzé, prase jugulaire violette, tectrices alaires d'un vert cuivieux.

$b$ Queue fauvę roussâtre terminće de vert bronzé, prase jugulaire bleu saphir passant au violet, tectrices alaires d'un rouge cuirreux.

II. OSCUL.INS

II. DICHROLRA

\section{GLARE II ELIOTIR Y PII}

$a$ Gorge d'un violet très brillant.

II. VIOLA

$b$ Gorge d'un rouge feu très brillant.

II. MICRASTER 


\section{Gente DIPIILOG RNA}

a Plaque céphalique d'un vert très brillant passant aø bleu, avec une tache saphirée sur le milieu du cervix, dos cannelle.

D. WARSZEIVICZI

$b$ Plaque céphalique rouge feu très brillant passant au vert, avec une tache cervicale saphirée, dos bronzé noirâtre.

I). 1Ris

\section{Gente Bourcieria}

a Anneau collaire roux vif, interrompu au milieu du dos.

$b$ Bande pectorale blanc pur très large, front vert métallique très luisant.

B. INSECTIYORA

c Dessus cuivreux rougeâtre, dessous fuligineux, à gorge et devant du cou blancs maculés de fuligineux.

B. GOELIGENA

\section{Gente Floricola}

$a$ Sommet de la tête et cou vert métallique, souscaudales brunes bordées de blanc.

F. LONGIROSTRIS

$b$ Sommet de la tête et cou d'un vert métallique pâle, sous-caudales blanc grisâtre.

F. ALBICRISSA

\section{Genre EIRIOCNEMIS}

a Touffe tars le noire.

E. DERBIANA

$b$ 'Touffe tarsale d'un brun pâle.

E. AFFinis

c Touffe tarsale blanche.

$c$ 'Sous-caudales d'un saphir brillant, tout le dessus vert cuivreux, queue noire bleuâtre.

c" Sous-caudales vertes, à base fauve blanchâtre, dessous vert bleuâtre, à plumes du milieu de la poitrine blanches à la base, queue d'un vert bronzé.

E. Dybowskif

E. SAPphiropygla

\section{GeNRE LEUCIPPUS}

a Queue à rectrices externes blanches sur la barbe interne, gorge blanc pur. 
a' Vert doré du dessus du corps et des flancs brillant.

L. GHIONOGASTER

$a$ " Vert doré du dessus du corps et des flancs peu brillant.

L. PALLIDUS

b Queue verte, à extrémité des rectrices gris pâle, gorge maculée.

L. CHLOROCERCUS

$c$ Queue verte en entier, de petites macules d'un vert très brillant sur les còtés de la gorge et du coll.

L. VIRIDICAUD $\Lambda$

\section{GENRE IGYRTRIA}

a La gorge et le haut de la poitrine vert bleuâtre, queuc bleu d'acier.

A. Barthetit

$b$ Gorge et poitrine vert métallique, front et nuque vert sombre, queue noire.

A. Fluviatilis

\section{GeNRE AMAZILIA}

Poitrine blanche, abdomen et flancs roux, queue marron pâle.

$a$ Gorge d'un vert émeraude métallique. A. pristixa

$b$ Gorge d'un vert doré métallique. A. Leugoph.EA

\section{GENRE HYLOCIIARIS}

a Menton roux, poitrine bleu saphiré pâle, tìte verte.

H. SAPPHIRINA

b Menton blanchâtre, poitrine bleu violâtre, tète bleue.

H. GYANE.I

\section{GENRE CHLOROSTILBON}

Bec noir en entier.

a Queue courte subarrondie.

b Queue médiocrement longue, échancrée.

Ch. prasines

Ch. Melanorhrofats 


\section{Fanille CCEREBID A}

A Bec droit.

A' Bec plus long que la moitié de la tête.

AA Comprimé beaucoup plus élevé que large.

Aa Màchoire terminée d'un crochet plus ou moins courbé; extrémité de la mandibule arquée.

Diglossa

$A b$ Crochet maxillaire peu courbé, mandibule droite.

Diglossopis

$1 B$ Bec ron comprimé.

Aa Conique, à extrémité aiguë.

Conirostrun

1b Élargi à la base, à dos de la nıâchoire légèrement courbé à l'extrémité; deuxième ( Dacxis rémige la plus longue.

Ac Bec un peu plus long que la tête, à dos arqué depuis les narines; troisième et quatrième rémiges les plus longues.

A" Bec plus court que la moitie de la tête. Xenodacnis

Chlobophanes

B Bec plus ou moins arqué.

B' Coloration différente dans les deux sexes, cou-

leur bleue prédominante dans les mâles. Cofreba

B" Coloration semblable dans les deux sexes. Certhola

\section{Gevie DIGIOSSA}

a Plumage général noir.

$a^{\prime}$ D'un noir intense luisant en entier. D. Aterriata

a" Moustaches blanches, bande pectorale rousse

et blanche, sous-caudales rousses. D. PECTORALis

a"' Ardoisé foncé, à flancs de l'abdomen blancs. D. albilateralis

a"”, Dessus noir, dessous roux, à flancs, scapu-

laires et croupion cendrés.

D. BRUNNEIVENTRIS

$b$ Dessus cendré bleuâtre, dessous roux.

D. SITTOÏDES

c Plumage général bleu, à front, côtés de la tête et menton noirs.

D. personata

\section{GENRE CONIROSTRU I}

a Plumage général noir, sommet de la tête, épaules et croupion bleus.

C. ATROCTANEUM

$b$ Dessous du corps roux.

b' Dos bleu. 


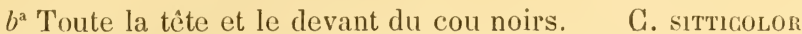

$1,^{\text {b }}$ Sommet de la tête noir, côtés de la tête et devant du cou schistacés.

C. Graneum

b" Dos schistacé, sornmet de la tête noir, sour-

cils roussâtres.

C. FERRUgineIVENTRE

c Dessus cendré grisattre, dessous isabelle grisàtre, sourcils et miroir alaire blancs.

C. Ginereuil

\section{GeNre DACNis}

a Plumage général bleu.

a' Dos, gorge ef lores noirs. D. CAYANa

a" Dos, front, côtés de la tête noir's, milieu du ventre et sous-caudales blancs.

D. ANGELIGA

b Tête et dos noirs, à demi-anneau nucal jaune, dessous straminé verdâtre, ailes bleues.

D. PULGHERmina

$c$ Corps jaune, à sommet de la tète olive verdâtre, gorge, milieu du dos, còtés de la tète, ailes et queue noirs.

D. FLAVIVENTER

$d$ Dessus cendré bleuâtre.

d' Dessous grisâtre pâle.

D. PLuMbeA

d" Dessous cendré bleuâtre, grosse raie postoculaire blanche, sous-caudales rousses.

D. ANALIS

\section{Gente CorreBi}

a Tout le corps bleu, à gorge et lores noirs.

$a$ ' Le noir de la gorge non prolongé sur la région jugulaire.

C. c.firulea

$a$ " Le noir prolongé jusqu'au milieu de la poitrine.

C. NITIDA

$b$ Bleu à dos et lores noirs, milieu de l'occiput bleu céladon.

C. GYANEA

\section{GeNre CERTHIOLA}

a Noir en dessus, à sommet de la tête à peine plus foncé que le dos, croupion jaune.

C. Luteoli

$b$ Dos schistacé, à sommet de la tète noir.

b' Miroir alaire blanc plus ou moins développé.

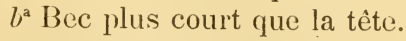

C. perUThana

$b^{b}$ Bec plus long que la tête.

G. magnirostris

b" Miroir alaire nul.

G. ChloropyGa 


\section{FaMILLE VIREONIDAE}

A Bec faible sylviforme.

A' Ailes aiguës, à deuxième rémige la plus longue. Vireosrluia

A" Ailes émoussées, à troisième et quatrième rémiges les plus longues.

Hylophilus

B Bec robuste laniiforme.

B' Bec presque aussi long depuis la commissure que la tète, peu élevé.

b' Bec court, élevé et fort comprimé.

Vireolanils

Crglories

\section{GENTE VIREOSYLVIA}

$a$ Sommet de la tête cendré.

a' Sourcil blanc bordé en dessụs de noir; flancs

du corps grisâtres très peu lavés de verdâtre. V. olivacea

$a$ " Sourcil blanchâtre sans bordure noire; còtés

du cou et de tout le corps largement jaunes. V. FLA ro-viridis

$b$ Sommet de la tète brun, sourcil blanc, abdomen jaune.

V. JoSEPHA

\section{GENRE IIYLOPHILUS}

a Front roussàtre, dessous du corps gris lavé d'olive.

H. FerRUgiNeIFroNs

$b$ Sommet de la tête gris brunâtre, gorge et cou antérieur gris blanchâtre, le reste du dessous jaune sale.

H. FLAVIVENTRIS

$c$ Olive verdâtre en dessus, jaune verdàtre en dessous.

H. OLIVAGELS

\section{GeNle CYCHORHIS}

a Sommet et côtés de la tête cendrés, à sourcils marron.

C. guilnensis

$b$ Sommet de la tète vert jaunâtre concolore au dos, sourcils marron foncé.

C. virentigeps

$c$ Tout le sommet de la tête marron foncé.

C. Contrerasi 


\section{FAMILLE SYLVIDE}

A Bec faible, sylviforme; taille petite.

Polioptila

B Bec court, déprimé; taille médiocre.

Myiadestes

\section{Genre PoLioptila}

a Sommet de la tète noir.

$a^{\prime}$ Lores blancs.

P. BILINEATA

a" Lores noirs.

aa Noir basal des rectrices externes non couvert par les tectrices; taille plus forte. P. Nigriceps

$a b$ Noir basal des rectrices externes couvert par les tectrices; taille plus faible.

P. PARViRostris

\section{GENRE MYIADESTES}

a Roux olivâtre en dessus, le front et tout le dessous cendré.

M. RALLOÏDES

$b$ Dos cannelle rougeâtre, dessous noir, grosse bande faciale blanche.

M. LELCOTIS

\section{FayilLe MOTACILLIDE}

\section{GeNre ANTHUS}

a Rectrice externe toute blanche; ongle plus long que le pouce.

A. correndera

$b$ Rectrice externe blanc sale presque en entier; ongle plus long que le pouce.

A. peruthanes

c Rectrice externe roussâtre dans sa moitié externe; ongle un peu plus long que le pouce.

A. Bogotexsis

d Rectrice externe blanche en grande partie; ongle égal au pouce.

A. Furcatus 


\section{FAMILLE MNIOTILTIDAE}

A Cils maxillaires petits.

A' Du blanc ou du jaunc à la queue.

AA Première rémige plus courte que la deuxième; coloration semblable dans les deux sexes.

PAILLA

$A B$ Première rémige égale à la deuxième et les plus longues; coloration différente dans les sexes.

DENDROEG.1

1" Queue unicolore.

A.1 Bec large à la base, non comprimé dans sa moitié terminale.

Mrionloctes

.1 li Bec peu large à la base, comprimé dans sa moitié terminale.

Geothlypis

B. Cils maxillaires fort développés.

I3' Queue unicolore.

Bisileuterus

b" Queue à rectrices externes blanches en grande partie.

Setophaga

\section{GENRE DENDIRACA}

a Barbe interne des rectrices blanche en grande partie.

a' Dessus du corps noir, dessous jaune varié de noir sur les còtés.

D. Blagkburni.e

a" Bleuâtre en dessus, blanche en dessous.

D. Gerule $A$

$b$ Barbe interne des rectrices jaune, dessous jaune tacheté de roux.

$b$ ' Sommet de la tête roux.

D. AUREOLA

$b$ " Sommet de la tête concolore au dos.

D. Astiva

\section{GeNre GEOTHLYPIS}

a Sommet de la tête cendré, front et lores noirs.

a' Le cendré occupant la région postoculaire, le noir couvrant presque en entier les tectrices auriculaires.

G. velata

a" Toute la région postoculaire et auriculaire verte, le noir fin au front et ne dépassant pas le bord postérieur de l'cil.

G. ilkicul.iris

a" Région postoculaire et auriculaire verte, le noir dépassant largement, mais très peu le bord postérieur de l'œil.

G. AQQuinoctialis, peRUVIANA 


\section{Gevre BasILEUTERUS}

a Quene bicolore, olive sombre en dessus, ocreus en dessous.

B. UROPYGIALIS

b) Queue unicolore.

b' Olive brunâtre en dessus, blanc et cendré en dessous, milieu du sommet de la tète d'un roux vif bordé des deux côtés d'une ligne noire.

B. Castaneiceps

$b$ " Olive verdâtre en dessus, jaune en dessous. $b^{\text {a }}$ Milieu du sommet de la tìte roux bordé des deux côtés d'une ligne blanche, gorge et còtés de la tête cendrés.

B. coronatus

$l^{\text {b }}$ Milieu du sommet de la tête roussâtre bordé des deux côtés d'une large raie olive noirâtre, gorge jaune, sourcils jaunâtres longs.

$b^{\text {c }}$ Milieu du sommet de la tête gris bordé des deux côtés d'une large raie noirâtre, sourcils gris pâle, gorge blanchâtre.

B. trifasclatus

$b^{\text {d }}$ Sommet de la tète noir, traversé de trois raies gris blanchâtre, gorge blanchâtre. B. Tristriatus

$b^{\text {e }}$ Sommet de la tète concolore au dos, à sourcils jaunes assez larges.

$b^{i}$ Vilieu du sommet de la tête noir.

13. LUTEOVIRIDIS

13. Nigiteristites

\section{GeNre SETOPIIAGA}

a Ardoisé en dessus, jaune en dessous.

$a^{\prime}$ Gorge jaune.

$a^{\text {a }}$ Sommet de la tête tout noir.

S. metanogephata

$a^{\mathrm{b}}$ Sommet de la tête noir avec une grosse tache occipitale fauve roussâtre.

S. Batrdi

$a$ " Gorge noire, une grande tache occipitale marron rougeâtre.

S. verticalis

$b$ Noir en dessus; queue roux orangé à la base.

S. RUtigilla 


\section{FAMILLE TURDIDE}

A Queue plus courte que l'aile.

A' Coloration semblable dans les deux sexes.

AA Tarse long et grêle, d'une couleur claire. Catriarus

$A B$ 'Tarse plus ou moins épais, à couleur foncée. Tundus

$A$ " Coloration plus ou moins différente dans les

sexes, les mâles noirs ou noirâtres.

Merula

B Queue plus longue que l'aile, fort arrondie; corps srelte.

Mrnes

\section{Genre CaTHARUS}

a Dessous du corps blanc au milieu, immaculé. C. Fuscater

$b$ Dessous du corps roussâtre maculé de noirâtre. C. DRYas

\section{GENRE TURIUS}

a Maculé en dessous.

$a^{\prime}$ De noirâtre jusqu'à la région anale.

T. mara ̃̈oneus

$a$ " De brun foncé jusqu'au haut de l'abdomen; tour de l'œeil roussâtre.

T. Swarsoni

$a$ "' De brun foncé jusqu'au haut de l'abdomen; rien de roussâtre autour de l'œil.

'T. Aricie:

$b$ Dessous du corps immaculé, excepté la gorge striée de noir ou de brun sur un fond blanc.

b' Dessus du corps cendré bleuâtre, dessous blanc, à flancs et sous-alaires ocreux.

T. ReEvi

b" Dessus du corps brun foussâtre, dessous cendré pâle, sous-alaires grises.

T. S.ATCLITUS

b" Dessus gris terreux foncé, dessous plus pâle, à milieu du ventre blanc, sous-alaires grises; stries gulaires fines.

T. tgxobilis

b"," Dessus brun tirant légèrement au cannelle, dessous gris soyeux, à milieu du ventre blanc; stries gulaires très grosses, sous-alaires gris roussâtre.

'T. GROTOPL'\%'

b",'” Dessus brun marron, dessous brun, à milieu du ventre blanchâtre, sous-alaires roux orangé vif.

T. HAUTWELLI

b",", Schistacé très foncé, à tête noire, dessous moins foncé, à milieu du ventre blanc, stries gulaires grosses, sous-alaires ardoisćes.

T. Nigriceps 


\section{Genke MERULA}

a Plumage général noir.

a' Queue fort arrondie à l'extrémité.

M. SERraNA

a" (Queue coupée carrément à l'extrémité.

M. LEUGOPS

$b$ Plumage général fuligineux.

b' Gorge immaculée.

M. GIGANTODES

b" Gorge striée de foncé.

M. chiguanco

\section{FaMILLE TROGLODYTID尼}

A Queue distinctement plus longue que l'aile, non rayée en travers.

Doxacobius

B Différence entre la longueur de la queue et de l'aile petite ou nulle.

P' Queue à peu près égale à l'aile.

BA Bec faible, aussi long ou plus long depuis la commissure que la tête; plumage fort tacheté et rayé.

B" Queue un peu plus longue ou un peu plus courte que l'aile; bec plus court que la tête; plumage du corps non rayé ni tacheté, des lignes transversales sur les rémiges et la queue. Cirveentma $D^{\prime \prime \prime}$ Queue un peu plus courte que l'aile.

BA Bec à échancrure subterminale.

Ba Narines ovalaires, ouvertes, situées près du bord antéricur de la fosse nasale.

ThrYopintes

Bb Narines longues et étroites, couvertes en dessus diune membrane.

T'ryothores

BP Bec sans échancrure subterminale.

$\mathrm{Ba}$ Bec depuis la commissure égal ou plus long que la tête; dos unicolore ou rayé en travers de foncé d'une manière peu distincte.

Troglodvtes

Bb Bec moins long que la tête, une grosse tache noire striée de blanchâtre au dos. Cistotinorus

G Queue beancoup plus courte que l'aile.

C' Bec beaucoup plus court que le tarse.

$C A$ Bec faible, peu élevé à la base, à narines situées dans la partie antérieure de la fosse nasale.

Cl Bec fort, élevé ì la base et fort comprimé 
entre les narines; ces dernieres ouvertes dans le milieu de la fosse nasale.

Ciphorhinus

C'” Bec un peu plus court que le tarse, à peu près aussi haut que large vis-à-vis les narines; les narines ouvertes au milieu de la fosse nasale sont couvertes en dessus par une membrane en les atténuant sur le devant.

Migrocergulus

\section{GeNRE CAMPYLORIIYCIIUS}

a Bande sourcilière large, dessous blanchâtre varié de brun.

$a$ ' Taches de la poitrine subarrondies et isolées. C. Balteatus

$a$ " Taches de la poitrine transversales, réunies en bandes continues.

C. Fasciatus

$b$ Bande sourcilière fine, dessous du corps faure varié de brun.

C. hypostictus

\section{GeNRe CYPHORHINUS}

$a$ Tout le sommet de la tête schistacé olivâtre, devant et còtés du cou largement et la poitrine roux.

C. THoracicts

$b$ Sommet de la tête roux.

$b$ ' Taille plus petite. C. Montrator

$b$ " Taille plus forte, couleur dı dessus un peu plus roussâtre.

C. Salini

\section{Gente NiCROCERCULUS}

a Dessous du corps blanc pur.

M. BHGOLOR

$b$ Dessous du corps blanc squamulé de brun.

M. MARGINATUS

\section{GENRE HENICORIIINA}

$a$ Poitrine et devant de l'abdomen cendré passant un peu au blanchâtre sur le milieu même de ce dernier.

II. LEUCOPHRYS

b Gorge, devant du cou, poitrine et milieu de l'abdomen blanes.

II. LELCOSTICTA

\section{GeNRE TIIRYOPIILUS}

a Roux en dessus, à sourcils et le dessous dụ corps blancs soyeux, région anale ocreux roussâtre. 
$b$ Brun roussâtre en dessus; sourcils, gorge et haut de la poitrine blancs, abdomen roux.

c Brun roussatre en dessus; sourcils fauve griSâtre, dessous brun terreux pàle.

Th. Fulves

\section{GenRe THIRYTHIORUS}

a Dessous du corps maculé.

$a$ ' Des raies noirâtres irrégulières sur un fond blanc sur tout le dessous du corps.

Th. Sclateri

$b$ Dessous du corps immaculé, région auriculaire noire.

b' Région auriculaire striée de blanc.

$b^{2}$ Poitrine et milicu de l'abdomen cendrés, queue rayée de noir et de gris ou roussâtre.

TH. GRISEIPEGTUS

$b^{\text {b }}$ Gorge, milieu de la poitrine et plus largement le milieu de l'abdomen blancs, queuc rayée de gris et de noir.

TH. ALBIVETRIS

- b" Région auriculaire sans stries, poitrine et abdomen gris au milieu, queue rayée de roux et de noir.

Th. cantator

\section{GeNRE TROGLODYTES}

a Sourcil large et nettement prononcé roussâtre pâle.

T. SOLSTITIALIS

$b$ Sourcil nul ou à peine indiqué.

b' Brun grisâtre en dessus, à dos rayé de noirâtre d'une manière plus ou moins prononcée, gorge isabelle.

T. TESSEllatus

b" Brun en dessus, rayé au dos de noirâtre, gorge blanche.

T. RUfults

$b$ "' Brun terreux en dessus sans raies foncées, tout le dessous fauve.

T. AUDAX 


\section{FamilLe PTEROPTOCHIDÆ}

\section{GENRE SCYTALOPUS}

$a$ D'un schistacé noirâtre unicolore.

S. magellanigus

$b$ Région anale et flancs du bas-ventre roux rayé de noir, la tête et tout le dessous schistacé soyeux, croupion roussàtre rayé de noir.

S. ACUTIRostris

c Semblable au précédent, d'une taille plus forte, derrière même du croupion légèrement roussâtre avec quelques raies noirâtres.

d. Milieu du ventre blanc, derrière du croupion peu roux rayé de noirâtre.

S. SYLVESTRIS

\section{FAMILLE FORMICARIID F}

\section{TRIBU THAMNOPHILIN $A$}

A Bec épais, non comprimé, plus large que haut à la base.

Cymbilanies

B Bec comprimé.

B' Région postoculaire largement dénuée.

Mrriel istes

$B$ "' Toute la tête emplumée ou peu dénuée derrière l'œil.

$B A$ Cils mandibulaires peu développés.

Ba Bec élevé à la base, narines ovalaires. Thumophilus

$B b$ Bec non élevé à la base, narines presque rondes.

Thaministes

$B c$ Bec plus ou moins plus faible, que chez le Thamnophilus.

Drsithamina

Bd Bec élargi à la base, narines rondes plus voisines du sommet, ongles courts.

Pygoptila

$B B$ Cils mandibulaires fort dévelopjés, bec

élargi à la base; narines rondes.

Thamnomanes 


\section{TRIBU FORMICIVORIN $A$}

A Bec mince, plus long que la tête.

Rhamphoc anus

B Bec plus court ou aussi long que la tête.

B' Tecture du devant du tarse divisée en scutelles.

BA Queue courte, taille petite.

Myrmotherula

$B B$ Queue presque aussi longue ou plus longue que l'aile.

$B a$ Queue fort étagée.

$B_{\alpha}$ Bec graduellement atténué sans être comprimé.

$B 1$ Queue à douze rectrices.

Herpsilochues $B 2$ Queue à dix rectrices.

Cercomacra

B $\beta$ Bec comprimé dans sa moitié terminale.

$B 1$ Queue en partie blanche, taille faible.

Formicivora

$B 2$ Queue sans blanc, taille médiocre, mâle tout noir.

Prriglena

Bb Queue médiocrement étagée.

$B \propto$ Bec fort, faiblement comprimé dans sa partie terminale, taille médiocre. Percnostola

$B \beta$ Bec faible, comprimé dans sa moitié terminale, taille petite.

Terenura

B" Tecture du tarse glabre non divisée en scutelles, ou à scutelles à peine distinctes.

$B A$ Rémiges quatrième, cinquième et sixième égales et les plus longues.

$B a$ Bec grêle, queue étagée.

Myrueciza

$B b$ Bec large à la base et peu comprimé, plus court que la tête, queue coupée carrément ou arrondie.

Hypognemis

$B B$ Rémiges quatrième et cinquième les plus longues, bec un peu plus long que la tête.

Heterochenis

\section{TRIBU FORMICARIIN $A$}

A Tour de l'œil dénué; plumules nasales érigées.

Phlogopsis

B Tête tout emplumée.

B' Tarse très élevé, queue courte, quatrième et cinquième rémiges les plus longues.

BA Narines rondes, bec comprimé à l'extrémité. Grallaria

$B B$ Narines oblongues, bec non comprimé à

l'extrémité.

Chan eza

b' Tarse médiocre, queue assez courte. 
$B A$ Quatrième et cinquième rémiges les plus longues, scutelles du devant du tarse fort prononcées.

Formicarius

$B B$ Quatrième rémige la plus longue, devant du tarse glabre ou à scutelles peu distinctes.

Pithys

\section{TRIBU CONOPOPHAGIN $\mathrm{E}$}

A Bec court, assez large, non comprimé; narines rondes; rémiges quatrième, cinquième et sixième les plus longues.

Conopophaga

B Bec assez fin, presque aussi long que la tête; narines ovoïdes; quatrième rémige la plus longue.

Conythopis

\section{GENRE 'THAMNOPIILUS}

$a$ Noir, à ailes brunàtres, partout finement ondulé de blanc.

Th. undulates

$b$ Noir unicolore en dessus, blanc pur en dessous.

b' Queue noire sans taches blanches, sous-caudales blanches.

Th. melanurus

b" Queue noire, à rectrices externes maculées de blanc le long du bord externe, sous-caudales noires bordées à l'extrémité de blanc.

Th. transandeanus

$c$ Noir intense en entier.

c' Une grande tache humérale blanche.

$c$ "Toutes les tectrices alaires bordées à l'extré-

TH. Leuconotus mité de blanc, rectrices excepté les quatre médianes terminées d'une tache blanche, l'externe a aussi une tache blanche dans les deux tiers de la barbe externe.

Th. subandinus

c"' Plus fort que le précédent, à bordures blanches plus grosses aux tectrices alaires, couvrant le noir sur le devant de l'aile, point de tache blanche sur la barbe externe de la rectrice externe.

Th. subandinus Major

$c^{\prime \prime \prime \prime}$ Le pli de l'aile et les bordures des tectrices alaires blancs, le ventre ondulé de cendré clair.

Th. melanghrous

d Plumage général du corps cendré.

d' Sommet de la tète noir, des bordures blanches aux tectrices alaires et une tache terminale blanche aux rectrices.

$d^{a}$ Des taches noires au milien du dos, tout le dessous cendré.

TH. N.eVIUS 
$d^{\mathrm{b}}$ Des taches noires au milieu du dos, milieu de l'abdomen largement blanc.

Th. ALBIVENTRIS

$d^{c}$ Des taches noires au milieu du dos, souscaudales noires bordées de blanc.

Th. ayazonicus

d" Sommet de la tête noir, tectrices alaires sans bordures blanches, queue schistacée noirâtre.

Th. gapitalis

d" Sommet de la tête cendré, des bordures blanches très fines aux grandes et moyennes tectrices alaires et à l'extrémité des rectrices.

$e$ Capuchon noir couvrant toute la tête, la gorge et le devant du cou, bordé tout autour de blanc.

$e^{\prime} \quad$ Dos brun légèrement olivâtre, le noir du capuchon ne dépassant pas la poitrine.

Th. albinughalis

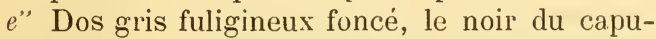
chon prolongé largement sur le haut de l'abdomen.

Th. LORETOYAGENSIS

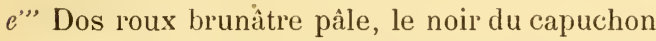
prolongé sur le milieu de la poitrine en une ligne peu large.

$f$ Tète et cou noirs, dos et queue d'un roux cannelle vif; dessous du corps noir rayé en travers de blanc.

Th. murinus.

Th. Leugauchen

Th. palliatus

$g$ Dos cendré, sommet de la tête et ailes roux à l'extérieur, dessous blanchâtre, à poitrine rayée en traver's de noirâtre.

Th. subfasciatus

$h$ Tout le plumage noir rayé en travers de blanc.

$h$ ' Plumes de la huppe longuement blanches à la base.

h" Plumes du sommet de la tête maculées de petites taches blanches, couleur noire prédominante sur la gorge.

Th. tenulfasgiatus

$h$ "' Plumes de la huppe noires en entier.

$h^{\mathrm{a}}$ Le blanc prédominant sur la gorge, raies noires moins larges que les blanches sur l'abdomen.

$h^{\mathrm{b}}$ Le noir prédominant sur la gorge, raies blanches beaucoup plus fines que les noires sur l'abdomen.

Th. Doliatus

Th. radiatus

Th. Berlepschi

\section{GENRE MYRMELASTES}

a Plumage général noir, avec une tache blanche sur le devant de l'aile voisine de l'avant-bras.

$b$ Plumage général plombé, plus foncé en dessus qu'en dessous, petite tache blanche à l'extrémité de toutes les tectrices alaires.

M. NigerRinus

M. plumbeus 


\section{Gente PYgOPIILA}

a Sommet de la tête noir, tectrices alaires terminées par une petite tache blanche subtriangulaire.

P. maculipennis

$b$ Sommet de la tête concolore au dos, tectrices alaires terminées par une grosse goutte blanche. P. IARgaritati

\section{GENRE DYSITHAMNUS}

$a$ Plumage général ardoisé.

$a^{\prime}$ D'un cendré beaucoup plus clair en dessous, blanchâtre au milieu du ventre, plumes du milieu du sommet de la tête noires bordées de cendré.

D. Schistaceus

a" Plumage de tout le corps d'un schistacé très foncé, tectrices alaires terminées par une tache blanchâtre très petite.

D. ARDESIACUS

b Devant de l'oiseau ardoisé, derrière du corps olive.

b' Gorge largement blanchâtre, le cendré du dessous dépassant peu la poitrine, tectrices alaires bordées ou terminées d'une petite tache blanche.

D. SEMIGINEREUS

b" Gorge cendrée comme la poitrine, le cendré du dessous prolongé sur l'abdomen, les grandes èt les moyennes tectrices alaires bordées très finement de blanc, les petites sans tache terminale.

D. TAMBILLANUS

\section{GENRE HERPSILOCHUUS}

a Une large bande sourcilière blanche.

$a$ ' Sommet de la tête noir unicolore, barbe externe des rémiges rousse, dessous du corps d'un jaune très pâle.

H. RUFIMARGinatus

a" Sommet de la tête noir tacheté au milieu de blanc, barbe externe des rémiges grise, dessous du corps blanc.

H, MOTACILLOÏDES

$b$ Sourcils composés de taches blanches isolées, sommet de la tête noir parsemé de macules blanches, bord externe des rémiges olive clair, dessous du corps jaune soufre.

H. AXILLARIS 


\section{GeNre MYRMOTHERULA}

$a$ Dessus du corps noir strié de blanc.

a' Dessous du corps jaune sulfureux, à gorge blanche.

M. PYGMEA

$a$ " Dessous du corps blanc strié de noir sur la poitrine.

$a^{\mathrm{a}}$ Plumes dorsales blanches à la base.

$a^{b}$ Plumes dorsales sans rien de blanc à la base.

M. Surinamensis

M. MULTOSTRIATA

b Flancs de l'abdomen d'un blanc éclatant.

b' Parties supérieures du corps d'un cendré plombé.

M. AXILLARIS

b" Parties supérieures du corps d'un noir intense. M. MEL ENA

$c$ Gorge, cou antérieur et milieu de la poitrine noirs, dos ardoisé foncé, tectrices alaires terminées d'une bordure blanche.

M. Menetriesi

$d$ Gorge noire tachetée de blanc.

d' Dos d'un roux rougeâtre plus ou moins intense.

M. HEMATONOTA

d" Dos brun terreux.

M. GULARIS

$e$ Gorge noire, plumage général cendré plombé, tectrices alaires noires terminées d'une tache blanche.

M. atrogularis

$f$ Gorge cendrẻe, plumage général cendré plombé.

$f^{\prime}$ 'Tectrices alaires noires, les grandes et les moyennes terminées par une bordure blanche formant deux lignes continues en travers de l'aile, les rémiges tertiaires et les secondaires terminées par une tache blanche, sousalaires cendrées.

\section{Hauxwelli}

$f^{\prime \prime}$ Tectrices alaires noires dans leur partie terminale, bordées de blanc, rémiges sans tache terminale blanche, sous-alaires blanches.

M. GINEREIVENTRIS

\section{GeNRE FOR MICIVORA}

a Dos brun roussâtre, dessous du corps et côtés de la tète noirs; cette dernière couleur bordée d'une raie blanche depuis la naissance du bec; jusqu'à la queue.

F. rufatra

$b$ Plumage du corps noir.

b' Plumes du dos longuement blanches à la base. F. BICOLOR

b" Plumes du dos longuement blanches à la base, celles du dos inférieur et du croupion toutes blanches. 


\section{Genre CERCOUACRA}

a Queue unicolore.

C. TYRANnina

$b$ Rectrices terminées par une grosse tacheblanche. C. cinerascens

\section{Genre Pyriglena}

$a$ Plumage général noir uniforme.

P. PICEA

$b$ Plumage général noir tirant au schistacé en dessous, tectrices bordées d'une ligne blanche assez fine.

P. SERYA

\section{Gente Perchostola}

$a$ Plumage général ardoisé, plus clair en dessous, sommet de la tête et gorge noirs, tectrices alaires noires, à bordure terminale blanche fine.

P. Funebris

$b$ Plumage général ardoisé foncé, le sonımet de la tête, la gorge, le devant du cou et le haut de la poitrine noirs, pli de l'aile blanc.

P. Fortis

\section{GeNRE IYRMTCIZA}

a Dos roux brunàtre, tête schistacéce, dessous jusqu'à la poitrine noir, ventre blanc.

M. HEMINEL ENA

b D'un fuligineux foncé, à milieu du dessous noir. M. maynana

\section{Gente IIYPOCNEMIS}

$a$ Sommet de la tête et devant du dos variẻs de blanc sur un fond noir.

a' Dessous blanc, flancs roux, poitrine variée de noir.

$a$ " Dessous jaune pâle.

H. cantator, peruVIANUS

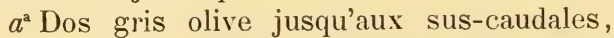
varié sur le devant de noir et de blanchâtre, flancs du ventre d'un roux pâle, queue olive brunâtre.

H. SUBFLAVA

$a^{\mathrm{b}}$ Dos gris olivâtre passant au roux brunâtre sur le dos inférieur et le croupion, varié sur le devant de noir et de blanc, flancs roux intense. queue brun roussâtre.

H. FLAVESCENS

$b$ Tète sans taches ni sourcils, dos tacheté. 
b' Plumage général cendré ardoisé, dos varié de grosses taches noires, avec une bordure blanche squamiforme, tectrices alaires noires également squamulées.

H. LEPIDONOTA

b" Dos noir maculé de fauve, dessous blanc, à gorge noire et une large bande pectorale composée de grosses macules noires.

H. Theresæ

b'" Milieu du dos noir parsemé de gouttelettes blanches, bande pectorale comme chez le précédent, gorge blanche bordée d'une grosse moustache noire atteignant les tectrices auriculaires.

H. NAVIA

c. Couleur générale ardoisée.

$c^{\cdot}$ Front d'un cendré très clair, gorge et côtés de la tête noirs.

$c^{\text {a }}$ Dessous du corps très clair, le cendré frontal prolongé en un sourcil très long, tectrices alaires noires squamulées de blanc. H. учгтонеrina

$c^{\mathrm{b}}$ Dessous du corps presque aussi foncé que le dos, le noir des joues n'atteignant pas le bord supérieur de l'œil, bande sourcilière très large.

H. LEUCOPHRYS

$c^{c}$ Milieu de la poitrine et de l'abdomen d'un cendré très clair, le noir dépassant le bord supérieur de l'œil.

H. LUGUBRIS

c" La tète et tout le cou noirs, tectrices alaires finement squamulées de blanc.

H. MELAJURA

c"' Milieu mème de la gorge couvert de plumes noires bordées de schistacé.

c"'" Gorge sans noir, un point blanc sur l'extrémité des tectrices alaires.

$d$ Tout le dessus du corps, le haut des côtés de la tète, les ailes et la queue noir intense, dessous blanc soyeux, taches blanches subtriangulaires sur les tectrices alaires.

H. MELANOPOGON

H. SGHistacea

H. HEMILELCA

\section{Gexre PITHYS}

a Huppe bifide et barbe blanches, dessus du corps schistacé, dessous et queue roux; point de ligne blanche derrière l'œil.

P. peruviands

$b$ Tout le dessus, les ailes et la queue d'un roux brunâtre; gorge, cou antérieur, milieu de la poitrine blancs, bordés largement de noir.

P. Ledcaspis

c Brun terreux à plumes du dos, les tectrices alaires, les rémiges secondaires et tertiaires ornées d'une tache antéapicale noire bordée en arrière d'une lunule ocreuse.

P. Lunulata 


\section{Gente FORMICARIUS}

a Front noir, nuque rousse, gorge et côtés de la tète noirs.

F. NIGRIFRONS

$b$ 'Tout le sommet de la tête brun olivâtre, gorge et devant du cou noirs, sous-caudales rousses.

I'. ANALIS

\section{Genre PHIOG0PSis}

a Dos et tectrices alaires à gouttes noires bordées de plus clair que le fond.

Ph. nigromagulata

$b$ Dos et petites tectrices squamulées de blanc.

Ph. ERYTHROptera

\section{GeNre: CHA I HZM}

a D'un olive roussâtre en dessus, le plus roussâtre sur la nuque et au croupion, tout le dessous blanc squamulé de noir.

C. NOBHLIS

$b$ Olive foncé en dessus, la gorge, le cou antérieur et la poitrine d'un ocreux vif, le reste du dessous blanc roussâtre squamulé de noir.
C. oldvacea

\section{Genke gratharia}

$a$ 'Toutes les parties supérieures du corps striées de blanchâtre, tout le dessous squamulé de noir. G. Andıola

$b$ Tout le dessus du corps distinctement squamulé.

b' Sommet de la tête ardoisé, dos brun olivâtre, le tout squamulé de noir; gorge brun foncé strié de roussâtre, dessous roux.

G. Regulus

b" Tout le dessus ardoisé foncé, squamulé de plus foncé; gorge blanchàtre bordće des deux côtés d'une moustache brune, dessous roussâtre squamulé de noirâtre.

G. squamigera

$c$ Dessus sans taches.

c' Dessus brun roussâtre, à sommet de la tête brun foncé, flancs roux immaculés.

G. Przewalsini

c" Dessus roux foncé, à sommet de la tête plus roux, la poitrine et les flancs de l'abdomen ferrugineux, à plumes bordées de blanc.

G. erythroleuca

c"' Roux brunâtre pâle en dessus, plus pàle en deșsous; taille petite.

G. RUFULA 
c"', Dessus olive grisâtre, à sommet de la tête roux brunâtre, lores et tour des yeux blanc isabelle, flancs variés largement de lignes noires et d'olivâtre.

G. ALBILORIS

$c^{\prime \prime \prime, ' ~ D e s s u s ~ o l i v e ~ r o u s s a ̂ t r e, ~ d e s s o u s ~ b l a n c ~ v a r i e ́ ~}$ fortement d'olive sur la poitrine et de cendré sur les flancs de l'abdomen.

G. MINOR

\section{Gente CONOPOPHach}

Fascicules postoculaires blanc soyeux.

a Sommet de la tête roux.

a' Dos brun olive, tout le dessous schistacé foncé.

G. castaneiceps

$a$ " Sommet de la tête et dos brun roussâtre, dessous cendré, à milieu du ventre blanc. C. TORRIDA

a"' Sommet de la tête brun roussâtre foncé, dessous gris cendré, à milieu de l'abdomen blanc, tectrices alaires terminées par une goutte fauve entourée d'une bordure noire.

C. peruviana

\section{FaMILLE DENDROCOLAPTIDE}

A Queue normale, à rectrices non raides.

$A^{\prime}$ Bec plus ou moins courbé.

$A A$ Queue dépassant peu le bout de l'aile, coupée carrément ou échancrée; ailes longues, à deuxième rémige la plus longue; bec parfois droit.

$A B$ Queue médiocre dépassant les ailes jusque Geositta près de la moitié de sa longueur, à première rectrice plus courte que les autres qui sont égales; ailes médiocres, à troisième rémige la plus longue; tarse élevé ; couleur rousse. Furnaruus

AC. Queue fort arrondie, dépassant les ailes dans sa plus grande moitié; ailes courtes, à troisième rémige la plus longue; bec grêle arqué dans toute sa longueur.

$A^{\prime \prime}$ Bec très peu arqué.

Upucerthia

$A A$ Queue courte, large, fort arrondie.

Aa Quatrième rémige la plus longue.

Lochmias

$A b$ Troisième rémige la plus longue, quatrième et cinquième presque égales. 
$A B$ Queue médiocre fort arrondie, à deux ou trois rectrices latérales terminées de blanc ou de gris; deuxième et troisième rémiges égales et les plus longues.

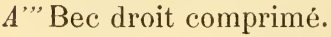

$A A$ Mâchoire terminée en crochet fort courbé. Ancisthrops

$A B$ Màchoire courbée légèrement au bout.

A $a$ Bec élargi à la base, à dos assez large et arrondi dans sa partie basale, à crochet terminal assez courbé; queue fort étagée.

Thripadegtes

$A b$ Bec à dos comprimé entre les narines, à crochet terminal à peine distinct.

$A \propto$ Queue étagée jusqu'à la troisième rectrice latérale inclusivement.

Cingledodes

$A \beta$ Première rèctrice plus courte, les autres presque égales.

Automolus

Philydor

A $\gamma$ Mandibule à barbe courbée en commençant à l'enfourchure.

Anabazenops

Ac Dos de la mâchoire droit jusqu'au bout, mandibule courbée en haut dans sa moitié terminale; queue assez courte, à rectrices latérales moins longues que les autres; du noir sur la barbe interne des rectrices intermédiaires.

Xenops

$A C$ Bec faible, peu comprimé, pointu.

Aa Queue fort ètagée.

A Queue longue à rectrices plus ou moins aiguës au bout.

A 1 Ailes courtes, à quatrième ou cinquième rémige la plus longue.

Synallaxis

$A 2$ Ailes longues et aiguës, à troisième rémige la plus longue.

Leptasthenura

$A \beta$ Queue longue, à rectrices larges et arrondies au bout.

Placellodonus

Ay Queue médiocre, à rectrices aiguës, couverte par les ailes jusqu'à la moitié. Phlokocryptes

$A \delta$ Queue médiocre; une touffe de plumes soyeuses au-dessous des oreilles se détachant du corps.

Pseudocolaptes

B Queue à rectrices raides, la baguette forte, dépassant les barbes en formant une pointe aiguë.

$B$ ' 'Taille petite.

$B A$ Bec fin terminé en pointe courbée légèreinent en bas.

Ba Bec médiocre; plumage non tacheté. Sitrasonus

Bb Bec court; plumage tacheté en dessous. Margakornis 
BB Bec à extrémité arrondie, aplatie, redressée un peu en haut.

Glyphorhyncilus

$B$ " Taille médiocre ou forte.

$B A$ Bec à peu près aussi long que la tête.

$B a$ Bec droit.

$B \propto$ Bec subtriangulaire atténué graduellement vers l'extrémité, non comprimé entre les narines, à dos faiblement arqué depuis la moitié de la longueur; plumage non maculé.

Dendrocincla

$B \beta$ Bec large, graduellement atténué vers l'extrémité, non comprimé entre les narines, à dos faiblement arqué depuis la naissance et arrondi; poitrine et cou maculés.

Dendroxetastes

By Bec fort comprimé dans sa plus grande moitié terminale et fort comprimé entre les narines, à dos légèrement arqué dans le tiers terminal; la tête, le cou et le dessous maculés. Dendrornis

$B b$ Bec légèrement arqué.

$B \propto$ Bec large à la base, élevé, comprimé en commençant en avant des narines. Xiphocolaptes

$B \beta$ Bec mince, comprimé en commençant en avant des narines.

Pigolaptes

$B \gamma$ Bec large atténué graduellement ver's l'extrémité, fort comprimé entre les narines.

Dendrocolaptes

$B B$ Bec dépassant le double de la longueur de la tête.

$\mathrm{Ba}$ Bec très faiblement fléchi. NASIGA

$B b$ Bec fort courbé dans toute sa longueur. Xiphocolaptes

\section{Genre GEOSI'TTA}

$a$ Bec droit, plus court que la tête, croupion isabelle, rémiges sans rien de roux.

G. SAXIGOLINA

$b$ Bec plus ou moins courbé.

$b$ ' Rémiges et rectrices sans rien de roux intérieurement.

G. MARITINA

b" Rémiges rousses en grande partie.

$b$ "' Bec long.

$b^{a}$ Bec grèle.

G. tenuirostris

$b^{\mathrm{b}}$ Bec épais.

G. CRASSIROSTRIS

$b$ "." Bec de la longueur de la tête ou plus court. $b^{\mathrm{c}}$ Première rectrice d'un roux isabelle, à 
barbe externe blanche, poitrine faiblement tachetée.

G. Juninexsis

$b^{\mathrm{d}}$ Première rectrice blanche jusqu'à la tache foncée terminale.

('. Frobeni

$b^{\text {e }}$ Première rectrice gris roussâtre à la base. Ġ. peruviana

\section{Genre FURNARIUS}

a Sommet de la tête terreux brunâtre.

$a$ ' Taille forte, parties supérieures du corps d'un roux cannelle vif, dessous du corps ocreux clair.

F. cinnamodeus

a" Taille petite, parties supérieures du corps d'un roux obscur.

F. MINOR

$b$ Sommet de la tête brun terreux.

$a$ "' Taille moyenne, parties supérieures du corps d'un roux plus clair sur le devant du dos, poitrine et abdomen d'un roux ocreux.

F. LeUCOPUS

c Sommet de la tête gris brunâtre foncé, taille moyenne, parties supérieures du corps d'un roux rougeâtre vif.

F. TORRIDUs

\section{Genre UPUCER'TII A}

$a$ Dessous du corps fort strié.

U. SERRANA

$b$ Dessous du corps non strié.

b' Poitrine faiblement nébulèe, queue brun roussâtre.

U. JELSKH

b" Poitrine unicolore, queue rousse.

U. PALLIDA

5*

\section{Genre CINCLODES}

a Tout le dessous du corps blanc pur.

C. palitatus

$b$ Dessous du corps fuligineux foncé strié de blanc. C. nigrofumosus

$c$ I)essous du corps blanc, à flancs gris, gorge et cou antérieur à ondulation grise incomplète.

G. rivularis

$d$ Dessous du corps gris roussâtre strié de blanc, gorge et cou antérieur blanc pur.

C. montanus

$e$ Dessous du corps gris strié finement de blanc, gorge blanche faiblement ondulée de gris foncé. C. BIFasciatus

\section{Genre LeP'TASTHENURA}

a Sommet de la tête roux brunâtre unicolore.

L. PILEATA

$b$ Sommet de la tête strié de noir.

$b$ ' Rectrices externes bordées de blanc. 
$b^{a}$ Dessous du corps d'un fuligineux strié de blanc.

I. ANDICOLA

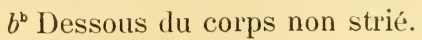

L. EGTTHALOIDES

b" Rectrices externos bordées de gris brunâtre clair.

L. Striata

\section{Genre SCLERURUS}

a Brun roussâtre, à croupion ferrugineux vif.

$b$ Olive foncé, à croupion concolore.

S. caudacutus

S. olivascens

c Brun roussâtre, à croupion marron rougeâtre, le cou antérieur et le haut de la poitrine roux.

\section{Genre SYMALLAXIS}

$A$ Queue à dix rectrices.

$a$ Sommet de la tête, ailes et queue roux.

a' Front ardoisé.

$a^{\mathrm{a}}$ Sourcil ardoisé.

S. FRONTALIS

$a^{\mathrm{b}}$ Sourcil fauve.

S. FRUTICICOLA

$a$ " Front roux, queue roux brunâtre.

S. Brunneicauda

$b$ Sommet de la tête et ailes roux, queue brune, front ardoisé, gorge et milieu du ventre largement blancs.

S. ALBESGENS

$c$ Sommet de la tête gris, ailes et queue rousses.

c' Gris olivâtre en dessus, plumes de la gorge schistacées, à bordures terminales blanches, poitrine grise.

c" Brun roussâtre au dos, la tète et tout le dessous cendré ardoisé.

S. PROPINQUA

" Gris roussâtre en dessus, gorge largement blanche, poitrine striée de brun.

S. MARAÑONIGA

S. Stictothorax

$d$ Tête et dos ardoisés, devant de l'aile roux, queue ardoisé brunâtre, gorge ardoisé noirâtre soyeux, à plumes du menton bordées de blanc.

S. TITHYS

$e$ Tout le dessus roux ou marron unicolore.

e' Rectrices fort atténuées dans leur partie terminale, gorge blanche sans jaune au menton. S. Mustelina

$e$ " Dessous du corps gris avec des taches grises au milieu de la poitrine, et fauves sur les côtés de la poitrine et le milieu de l'abdomen. S. vulpiña

$e$ "' Dessus de la tête et du corps brun marron, queue longue, à barbes fort désunies, anneau blanc autour de l'œil, tache rousse au menton.

$f$ Bande pectorale noire, sommet de la tête noir olivâtre, à sourcils blancs très longs et larges, 
dos olive grisâtre sans blanc à la base des plumes, poitrine et abdomen roussâtres.

S. paugalensis

$g$ Queue noire courte, bec robuste; rousse, à gorge noire.

$B$ Queue à douze rectrices.

$a$ Sommet de la tête, ailes et queue roux.

a' Dessous du corps non maculé.

$a^{\mathrm{a}}$ Sourcils blancs nettement prononcés, dos gris terreux, tout le dessous clair.

S. ANTISIENSIS

$a^{\mathrm{b}}$ Sourcils blanchâtres, dos gris olivâtre, tout le dessous gris foncé, à gorge blanchâtre.

S. SUBANDINA

$a^{\mathrm{c}}$ Sourcils ocreux, tout le dessous ocreux.

S. FURGATA

$a^{\mathrm{d}}$ Front longuement gris olive, sourcils cendrés.

S. curtata

a" Dessous du corps maculé de noirâtre.

S. HYPOSTICTA

$b$ Sommet de la tête blanc lacté, ailes et queue rousses.

S. ALBicapilla

$c$ Parties supérieures du corps unicolores, queue rousse ou d'un roux noirâtre, gorge rousse ou maculée de noir.

c' Dessous blanc de crème, une grosse tache rousse varice de blanchătre au milieu de la gorge, queue brun noirâtre, à première rectrice toute rousse.

S. Arequipde

$c^{\prime}$ Dessous gris foncé, gorge ocreuse, queue rousse.

S. PUDIBUNDA

d Parties supérieures du corps striées ou tachetées.

d' Taches ou stries noires.

$d^{\text {a }}$ Tête fortement striée de noir, ailes et queue en partie rousses, dessous du corps fauve, tache mentonnière ocreuse variée de blanchâtre et de noir.

$d^{\mathrm{b}}$ 'Taches à peine distinctes sur la tête, plus prononcées et grosses au dos, rien de roux sur les ailes et la queue à l'extérieur, tache au menton d'un roux ferrugineux strié de blanc et de noir, des stries noires sur le devant et les còtés du cou.

\section{S. HUMILIS}

d" Stries longues blanches bordées de noir.

$d^{c}$ Gorge largement ocreuse unicolore.

S. graminigola

$d^{\mathrm{d}}$ Milieu de la gorge ocreux, strié de blanc. S. vingata

\section{Gente Phacellodonus}

a Front longuement roux, à baguettes des plumes rousses. 
$b$ Front longuement roux foncé, à baguette des plumes épaisse, paraissant ètre noire ou blanche selon la direction de la lumière.

P. STRIATICEPS

\section{Genre A UT'ONOLUS}

$a$ Parties supérieures du corps unicolores.

a' Gorge blanche, le reste du dessous gris isabelle, à flancs brunâtres.

A. Sclateri

$a$ " Gorge ocreuse, le reste du dessous fauve, à flancs brunâtres.

A. ochralenus

$b$ Sommet de la tète et dos striés de fauve.

b' Bec noirâtre, épais.

b" Bec gris corné, faible.

A. striaticeps

A. subulatus

\section{Gente PHILYdoR}

a Parties supérieures du corps brun roussâtre.

$a^{\prime}$ Les sourcils et tout le dessous d'un roux vif. Ph. Pyrrhodes

$a$ " Sourcils et gorge blanchâtres, dessous du corps gris brunâtre, stries blanches sur la poitrine.

Ph. montanus

a"" Sourcils et gorge jaunâtres, dessous du corps olive brunâtre, stries blanchàtres sur la poitrine.

Ph. striaticollis

$b$ Parties supérieures du corps olives.

$b$ ' Croupion plus ou moins roux.

$b^{a}$ Croupion roux intense, sourcils roussâtres, dessous du corps jaunâtre pâle.

$l^{\text {b }}$ Croupion roussâtre, dessous du corps fauve sale.

Ph. erythrocergus

Ph. subfulvus

b" Croupion concolore au dos.

$b^{c}$ Couleur olive du dos uniforme jusqu'aux sus-caudales.

Ph. subflavescens

$b^{\mathrm{a}}$ Couleur olive du croupion plus claire que celle du dos.

Ph. ruficaudatus

$c$ Parties supérieures du corps grises, ailes d'un roux cannelle à l'extérieur.

Ph. erythropterus

\section{Genre ANABAZENOPS}

a Dessus du corps brun olivâtre uniforme.

a' Sourcils d'un roux uniforme dans toute leur longueur.

A. RUfosuperciliaris

a" Sourcils roux au-dessus de l'œil, fauve blanchâtre derrière l'œil.

A. Cabanisi 
$b$ Dessus du corps brun roussâtre.

b' Sourcils jaune verdâtre devant l'oeil, ocreux vif en arrière.

A. temporings

b" Sourcils d'un roux ferrugineux, gorge ocreuse, côtés du cou roux.

A. RUFicollis

\section{Genre XeNOPS}

a Abdomen strié de blanc.

X. rutilue

$b$ Abdomen unicolore.

X. IITTORALIS

\section{GENRE SITTASOMUS}

$a$ Tête gris olive unicolore.

S. AMAZONUS

$b$ Tète à baguettes claires dans les plumes du sommet, des côtés et de la gorge.

S. stictol. ENus

\section{Genre natrgatrolrNis}

a Sourcil blanc jaunâtre, dos et queue rousse, gorge et taches du dessous blanc jaunâtre.

M. perlatus

$b$ Point de bande sourcilière, dos brun roussâtre, queue noirâtre, gorge et taches du dessous roux ocreux.

M. BRunnescens

\section{GENRE DENIDROCINCLI}

$a$ Côtés de la tète grisâtres, gorge d'une nuance plus pâle que celle de la poitrine et variée par les baguettes blanchâtres dans toutes les plumes.

D. FUMIGATA

$b$ Gorge blanchâtre traversée dans toute sa longueur par deux raies foncées.

D. MERULA

\section{GENRE DENDROCOLAP'TES}

a Tectrices alaires rayées de noir comme le dos. D. Raniolatus

$b$ Tectrices alaires variées de quelques macules noires et des stries fauves sur les baguettes. D. validus

\section{Genre Picolaptes}

$a$ Gorge largement d'un ocreux pur, de grosses stries isabelles sur le dessous du corps.

P. Souleyeti

$b$ Milieu de la gorge jaunitre, des stries fines blanchàtres, plus ou moins aiguës au bout sur le dessous du corps.

P. Wanszewiczi 


\section{GENTE IDENIDIROIRNIS}

a Ailes rousses presque en entier.

a' Taille forte, gorge ocreuse pure, taches ocreuses du dessous grosses et peu prononcées.

D. ROSTRIPALLENS

$a$ "Taille petite, dos brun olive varié de nombreuses stries fauves, gorge ocreuse squamulée finement de brun, nombreuses grosses taches fauves arrondies au bout sur la poitrine et subaiguës au ventre.

D. MULTigutTatA

$b$ Ailes rousses sur les rémiges, olives sur les tectrices.

b' Taches ocreuses du dessous grosses et subarrondies, taches du dos antérieur lacrymiformes.

D. ELEGANS

b" Taches du dessous lacrymiformes, celles du dos antérieur linéaires.

D. ocellata

c Peu de roussâtre sur l'extrémité des ailes, plumage général olive foncé, les taches jaunâtres de la poitrine assez grandes et subcarrées.

D. TRIANGULARIS

\section{Famile TYRANNID Æ}

\section{TRIBU TANIOPTERIN E}

A Queue fort arrondie, à rectrices étagées, couleur blanche prédominante.

Fluvicola

B Deux rectrices médianes atténuées et fort prolongées chez le mâle.

Copurus

C Queue à rectrices peu inégales.

C" Pattes élevées.

$C A$ Queue assez longue, aile longue.

Musgisaxicola

$C B$ Queue médiocre, scutelles du tarse soudées. Centrites

$C C$ Queue courte, huppe interne jaune.

Muscigralla

$C$ " Pattes médiocres.

CA Queue faiblement échancrée au milieu.

Ca Sourcil blanc ou jaune plus ou moins large.

$C_{\alpha}$ Corps plus ou moins trapu.

Ochthoeca 
$C \beta$ Corps svelte, coloration semblable aux Phyllopneustes.

Mecocerculus

Cb Sourcil peu marqué.

C๕. Queue rousse terminée d'une bande brune.

Myiotheretes

$\delta \beta$ Queue sans roux ou à barbe interne des rectrices en partie rousse.

Ochthodiata

$C B$ Queue à rectrices graduellement un peu plus longues depuis les externes aux médianes.

Ca Bec bicolore, plumage du mâle noir, à rémiges blanches intérieurement.

Cinipolegus

$C b$ Bec unicolore.

$C_{\alpha}$ Queue blanche en grande partie, narines percées dans une fosse.

Agrionvis

$C \beta$ Queue noire, narines percées sans fosse.

Arundinicola

\section{Genre MUSCISAXICOLA}

a Queue noire, à bord des rectrices externes blanc.

$a^{*}$ Front et sourcils blancs.

a a Point de tache nucale.

M. ALBIFRONS

$a b$ Tache nucale d'un roux clair.

M. FLAVINUCHA

a" Sourcils blancs.

aa Point de tache nucale.

$a_{\alpha}$ Sourcil très fin ne dépassant pas l'œil. M. cinerea

$a \beta$ Sourcil large dépassant l'œil, taille forte.

M. GRISEA

$a_{\gamma}$ Sourcil blanchâtre peu prononcé; base de la mandibule inférieure jaune.

M. MAGUlinostris

ab Tache nucale plus ou moins prononcée.

$a_{\alpha}$ Tache nucale d'un roux ferrugineux peu prononcée sur le reste du sommet de la tête gris roussâtre; sourcil blanc au-dessus de l'œil, cendré clair en arrière.

M. RUBricapiltaA

$a \beta$ Tache nucale d'un roussâtre faible, peu distinct de la couleur environnante; sourcil blanchâtre peu prononcé.

M. JUNinensis

$a_{y}$ Tache nucale d'un roux ferrugineux intense bien distinct de la couleur environnante; sourcil blanc très large. M. Rufrentex

a"" Point de sourcil blanc ni du blanc au front.

a Lores noirs, sommet de la tète brun roussâtre, du roux au menton.

M. nentalis 
$a b$ Sommet de la tête concolore au dos; gorge blanchâtre.

M. FLuviatilis

$b$ Rémiges et rectrices longuement rousses à la base de la barbe interne.

M. RUFipenNis

\section{Genre AGRIORNIS}

a Rectrices externes jusqu'à la quatrième inclusivement blanches en entier; la suivante blanche le long du milieu.

A. solitaria

$b$ La rectrice externe et la subexterne blanches avec une raie d'un brun terreux au bord du quart basal de la barbe interne, les trois suivantes terminées de blanc: la troisième dans les $2 / 3$ de la longueur; la quatrième dans la moitié; la cinquième dans le tiers.

A. ALBICAUd. $A$

$c$ Rectrices externes jusqu'à la cinquième blanches, la quatrième a une bordure grise sur la barbe interne, la cinquième bordée des deux côtés de cette couleur.

A. Pollens

d Rectrices externes blanches jusqu'à la cinquième, qui a le bord interne gris.

A. insolens

\section{GeNRE MYIOTHERETES}

a Le front et le croupion concolores au dos, la gorge et tout le devant du cou fortement striés de brun; bec épais.

M. STRIATICOLlis

$b$ Front longuement blanc, croupion roux, stries gulaires peu prononcées; bec faible.

M. ERYTHROPYGIUS

\section{GENRE OCHTHODI ATA}

a Queue unicolore; couleur du dessous du corps presque la même que celle du dessus.

O. Fumigata

$b$ Barbe interne des rectrices bordée largement de roux.

$b$ ' Ailes traversées de deux raies rousses, dessous du corps roux ocreux.

O. FUSCORUFA

$b$ " Deux raies transalaires fauves blanchâtres, milieu du ventre blanc jaunâtre.

O. SignatA

\section{Genre OCHTHOECA}

a Sourcils blancs.

$a^{\prime}$ Sourcils larges.

aa Point de bandes transalaires. 
$a_{\alpha}$ Dessous du corps gris cendré.

O. LEUCONETOPA

$a \beta$ Dessous du corps roux, à poitrine brunâtre.

O. POLIONOTA

ab Une bande transalaire rousse.

a $\alpha$ Abdomen roux jaunatre; croupion roussâtre; raie transalaire large.

O. OENANר HOÏDES

a $\beta$ Milieu du ventre largement blanc, poitrine d'un roux vif.

al Bande transalaire rousse large. O. Lessoni

$a 2$ Bande transalaire fine peu prononcée.

O. RUFipECTORALIS

$a$ " Sourcils fins.

aa Plumage général schistacé.

$a_{\alpha}$ Unicolore.

O. NIGRITA

a $\mathrm{A}$ grande plaque pectorale rousse.

O. THORACICA

$a$ "' Sourcils blancs, front jaune, ailes à deux

bandes rousses.

O. JeLSKII

$b$ Sourcils roussâtres, abdomen roux, deux bandes transalaires.

O. FUMICOLOR

c Sourcils blanchâtres peu larges; queue longue; deux larges bandes transalaires et bordures des rémiges secondaires rousses.

O. RUFimarginata

$d$ Sourcils jaunes.

d' Dos olivâtre, dessous jaune sulfureux pur.

$d^{\prime \prime}$ Dos roux olivâtre, dessous jaunâtre sale.

O. Salvini

O. GRATIOSA

\section{GeNRE MECOCERCULUS}

a Barbe interne des rectrices blanche.

a' Bordures des rémiges secondaires rousses. M. CALOpterA

$a^{\prime \prime}$ Bordures des rémiges secondaires jaunâtres. M. poecilocerca

$b$ Rien de blanc sur la barbe interne.

M. STICTOpterA

\section{TRIBU PLATYRHYNCHIN $Æ$}

A Bec assez court, presque aussi large que long, aplati, à arête dorsale proéminente dans toute sa longueur, terminée en crochet.

Platirimyehus

B Bec presque aussi long que la tête, aplati, large, linguiforme, subitement atténué au bout; queue à rectrices étroites, plus ou moins étagées.

Tonirostruy

G Bec semblable au précédent, mais plus fortement atténué dans sa partie terminale; rectrices étroites, peu inégales.

C' Point de huppe.

Euscarthaus

C" Plumes du sommet de la tête de plus en plus 
longues, en s'éloignant du front, noires bordées de cendré ou de roux, formant une huppe plate et large.

LOPHOTRIGGUS

C'" Bec plus graduellement atténué que dans les deux précédents; huppe interne rousse plus ou moins développée.

Hapalogergus

Orchiles

C"," Queue très courte, à rectrices fines.

D Bec plus ou moins fin.

$l^{\prime}$ Tète sans huppe.

DA Aile courte, à troisième et quatrième rémiges les plus longues, dépassant peu les secondaires; queue longue fort graduée.

Stiguatura

$D B$ Aile à troisième et quatrième rémiges les plus longues, dépassant fort les secondaires; queue médiocre, à rectrices peu inégales. SerphophaGa

$D$ "' Tète huppée.

DA Huppe composée de plumes effilées, plus ou moins prolongées.

An.eretes

DB Huppe plate, composée de plumes arrondies, larges, dont les médianes forment une huppe interne rouge semblable à celle des roitelets (Regulus); bec très fin.

Cyanotis

\section{Genre PLATyriy ychus}

a Sommet de la tête schistacé, à huppe interne blanche.

P. SENEX

$b$ Sommet de la tête concolore au dos, à huppe interne jaune.

P. albigularis

\section{Gente TODIROSTRUM .}

a Bande sourcilière jaune.

b Gorge et poitrine striées de noir; jooint de tache blanche occipitale.

1. CHRYSOCOTAPHUN

Point de bande sourcilière, ni stries en dessous.

c' Dos cendré, dessous jaune.

ca Gorge jaune.

$c b$ Gorge blanche.

T. CINEREUN

T. SGlateri

c" Dos noir, dessous jaune; petites tectrices alaires marron foncé, les grandes et les moyennes jaunes.

T. PULGhellui

\section{Genre LOPHOTIRICCUS}

a Plumes du sommet de la tète noires bordées de roux.

$b$ Plumes du sommet de la tète noires bordées de cendré.

L. SPICIFER 


\section{GeNRE EUSCARTHMUS}

$a$ Dos vert olive.

a' Gorge noire, tour de l'œil roux.

E. PYRRHOPS

$a$ " Gorge et cou roussâtres, sommet de la tête

lavé de roussâtre.

E. rufigularis

a"' Gorge, devant du cou et milieu de la poitrine

blanc pur, sommet de la tête gris foncé. E. RuFipes

$b$ Sommet de la tête et dos gris foncé, croupion olivâtre; gorge blanc soyeux strié de grisâtre.

E. Wughereri

\section{Gente HAPALOCERCLS}

a Tour de l'œil largement roussâtre, un peu de roux sur la base des plumes occipitales.

H. FULViceps

$b$ Tour de l'œil olive, tout le dessous soufré.

H. ACUTIPENNis

\section{Genre SERPIIOPHAGA}

a Point de huppe.

a' 'Tête avec la gorge rousse.

S. RUFicEPS

$a$ " Tête noire en dessus.

S. CINEREA

$b$ Petite huppe noire verticale.

S. HYPOLEUCA

\section{Genre AN ERETES}

a Ventre jaune.

$a^{\prime}$ Plumes de la huppe recourbées en avant.

A. parulus

$a$ " Plumes de la huppe droites.

A. AgILIS

$b$ Plumes de Ja huppe longues et droites.

b' Abdomen jaunâtre.

A. albocristatus

$b$ " Abdomen blanc.

A. nigrocristatus

\section{TRIBU ELAINEIN $A$}

A Bec plus court que la tête, non èlargi et non aplati à la base, régulièrement atténué ver's l'extrémité. $\Lambda^{\prime}$ Bec à arête dorsale distincte jusque près de l'extrémité; cils maxillaires petits et peu nombreux; quatrième rémige la plus longue; première et septième, base de la mandibule rouge. Mionectes

$A$ " Bec à arête dorsale arrondie; cils maxillaires longs et abondants; cils petits nombreux sur les joues.

AA Bec plus long que la moitié de la tête. 
$A B$ Bec à peine plus long que la moitié de la tête.

Pogonotriccus

A" "Bec assez large à la base, graduellement atténué vers l'extrémité; cils longs et abondants; troisième et quatrième rémiges les plus longues. Capsievpis

B Bec plus court que la tête, triangulaire, assez large et non aplati à la base, à ligne latérale droite, arête dorsale anguleuse.

B' Bec court.

B" Bec plus long que la moitié de la tête.

Phyllomias

Myiopatis

C Bec fin, comprimé devant les narines, à arête fort proéminente dans la partie basale; cils maxillaires fins et peu nombreux; deuxième rémige la plus longue.

Ornithion

D Bec court subtriangulaire, assez large ả la base.

D' Cils maxillaires assez forts et nombreux; point de huppe interne.

Tyranniscus

D" Cils maxillailes très petits et peu nombreux, une huppe interne jaune.

Tyrannulus

D"'Cils maxillaires assez nombreux; le plus souvent une huppe interne blanche ou jaune.

Elainea

E Bec à côtés concaves, fort comprimé à l'extrémité terminée par un crochet long; huppe interne d'un rouge orangé.

Myozetetes

F Bec à côtés légèrement convexes, large à la base, n'est comprimé qu'à l'extrémité même en crochet fort courbé; point de huppe interne.

Conopias

G Bec court, triangulaire, large à la base, à arête dorsale arrondie, l'extrémité même comprimée; cils peu nombreux et peu longs.

$G$ ' Huppe interne jaune.

Legatus

G" Point de huppe interne.

Sublegatus

H Bec court, large, aplati à la base, à côtés plus ou moins convexes, terminé en crochet.

Rhynghogyglus

I Bec considérablement plus long que le tarse, gros, plus large que haut, terminé en crochet fort courbé; cils maxillaires longs et assez abondants.

I' Ligne latérale du bec presque droite jusqu'au crochet.

I" Ligne latérale du bec convexe.

$I A$ Huppe interne jaune.

$I B$ Point de huppe interne.

Pitangus

Myiodynastes

SiRYSTES

\section{GeNre MIONECTES}

a Dessous roux, sommet dela tète concoloreau dos. M. oleagineus

$b$ Abdomen jaune soufré; tête d'un cendré ardoisé, des stries sur la gorge et la poitrine.

M. striaticollis 


\section{GeNRE LEPTOPOGON}

$a$ Còtés de la tête maculés de blanc.

$a^{\prime}$ Sommet de la tête schistacé, à front parsemé de blanc; une tache auriculaire noirâtre. L. Superciliaris

a" Sommet de la tête brun café; front non tacheté; plumes alaires bordées de roussâtre; tache auriculaire brun foncé.

L. PERUTiLuS

a" Sommet de la tète schistacé olivâtre, à front non tacheté; le devant du cou et la poitrine d'un roussâtre sale.

L. RUFIPEGTUS

a"' Sommet de la tête ardoisé, à front immaculé, des stries blanches très petites audessous de l'œil; point de tache auriculaire foncée.
L. MINOR

\section{Gente MYIOPATIS}

a Abdomen jaune.

M. $W_{\triangle G . E}$

$b$ Abdomen blanc.

M. TUMBEZZANA

\section{Genre ORNITHIION}

$a$ Dessus gris avec une teinte olivâtre très faible; dessous blanchâtre lavé souvent d'isabelle.

$b$ Dessus olive sale, dessous jaune soufré pâle.

O. Sclateri

O. pusillusi

\section{GeNre TYRANNISCUS}

a Front jaune, sommet de la tête concolore au dos.

T. CHRYsOpS

$b$ Devant du front blanchâtre, sommet de la tête ardoisé olivâtre.

T. VIRIDIFLAYUS

$c$ Sommet de la tête noir.

T. Nigricapillus

$d$ Sommet de la tête cendré, côtés de la tête maculés de blanc; une grosse tache auriculaire noire.

T. GINEREICLPS

$e$ Sommet de la tête cendré, à disque des plumes plus foncé.

T. GRAGILIPES

$f$ Sommet de la tète olive verdâtre concolore au dos.

T. viridissinus

\section{Genre Rla INEI}

a Huppe interne blanche.

a' Dos gris olive. 
a a Milieu de l'abdomen blanc; huppe interne fort développée.

$a_{\alpha}$ Gorge gris cendré; point de sourcil blanc.

E. ALBICEPS

$a \beta$ Gorge blanche; sourcil blanc dépassant peu l'œil.

E. Leucospodia

ab Milieu de l'abdomen jaune soufré.

au Huppe interne fort dévelopjée.

al Taille forte; gorge largement blanche, bandes transalaires blanchâtre sale.

E. GIGAS

$a$ ? Taille médiocre, gorge jaunâtre; bandes transalaires blanc presque pur.

E. PALLATANGe

a $\beta$ Huppe interne peu développée, quelquefois nulle; gorge blanchâtre; bandes transalaires blanchâtre sale.

E. PAGANA

$a$ " Dos olive verdâtre; milieu de l'abdomen jaune; bandestransalaires jaune pâle; sommet de la tête noiràtre.

E. ELEgans

$b$ Huppe interne jaune citron.

b' Sommet de la tête ardoisé noirâtre; bandes transalaires grises peu prononcées.

E. SUbPLACENS

b" Sommet de la tête olive plus foncé que le dos, deux larges bandes jaunâtres en travers de l'aile.

E. CANICEPS

$c$ Point de huppe interne.

c' Taille forte; tout le dessus olive brunâtre, dessous jaunâtre, deux larges bandes transalaires blanchâtre sale.

E. Glgas

c" Taille petite; sommet de la tête gris olivâtre concolore au dos, à plumes graduellement prolongées en formant une huppe assez élevée.

ca Trois bandes transalaires blanc sale, croupion roussâtre.

E. BREVIROSTRIS

$c b$ Deux bandes blanches en travers de l'aile, croupion olivâtre.

E. GRAGILIS

\section{GeNRE MYIOZE'TEIES}

$a$ Sourcils blancs plus ou moins larges.

a' Bordures des rémiges primaires rousses; bandes sourcilières réunies sur la nuque, lıuppe interne orangée rougeâtre bordée largement de jaune.

M. GAYENNENSIS 
$a$ " Bordures des rémiges primaires jaune olivâtre.

aa Huppe interne rouge orangé, bordée finement de jaune; plumes du sommet de la tête olive foncé; front peu enduit de blanchâtre.

M. SIMILIS

$a b$ Huppe interne orangée rougeâtre; sourcils très larges, front longuement blanchâtre; plumes du sommet et des côtés de la tête d'un cendré ardoisé.

M. GRANADENSIS

$b$ Sourcils nuls.

$b$ : Tête cendrée, à huppe interne orangée en entier chez le mâle, jaune citron chez la femelle; gorge et devant du cou largement blancs.

M. SULPHUREUS

$b$ " 'Tête d'un olive très foncé, à huppe interne d'un minium orangé bordée de plumes blanches en partic jaunes.

M. LUTEIVENTRIS

\section{Genre RIYYCHOCYCLUS}

a Queue olivâtre.

a' Sommet de la tête concolore au dos.

$a a$ Cou antérieur et poitrine d'un roux olivâtre; plumes alaires bordées de roussâtre.

R. FULVIPECTUS

$a b$ Poitrine jaunâtre pâle.

R. VIRIDICEPS

$a$ " Sommet de la tête cendré.

aa Sommet de la tête cendré bleuâtre; tache auriculaire noirâtre très prononcée; longueur de l'aile, 74 millimètres.

R. peruvianus

$a b$ Sommet de la tête schistacé enduit plus ou moins d'olive; tache auriculaire nulle; longueur de l'aile, 61-66 millimètres.

R. SUlPHURESGENS

ac Sommet de la tête ardoisé enduit légèrement d'olivâtre; tache auriculaire nulle; longueur de l'aile, 53 millimètres.

R. MEgAriephaLUS

ad Sommet de la tête cendré, à plumes enduites de vert olive sur leurs extrémités; longueur de l'aile, 53 millimètres.

R. POLIOGEPHALUS

$b$ Queue rousse; plumes alaires bordées largement de roux; dessous du corps fort strié.

R. RUFiGAUDA

\section{Gente MYIODYNaSTES}

$a$ Corps fort tacheté.

$a$ ' Le brun foncé occupant largement le milieu 
des rectrices; tout le milieu du ventre tacheté; une bande foncée large au milieu des sous-caudales.

M. solitarius

$a$ " Le brun foncé occupant finement le milieu des rectrices.

aa Menton blanc, moustache brune fine, milieu du ventre blanc pur; bordures des rémiges primaires rousses.

M. ALDAX

$a b$ Menton noirâtre prolongé en moustaches larges; milieu du ventre blanc plus ou moins enduit de jaune; sous-caudales blanches ou jaunes, à baguettes blanches en grande partie.

M. LUTEIVENTRIS

$b$ Corps non tacheté.

b' Dos olive; queue schistacée, à bordures olives fines.

M. CHRYSOCEPHALUS

b" Dos brunâtre; queue en grande partie et croupion roux.

M. ATRIFRONS

\section{TRIBU TYRANNIN}

A Bec depuis la commissure plus long que le tarse. $A^{\prime}$ Bec beaucoup plus long que le tarse.

$A A$ Bec aplati et large, cils maxillaires prolongés jusque près de son extrémité; tête ornée d'une huppe en éventail transversal. Muscivora

$A B$ Bec large non aplati, cils maxillaires courts; tête ornée d'une huppe interne jaune.

Megarhynghus

$A$ " Longueur du bec dépassant peu celle du tarse.

$A A \mathrm{Bec}$ aplati à la base, subtriangulaire, comprimé à l'extrémité, à crochet terminal fort courbé, la ligne latérale convexe à la base et concave à l'extrémité.

Aa Cils maxillaires n'atteignant pas la moitié du bec, la deuxième rémige la plus longue, la troisième distinctement plus courte; couleur du dessous du corps rousse.

Hirundinea

$A b$ Cils maxillaires dépassant la moitié du bec; les deuxième et troisième rémiges les plus longues et égales.

$A B$ Bec triangulaire non aplati à la base, à côtés presque droits, terminé par un crochet courbé.

A $a$ Bec faible; sommet de la tête à plumes allongées, formant une couronne abondante; coloration différente dans les deux sexes.

$A b$ Bec épais, large à la base. 
$A_{\alpha}$ Point de huppe interne.

Mriarches

A $\beta$ Une huppe interne jaune ou orangée.

Al l'remière rémige atténuée fai-

blement à l'extrémité, les autres normales; bec plus court que la tête.

$A 2$ Six rémiges primaires fort atténuées à l'extrémité.

Empidonomus

A3 Deux rémiges externes fort atténuées à l'extrémité; queue très longue et très profondément fourchue.

Tyrannes

Milvulus

B Bec depuis la commissure plus court que le tarse.

B' Huppe interne jaune ou rouge, queue beaucoup plus courte que l'aile.

Myiobius

$B$ " Huppe interne peu prononcée; queue à peine moins longue que l'aile.

$B$ " Point de huppe interne.

$B A$ Troisième et quatrième rémiges les plus longues.

Mitrephores

$B B$ Deuxième et troisième rémiges les plus longues.

Empinochanes

Empidonax

\section{Genre CONTOPUS}

$a$ Plumage général ardoisé, moins foncé en dessous; taille forte.

C. ardesiacus

$b$ Couleur du dessus du corps gris olivâtre, à sommet de la tête plus foncé.

b' Taille forte; milieu du dessous jaunâtre pâle, flancs largement gris olive strié de plus foncé; longueur de l'aile, 113 millimètres.

C. Borealis

b" Taille petite.

ba Sommet de la tête noirâtre; bec fort aplati à la base, à arête dorsale fort proéminente; longueur de l'aile, 72-74 millimètres. C. Punersis

bb Sornmet de la tête brun; arête dorsale du bec presque effacée.

$b_{a}$ Longueur de l'aile, 83 millimitres. C. PLebesus

b $\beta$ Longueur de l'aile, 74 millimètres. C. virens

\section{Gente Pyirocephalus}

a La tête et tout le dessous rouges chez le mâle. $a$ ' Taille plus forte; longueur de l'aile, 80 millimètres. 
$a$ "Taille moins forte; longueur de l'aile, 74-

75 millimètres.

P. Rubinets

$b$ La tête et le dessous d'un fuligineux lavé de rosé.

P. obscurus

\section{GenRe MYIOBIUS}

a Huppe interne dans les deux sexes.

a' Dessus du corps olive, croupion et ventre

d'un jaune pâle.

aa Poitrine et flancs largement roux, huppe interne jaune chez le mâle, rousse chez la femelle; bec large.

M. villosus

$a b$ Poitrine lègèrement enduite de roussâtre; bec peu élargi.

$a$ "Tout le dessous roux vif; dos olive foncé; une bande ocreuse en travers du croupion; huppe jaune citron dans les deux sexes.

M. cinnamoneus

$a$ "' Dessus olive verdâtre, croupion et ventre largement jaunes; queue rousse unicolore; huppe interne jaune.

$a$ "'" Dessus olive foncé, dessous jaune serin, à poitrine enduite de roussâtre; huppe interne roux orangé.

M. Barbatus

"'" Espèces tachetées en dessous.

aa Dessous fort strié.

$a_{\alpha}$ Fond du dessous blanc; huppe interne roux cannelle vif; souvent jaune chez la femelle.

M. CRYPTERYTHRUS

$a \beta$ Fond du dessous jaunâtre pâle; huppe. interne jaune.

M. Phoenicurus

M. PULGHER

M. NeviUs

$a b$ Dessous roux non strie; huppe interne roux cannelle.

M. RUEESGENS

$b$ Huppe interne chez le mâle, nulle chez la femelle; olive verdâtre en dessus, jaune en dessous; tour de l'œil jaune; point de bandes transalaires.

M. SUPERCILIOSUS

c Point de huppe interne dans les deux sexes, queue, ailes et dessous du corps roux.

M. enythrurus

\section{GENRE EMPIDOCHANES}

a Barbe interne des rémiges et des rectrices bordée largement de roux; ventre, sous-caudales et sous-alaires roux.

E. poecilurus

$b$ Queue schistacée; milieu du ventre jaunâtre pâle; deux bandes transalaires et bordures des rémiges roussâtres.

E. olivus 


\section{GenRe MYIARCIUS}

a Poitrine cendrée; abdomen jaune pâle.

a' Barbe interne des rectrices largement rousse. M. en Ythrocercus

$a "$ Point de roux sur la queue.

aa Sommet de la tête noir.

M. NIGRICEPS

$a b$ Sommet de la tête cendré, à milieu plus

ou moins noirâtre.

M. PH. EOCEPHALUS

ac Sommet de la tête brun olivâtre.

$a_{\alpha}$ Barbe externe des rectrices latérales grise, bandes transalaires peu prononcées.

M. TYRanNulus

$a \beta$ Barbe externe des rectrices latérales blanche ou blanchâtre; bandes transalaires bien prononcées.

M. CEPHALOTES

$b$ Tout le dessous du corps roux.

M. SEMIRUfus

\section{Genre TYRANNUS}

a Abdomen jaune.

a' Gorge cendrée, poitrine olivâtre; huppe interne orangée bordée de jaune; queue profondément échancrẻe.

T. MELANGHolicus

$a "$ Gorge blanche passant au cendré clair sur la poitrine; huppe interne jaune citron; queue à peine entaillée.

T. niveigulanis

$\checkmark$ Abdomen blanc; tête noire au sommet et sur les côtés, huppe interne orangée bordée de blanc; queue terminée de blanc.

T. PIPIRI

$c$ Tout le dessous gris cendré; sommet de la tête noir; huppe interne jaune citron.

T. AURANTIO-ATROGRISTATUS 


\section{linilite PIPRIDE}

A Rémiges primaires atténuées aiguës à l'extrémité.

A' Primaires jusqu'à la cinquième ou sixième lon- guement atténuées, plus ou moins courbées, les secondaires raides.

Chiromacheris

A" Primaires jusqu'à la troisième ou quatrième atténuées jusqu'à l'extrémité, droites.

Chiroxiphis

A"'Premiòre et deuxième rémiges longuement atténuées à l'extrémité; dessous du corps strié. MaGh kropterus

B Rémiges primaires normales.

B' Queue courte, raide ou molle, quelquefois à rectrices prolongées en appendices criniformes. Pipra

B" Queue longue, étagée; plumes frontales érigées, piliformes.

Metopothrix

B" Queue médiocre; coloration modeste.

BA Queue coupée carrément ou légèrement arrondie.

$\mathrm{Ba}$ Bec médiocre turdiforme.

Heteropelia

Bb Bec court assez robuste.

SChifFornis

$B c$ Bec court et faible.

Ba Troisième et quatrième rémiges les plus longues et égales.

Chloropipo

B $\beta$ Troisième rémige la plus longue. NEoprpo

$B C$ Queue plus ou moins ètagée.

$\mathrm{Ba}$ Queue cunéiforme, à rectrices terminées par une tache blanche.

Piprite:

$B$ b) Queue à six rectrices médianes larges presque égales, les autres fort étagées et étroites.

Heterociercus

\section{GeNRE PIPRA}

a Queue à baguettes des rectrices prolongées en un appendice criniforme plus long que les rectrices mèmes, et courbées; tète et cou postérieur du màle rouge écarlate, tout le dessous jaune.

P. FiLicalid

$b$ Queue normale courte.

$b$ Tète à huppe cervicale bifide; tète rouge. P. connuta

b" Tète sans huppe.

ba Queue à rectrices raides, les baguettes epaisses et raides.

$b_{\alpha}$ Tète róuge, àgorge noire, tibias jaunes. P. ChLoroyeros 
$b \beta$ Tête jaune, à gorge noire, tibias rouges. P. Auricapilla $b b$ (jueue à rectrices non raides.

$b_{\alpha}$ 'Tète rouge, à gorge noire; tibias blancs avec du rouge sur le bas du côté externe.

P. RUBRicapilla

$b \beta$ Vertex, nuque et derrière du cou rouges; front, côtés de la tète, gorge et devant du cou jaunes.

P. FAsciata

by Sommet de la tête bleu.

$b 1$ Front concolore au reste du sommet; le croupion et les sus-caudales bleus.

P. C.ERLLEOCAPILla

$b 2$ Front noir, point de bleu au croupion.

P. cyaneocapilla

$b \delta$ Sommet de la tête blanc.

$b 1$ Croupion et sus-caudales bleus. P. Isinon

b2 Point de bleu au croupion.

$b^{\star}$ Taille plus forte, le blanc plus prolongé sur la nuque; longueur de l'aile, 65 millimetres.

P. CORACIN.I

$b^{\star \star}$ Taille moins forte, le blanc moins prolongé sur la nuque; longueur de l'aile, 59 millimètres.

P. Leucocillis

$b \varepsilon$ Plumage vert olive dans les deux sexes. P. virescers

\section{GENRE IACII EROPTERUS}

a Sommet de la tête jaune aver une raie médiane rouge.

M. pyrocephalus

$b$ Sommet de la tête rouge.

M. STRIOLATUS

\section{Gente IIETEROPELMA}

a Dessus olive sale, presque uniforme partout.

H. WaLlaciI

$b$ Dessus olive brunâtre, à sommet de la tète d'une nuance différente; bordures des plumes alaires roussâtres.

H. Amizonum 


\section{FaimLLe: COTINGIDAE}

\section{TRIBU TITYRIN $Æ$}

A Deuxième réntige du mile normale; plumage général cendré avec du noir et du blanc. Titvia

B Deuxième rémige du mâle courte et aiguë au bout. l' Cils maxillaires assez forts; plumage du mâle cendré foncé sans blanc aux ailes et la queue, roux dans la femelle.

Hadrostomus

B" Cils maxillaires faibles et courts; plumage du màle cendré, noir ou vert, souvent avec du blanc aux ailes et sur la queue; coloration différente dans les femelles; rarement rousse dans les deux sexes.

Pachyrihaphus

\section{Tribu LIPAUGIN $\boldsymbol{E}$}

A Cils maxillaires forts.

A' Ligne dorsale du bec droite jusqu'à l'extrémité courbée en crochet; narines rondes; talon armé de squamules disposées en scie.

LipaUGUS

A" Ligne dorsale du bec courbe; narines larges en travers; talon sans armure.

LATHRIA

B Cils maxillaires courts et fins; ligne dorsale du bec droite jusqu'à l'extrémité courbée en crochet. Aulr.

\section{TRIBU ATTILIN $\approx$}

Ligne dorsale du bec droite, jusqu'au bout courbé en crochet; cils maxillaires forts, mais courts.

Attilia

\section{Tribu RUPICOLIN $\approx$}

A Bec courert en entier par les plumes frontales érigćes en une crète rerticale fort élevée.

Repicola

B Plumes frontales courtes et verticales, le reste du plumage du sommet de la tite plat.

Phoevicocercus 


\section{TRIBU COTINGIN $\nexists$}

A Tète sans huppe.

A' Plumage général vert.

$A A$ Troisième et quatrieme rémiges les plus longues et égales.

Ampelio

$A B$ Quatrième et cinquième rémiges les plus longues et égales.

Pipreola

A" Rémiges primaires externes atténuées; tectrices alaires non atténuées; narines découvertes.

\section{Cotivga}

A"'Rémiges primaires non atténuées.

A Taille forte, narines couvertes en entier par les plumules, ailes et queue à lustre coracin. Sericossipha

$A B$ Taille petite, narines couvertes par les plumules criniformes, plumage modeste, ì faisceau de plumes violettes sur les flancs. Iodopleura

B Huppe occipitale assez longue, composée de plumes atténuées, rousses en grande partie.

Heliocheri

C Huppe interne rouge cannelle.

DOLIORNIS

\section{TRIBU GYMMODERIN $巴$}

A 'Tète surmontée d'un panache élevé abritant toute la tète et le bec, une expansion cutanée plus ou moins longue au-dessous de la gorge, emplumée ou dénuée.

Cephalopterus

B Tête sans huppe.

P' Toute la tète et le cou emplumés, narines courertes de plumes frontales.

$B A$ Bec fort élargi, aplati, deux fois aussi large que haut derrière les narines, troisième et quatrième rémiges égales et les plus longues. Qterula

$B P$ Bec peu élargi et non aplati, moins de deux fois aussi large que haut, derrière les narines; quatriène et cinquième rémiges égales et les plus longues.

Pirodents

I)" Joues et còtés du cou dénuís en grande partie; narines non couvertes; deuxième rémige la plus longue.

Grunonerus

\section{GeNre TITYYR A}

a Femelle striée de noir; le noir du sommet de la tète chez le mâle prolongé jusqu'à la nuque.

T. GAYANA

b) Femelle non striée de noir. 
b' Queue blanche à la base et à l'extrémité.

b)" Queue toute noire.
T. SEMIFASGIATA

T. ALBITORQUES

\section{GFARE II ADROSTOUUS}

a Mâle, à cravate rosice en travers du cou.

H. $\mathrm{MINOR}$

1) Point de rosé au cou.

b' Mâle, à dessous du corps gris pâle. H. AUDAx

b" Mâle, à dessous du corps schistacé cendré. H. Hovochrous

\section{Gente PaChYRHAMpilus}

$a$ Sommet de la tète noir chez le mâle.
a' Dos du màle vert olivâtıe.
P. VIRIDIS

$a^{\prime \prime}$ Dos du màle cendré.

aa Rectrices terminées longuement de blanc. P. albogriseus

$a b$ Rectrices bordées fincment au bout de blanchâtre.

P. CINEREUS

$a^{\prime \prime}$ Dos du mâle noir.

a Rectrices terminées longuement de blanc.

au. Dessous du corps cendré ardoisé. P. atricapillus

aß Dessous du corps noirâtre. P. Niger

ab Rectrices non terminées de blanc.

ac Dessus du corps cendré ardoisc. P. sponteres

a $\beta$ Dessous blanchàtre, à cou jaune, le
tout ondulé de foncé.
P. VERSTOLOR

b Siommet de la tète roux dans les deux sexes, plumage général roux.

P. RUFESGENS

\section{Genre A'TILI}

a Sommet et còtés de la tète cendré obscur, croupion et milieu de l'abdomen jaune roussàtre clair. A. citrintrentris

$b$ Sommet de la tète strié finement de foncé, milieu de l'abdomen jaunàtre.

A. TORRIDUs

$c$ sommet de la tête aussi roux que le dos, rien de jaune ni au croupion ni au milieu du ventre. A. Thumvormuönes

\section{GEXRE PIPIREOLI}

a Capuchon céphalique.

a' Vert obscur entouré d'une hordure jaune; le jaune des flancs strié de vert.

P. Riefferi

a" Noir entouré d'une bordure jaune; le jaune de l'abdomen squamulé de noirâtre. 
a" Noir non bordé de jaune; milieu de l'abdlomen jaune pur, flancs verts.

P. LubomirskiI

$b$ Capuchon céphalique peu prononcé et réduit audevant du visage, plaque gutturale d'un orangé vif.

P. elegans

\title{
Genke A MPELIO
}

a Dos vert olivâtre unicolore, dessous rayé en travers de noir; capuchon noir chez le mâle.

$b$ Plumes du dos noires bordées de vert olive, celles dı dessous vert olives, à disque jaune; chez le nıâle sommet de la tête noir, lores et demi-collier nucal jaunes.

A. ARGuites

\author{
A. ARGLATLS
}

A. cinctus

\section{GeNRE HELIOCHERA}

a Rectrices à tache blanche subterminale, point de collier roux.

H. Rubrochistata

b liectrices sans tache blanche, collier rowx oecupant la gorge, région auriculaire et la nufue.

II. RUFAXILLA

\section{Genre Co'TINGA}

a Dessus du corps bleu.

a' Bleu uniforme sur tout le dessus, tectrices alaires bleues, gorge largement violette. C. махмаNa

$a$ "Base des plumes bleues longuement noires, tectrices alaires noires bordées de bleu, gorge et devant du cou violet.

C. CAYAXI

b Dessus du corps noir squamulé de blanc, dessous blanc, gorge violet obscur.

C. PORPHYROL. WHA 


\section{FanilLe PHYTOTOMIDE}

\section{GeNie PII Y'TOTON}

a Tout le sommet de la tète roux.

PH. RARA

$b$ Le roux n'occupe que la bordure frontalc.

Ph. Ramilondi

\section{FaMiLLE CORVID瓜}

A Plumes frontales plus ou moins longues et érigées en y formant une espèce de huppe.

Cyanociurax

B Plumes frontales courtes sans former de huppe.

Cyanogitta

\section{Gente CY MOCORAX}

a Plumage du corps violàtre, tête et devant du cou noirs.

C. violaceus

6 Dessus du corps bleu foncé, tête et devant du cou noirs; la nuque, le cou postérieur, le dessous du corps et les rectrices latérales blancs.

C. MYst.aciths

c Dessus du corps rert, dessous jaune, sommet de la tête blanc jaunàtre.

C. YNois

\section{Genre CYA MoCit'TA}

a Plumage général bleu verdàtre, plaque gulaire d'un vert très obscur, entourée en dessous d'une bordure blanche.

C. virinigyanea

$b$ Plumage général bleu outremer, plaque gulaire bleu violàtre, à bordure blanche peu prononcée. C. JoLles 


\section{FaniLLe ICTERIDE}

A Bec épais, large à la base, à arète dorsale fort dilatée en une scutelle plus ou moins élevée audessus du front.

A' Scutelle frontale renflée.

AA Ailes n'atteignant qu'à la moitié de la queue. Clypeigterus

AB Ailes dépassant l'extrémité de la queue. Ocyalus

$A$ " Scutelle frontale plus ou moins aplatie.

$A A$ Queue longue plus ou moins étagée. Ostrops

$A B$ Queue médiocre arrondie à l'extrémité. Cissıcus

B Bec moins fort, à arête dorsale non élargie.

$B^{\prime}$ Tête tout emplumée.

$B A$ Queue assez longue, ì rectrices latérales fort raccourcies; couleur prédominante jaune.

ICTERUS

BB Queue médiocre, à rectrices externes peu raccourcies.

XnNthosones

BC Queue médiocre coupée carrément à l'extrémité.

$B a$ Bec conique élevé à la base; beaucoup de rouge sur le dessous du corps.

$B \propto$ Sourcils blancs chez le mâle.

Trupialis

$B \beta$ Point de sourcil blanc chez le mâle. Leistes

$B b$ Bec assez faible, point de rouge. Agelalus

$B c$ Plumage noir uniforme.

$B \alpha$ Bec court épais.

B1 Queue coupée carrément.

Molothres

$B$ : Queue arrondie.

$B^{\star}$ Troisième, quatrième, cinquième et sixième rémiges les plus longues.

Dives

$B^{\star \star}$ Deuxième et troisième rémiges les plus longues.

LAMPROPSAR

B3 Rectrices prolongées en pointe. Dolichonrx

B" Lores, tour de l'œil et côtés de la gorge dénués. Grunomstax

G Bec très épais, large à la base, à arête dilatée en une scutelle frontale aplatie; deuxième rémige la plus longue; plumage noir coracin.

Cassidix

D Bec à arête non élargie à la base; troisième rémige la plus longue; queue cunéiforme; plumage noir, à reflets bleus ou verts.

QuisGalus

\section{Genre OStitiops}

a. Dos, ailes et rentre malron, tète, cou et poitrine jaune olivâtre.

o. Iuragarium 
b Couleur noire prédominante au corps.

O. Degumanus

c Couleur olive prédominante au corps.

c' Plaque frontale élargie.

O. VIRIDIS

$c^{\prime \prime}$ Plaque frontale peu large.

ca Bec jaune.

ca Quatre rectrices médianes toutes olives. O. ATrovirens

$c \beta$ Deux rectrices médianes et la barbe in-

terne des submédianes olives; cotés du

front d'un jaune sulfureux. O. Alfredi

cb Bec noirâtre.

O. ANGUSTIFrons

\section{Genke Cassicus}

"Noirs, à croupion jaune.

$a^{\prime}$ Rectrices jaunes à la base.

$a a$ Dans la plus grande moitié. $\quad$ C. persicus

ab Dans la plus petite moitié. C. Flavicrissus

$a$ " Rectrices noires en entier.

aa Ailes toutes noires. C. chrysunotus

$a b$ Grosse tache jaune sur les tectrices alaires. C. Leucorhampius

1) Noirs à croupion rouge, plumage luisant. $\quad$ C. afFinis

c Noirs en entier.

c' Troisième et quatrième rémiges les plus

longues; base du bec plombé olivatre. $\quad$ C. solitarics

c"Quatrième et cinquième rémiges les plus

longues, bec tout jaune. C. Holosericeus

\section{Gente IC'TER US}

" Plumage noir, à tache humérale jaune.

a' Sommet de la tête, croupion et tibias jaunes. I. CHnssocepindl's

a" Sommet de la tète, croupion et tibias noir's. I. carennensis

b Plumage jaune en grande partie, région inter'scapulaire noire.

$b$ ' Rectrices noires, l'externe blanche à l'extérieur.

I. GRACE-ANTE

b" Rectrices médianes noires, les externes jaunes.

I. MESOMELAS

c Plumes de la gorge longues et atténuées.

Plumage orangé, à dos concolore, queue toute

noire.

I. cROCONOTUS

\section{GENRE TRUPIALIS}

a Sous-alaires blanches.

a' Bec dépassant 3 centimètres; tibias gris, tachetés de noir.

T. MILIT.ARIS

(a" Bec n'atteignant pas 3 centimètres; tibias blancs.

T. BELLIGOSA 


\section{FanilLe TANAGRID在}

\section{TRIBU EUPHONIIN $\approx$}

A Coloration dissemblable dans les deux sexes.

A' Bec deux fois aussi large à la base que haut; queue médiocre, faiblement échancrée.

Procmias

A" Bec un peu plus large ì la base que haut; queue courte.

A A Plumage général vert vif, à éclat vitreux, dessous jaune; femelle peu différente.

Chlorophona

AB Plumage du mâle noir lustré de bleu violet, à dessous jaune ou roux, femelle olive; ou mâle olive peu différent de la femelle.

Euphonil

B Coloration semblable dans les deux sexes; bec aussi haut que large à la base, queue médiocre.

Pipridea

\section{Gexre IUPIIONIA}

a Màle noir lustré de bleu en dessus.

$a^{\prime}$ sommet de la tête bleu.

E. NigRicolis

a" Tète et gorge tout noirs.

E. RUFIVENTRIS

a" Sommet de la tète plus ou moins jaune.

aa Queue noire en entier.

$a_{\alpha}$ Le jaune du sommet de la tite prolongé jusqu'ì la nuque; gorge noire. E. saturata

a $\beta$ Le jaune occupant le front et le vertex; gorge jaune.

E. MELANLRI

ab Rectrices externes à tache blanche.

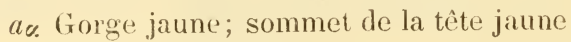
jusqu'à la nuque.

E. Hгрох.мтн1

$a \beta$ Gorge noire.

al Lustre violet sur la tète et le cou.

1 Le jaune frontal et du dessous clair.

E. serminostris

$\approx$ Le jaune frontal et du dessous orangé sale.

E. XANTHOG.ASTRA

$a$ ? Lustre de la tête et du cou non violet.

E. Minuta

6 Màle vert olive en dessus.

b) Front d'une nuance tirant au jaunitre. E. CHnsorasta

b" Front largement jaune soufré. E. Chaldon'ista

\section{Genre PIPIRIDEA}

a Moustaches noires sur les côtés de la grorge.

b) Point de moustaches noires.

P. castanementmis

P. venezullensis 


\section{FAMILLE TANAGRINAE}

A Bec fin, peu dilaté à la base, comprimé dans la moitié terminale.

$A^{\prime}$ Deuxième, troisième et quatrième rémiges les plus longues et égales; narines couvertes en grande partie de plumules frontales; couleur prédominante bleu foncé.

TaNagrella

A" Deuxième et troisième rémiges les plus longues et presque égales; narines presque rondes et découvertes; couleur prédominante vert brillant; plumes de la tache parotique à barbules jointes en faisceaux rappelant une production de cire.

Chlorochrisi

B Bec court, peu épais, élargi à la base; queue médiocre; rectrices presque égales entre elles.

B' Deuxième, troisième et quatrième rémiges les plus longues, première = cinquième; couleurs en général vives, à éclat vitreux, changeant de nuance vers la lumière.

Catliste

b' Bec plus élargi à la base; couleur dominante bleue.

\section{DirA}

C Bec court et épais.

C' Narines couvertes par les plumes frontales; bec comprimé, plus haut que large à la hase; quatrième rémige la plus longue.

IRIDORNAs

$C^{\prime \prime}$ Narines rondes, ouvertes en entier.

CA Bec aussi haut que large à la base; deuxiène et troisième rémiges les plus longues, première plus courte que la quatrième.

TAMAGRA

$C B$ Bec plus haut que large à la base; troisiène rémige la plus longue; taille forte.

Butmriupis

$C C$ Bec à arète dorsale peu courbéé; narines oblongues.

$\mathrm{Ca}$ Une tache temporale jaune ou rouge, correspondante à la couleur de l'abdomen; troisième et quatrième rémiges les plus longues; première $=$ septième.

Cb Une grosse tache jaune cervico-nucale; troisième et quatrième rémiges les plus longues; première = sixième.

Compsogona

D Bec à mandibule fort élevée, plate, à base arrondic, élevée au-dessus du plumage voisin, d'une couleur' plombée. 
E Bec plus long que la moitié de la tète, à ligne dorsale faiblement courbe, termince en crochet courbé, plus haut que large à la base.

E' Bord de la mâchoire droit non armé d'une dent médiane.

E.1 Cils maxillaires fort développés; coloration semblable dans les deux sexes.

TrighothraUpis

EB Cils maxillaires peu déreloppés.

Ea Plumage dissemblable dans les sexes, beaucoup de noir chez les mâles.

Tachiphones

Eb Plumage semblable dans les deux sexes; rien de noir; une luppe cervicale plate plus ou moins développée.

Euconetis

E* Bord de la màchoire armé au milicu d'une dent plus ou moins déreloppée.

$E A$ Bec plus ou moins faible.

Ea Tète noire uniforme.

LaNio

Eb Tìte à bande médiane largement rousse. Creurgors

$E B$ Bec épais.

Ea Quatrième et cinquième rémiges les plus longues; première $=$ huitième.

PHOLNGOTHR.ALPL

Eb Deuxième jusqu'à la cinquième rémige les plus longues; première plus longue ou égale à la sixième.

Priangid

F Bec plus ou moins faible, plus haut que large ì la base, droit. à ligne dorsale peu courbée; coloration des deux sexes semblable ou peu différente.

F' Première rémige égale à la neuvième.

Nevosta

f.' Première rémige plus longue que la liuitième, couleur prédominante olive.

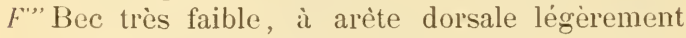
courbe, doigt externe sans ongle égal à l'interne.

Migrospingt's

\section{GENRE TAMAGRELLI}

a Front d'un bleu concolore au-dessous du corpes. T. mmisi

$b$ Front d'un céladon doré.

T. GALLOPHRIS

\section{Gexhe CALLISTE}

a Dos noir velouté, tête verte, dessous bleu de ciel, à gorge violette.
a' Croupion rouge feu.
C. IENI 
$a$ " Croupion jaune et rouge.

C. COELICOLOR

$b$ Dos noir mat.

b' Tête avec la gorge el le cou bleus.

C. GTANILULLis

$b$ " Tète et cou lilacés, gorge et joues vert pomme. C. Nigricincta

b' Tète noire, it sourcils trés larges et gorge verts.

C. MELANOTIS

b.", Tète à sommet vert, dessous du corps tacheté de rert et de noir.

C. Blarlepscit

$b$ "'," Tète à sommet et la nuque jaune, front et joues d'un rouge miniacé.

C. Parzedaki

c Dos noir squamulé de vert.

c' Milieu du ventre jaune.

C. Xanthogastri

c." Milieu du ventre blanc.

C. pungtata

il Dos vert strié de noir.

$d$ Croupion jaune.

$d^{\text {a }}$ Cótés de la tète noirs, avec la gorge et une

tache antéoculaire vertes.

C. Schranki

d" Croupion vert, tête jaune, gorge noire.

$d^{\mathrm{b}}$ 'Tête d'un jaune doré en entier.

C. venusta

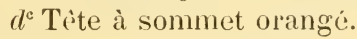

C. XaNthocephala

d"' Croupion bleu verdâtre.

$d^{\mathrm{e}}$ Front jaune doré, tache auriculaire orangé doré, bordée en dessous de noir.

C. chrysotis

e Dos straminé, sommet de la tète roux, la gorge et le devant du cou d'un bleu violâtre.

C. GYANOL.ENA

f Dos noir strié de jaune, la tète et le dessous du corps jaune, gorge marron foncé.

C. pulchra

f) Dos rert, dessous bleu, tite marron rougeâtre.

ん Plumage général bleu, dos noir, milieu de l'abdomen jaune sulfureux.

C. GYROLOÏDES

Plumage général bleu céladon; nuque traversée d'une raie d'un roux soyeux peu large.

C. BOLIVlaNA

- Sommet de la tite et dessous du corps largement noirs.

l' Dos bleu argenté.

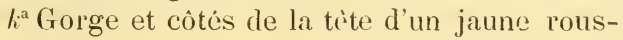
sìtre.

C. ARgentea

$k^{\text {b }}$ Gorge et còtés de la tite d'un rert pomme jaunâtre.

C. Viridicoleis

l. Dos straminé argenté.

$k^{c}$ Gorge et côtés de la tète rert céladon.

C. Argrophenges

\section{Genre DIVA}

a Plumage général bleu.

D. VAssori

b Plumage général bleu, à tìte céladon grisâtre.

D. Brantckll 
c Plumage général bleu, à dos noir, tache nucale d'un blanc stramine.

D. ATROC.ERULEA

\section{GENE IRIDORNIS}

a Tète jaune au sommet, dos bleu, abdomen roux. I. JeLskil

$b$ Trete noire, demi-collier nucal jaune, dos et poitrine bleus, ventre verdâtre sale.

I. Rieinhardti

c Tête à sommet ardoisé foncé, dos olive, gorge jaune, abdomen ocreux.

I. IXALIS

\section{Gexre TMNagIRI}

a Plumage général cendré bleuâtre.

a' Petites tectrices alaires d'un blanc violàtre. T. roelestıs

a" Petites tectrices alaires d'un bleu outremer, le cendré du corps coloré plus ou inoins de verdàtre.

T. C.INA

b Plumage général olive violàtre; tête, devant de l'aile vert ponme jaunâtre.

T. MELAMOPTERA

c Dessous du corps jaune.

c' D'une nuance sulfurée; dos olive brunâtre; la tête avec le cou et les ailes bleus.

T. DARWIVI

c" Poitrine orangéc; dos noir; la tête avec le cou et les ailes bleus.

T. STRIATA

d Dessous du corps cendré ardoisé, dos vert oliri, sommet de la tite bleu outremer.

T. CXANOCLPIILA

\section{Gexhe P(ECILATHIR U PIS}

a Dessous du corps jaune.

$a^{\prime}$ Tête et dos bleuâtre, tache lacrymale jaune. P. LAckryos

a" Tète et dos ardoisé olivàtre, à peine bleuâtre, taches lacrymales et palpébrales jaunes. P. p.l一pernosa

$b$ Poitrine et abdomen d'un rouge vif.

b' Sous-caudales rouges variées de noir. P. Igricrissı

$b$ " Sous-caudales rouges en entier. P. igniventris

\section{GENRE RHAMPIOCELUS}

a Dos, ailes el queue noirs; la tète, le cou et le dessous rouges, masque céphalique et milieu du ventre noirs.

R. NIGROGULARIS

b Plumage général noir rougeâtre, à croupion et les sous-caudales rouges.

R. IHCHAN 
c Tète et cou d'un rouge obscur.

c' Abdomen et dos d'un rouge très obscur. R. JACAPA

(" Abdomen et dos noirs.

R. ATROSERICEIS

\section{Gevie 'TACHYPHONUS}

a Plumage général du mile noir.

a' Une tache humérale blanche.

'T. MELALEUCOS

a" Bande humérale rouge.

T. PHOENIGEIS

a" Croupion fauve.

aa Le noir du plumage général coracin, milieu du sommet de la tète faure, còtés du bas-ventre roux.

'T. NAPENSIS

ab Le noir du plumage mat.

$a_{\propto}$. Gorge fauve, milieu du sommet de la tìte orangé rougeâtre, à bordures fauves.

T. GRISTATUS

$a \beta$ Poitrine et ventre roux, tache verticale fauve.

T. RUFIVENTRIS

\section{GENRE PHIANICO'TIIR I CPIS}

a liouge sale, à huppe interne rouge feu.

Ph. peruvianus

b) Olire jaunâtre, à gorge striée de jaune.

Ph. Carmioli

\section{Gexre Pyrayga}

$a$ Mâle tout rouge.

$a^{\prime}$ D'un rouge rosé; bec non denté. P. estrva

a" D'un rouge cinabarin; bec denté. P. Azarde

$b$ Rouge à ailes et queue noires, deux bandes blanches en travers de l'aile. P. ARDExs

c Rouge sur la tête, le cou et la poitrine, jaune sur l'abdomen et le croupion.

P. rubriceps

\section{GeNRe NEYIOSIA}

a Grise en dessus.

$a$ ' Tête rousse en entier.

aa Dessous du corps roux, à milieu du ventre

blanc.

N. ornata

ab Gorge et poitrine rousses, abdomen blanc, à flancs gris.

N. PECTORALIS

$a$ "Sommet de la tète gris, côtés de la tête roussitres. 
aa Gorge jaunâtre, dessous roussâtre, à milieu du ventre blanc.

N. SORDIDA

ab La gorge et tout le dessous ocrens.

N. INORNATA

$b$ Vert olive en dessus, còtés de la tète et gorge noirs.

$b$ ' Sourcils et demi-collier d'un jaune sulfureux. N. gurra

$b$ " Sourcil roux foncé très fin.

N. GUIRINA

c Dos noir sur les côtès, jaune au milieu; gorge jaune, le reste du dessous blanc.

$c$ Point de jaune sur l'aile.

N. Flavicollis

c" Tache jaune sur l'aile.

d Dessus du corps bleuâtre, dessous blanc.

N. peruana

N. PILEATA

\section{Genre Clitorosipingus}

a Olive verdàtre en dessus.

a' Olive jaunâtre en dessous. milieu de la gorge jaunâtre; sourcil jaunâtre peu marqué.

Ch. oleagineus

a" Blanc grisâtre en dessous, à flancs olives.

aa Tête schistacé brunâtre, gorge et devant du cou fauve blanchattre.

Ch. cinereocephales

$a b$ Tête brun noirâtre, à grosse tache postoculaire blanche, bande pectorale olive paile. Ch. albitemporatis

$a$ "' Gris sale en dessous, tête concolore au dos, gorge jaune.

Ch. Flavigularis

a"' Jaune en dessous.

aa Tête noire au sommet et les côtés, sourcils blancs très longs.

Ch. aubicularis

$a b$ Tête cendré ardoisé, gorge et poitrine cendré blanchâtre; sous-caudales roussâtres.

Cit. Ghrisogaster

ac Front jaune, sourcils jaunes verdâtros; sous-caudales roussâtres.

Ch. Frontalis

ad Front cendré, sourcils blancs.

CH. superciliosus

b Gris en dessus.

b' Blanchâtre en dessous.

ba Sourcils et tache génale blancs.

Chi. leucogaster

b) Point de sourcils blancs, bec mince.

Ch. мinthophthalues:

b)" Roux en dessous.

ba 'Tète et gorge noires, sourcils blancs longs et larges.

Ch. castaneicollis

bb Tête cendrée au sommet, noire sur les côtés.

Cit. Berlepschi

\section{TRIBU ARREMONIN $\mathbb{E}$}

A Queue plus longue que l'aile.

A' Queue fort étagée, plumage blanc et noir. Cissoprs 
1" Couleur du dessous jaune, blanche ou cendrée;

sommet do la tete roux; bec rourt.

Cakmoghmolis

B (Surue moins longue que l'aile.

l:' Plumage général vert rif. P'sirtosipls

l'" Coloration modeste.

B. I Bec conique, à mâchoire courbée légèrement au bout.

$\mathrm{Ba}$ Aile plus longue que la queue.

Arremon

$\mathrm{Bb}$ Aile aussi longue que la queue; bec inćdiocre; pattes fortes.

Buarremon

$B B$ Bec robuste, élevé.

Ba Bords de la mâchoire droits jusqu'à l'extrémité courbée, non armés d'une dent.

Ba Taille forte, quatrième rémige la plus longue.

SALTATOR

$B \beta$ Taille médiocre, troisième rémige la plus longue.

Orchesticus

Bb Bords de la mâchoire découpés au milieu en une dent.

Ba Taille forte; dent forte; échancrure terminale bien prononcée.

\section{Pitylus}

$B \beta$ Taille médiocre; dent faible; échancrure terminale presque nulle.

Conothraupis

\section{Gente Carenochirous}

a Dessous du corps jaune.

$a^{\prime}$ Gorge jaune.

a Miroir alaire blanc, dos et queue ardoisés; sommet de la tite et nuque largement roux.

C. Latingents

ab Miroir alaire nul; dos et queue olive sale, milieu de la tète et de la nuque d'un jaune sale.
C. TRICOLOR

a" Gorge noire; dos fuligineux noirâtre, aile sans miroir; somınet de la tête et nuque largement roux vif.

C. MELANOL. EMUS

b) Dessous du corps blanc ou blanc roussâtre.

b' Miroir alaire blanc volumineux; front longuement noir, bordé des deux côtés de blanc, grosse tache cervicale fauve.

C. Dresseri

b" Miroir alaire nul, front longuement noir, bordé des deux côtés de blanc, le reste du sommet de la tète marron foncé.

C. SеebuhM

c Dessous du corps cendré plombé, gorge large- 
ment blanche, à longues moustaches noires; milieu du front noir, còtés blancs; sommet de la tête et nuque roux; miroir nul.

G. Taczanowski

\section{GENRE ARREMON}

a Dos olive brunàtre; sommet de la tête noir, à raie médiane cendrée; devant de l'aile orangé; bec rouge.

A. ERYTHRORHYNCHUS

b Dos olive verdâtre, sommet de la tête tout noir; bec noir.

A. NIGRICEPS

c Dos ardoisé; sommet de la tête tout noir; bec noir.

A. Abeillei

\section{GENRE BU IRIREMON}

$a$ Olive en dessus.

$a^{\prime}$ Sommet de la tête roux, demi-collier noir.

B. BRUNALINUGHUS

a" Sommet de la tête cendré au milieu, noir sur les côtés.

aa Demi-collier noir.

B. Torquatus

ab Point de demi-collier noil.

B. AFFINIS

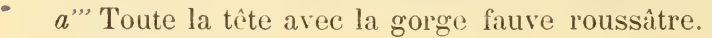

B. Fulviceps

b Ardoisé en dessus.

b' 'Toute la tête avec la gorge blanche et une grosse tache noire cervicale.

B. ALBICEPS

b" Tête noirâtre, gorge largement avec le bas des joues d'un blanc isabelle, moustaches noires courtes.

B. Nationi

\section{Gevre SALT'TOR}

a Dos plus ou moins olive.

a' Sourcils blancs.

$a^{a}$ Gorge blanche par-devant, ocreuse en bas, bordée des deux côtés d'une large moustache noire.

S. MAGNLS

$a^{\text {b }}$ Sourcil fin, gorge blanche bordée des deux côtés d'une moustache grise foncé. \$. superciliaris

$a^{e}$ Sourcil très large lavé de jaunâtre, gorge blanche sans moustaches, poitrine enduite de jaune.

S. FLAVIDICOLLIS

a" Point de sourcil blanc, milieu de la gorge blanc bordé largement de gris olivattre, poitrine et abdomen fortement stries. 
$b$ Dos plombé.

b' Gorge blanche bordée de moustaches noires, poitrine et abdomen gris cendré, queue unicolore.

S. carulescens

li" Gorge fauve blanchâtre bordée de moustaches noires, poitrine et abdomen fauve grisaitre. queue unicolore.

S. Azare

b"' Grorge blanche au milieu mème, còtés de la tìte, de la gorge et le devant du cou noirs, poitrine cendrée ou fauve grisâtre, du blanc à l'extrémité des deux rectrices externes.

S. Latiglavius

\section{FAMILLE FRINGILLIDÆ}

A Bec épais.

A' Ligne dorsale du bec arquée.

A 4 Ligne dorsale du bec fort arquée, narines latérales.

Neorhysches

$A B$ Dos du bec bossu à la base, à courbure très faible après avoir dépassé les narines; narines voisines du soinmet.

Gnathospiza

$A C$ Ligne dorsale du bec en arc faible. Aa Narines voisines du sommet.

A \%. Extrémité de la màchoire courbée en bas, à échancrure profonde; couleur générale jaune avec du noir.

Pheuctiges

A $\beta$ Extrémité de la màchoire à peine inclinée, à échancrure faible; couleur générale bleue chez les mâles. Guiracı

$A_{\eta}$ Bec bossu à la base, puis abaissé en ligne droite.

$A b$ Narines petites situées dans la moitié Sporophila de la hauteur de la mâchoire; courbure du dos médiocre.

\section{Piezorhina}

A" Bec très court.

AA Pyrrhulacé, à dos en courbe régulière. Spermophlla

$A B$ Bec bossu à la base, puis à courbe trìs

faible.

Catamenia

$A C$ Bec comprimé, à arète dorsale largement aplatie, dans toute sa longueur, les côtés creusés.

Catamblyahychus

B Bec conique.

B' Queue fort arrondie à l'extrémité.

$B A$ Plumage du dos strié de foncé sur un fond clair. 
Ba Bec plus haut que large, comprimé sur les côtés.

HeNOPHILA

Bb Bec presque aussi large que haut.

Coturniculus

$B B$ Plumage noir métallique.

Ba Noir en entier.

Volatinia

Bb Blanc en dessous, tète rouge.

Paroaria

BC Plumage cendré uniforme; bec sylviforme. Xexosprats

I)" Queuc peu arrondie à l'extrémité.

BA Queue unicolore; huppe rouge chez les mâles.

CORYPHOSPINGits

BB Barbe interne des rectrices blanche. Poospiza

B"' Queue légèrement entaillée.

BA Troisième rémige la plus longue.

Ba Aucune trace d'échancrure maxillaire, extrémité de la mâchoire non courbée. Duuca

Bb Extrémité de la mâchoire courbée légèrement, quelquefois une trace de l"échancrure.

Phrygilus

lic Ailes courtes.

ZoNotrichia

BB Deuxieme et troisieme remiges les plus longues.

Ba Queue jaune à la base, ou blanche en partie.

Bb Queue unicolore.

Chrysonitris

Sycalis

C Queue à rectrices presque égales, bec semblable à ceux des Conirostres, mais un peu plus gros, à dos légèrement enfoncé devant les narines.

\section{Sponiornis}

\section{Gexke PIIEUC'TICUS}

a La tite, le cou et tout le dessus noirs.

$a^{\prime}$ Croupion noir.

Ph. aureiventris

$a^{\prime \prime}$ Croupion jaune.

Ph. uropygialis

b Ia teite, le cou et tout le dessous jaunes.

PH. chrysogaster

\section{GENRE SPERMOPIILA}

a Noir en dessus et sur la tète, dessous blanc.

$a$ ' 'Tout le cou avec la gorge, la poitrine et les cotés de l'abdomen blanes, le miroir alaire b]anc.

S. hreteros

a" Milieu de la gorge et de la poitrine noir bordé de blane, plumes des flancs ocellées, miroir alaire blanc, petit.

A. OCELTATA

i Dos cendré.

b' Gorge, milieu de la poitrine et une raie le long du milieu de l'abtomen roux.

N. CASTANEIVENTRIS 
b" Dos strié de noirâtre; gorge marron foncé, reste du dessous blanc.

S. TELASCO

c Gris olivâtre en dessus.

c' Le front, les joues, la gorge et le haut de la poitrine noirâtres; abdonen jaune soufré pâle.

A. GLTTURILI

c" Dessous du corps blanc jaunàtre, miroir alaire et deux bandes transalaires blancs.

S. SIIPLEX

c" Dessous du corps gris foncé.

S. OBSGUBA

."” Dessous du corps gris pâle.

S. PAUPER

\section{Gente Catahenia}

a (queue traversée d'une large bande blanche.

$a^{\prime}$ Plus fort; bordures blanches aux réniges primaires non dilatees à la base pour former un miroir alaire, très peu de blanchàtre au milieu du bas-ventre.

C. ANAILS

$a^{\prime \prime}$ Moins fort; bordures blanches aux rémiges primaires dilatées à la base en formant un miroir alaire, milieu du ventre blanc.

C. AN.LOÏLEs

$b$ Queue sans bande blanche.
b' Dos strić de noir.
C. hufirostris
$b$ " Dos non strić.
C. Hovochro.

\section{GENRE CORY PIIOSPINGUS}

a Noir en dessus, ì liuppe et le dessous rouge intense.

C. chuenta

$b$ Dos gris brunâtre lavé de rouge, huppe d'un rouge vif, dessous du corps rouge carminé.

C. CRISTATA

$c$ Dos cendré ardoisé, huppe rouge intense bordée de noir, dessous cendré clair, à milieu blanc.

C. PILELTtS

\section{Gevie PoOspiza}

a Cendré bleuâtre en dessus, à dos lavé de faure, tache pectorale noire, barbe interne des rectrices en grande partie blanche.

P. BONAPARTEI

$b$ Ardoisé brunatre en dessus, la poitrine et le haut des cotés de l'abdomen roux.

P. L. ESAR

\section{GeNRe PIIRYgILUS}

a La tête avec la gorge et le cou supérieur cendré plombé. 
$a^{\prime}$ Dos olive verdâtre, dessous du corps jaune sans nuance rousse.

$a^{\prime \prime}$ Dos olive jaunâtre et dessous du corps jaune avec une nuance rousse.

$b$ La tète arec la gorge et le cou supérieur noir ou noiràtre, dos roux, ventre jaune au milieu, poitrine et flanes roux.

Plumage général gris ou cendré.

c' Queue traversće d'une large bande blanche. Ph. alaudines

c" Queue sans bande.

$c^{2}$ La gorge, le cou antérieur et le milieu de la poitrine et de l'abdomen noir's squamulés de blanc.

PH. Fruticeti

$c^{\text {b }}$ 'Tout le corps cendré bleuâtre jlus clair en dessous qu'en dessus.

$c^{c}$ Dessus du corps gris strié de noir, dessous du corps et bande sourcilière gris blanchâtre.

$c \alpha$ Longueur de l'aile, 71-76 millimètres. Ph. Plebejts $c \beta$ Longueur de l'aile, 60-65 millimètres. Ph. oguianis

\section{GENRE CHRYSOMI'TRIS}

a Plumage général noir, à bande transalaire et la région anale jaunes.

CH. ATR.IT.I

b) 'Tout le dessus noir, tout le dessous jaune, miroir' alaire blanc.

Cils. goldimbanis

c Tète noire, dos olive.

c' Croupion et le dessous du corys jaune sulfureux. Ch. Caprtalis $c$ "Croupion et le dessous du corps jaune orangé. Ch. Sieniranzkil

d La tète et tout le cou antérieur noirs, dos noirâtre frangé d'olive, le croupion, le dessous du corps et une bande transalaire jaune sulfureux. Ch. troprgilsts

\section{GENRE SYCALIS}

$a$ Olive jaunåtre en dessus, sommet de la tête orangé.

$b$ Tout le corps jaune tirant au verdàtre en dessus. S. Letea

$c$ Dos gris verdâtre, somınet de la tête olive jaunâtre, croupion jaune verdâtre uniforme.

S. CHLORis

d Dos cendré, tète jaune, croupion olive jaunâtre. S. cropralats

$e$ Dos gris strié de noirâtre.

e' Dessous du corps arec les flancs jaunes.

A.. LUTEITENTRIS

c" Dessous du corps jaune à flancs gris.

S. ARVENSIS

f Dos roux, dessous cendré.

S. ERTTHRONuTA 


\section{FaMILLE PICIDA}

A Queue courte, à rectrices non raides, arrondie au bout.

Pigcinges

B Queue fort étagée, à rectrices raides, les médianes les plus longues, fort atténuées à l'extrémité, à baguette épaisse.

B' Sillons sus-nasaux saillants, prolongés jusque pries du bout de la màchoire.

$B A$ Bec droit, robuste; queue longue; plumage général noir et rouge.

Ba Doigt antérieur externe plus long que le postérieur externe.

DrYOCOPLS

$\mathrm{Bb}$ Doigt antérieur externe moins long que le postérieur externe.

Chapephilus

BB Bec moins robuste; queue médiocre; ailes rayées en traver's de blanc ou dé blanchàtre. Picus

I'" Sillons sus-nasaux moins saillants et moins prolongés.

B.A Ailes non rayées en travers, rémiges à baguette brune.

Chloronerpes

$B B$ Ailes rayées en travers, rémiges à baguette jaune.

Chrysoptilus

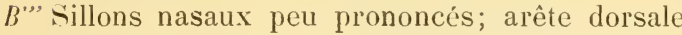

fort arquée.

Geleus

I)"' Sillons nasaux presque nuls ou peu marqués.

B.A Arête dorsale du bec saillante.

HYpoxanthes

$B B$ Arète dorsale du bec arrondie peu saillante.

$B a$ Bec moins long que la tète.

$\mathrm{Bb}$ Bec plus long que la tête.

Melanerpe:

Colaptes

\section{Gexne PiCUnNes}

a Dessous du corps immaculé.

$a^{\prime}$ Dessous d'un cannelle foncé.

P. RUFinentris

$a^{\prime \prime}$ Dessous roussàtre.

P. Castelnaud

$b$ Dessous du corps rayé ou maculé.

$b$. Dessus varié de macul's bien distinctes.

$b^{\text {a }}$ Dessous jaunâtre rayé sur toute la surface de brun noiràtre.

P. BuFfoNI

b" Dessus à bordures claires dans les plumes, peu prononcées.

$b^{\text {b }}$ Dessous jaunâtre rayé en traver's sur toute 
la surface de brun noirâtre, front du mâle ponctué de jaune doré.

P. punctifrons

$l^{\circ}$ Dessous blanchatre, à gorge et poitrine rayés en traver's de noir, l'abdomen longitudinalement de noir, front du mâle parsemé de glos points blancs à la base et jaunes au sommet.

\section{P. SGlateri}

$b^{\mathrm{a}}$ Poitrine noire maculée de blanc, abdomen rayé en traver's de noir et de blanc, front strié longuement de rouge cinabarin.

$b^{\mathrm{e}}$ Poitrine blanche rayée en travers de noir, abdomen blanchâtre maculé de noir, front du mâle strié de rouge.

\section{P. Steindahaneri}

P. JELSKII

\section{Gente CAMPEPHILUS}

a Poitrine et abdomen rayés en travers de noir sur un fond blanc roussâtre.
a' Croupion tout noir.
C. melanoleugus
a" Croupion rayé de fauve.
C. Sclateri

b) Dessous du corps non rayé.

b' Poitrine et abdomen d'un roux cannelle, enduit de rouge sur le devant, barbe externe des rémiges primaires cannelle en partie.

b" Poitrine, abdomen et croupion rouge sang.
C. TRAGHELOPYRU:
C. HeMnTOGASTER

\section{Genre Picus}

a Dessous non maculé, gorge et cou antérieur jaune, front longuement blanc.

P. Cactorum

$b$ Dessous maculé de brun, front concolore au sommet de la tète.

P. LigMarius

\section{Genre CIlloronerpes}

(1) Tout le corps presque unicolore sans taches ni raies.

b Dessus rouge, dessous blanc ondulé de gris, còtés de la tète blanchatres avec tache brune auriculaire.

Ch. fumgates

Dessus du corps olive.

Ch. Callonotus peruviANUS

$c$ ' Dessous rayé en traver's de brun.

$c^{\text {a }}$ Des raies sur tout le dessous jusıu'ì l'extrémité des sous-caudales, du rouge sur les tectrices alaires.

Ch. HILARIS. 
$c^{b}$ Le ventre et les sous-caudales non rayés, milieu du front et du vertex ordinairement sans rouge.

Ch. canipileus

c" Dessous à grosses macules claires sur la poitrine et des raies transversales foncées sur le ventre et les sous-caudales.

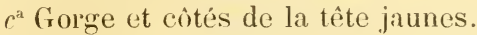

Ch. rhaviliulabis

$c^{\mathrm{b}}$ Gorge blanchâtre, côtés de la tète olive jaunâtre, avec une raie jaune orangée audessus du rouge malaire.

Ch. leugolames

\section{Gente CHRYsOp'THLS}

a Gorge et devant du cou noirs.

Ch. atricollis

$b$ Gorge noire tachetće de blanc, poitrine ponctuée de noir.

Ch. punctipegtus

\section{Genre COLAP'TES}

a Région jugulaire variée de lignes transversales noires.

C. STOLzuinni

$b$ Région jugulaire variée de taches cordiformes noires.

C. PUNA

\section{Genre CeLEUS}

a Plumage du corps rayé de noir sur "un fond

- roux brunâtre.

$a$ ' Le devant du cou et la poitrine noirs. C. Tinnexculus

$a$ " La gorge et le devant du cou de la couleur uniforme tachetée de noiràtre.

C. GRAMUICUS

$b$ Plumage du corps uniforme sans taches.

b' Roux foncé, à croupion et les flancs de l'abdomen jaune sale.

C. JUMANA

b" Roux rougeâtre très foncé, croupion jaune citron roussâtre.

C. CITREOPYGIL'S

b" Plumage du corps jaune peu intense.

C. citrinus 


\section{FAMILLE ALCEDINIDÆ}

Bec long, comprimé, assez élevé, à arète dorsale aplatie en dessus et pénétrant profondément entre les plumes frontales; queue longue, large, à rectrices externes considérablement plus courtes, les autres égales.

Ceryle

\section{GENRE CEIRYLE}

a Couleur du dessus non métallique.

Dos cendré bleuâtre tacheté de blanc. C. Torquata

$b$ Couleur du dessus vert foncé métallique.

b) Dessous roux ell entier, une bande pectorale verte chez le ox.

C. INDA

b" Dessous roux, à milieu de l'abdomen blanc, bande pectorale verte chez la $Q$.

C. superciliosis

b" Dessous blanc, à bande pectorale rousse chez le $\sigma^{x}$, aile non maculée de blanc ou à peine. C. anazova

b)" "Dessous blanc, à bande pectorale rousse chez le $\sigma^{7}$, verte chez la $Q$, aile plus ou moins maculée de blanc.

C. C.IBANisi

b" "' Dessous blanc, bande pectorale large rousse chez le ơ, deux vertes chez la $Q$, tectrices alaires non maculées de blanc.

C. anerigana

\section{FAMILLE MOMOTIDAE}

A Bec ordinaire non dilaté.

A' La partie antéapicale de la baguctte des deux rectrices médianes dénudée.

$a^{\text {a }}$ Couronne céphalique bleue.

Monotus

$a^{\text {b }}$ Point de couronne céphalique bleue.

Urospatha

A" Rectrices médianes barbées en entier.

BARTPHTHENGUS

B Bec dilaté et aplati.

Prionimiments

\section{Genke Mono'TL}

a Toute la partic postoculaire de la couronne céphalique saphirée, du roux sur la nuque.

M. BR.ASILIEXSIS 
$b$ Partie postérieure de la couronne céphalique concolore à la frontale avec une bordure saphirée, point de roux à la nuque.

b' Une bordure bleue sous le noir des joues. M. Mirrostephands

b" Point de bordure bleue sous le noir des joues. M. EQuatorialis

\section{FANILLE GALBULID盾}

A Bec fin, presque droit, graduellement rétréci vers l'extrémité.

A' Queue médiocre graduée.

Galbula

A" Queue longue fort étagée, à rectrices médianes très longues et atténuées.

UROGALBA

A"'Queve coupée carrément.

BRAGHYGALBA

B Bec élargi, à extrémité fort atténuée, l'arête dorsale arquée.

JAGAMEROPS

C Bec fort comprimé, élevé, à arète dorsale tranchante.

Galbagirhynchus

\section{Genke Ga bubua}

a"Bec tout noir.

a' Vert doré en dessus, à sommet de la tète vert bleuâtre, gorge et poitrine vertes.

G. TOMB.ACE. 1

a" Rouge en dessus et sur la poitrine, sommet de la tète vert bronzé.

G. ertthrothorax

$b$ Bec jaune, à moitié terminale de la mâchoire noire. b' Sommet de la tète pourpré très obscur passant au violàtre dans certaines directions de la lumière.

G. albirostris

b" Sommet de la tête noir rougcâtre tirant au bleu sous certain jour.

G. chalcogephala

\section{GENRE BRACHYGALBA}

a Gorge concolore à la poitrine, à peine striée de blanchâtre.

B. INORNATA

$b$ Gorge blanche.

B. ALBIGULARIS 


\section{FAMILLE BUCCONIDE}

A Queue dépassant beaucoup le bout des ailes.

L' Bec droit, voùté, à dos de la mîchoire courbé fortement dans sa partie terminale, dessous de la mandibule arqué ver's le haut.

.1" Bec comprimé non voùté, droit, courbé à l'extrémité; l'extrémité de la mandibule courbée en bas.

.$^{\mathrm{a}}$ Plumes de la base de la mandibule allongées, lancéiformes, formant de chaque côté une moustache saillante.

.$^{\text {b }}$ Plumes de la gorge longues, à barbes désunies recourbées en avant; aile armée d'une courte épine cornée.

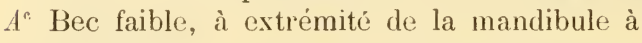
peine courbée, plumes dé la base de la mandibule criniformes recourbées sur les côtés. Nonvur.

B Ailes longues atteignant l'extrémité de la queue. Chemidopter.

\section{GeNhe BUCCO}

a Parties supérieures du corps noires.

$a$ ' Tète immaculée au sommet.

$a^{\mathrm{a}}$ Devant du front finement blanc, sous-

alaires noires.
$a^{b}$ Front longuement blanc, sous-alaires noires

B. MAGRORHYNGHUS traversées d'une raie oblique blanche. B. HYPERRHYTchls

$a$ " Tête maculée au sommet de blanc.

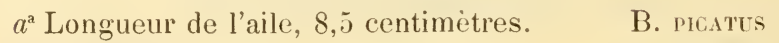

$a^{\mathrm{b}}$ Longueur de l'aile, 7 centimètres. B. coldaris

b. Parties supérieures du corps brunes, à sommet de la tête plus ou moins roussâtre.

b' Bec noir.

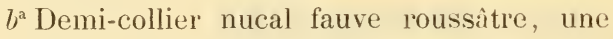
large bande noire au-dessous de la gorge. B. sacrodactruts

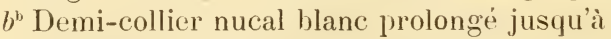
la naissance du bec, grosses taches noires sur la poitrine et l'abdomen.

B. pulientud

$b^{c}$ Dessous blanc crème varié de stries noires en forme de pinceau.

B. LANGLOLATUS

b" Bec jaune.

Collier nucal blanc, rectrices rayées en traver's de fauve.

B. GHACURU 


\section{GENRE MALICOP'TILI}

a Plumage du corps non strié ni tacheté.

Dessus du corps roux brunatre, tète gris plombé strié tris finement de blanc.

II. RLFA

1) Plumage du corps plus ou moins strié.

li Brun strié de fauve.

II. FISCA

li" Isa poitrine et le haut de l'abdomen noirs striés de blanc.

M. FUlvogularis

\section{GeNre MONAS I}

$a$ Bec jaunc.

M. FLAYIROSTLIS

$b$ Bec rouge.

b' Front blanc, menton blanc tres finement.

M. peruana

b" Front noir.

II. NigrifroNs

\section{GeNRE NONNULA}

a Sommet de la tète roux marron.

N. riffigapillat

$b$ Sommet de la tête concolore au dos, dessous roux, à inilieu du ventre ocreux.

N. Brungea

\section{Fanille CAPITONIDÆ}

\section{Genre Capíto}

Bec comprimé, dilaté à la base, ì dos élevé entre les narines, pointu à l'extrémité, mâchoire dépassant un peu l'extrémité de la mandibule, première rémige très courte.

a Dessus noir tacheté de jaune, dessous jaune.

a' Gorge orangée, sommet de la tête olive jaunâtre.

a" (iorge rouge orangée, front longuement lavé de rouge.

C. amazonicls

b Dessus brun olivâtre, sommet de la tête rouge 
vermillon, gorge largement jusqu'au milieu de la poitrine orangée.

C. AUROVIREAS

r Tert ou vert olive en dessus.

$r$ Demi-collier nucal jaune verdatre. C. adrantricolus

c" Demi-collier nucal bleu.

$c^{\text {a }}$ Bas des joues largement bleu.

C. versicoror

$c^{\mathrm{b}}$ Bas des joues jaune.

$c \propto$ Haut de la gorge rouge, bas de cette jartie bleu.

C. Glaucogclaris

$c \beta$ Grorge rouge sans rien de bleu.

C. Steeri

\section{FAMILLE RHAMPHASTIDE}

A Bec énorme, à narines percées dans la face postérieure de l'élévation basale, queue à rectrices égales.

Rhamphastos

B Narines basales percées au sommet du bec, quene étagée, à rectrices peu larges.

li' Bec long et gros, à sommet aplati à la base, narines percées sur la face dorsale du bec, queue assez longue.

Pteroglossus

1)" Bec et queue beaucoup moins longs, une grosse tache jaune sur les oreilles.

Stilenider.

l,"'Bec médiocre plus ou moins sillonné sur lis còtés, à narines basales situéc's plus bas que le sommet du bec, et ordinairement dans une fosse, couleur générale du plumage vert pré. Avtacorhamphus l)"' Bec médiocre et large comparativement, non sillonné, à base dorsale un peu élevée entre les narines.

Andgena

\section{GeNRE RHAMPIISTOS}

$a$ Sus-caudales jaunes.

$a^{\prime}$ Poitrine blanche, bec noir, à còtés convexes. RH. Cuvilri

$a^{\prime \prime}$ Poitrine blanche, bec noir, à côtés plats.

RH. Colmerets

a" Poitrine blanc jaunâtre bordée de rouge, bec

noir varié de jaune et de rouge.

RH. NiCA

b) Sus-caudales blanches, poitrine jaine soufre bordée en dessous de rouge, bec à moitié oblique jaune.

RH. TOCABD

c Sus-caudales rouges, poitrine orangée borkée de soufré, joues blanches, bec noir.

Rh. vitellanes 


\section{GeNRE P'TEROGLOSSUS}

$a$ Plumes du sommet de la tète frisées, transformées à l'extrémité en une lamelle cornée. PT. Beaunarnaisin

b Plumes du sommet de la tète normale.

b) Une large bande jugulaire rouge.

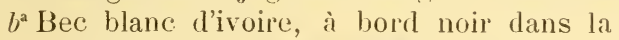
mâchoire.

Pt. flavirostris

$b^{b}$ Bec jaunâtre avec une raie gris olivâtre sur le bas des còtés de la mâchoire.

l, Une large bande rouge en travers de l'abdomen.

PT. Azar E

'Une large bande pectorale noire, l'abdominale rouge.

b"." Point de rouge en dessous.

Pt. castanotis

Pt. pluricinctus

Pt. Hunboldti

\section{Gente SELENIDER I}

a Bec noir, à base rougeâtre, flancs de l'abdomen safranés variés de rougeâtre.

b Bec noir, à base cornée, flanes de l'abdomen jaune orangé.

S. Reinivardti

S. Langsdorffi

$c$ Bec à mandibule olive à la base même, puis d'un blanc d'ivoire, à tache antéapicale noirâtre, l'extrémité blanc jaunâtre.

S. Gouldi

\section{Genre ANDigeya}

a Bec jaune à la base, avec une bande transversale noire, tout le dos et l'extrémité de la mâchoire rouge, l'extrémité de la mandibule noire. A. Hypoglaucus

$b$ Bec rouge à la base, noir au dos et à l'extrémité, avec une lamelle latérale blanc d'ivoire. A. Lamelurostris

\section{Genre IULACORIIMPIIUS}

a Du rouge au croupion.

a' Base du bec à bordure blanche.

a" Bec corné bleuatre à la base sans bordure basale.

A. H.EMATOPYGiUs

A. c.eruleicinctus

$b$ Croupion sans rouge.

b' Gorge noire, sous-caudales marron.

A. atrigularis

l)" Grorge blanche, sous-caudales vertes.

A. Derbiayes 


\section{F.MILLE TROGONIDAE}

A Front lisse, tectrices sus-caudales courtes.

'Trogion

B Front à plumes tournées vers le devant, formant souvent une crite plus ou moins développée, suscaudales fort prolongées.

Pilaronigrus

\section{Genre Trogon}

a Dessous du corps rouge.

a' Queue noire, à rectrices médianes vertes en dessus, externes mouchetées très faiblement de blanchâtre.

T. MELANURUS

$a "$ Queue rayée en travers de blanc sur les rectrices latérales.

$a^{a}$ Raies blanches de la queue et des tectrices alaires aussi larges que les noires.

'T. COLLARIS

$a^{\text {h}}$ Raies blanches fines sur la queue et les tectrices alaires.

ac. Le vert de la tète, de Ia poitrine et du croupion bleuâtre.

T. HELIOTHRIX

a $\beta$ Le vert de la tête et de la poitrine fort doré.

T. PROPINQUUS

ay Le vert du dos doré, le sommet de la tète et la poitrine d'un saphiré passant au violet.

T. VARIEGITUS

b) Dessous du corps jaune.

b' Dos vert, tête et poitrine saphirés.

$b^{\text {a }}$ Tectrices alaires d'un noir uniforme.

T. VIRIDIS

b) Tectrices alaires vermiculies finement de blanc.

T. RAMONIANS

l)" Dessus du corps vert.

$b$ a Sommet de la tète concolore au dos, poitrine verte.

T. ATRICOLLIS

$b^{\text {b }}$ Tète toute noire, poitrine saphirée.

'T. CALIGATUS

\section{GeNRe PIIARONACIRUS}

a Une longe crète élevée au front, deux rectrices de chaque côté blanches.

PH. Avtsingsis

$b$ Point de crète proéminente, rectrices latérales noires.

b) Les grandes sus-caudales dépassant l'extrémité des rectrices.

Pil. auricleps

l)" Les grandes sus-caudales n'atteignant pas l'extremité des rectrices. 


\section{PAWII.I. CUCULIDA}

A Bec tries haut, fort comprimé, à arite dor'sale fort élevée, les narines percées dans la moitié inférieure des faces latérales.

Crotophaga

B Bec fort, ailes courtes arrondies au bout, à septième, huitième et neuvième rémiges égales et les plus longues, tarse élevé.

Neomorphus

C Bec court ou médiocre légèrement arqué, à dos arrondi, tarse plus long que le doigt le plus long. c' Bec court comprimé, quatrième rémige la plus longue, dessus du corps tacheté.

Diplopterus

C" Bec médiocre, cinquiòme ou sixième rémige la plus longue, queue longue fort étagée, à rectrices larges.

C" Bec médiocre, troisième rémige la plus longue, queue longue, à rectrices peu larges.

Cocigyus

\section{Genre Cro'TOPHATA}

a Bec plus court que la tète.

a' Bec à côtés non sillonnés.

$a$ " Bec à côtés sillonnés.

C. sulcirostris

$b$ Bec plus long que la tête.

C. MAJOR

\section{Genre PIAYA}

$a$ Sommet de la tète cendré bleuàtre.

P. MELANOGASTRA

$b$ Sommet de la tête concolore au dos.

b' Abdomen cendré clair, bas-ventre noiràtre, taille forte.

P. CaYANa nigricrissa

b" Abdomen gris roussâtre, taille petite.

P. MINUTA

\section{Genre CocCYZus}

a Bande sous-oculaire noire.

G. milanoconyphus

$b$ Bande sous-oculaire nulle.

C. erithrophthalaies 


\section{FIMLLLE PSITTACID在}

A Queue étagée, à rectrices atténuées aiguës à l'extrémité.

A' Tour de l'oeil et joues dénudés, ailes moins longues que la quene.

A" Lores emplumés, tour de l'oeil dénudé, ailes aussi longues ou moins longues que la queue. Conures

A"'Toute la tête emplumée, deuxiène rémige la plus longue, troisieme à peine plus courte.

AA Queue plus courte que l'aile, bec comprimé. Brotogeris

Al) Queue un peu plus courte que l'aile, bec voùté, à dos arrondi.

Bolborhytehus

AC Queue faiblement arrondie, courte, mesurant à peine la moitié de la longueur de l'aile, à rectrices aiguës au bout.

Psittacula

B Queue courte, large, à rectrices plus ou moins arrondies à l'extrémité.

P' Bec gros, à dos un peu aplati à la base même et enfoncé en une gouttì̀re très courte, les rectrices subaiguës au bout.

Pachynus

B" Bec aussi élevé à la base que long, rectrices subaiguës à l'extrémité, les trois premières rémiges les plus longues.

UROGHRONA

$B$ "' Bec à dos aplati traversé par une longue gouttière.

BA Deuxième et troisieme, ou deuxième, troisième et quatrième rémiges les plus longues, sous-caudales rouges.

Piones

PB Dans l'aile deuxième et quatrième rémiges les plus longues, sous-caudales vertes.

$B C$ Queue un peu plus longue que la moitié de l'aile, sommet de la tête le plus souvent noir, sous-caudales jaunes ou vertes.

CaÏGA

\section{Genre ARA}

a Couleur principale rouge, grandes tectrices alaires jaunes.

1. Macao

$b$ Dessus bleu, dessous jaune.

A. ararauna

$c$ Couleur principale verte.

$\because$ Front rouge.

$c^{\text {a }}$ ''out le dessous vert uniforme.

A. Militaris 
$c^{b}$ Sommet de la tête vert bleuatiee, à front rouge brunatre foncé.

A. severa

c"Tout le sommet de la tite céladon grisàtre.

A. Coulowi

\section{Genre conurus}

a Couleur principale rerte.

a' Toute la tète verte, sous-alaires rouges. C. Guranexsis

a" Toute la tête brun grisattre enduite de bleu. C. Wendectu

a"' Du rouge sur la teite.

$a^{\text {a Front rouge. }}$

$a_{\%}$. Pli de l'aile rouge, grandes sous-alaires gris enduit de jaunàtre.

C. Frontates

aß Pli de l'aile vert, lores et tour de l'œil rouge.

C. mitratus

$a^{\mathrm{b}}$ Front et còtés de la tète rouges.

C. ervthrogenys

$b$ Queue d'un rouge obscur.

$b$ ' Tectrices du bord de l'aile d'un rouge vermillon.

$b^{2}$ Queue d'un rouge brun foncé en dessus.

$b^{\text {b }}$ Queue verte en dessus.

C. Souancei

C. RUpicola

b" Tectrices du bord de l'aile d'un rouge vermillon, à extrémités jaunes.

C. melanerus

b"' Tectrices du bord de l'aile vertes ou bleues.

$b^{\text {a }}$ Tache auriculaire jaune isabelle, devant du front rosé.

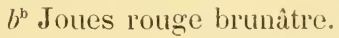

C. Rosethroys

C. Lectail

\section{GeNRE BRITTOGERYS}

a Grandes tectrices alaires vertes.

$a$ Còtés de la tète gris blanchatres, sous-alaires miniacées.

B. PYRRHOptera

a" Còtés de la tête verts, front jaunâtre, tache mentonnière orangée roussâtre.

B. JUGULARIS

a" Le devant de la tète et une strie sous-oculaire jaunes.

B. TUI

$b$ Quelques-unes des grandes tectrices alaires blanches ou jaunes.

b' Grandes tectrices secondaires jaunes, rémiges vertes.

B. Xavthoptera

6" Grandes tectrices secondaires jaunes, rémiges médianes blanches.

B. ViresceNs

\section{GeNRE BOLBORIIYNCIIUS}

a Devant du visage et dessous du corps plus ou moins jaune.

B. AURiFrons 
b Tout le plumage vert, queue peu étagée, à rectrices peu atténuées à l'extrémité.

B. ANDICOLA

\section{Genre PSit'Thcula}

$a$ Croupion vert, bec épais.

P. crassirostris

$b$ Croupion bleu.

b) Point de bleu sur la tête.

lia Dans les rémiges secondaires le bleu de ciel prolongé jusque près de l'extrémité des pennes.

P. PASAERINA

$b^{\text {b }}$ Dans les rémiges secondaires le bleu saphir s'arretant à une grande distance du bout des pennes.

b" Bande bleue postoculaire.

P. Sclateri

P. collestis

\section{Gevre PIONUS}

a 'Tète bleue.

P. menstrues

b) Tète d'un rouge sale, squamulé de foncé sur les ròtés.

P. Tumultuosus

\section{GeNre CIIBISOTIS}

$a$ Sommet et cotés de la tête verdàtres.

a' Point de rouge sur la barbe interne des reetrices, le vert comme poudré de farine, à bordures des plumes violâtres.

a" Du rouge sur les rectrices, bordures noiràtres sur les plumes du cou.

Cil. mercenalita

6 Croupion rouge, point de rouge sur les rémiges. Ch. Frstrya

$c$ Front bleu, còtés de la tète jaunes, du rouge aux rectrices.

d Tête verte arec une grosse tache jaune au milieu du sommet.

Cil. ughrocepHILA

\section{Gesre Ca ICA}

a Sommet d'un rouge brique, côtés de la tète, cou antérieur, flanes et sous-caudales jaunes.

C. X.NThomero-

$b$ Du noir sur la tête.

b' Toute la tête noire.

C. IIIsTRIO

b" Tite noire avec une grosse tache génale jaune. C. Barrabanit

b" Sommet de la tête noirâtre, unc ligne rerte au-dessous des yeux.

C. MELANOGEPIILAL 


\section{FIMILLE COLUMBID在}

A Premièe rémige non atténuée.

A' 'Tąrse court emplumé dans la moitié supéricure, denxieme et troisiene rémiges égales et les plus longues, queue ample arrondic.

Culumba

A" 'Taille petite, queue courte ar'ondie.

Aa Orbites dénuées.

Ia. Deuxième rémige la plus longue, queue non terminée de blanc.

Metriturelis

$A \beta$ Deuxième et troisiène rémiges les plus longues et égales, rectrice externe terminée longuement de blanc.

GrMNORELI.

Ab Orbites non dénuées, des bandes et des taches métalliques sur l'aile.

Chimapela

A" Ailes assez longues, queue plus ou moins allongée.

Aa Orbites dénuées, queue arrondie.

Melopelia

$A b$ Queue cunćiforme, à rectrices médiocrement étagées, deuxième rémige la plus longue.

1"' Tarse élevé, robuste, dénuć; ailes courtes, à deuxième, troisième et quatrième rémiges égales et les plus longues, queue courte.

Geothigun

B Première rémige atténuée dans sa partie terminale.

l'' Troisième et quatrième rémiges les plus longues, point de taches métalliques sur l'aile.

LEPTOPTILA

li" 'Troisième rémige la plus longue, des taches métalliques sur l'aile.

Peristera

\section{Gexpe COLUMBA}

a Tout le cou et le dessous du corps squamulé de foncé.

C. sreciosa

Cou non squamulé.

b' Demi-collier nucal blanc, cou postérieur métallique brillant.

C. ALBHINEA

b" Des taches fauves subarrondies au cou postérieur.

G. plumbea

b"' Point de taches ni bandes au cou postérieur.

$b^{\text {a }}$ Tectrices alaires bordées à l'extérieur de blanc.

C. AlBIPENNIS

$b^{\mathrm{b}}$ Dessus fuligineux foncé; tète, cou et dessous vincux.

C. VIXICEI 
$b^{c}$ Tout le plumage vineux, bec court.

C. subvinacea

$b^{\mathrm{u}}$ Dos roux rougeâtre, à éclat violet; le cervix, la nuque et le cou postérieur métallique brillant, gorge et joues cendrées.

C. RUFINA

\section{GENRE VETRIOPELI}

a Tectrices supérieures de la queue atteignant l'extrémité des rectrices, une tache métallique sur laile.

II. AYMARA

$b$ Tectrices sus-caudales beaucoup plus courtes que les rectrices, point de tache métallique sur l'aile.

M. MELANOPTERA

\section{GENRE CII I I NPELI}

a fous-alaires rousses.

a' Poitrine squamulée.

Ch. passerina

a" Poitrine non squanulée ni tachetée.

- Ch. griseola

$\dot{b}$ Sous-alaires internes noires, externes grises.

b' Dessus du corps gris.

$b^{a}$ Bande antérieure de l'aile rouge obscur, les autres taches alaires noir bleuàtre.

CH. CRUZiaxa

$b^{\text {b }}$ Toutes les taches alaires noir bleuatue.

Cil. Bugkletil

b" Plumage général cannelle rougeâtre.

Ch. Thlpacoti

\section{Genre PEIRISTERI}

a Rectrices externes blanches à l'extrémité.

a' Les deux bandes alaires postérieures marron violâtre.

P. Geofrroy

$a$ "'Toutes les trois bandes alaires saphirćes.

P. NONDETOURA

$b$ Rectrices noires jusru'à l'extrémité.

P. GINEREA

\section{GeNre LEP'TOP'TILI}

a 'lout le côté de la tète ocreux, une nuance vinacée assez forte sur la poitrine, éclat rouge violàtre au bas du cou postérieur.

L. RUFATILLA

$b$ Eclat vert tirant au bleuàtre au bas du cou postérieur.

L. OCHROPTERA

c Point d'ocreux sur les joues, la nuque étant d'une nuance rosée, point de vineux sur la poitrine. 


\section{GeNre GEO'TRYGON}

a Tout le dessus roux violet, poitrine vineuse.

G. gontani

b Dessus brun roussàtre, joures rosćes bordèes en d'ssous d'une ligne noire.

b' Sommot de la tête cendré bleuâtre.

G. Bovrcieli

b" Sommet de la tête gris lavé de rosé.

(i. FRENATA

\section{FAMILLE OPISTHOCOMIDE}

Bec épais, côtés de la tète dénués, plumes cervicales et nucales longues, linéaires, raides, légèrement courbées en avant formant une huppe élevée; queue longue arrondie.

\section{FaMILLE CRACIDÆ}

A Bec plus ou moins épais, le plus soment muni à la base d'un tubercule frontal.

.1' Oiseaux à tubercule frontal.

AA Tubercule médiocre couvert d'une cire, plumes du sommet de la tète et de la nuque élevées, à extrémité courbée en avant.

$A B$ Tubercule corné énorme, tête non liuppée. I'auxis

A" Point de tubercule fiontal.

1.4 Bec fort élevé, comprimé au sommot, tîte non huppée.

$A B$ Bec non élevé au sommet, une huppe de plumes allongées non recourbées disposées le long de tout le sommet de la tète.

B Bec médiocre plus allonge, sans tubercule frontal, gorge et devant du cou le plus souvent plus ou moins dénués.

b' Les côtès de la tête, la gorge et le haut du devant du cou dénués et parsencés de plumules criniformes rares; un pli cutané plus ou moins développè le long du milieu du cou.

B.A Plumes du milieu du sommet de la tète larges et arrondies. 
BB Plumes du sommet de la tête ailongées, presque linéaires, aiguës au bout, blanchâtres.

B" Gorge et devant du cou emplumés.

BA Les lores et les joues déplumés, point de fanon jugulaire.

Chamepetes

BB Tout le côté de la tête emplumé, excepté une bordure aur-dessous de l'œil, sur son devant et en arrière, un fanon cylindrique sur le milieu du cou supérieur.

B" Còtés de la tète et côtés de la gorge dénués, les plumes du cou supérieur aiguës ì l'extrémité.

\section{ORTALida}

\section{Genfe PENELOPE}

a Rémiges primaires blanches.

P. albipennis

b Rémiges primaires non blanches.

b' Tète sans stries blanches.

$b^{a}$ La peau des côtés de la tête parsemće de petites plumules; point de roux sur l'abdoment.

P. JAGUGisa

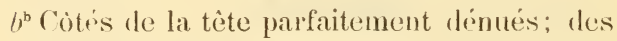
hordures fines blanches sur les plumes frontales.

Aburria$$
\text { P. BOLIVIIX.I }
$$

b" Des bordures blanches sur toutes les plumes de la tète.

\section{P. SELATERI}

\section{GENRE CIIM MIPETES}

" V'eau nue des coités de la tìte aussi large et aussi longue que dans les pénélopes vraies, le roux du dessous commençant sur la région jugrulaire.

Ch. T'sghuml

b Peau nue des côtés de la tîte étendue en dessous jusquau niveau du milieu de l'oeil, puis entourant finement l'oeil; le roux du dessous ne eommencant qu'au bas de la poitrine. 


\section{FAMILLE THINOCORIDA}

A Queue cunéiforme, à rectrices terminées dle blanc. Thrvocorus

B Queue arrondie, à rectrices non terminées dle blanc. ATTAgIs

\section{Gexre ThINOCORIS}

$a$ Chez le màle, cou cendré plombé séparé du blanc de la poitrine par une bande noire, une bordure noire sur les côtés et au-dessous du blanc de la gorge.

Th. orbignyanus

$b$ Chez le màle, devant du cou cendré pâle séparé en dessous du blanc de la poitrine par une bande noire réunie avec la bordure gulaire par une bande noire jassant le long du milieu du cou.

TH. Rumcivorus

\section{FIIILLE TETRAONIDE}

Bec court, épais, comprimé, à dos fort arqué; doigts longs, à ongles longs aigus et peu courbés; queue courte et arrondie; lores et tour des yeux plus ou moins dénués.

Odontuphorus

\section{GENhE ODONTOPIIOR US}

a Gorge et côtés de la tête noirs; dessous du corps roux.

O. speciosus

$b$ Gorge non noire.

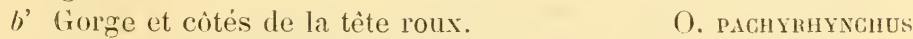

b" Gorge et côtés de la tète cendrés. O. strllatus

b" "Une raie noire transoculaire bordée des deux cotés d'une raie roux intense parcourant audessus et au-dessous des yeux.

O. Baldiviani 


\section{FaMILLE TINAMIDA}

A Pouce distinct.

A' Bee presque droit ou à peine courbé. à extrémité de la mâchoire fort courbée en le faisant très obtus; ongles courts et larges.

A. Queue courte, à rectrices larges, courertes par les tectrices jusqu'à leur extrémité ou les dépassant un peu.

Tinanus

$A B$ Queue nulle ou courte, les tectrices dépassant toujours l'extrémité des rectrices lorsqu'elles existent.

Cryptures

A" Bee courbé et atténué graduellement jusqu'à son extrémité qui est subaigü̈ ou arrondie au bout; queue nulle; ongles assez longs et médiocrement courbés.

B Pouce nul.

\section{Nothoprocta \\ Tinamotis}

\section{GeNRE WINAUUS}

a Sommet de la tête roux, fond du plumage olive. T. Ruricks

$b$ Sommet de la tite noiràtre, fond du plumage cendré.

T. KLeEI

c Sommet de la tête cendré strié de noir, dos brun olive, à région interscapulaire rougeâtre. 'T. Gurritus

d Sommet de la tête noir, une grosse tache brun roussitre derrière l'ouil.

T. ATROG.APILLES

\section{GeNRE CRYP'TUIRUS}

a Rémiges secondaires inmaculées.

a' Dessus du corps brun rougeatre; sommet de la tête fuligineux foncé, pâle sur la gorge; dessous roux, plumes des côtés du basventre bordées de fauve.
Dessus brun foncé un peu rougeatre; tète C. UBSOLETES

a" Dessus brun foncé un peu rougcatre; têto fuligineuse, à gorge blanche; plunes du basventre bordées de blanc.

a" Dessus roux brunâtre; tête plombèe, à gorge blanche; dessous gris, à plumes du basrentre bordées de blanc.

a"' Dessus brun roussâtre; tête ardoisé foncé lavé de rougeâtre au sommet, gorge blanche; 
poitrine cendré grisâtre, abrlomen blanchâtre,

le tout varié d'une vermiculation brune. C. Balston

b Rémiges secondaires variées d'une série de taches claires.

b' Dessus du corps non rayé en travers.

ba Brun fuligineux en entier, taches aux rémiges secondaires peu prononcées.

C. CINFHEUS

b6) Brun rougeâtre pâle tirant au violet, varié de quelques petites bordures noirâtres au milieu du dos et les scapulaires postérieures.

C. hubripes

b" Dessus du corps plus ou moins rayé en traver's.

ba Dos brun ocreux rayé en travers de noir; tectrices alaires plus noires, à raies ocreuses moins nombreuses.

C. Bakthetti

bb Bas du dos ct croupion rayés en travers de noir et de roux.

C. NOCTIYAges

\section{GeNhe MOTHOPROC'T}

a Région jugulaire et poitrine cendré immaculé. N. Branigki

b Rémiges secondaires roux cannelle rayé de noir. N. Gunvirostris

c Taille forte, poitrine maculée.

$c^{*}$ Ocelles jugulaires fauves entourées de noir sur un fond cendré.

N. TAGZANUWSKLI

c" Taches jugulaires brun noirâtres subcordiformes bordées de fauve pâle et de roussâtre sur un fond gris roussâtre sale.

N. Gommani

\section{FaMILLE PALAMEDEIDAE}

Aile armée de deux fortes épines cornées, front surmonté d'une longue baguette cornée. 


\section{FAMILLE RALLID瓜}

A Doigt médian arec l'ongle plus court que le tarse. Arsmoes

B Doigt mérlian arec l'ongle plus long que le tarse.

b' Arète dorsale de la mâchoire non élargie en plaque cutanće frontale.

$B A$ Bec plus long que la tète.

Rialus

$B B$ Bec plus court que la tête.

Porzaxi

B" Arète dorsale de la mâchoire élargie en une plaque frontale cutanée couvrant le front au moins jusquau milieu des yeux.

b. Doigts pourvus sur les còtes d'un rebord étroit et continu.

Ba Plaque frontale atténuée et acuminée en arrière.

Bb Plaque frontale large, arrondie ou coupée carrément en arrière.

Gallinela

bli Doigts garnis sur les còtés d'une membrane large partagée en festons.

Fulicis

\section{GENRE IRAMIDES}

$a$ P'oitrine et abdomen roux.

b Poitrine et abdomen plombé bleuatre.
A. GasenNexis
A. s.rR.ugure

\section{Gente RaLUS}

a Dessus du corps varié de grosies flammèches foncées.

$a^{\prime}$ Dessous du corps plombé.

$a^{\text {a }}$ Tectrices alaires olives.

R. PERUYIANES

$a^{\text {b }}$ Tectrices alaires rousses.

R. SEMIPLIMBEUS

a" Dessous du corps roussâtre.

$a^{\text {a }}$ Tectrices alaires grises.

R. CYPERETI

$a^{\mathrm{b}}$ Tectrices alaires rousses.

R. virgimanus

$b$ Dessus du corps immaculé.

b' Une tache rouge sur les còtés du bec.

li. C.Esil's

b" Point de rouge à la base du bec.

R. NIGRICANS

\section{Gente Porzhana}

a Dos et ailes tachetés de blane.

a) Taches blanches fines longitudinales; le de- 
vant du visage, une raic gulaire et une autre céphalique noirs.

P. CAROLINA

a" Taches blanches transversales; point de noir au visage et à la gorge.

P' BaWAGEXTS

$b$ Dos et ailes non tachetés de blanc.

b' Du roux sur la tète.

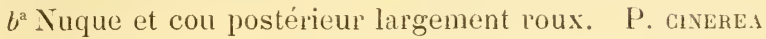

$b^{\text {h }}$ Le sommet de la tète et tout le dessous du corps roux.

P. Chyexyexsis

$b^{c}$ Tète, cou entier et poitrine roux rougeâtre. P. Hacxweldi

$b^{2}$ Une large strie rousse de chaque côté du front.

P. Faciatis

b" Point de roux sur la tête ni ailleur's, dessous du corps plombé.

P. erythrops

\section{Gente FuLici}

a Taille forte, scutelle frontale fort renflée jaune, bec rouge.

F. GIGANTEA

b Taille médiocre, scutelle frontale renflée jaune pàle, bec jaune rougeâtre.

F. ARDESIACA

\section{Finille PARRIDE}

Pli de l'aile armé d'une épine aiguë cornée, doigts très long's et grêles, à ongles très longs et fins.

Parra

\section{Fayille CEDICNEMIDE}

Pattes élevécs, à trois doigts; tête grosse, à oil gros de couleur claire; bec à arcte dorsale proéminente arrondic, courert à la base d'une cire jusqu'aux narines.

OLdicnemus 


\section{Famille CHARADRIID}

A Dos et ailes à couleurs métalliques très fortes. Tanelus

B Rien de métallique au plumage.

B' Pattes à pouce plus ou moins déreloppé.

$B A$ Bec graduellement atténué jusqu'au bout, sans enflure dorsale devant la fosse nasale, comprimé, légèrement recourbé en haut. STrepsilıs

$B B$ Bec droit, renflé devant la fosse nasale, à extrémité un peu courbée en bas.

Ba Pouce assez long, pointe du becémoussée. Aphriza

Bb Pouce court, fin, à ongle très petit, extrémité du bec pointue.

Soutatirola

B" Pouce nul.

BA Une épine cornée courte au pli de l'aile. Hoplopterus BB Épine alaire nulle.

Ba Bec très fin, à enflure teiminale très faible, doigts courts plus de deux fois moins longs que le tarse.

Oreophilus

Bb Dos du bec distinctement élevé au-devant de la fosse nasale.

$l^{1}$ Le dos et les tectrices alaires maculés. Charadrius $b^{2}$ Le dos et les tectrices alaites non maculés.

Egialitis

Bi Bec long, fort comprimé graduellement jusqu'à l'extrémité, à deux mandibules terminées en coin tranchant.

HenATOPUS

\section{GENRE VINELLUS}

a Pouce développé, une huppe cervicale.

V. OCCIDENTALIS

$b$ Pouce nul, point de huppe.

V. RESPLENDENS

\section{GenRE RGLLITIS}

a Deux bandes noires, dont une collaire et l'autre pectorale.

$b$ Une bande noire collaire.

6 ' Bec jaune à la base.

E. VOCIFERA

$b^{\mathrm{a}}$ Bec tout noir.

ha Bec épais, point de loux sur la tète et le cou.

E. Wilsonia

b३ Bec faible, du roux au cervix et sur les côtés du cou.

E. SEMIPALARTS

E. COLlaris

c Point de bande collaire.

E. NTrosi 


\section{Genre II EM IT(J)IS}

a Tout le plumage foncé presque uniforme.

II. ATER

$b$ Miroir alaire, poitrine et abdomen blanes, dos brun.

H. palliatus

\section{Famille SCOLOPACID}

A Pouce nul.

1' Pattes courtes.

$A A$ Doigt médian avec l'ongle plus court que

le tarse.

Gatidris

$A B$ Doigt médian avec l'ongle plus long que le tarse, des bandes blanchâtres en travers du sommet de la tête.

Phegornis

A" Pattes fort longues.

Hinantopus

B Pouce plus ou moins développé.

B' Doigts antérieur's réunis à la base par une membrane plus ou moins développée sans lobes dans leur partie libre.

BA Bec non élargi près de l'extrémité, à mâchoire courbée subitement au bout même.

$B a$ Bec épais, membranes interdigitales fort développées jusqu'au niveau de la première articulation.

Symphemla

$B b$ Bec faible, membrane rudimentaire entre le doigt médian et l'interne.

Ba Queue à rectrices presque égales, bec beaucoup plus long que la téte. Totanes

$B \beta$ Queue à rectrices plus ou moins étagées.

B1 Doigt médian avec l'ongle presque égal au tarse.

$B 2$ Doigt médiarı avec l'ongle moins long que le tarse.

$P^{\star} \quad$ Membrane entre le doigt extorne et le médian bien développée.

$P^{\star \star}$ Membrane entre le doigt externe et le médian presque nulle.

Actitis.

Tringites

BB Bec plus ou moins renflé dans sa partie terminale. 
Ba Bec droit ou peu courbé, à extrémité lisse.

Pa Pattes courtes.

$l^{*}$ Membranes interdigitales rulimentaires.

Tringit

$b^{\star \star}$ Membranes interdigitales plus développées.

Ereunetes

$B \beta$ Pattes élevées, membranes interdigitales, surtout l'externe, bien développées.

Bb Bec au moins deux fois aussi long que la tète, légèrement courbé en haut dans sa partic terminale.

LINUSA

Bc Bec beaucoup plus long que la tète, plus ou moins arqué.

Numenies

Bd Bec couvert d'une membrane molle, se plissant dans la partie terminale après la mort.

Ba Bec plus ou moins courbé à l'extrémité.

B $\beta$ Bec droit.

BI Doigt médian avec l'ongle plus court que le tarse.

Macrorhamphus

B:- Doigt médian avec l'ongle plus long que le tarse; une ligne fauve le long du milieu du sommet de la tète.

Gillinago

$B C$ Bec fort aminci à l'extrémité, fort recourbé en haut, membranes interdigitales fort déreloppées, dépassant beaucoup dans leur milieu la première phalange.

I." Membranes interdigitales fort développées, le reste des doigts bordé de lobes cutanés.

Phalaropes

\section{Gente: TO'THNUS}

a Fond du manteau gris, tacheté de blanchàtre et de noir.

$a^{\prime}$ Taille forte, pattes olives.

T. MELAYOLEUCOS

$a^{\prime \prime}$ Taille médiocre, pattes jaunitres.

T. FLAYIPES

b Fond du manteau noir olivaitre tachetéde blanc. 'T. somiranies

\section{GENRE TIRINGA}

a Dessous roux en noces, blanc en hiver; taille forte.

T. canutes 
$b$ Abdomen constamment blanc.

$b$ 'Sus-caudiales médianes largement noires.

ba Rectrices médianes considérablement plus longues que los autres of atténuérs il l'extrimité.

bu Taille médiocre.

T. ILACULATA

b $\beta$ Taille petite.

T. MINUTILLA

bb Réctrices médianes dépassant peu les autres.

T. Bairder

b)" Sus-caudales blanches.

T. FUSGICOLLIS

\section{Gexre Galding(i)}

a Ventre blane pur; taille petite.

b Ventre rayé de foncé; taille forte.

(i. ANDIN.

(i. JaMESON

\section{FAMILLE PSOPHIIDÆ}

Bec plus court que la tête, à narines ovalaires percées en travers; pattes élerées, à loigts courts; ongles courts et fort courbés; quene courte; tête et cou courcrts de plumules veloutées et érigées.

Psophls

\section{GENRE PSOPIIIA}

a Plumes longues du dos inférieur gris cendré. P. corfpitans

$b$ Plumes longues du dos inférieur blanches. P'. l.evcoptera

\section{FAMILLE ARAMID瓜}

Bec deux fois aussi long que la tête, comprimé, droit, à extrémité de la màchoire légèrement courbée; pattes élevées, à tarse plus long que le doigt médian; narines étroites percées en travers.

\section{FAMILE EURYPYGIDE}

Bec droit, comprimé, fendu jusqu'au-dessous des yeux, cou longr et mince; pattes peu élevées; plumage nuancé par bandes et par lignes, imitant les beaux papillons de nuit. 


\section{Fanilife ARDEID $\bar{A}$}

A Bec comprimé, aigu au bout; tibias dénués jusqu'à la moitié de la hauteur; còté postérieur du cou emplumé.

A' Cou long ct fin; pattes élevées, à doigt médian moins long que le tarse.

Ardea

A" Cou gros ; pattes médiocrement élevées, à doigt médian avec l'ongle plus long que le tarse.

Butorides

B Cou gros, doigt médian moins long que le tarse, à doigt médian avec l'ongle plus long que le tarse. Nycticorax

C Partie dénuée des tibias très courte; cou couvert de plumes longues, courrant le côté postérieur qui n'est que parsemé de duvet.

6" Tarse plus long que le doigt médian arec l'ongle; doigt interne plus long que l'externe; ongles fort courbés.

Tigrisoma

("' Tarse moins long que le doigrt médian avec l'ongle: ongles faibles et peu courbés.

ArDetTa

\section{Gevie IRIDEA}

a Plumage blanc.

$a$ ' Point de hupue, taille forte. A. LGRetat

a" Huppe cervicale abondante, taille petite. A. caxumissina

1) Plumage général ardoisé bleuâtre, à tête plus ou moins vincuse; jeune oiseau blanc.

A. C.ertlea

c Ardoisé bleuâtre en dessus, ì abdomen et poitrine blanes.

A. leevcogastra

$d$ Cendré en dessus, à sommet de la tète noir.

A. Cocol

$e$ Bec très long, huppe longue et abondante cendré bleuâtre, plumes collaires for't atténuées et frisées.

A. AGAIII

\section{GENRE TIGRISOVI}

a 'Tête et cou roux vif.

T. BRASILIENSE

$b$ Tète et cou non roux.

$b$ ' Gorge toute nue.

T. CABanisi

b" Gorge largement emplumée au milieu.

'T. SALHONI

\section{Gexre NyC'ICORAX}

a Tout le plumage blanchâtre, à sommet de la tête noir. 
$b$ Dos et ailes cendré bleuâtre, à grosses stries noires.

N. VIOLAGEUS

$c$ Sommet de la tête ot dos noir verdâtre métallique.

r. Plumes tongues de la hujpe blanches, a extrémité noire.

V. IVERIGANES

c" Plunes longues de la huppe blanches jusyu'an bout.

N. orscitris

\section{Finille CICONIIDE}

Bec énorme, élevé, tête et cou nus.

Mveteria

\section{FINIILE CANCROMIDE}

Bec très gros, large, terminé par un onglet, pattes médiocres, à pouce égal à la moitié des doigts antérieurs.

Caxcroma

\section{Famile Plataleide}

Bec long, trés plat et fort élargi près de l'extrémite.

\section{Familie TANTALIDE}

A Bec long, gros à la base, légèrement courbé ì l'extrémité, glabre; pattes très élevées.

B Bec long, plus ou moins courbé avec des sillons latéraux dans toute sa longueur.

B' 'Tibias dénués jusque près de la moitié de leur' longueur.

BA Tarse couvert de scutelles dans sa partic médiane.

$b^{\text {a }}$ Couleur des ailes et de la queue métallique.

$l^{\text {b }}$ Unicolores sans rien de métallique.

Eudogimus 
BB Tarse réticulé dans toute sa longueur, moins long que le doigt médian avec l'ongle.

Harpiprion

l' Tibias tris peu dénués au-dessus du talon, pattes of doigts comts, quene longur cumiformo.

Theristicts

\section{GENRE IIRPIPRION}

a Нирреं.

H. G.ERLLESCENS

b Non huppé.

II. GAYENYENSIS

\section{FAMILLE PHCENICOPTERIDAE}

Pattes très ćlevées, à doigts antérieurs palmés en contier, bords du bee garnis de lamelles cornees. Phomicopterus

" Lamelles de la mâchoire verticales. PH. igNipallatés

le Lamelles do la mâchoire horizontales, michoire fort attenues.

PH. axmixes

\section{FAUILLE FREGATIDAE}

Membranes interdigitales courtes, ailes très longues, queuc longue profondément fourchue. Frigata

\section{FinILLE PELECANIDÆ}

Bec très long et plat, terminé d'un onglet fort, sac gulaire tròs dilaté, non plumé.

\section{Finilie PHALACROCORACID $\&$}

Bec droit, comprimé, terminé par un crochet fort courbé, rectrices longues plates.

Phatagrocorax

a Quene à douze rectrices.

$a$ ' Toir en entier avec une bordure blanche autour de la partie dénuée de la gorge et des joues.

P11. brasillexsis 
a" Gorge, milieu de la poitrinc et abdomen blancs.

Pir. Bodganviliti

1) Queue à quatorze rectrices; gris, grosse tache blanche de charpue coté du cou.

Ph. Gamingi

\section{FamiLe SULIDAE}

Bec subconique, comprimé sur les còtés, à dos de la mâchoire arqué à l'extrémité, terminé en pointe.

\section{FAMILLE PLOTIDE}

Bec droit comprimé, aimu ì l'extrémité; queue longue et plate, à surface des rectrices ondulée. PLotus

\section{Fawile PHaEThONIDE}

Bec compriné, aigu au bout, deux rectrices médianes lort prolongées et tres etroites.

PH.ETHox

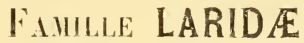

A Bec fort comprime en lame tranchante, à mandibule terminée en coin tranchant, dépassant beatcoup la mâchoire.

Rinvehous

B Partie postnasale du bec plus longue que la basale, à extrémité terminée en pointe; queue plus ou moins fourchue.

B.A Membranes interdigitales échancrées au milieu. STEкx.

BB Membranes interdigitales non échancrées. N.Exu

C Partie postnasale du bec plus courte que la basale, à dos arqué, l'extrémité plus ou moins courbée. $C A$ Queue coupée carrément. ('B) () puene fourchue.

D I'artie postnasale du bec fort raceourcie, à dos fort arqué, une cire basale jusqu'à l'extrémité des narines; queue à deux rectrices médianes plus longues que les autres. 


\section{GeNhe STERIA}

a Bec fin.

a' Rectrices externes fort prolongées et atténuées à l'extrémité.

$a^{a}$ D'un cendré perlé en dessous.

$a_{\alpha}$. Sommet de la tète noir.

-. macreri

$a \beta$ sommet de la tête noir, à front et sourcils blancs.

S. EXIL1S

$a^{\mathrm{b}}$ D'un blanc pur en dessous.

S. HIRLNUINACEA

a" Rectrices externes dépassant peu les subexternes.

$a^{\text {a }}$ Sommet de la tête blanc sans huppe noire, ligne transoculaire noire.

$a^{\mathrm{b}}$ Bec noir, front et sourcils blancs.

S. Trcieaui

S. SUPERGILIARIS

$b$ Bec élevé, plus ou moins épais.

b' Cendré perlé clair en dessus.

$b^{2}$ Bec jaune.

S. MAXINI

$b^{\mathrm{b}}$ Bec rouge.

S. ELEGANS

$b$ " Dos et bras fuligineux.

S. MAGNIROSTRIS

\section{Genke LARUS}

a Tout le plumage fuligineux presque uniforme.

L. MODESTS

$\checkmark$ 'Tète blanche en été, dos et ailes noirâtres.

L. Dominiciats

c Un capuchon foncé sur la tête en été.

c' Capuchon fuligineux, dos et ailes noirâtres, une bande noire antéapicale en travers du bec.

L. Belcher1

c" Capuchon noir.

I. SERRANUS

$c$ "' Capuchon brun, rémiges primaires blanches jusqu'au bout.

L. GLAUCODES

(.") Capuchon plombé fuligineux plus foncé que le dos.

L. ATRICILLAI

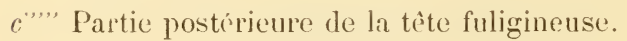

L. Firinklini

c.","' Capuchon cendré perlé plus clair que le dos.

L. CIRRHOCEPHALUS

\section{GeNre IEMA}

a Capuchon céphalique plombé bordé en dessous de noir.

X. SABIXII

b Capuchon céphalique plombé, à bordure frontale blancbe.

X. FURGATUM

\section{GENRE STERCORARIUS}

( Miroir alaire blanc.

S. CHILENSIS

1) Point tle miroir alaire.

S. PONATORHINUS 


\section{FaMILLE PROCELLARIIDA}

A Narines séparées entre elles, pouce nul.

DJOMEDEA

B Les deux narines dans un tube commun.

BA Pouce plus ou moins distinct.

i' Orifices nasaux situés au fond du tube.

$D^{\mathrm{a}}$ Bec large déprimé dans la partie basale. DAptriox

$P^{\mathrm{b}}$ Bec médiocre, plus long et plus faible. Thamassona

$B^{\circ}$ Bec court comprimé, ongles petits comprimés.

$B_{\alpha}$ Queue faiblement échancrée.

Oceanites

$B \beta$ Queue profondément fourchue.

Cryochorea

b" Orifices nasaux ouverts à l'extrémité du tube, séparés entre eux.

Nectris

BB Pouce nul, narines basales.

HaLONROMA

\section{Gente DIOHEDEA}

a Queve noire, à base des rectrices blanche.

D. IRROR.ITA

b Queue blanche, à rectrices terminées de brun foncé sur leur barbe externe et sur les deux barbes dans les médianes.

D. Exuhass

\section{FAMILLE ANATIDÆE}

A Tarse plus long que le doigt médian arec l'ongle. A' Dentelure de la màchoire non visible à l'extérieur.

$A A$ Bec élevé à la base, deux fois moins long que la tête.

Bervich.

$A B$ Bec moins de deux fois plus court que la tête.

Chevalopex

B Tarse moins long que le doigt médian arec l'ongle.

$b^{\prime}$ Les lores et le tour des yeux non emplumés, comverts de caroncules charmues.

C.jirix.

$b$." Toute la tête empluméc.

BA Pli de l'aile armé d'une épine cornée. Merianetra

$B B$ Aile sans armure.

Ba Membrane du ponce étroite.

$B \propto$ Bec graduellement élargi vers l'extrémité. 
li $\beta$ lBec non élargi vers l'extrémité.

B1 Tarse élevé, doigts longs, point

de miroir métallique.

I)ENDHOCigni

$B$ ? Doigts courts, miroir alaire métallique.

Bx Queue assez longue, à rectrices médianes plus ou moins atténuées.

I) AFILA

B.xxQueue à rectrices peu iné-r Aras gales.

Querquedula

Bb Membrane du pouce élargie.

Ba. Queue courte à rectrices normales. Fuligula

$B \beta$ Queue à rectrices atténuées et raides. Erisinatura

\section{Gexte DeNDROCYGNa}

a 'Tout l'abdomen noir, poitrine grise ondulée de fauve.

D. DISCOLOR

b Milieu de l'abdomen noir, devant de la tête blanchàtre.

I). VIDUATA

c Abdomen roux.

D. FULTA

\section{GENRE Q QUERQYUDULA}

a Tectrices alaires bleues, miroir rert.

Q. (TINOPTERA

b) Tectrices alaires grises.

b' '.ommet de la tìte noir, còtés et gorge blanes. Q. puxs

b)" Toute la tîte ondulée finement de noir et de

fauve.

Q. oxiptik.

\section{GeNre DIFHL}

a Cutés de la tête et gorge blancs, queue blanchatre.

D. B.LH.HIEXSIS

b Cotés de la tète fauves striés finement de brun; rectrices brunes bordées de fauve.

D. OxILRA

\section{GEYAE MERGIMETT}

a Mâle, à poitrine et abdomen blanchâtres striés de noir.

M. LEWCOGEYIS

b Vâle, à région jugulaire et flancs noirs, poitrine et ablomen gris vermicules de foncé.

M. TURNeli 


\section{FIMILLE HELIORNITHID F}

Doigts garnis de membranes festonnees; queue

longue et plate, fort arrondie à l'extrémité. Hewonxs

\section{FaWILLE PODICEPITIDA}

A Ailes normales.

A' Bec plus ou moins faible.

A.1 Tète huppée.

Aa Taille forte.

Ab Taille petite.

Echmophore

Poniceps

Al) T'ète non huppée, taille petite.

TACHYBAPTL

PODILTMBL:

.' Bec éleré, à dos fort arqué.

Cextropelin

$\mathbf{B}$ Ailes petites.

\section{GENRE PODICEPS}

a Dussous du corps roux, tete noire, avec une grosse tache auriculaire blanche variee de noir. I'. liobs.dxm

b Tout le dessous blanc, dessus de la tite gris, à nuque jlus foncée, des plumules straminces sur' la région auriculaire.

\section{FAMILLE APTENODYTIDE}

Ailes impropres an vol couvertes de plumules squamiformes; bec élevé comprimé, à dos de la màchoire fort arqué à l'extrémité.

\section{FAMLLE RHEID㞋}

Pattes éleries, à trois rloigts; ailes impropres au vol. 
Dans le texte, la Palamédée est omise. Conme l'oiscau est généralement connu, il nous suffit d'indiquer la notice suivante :

\section{0. - Palamedea cornuta}

L., S. N., I, P. 232 - Scl. et Salv., P. Z. S., 1866, p. 200; 1873 , p. 304 .

Ucayali supérieur, Huallaga (BAR'TLETT).

Dans le deuxieme volume, p. 296, 29e et 30 e lignes, au lieu de 55 millimètres, lise 15 millimètres. 


\title{
TABLE ALPHABÉTIQUE ${ }^{(*)}$
}

\author{
DES ESPECES ET DES SYNONYMES
}

A

Aburria carunculala............... Tomes III

Acanthylis brachycerca............. I

Pages $27 \%$

brachyura ............. I

229

229

cinereicauda ............. I

229

collaris.............. I

231

rutila.............. I

Accipiter bicolor............... I

229

165

erythrocnemis........... I 163

haliä̈tus............. I 127

pileatus.............. I 166

sexfasciatus............. I 165

Acestrura micrura............... I 308

Mulsanti............ I 306

Acestura Mulsanti............... I

Acroleptes saturata.............. II

Actidromas brevirostris ........... III

Actinodromus Bairdi.............. III

Actitis bartramius .............. III

macularia................ III 369

macularius............... III $\quad 369$

Actiturus baitramius.............. III 371

navius .................... III 371

rufescens.............. III 371

Adelarus Belcheri............... III

Adelomyia chlorospila............. I

inornata.............. I $\quad 359$

maculata............. I 361

(*) Lers noms spécifiques adoptés dans le texte sont imprimés en italiques. 
Adelomyia melanogenys ............. Tomes I

Echmophorus major............... III

Pages 361

Egialitis collaris.................. III

492

nirosa................. III

341

semipalmala............. III

346

semipalmatus.............. III

34.5

rocifera .............. III

345

vociferus............... III

$34:$

IVilsonia................ III

342

34:3

Agamia picta.................. III

396

Agapornis colestis

II I

214

cyanopterus..............

21 ?

guianensis.

212

Agelaus thilius...................

III

424

Agelaius melanicterus...............

thilius ...................

xanthocarpus..............

Aglaractis Castelnaudi. ...............

Castelnaui................ .

caumatonota...............

caumatonotus.............

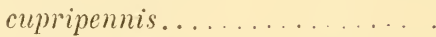

olivaceo-cauda........... . .

III

426

II

12.1

424

343

313

342

342

340

312

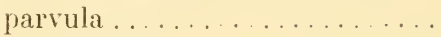

Aglaia caruleocephala ... . .........

340

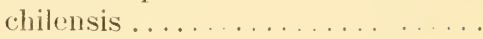

4\%

eyanicollis.

457

$47 \%$

cyanocephala

490

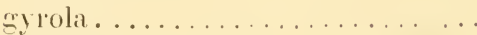

gyrolö̈des. . . . . . . . . . . . .

163

463

melanotis . ...............

459

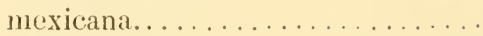

nigricincta . . . . . . . . . . .

nigriventris

161

peruviana.................

Schranki ... ............

striata...................

Yassori . . . . . . . . . . . .

Wilsoni.

yeni .................. .

Aglaiactis requatorialis.............

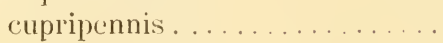

471

48 ?

463

459

188

454

471

4 茫

390

341

417

$1: 1$

personatus. ... . . . . . . .

Agriornis albicautu. . . . . . . . . . . .

insotens. .

18:

186

maritima. . . ...........

183

pollens.................

18. 
Agriornis solitaria ............... Tomes II

Agyrtria Bartlelti................ I

Pages 183

fiuriatilis.............. I

403

401

media.................

meliphila... .............

il'

111

phrobe................ I

414

Ajaja rosea.................. III

$41: 2$

Alauda bonariensis. . . . . . . . . . . . . . . I

160

cunicularia ............... II

93

erythropterus.............. II

93

fissirostris................ II

93

rufa................... I

460

tenuirostris................ II

Aleedo amazona................. III

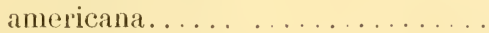

III

bicolor . .................

III

brasiliensis

III

Cabanisi .................. III

$10:$

cinerea ...................

III

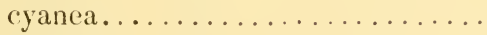

III

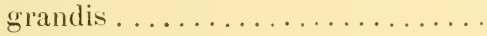

III

inda

III

$104,10 \%$

maculata.................

III

103

105

rubescens .................

III

stellata ...................

III

104

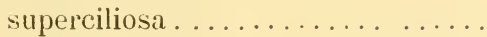

III

surinamensis............. III

100

100

19.2

103

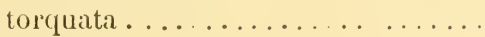

III

103

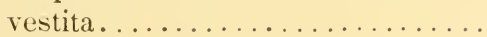

III

102

100

106

103

viridis $\ldots \ldots \ldots \ldots \ldots \ldots \ldots \ldots$.

III

100

viridirufa. . . . . . . . . . . .

III

$10 \%$

105

103

Amazilia amazicula . . . . . . . . . . .

latirostris ................

Lessoni..................

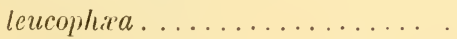

pristina...

Amazona icterocephala................

Amazonis bicolor....................

Amblycercus Prevosti...............

Amblyrhamphus nigerrimus...........

Ampelio arcuatus..................

Ampelion rufaxilla................

Ampelis arcuatus................. 
Ampelis elegans.

maynana.

Vierremi

Riefferi.

rubrocristatil

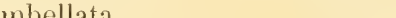

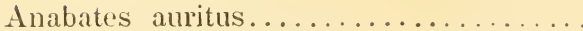

Boissonneaui ..............

erythrocercus..............

lineaticeps................

melanorhynchus ...........

montanus.................

ochralæmus ..............

pulvericolor ..............

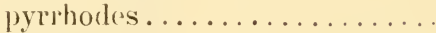

rufifrons.................

ruficaudatus . . ...........

ruficaudus.................

Sclateri................

squamiger...............

striaticollis..............

subulatus ...............................

Anabazenops Cabanisi................

rufosuperciliatus........... rufosuperciliatus Cabanisi.... ruficollis................

temporalis..............

Anaretes agilis....................

albocristatus. .........................

nigricristatus.............

parulus..................

Anas bahantensis.................

bicolor. ...................

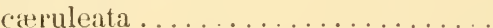

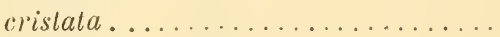

caudacuta..................

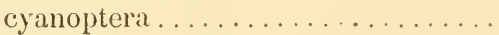

fimbriata .................

fulva.....................

ilathera .....................

leucogenys................

moschata.................

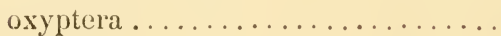

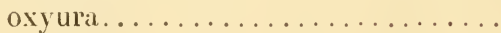

platalea .................

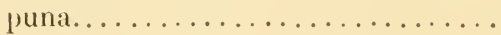

Tomes II

$\begin{array}{ll}\text { Tomes } & \text { II } \\ & \text { II }\end{array}$

Pages 378

385

374

375

381

383

381

391

145

145

$15 \%$

147

148

153

150

126

151

1.:3

1.5

15i)

151

164

153

149

$15 \pi, 158$

157

158

160

159

24:

$241,55 \%$

$55 \%$

239

482

471

475

173

481

474

483

170

483

486

$47:$

476

481

480

III

4 is 
Anas pyrrhogaster................ Tonies III

Rafllesi .................. III

Pages 473

rubrirostris. ............... [II

475

specularoïdes. . . . . . . . . . .

483

spinicauda............... II!

$17: 3$

urophasianus.............. . .

伿 1

viluata................. III

483

virgata................. III

471

470

Ancisthrops lineaticeps............. II

147

Andigena cucullatus.

157

hypoglaucus...............

III

157

laminirostris.

III

158

Androglossa amazonica.............

III

224

farinosa. . . . . . . . . . . .

III

$221)$

festiva ................

III

293

mercenaria............

III

221

ochrocephala ............

295

Anisognathus lacrymosus............

III

481

Anous inca.....................

II

446

Anser anticola.

III

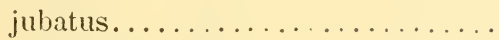

III

467

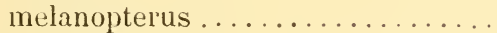

III

468

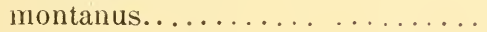

III

467

III

467

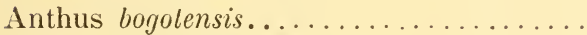

brevirostris.

I

457

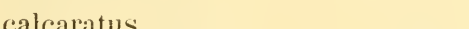

chii. ...................

correndera.................

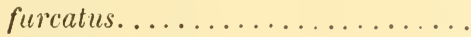

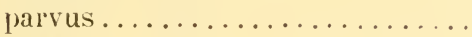

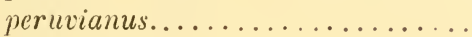

rufescens . . ...............

rufus ..................

Antrostomus requicaudatus............

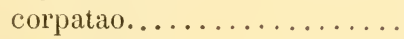

maculicaudus .............

nigrescens .............

ocellatus................

parvulus ................

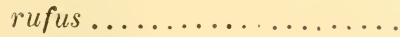

rutilus................

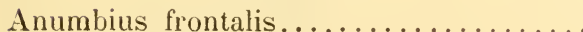

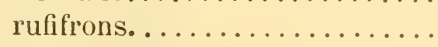

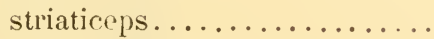

Aphantochroa gularis...............

hyposticta.............

Aphobus Chopi....................

Aphriza borealis................. 
Aphriza Townsendi

Tomes III

virgata.

Aquila americana.

Aquila balbusardus.

hpacenata. . . n

buzon ........................

niaculosa..................

megaloptera ..............

milvoüles.................

pezopora. ................

picta

piscatrix

tyrannus

urubitinga

urutaurana

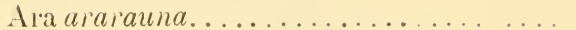

brasiliensis cyaneo-crocea.........

brasiliensis viridis.

Buffoni.......................

castaneifrons ..................

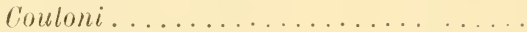

erythrochlorus. . . . . . . . . . . .

jamä̈censis................

jamaïcensis cyano-crocea .........

macao. ......................

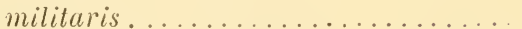

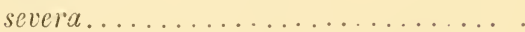

severus .....................

Aramides cayennensis...............

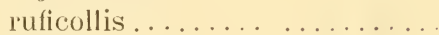

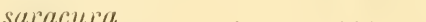

Aramus scolopaceus..................

Arara aracanga ................. .

ararauna..................

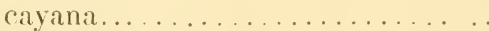

Aratinga guianensis. . . . . . . . . . . .

melanura.................

nobilis..................

xanthoptera. .............

Arenaria grisea..................

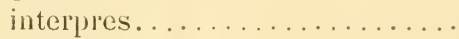

vulgaris. .........................

Ardea requinoctialis ...............

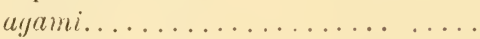

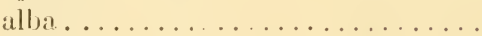

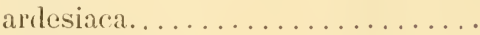

brasiliensis...............

brasiliensis candida...........
III

Pages 3 is

318

127

$12 \%$

115

110

169

101

111

97

104

$1: 7$

134

101

135

193

193

194

191

191

195

194

192

193

$19 \%$

191

194

191

318

315

319

387

19.2

193

199

199

203

199

207

$35: 3$

3 19

353

393

396

ils

39 '

401

391 
Ardea buccinator.

Tomes III

Cabanisi

III

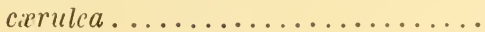

III

cirrulescens.

III

callocephala ...............

III

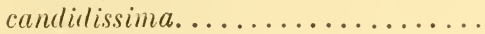

III

carolinensis ................

III

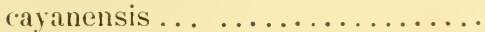

III

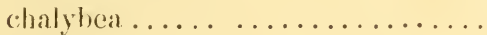

III

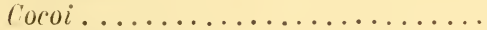

III

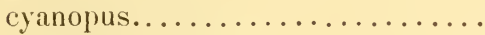

III

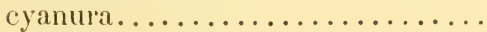

III

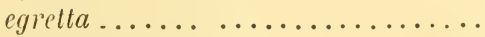

III

erythromelas ................

III

fasciata ..................

III

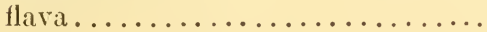

III

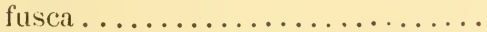

III

fuscicollis................

III

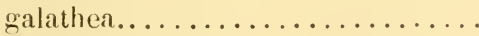

III

Gardeni.....................

helias.

III

involucris . ..................

III

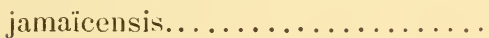

III

leuce ...................

III

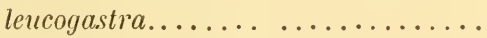

lineata..................

major.

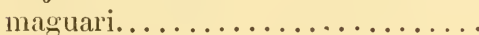

marmorata .................

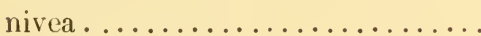

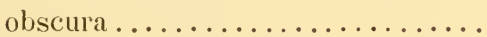

ohula .....................

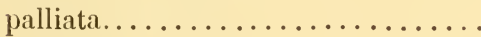

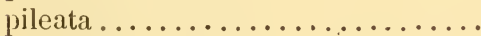

plumbea ................

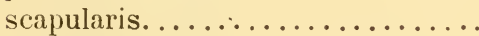

scolopacea ..................

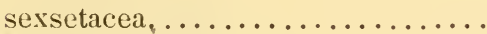

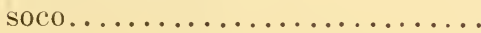

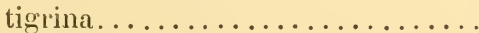

tula...................

violacea. .................

virescens................

Ardeola erythromelas..............

\section{III}

III

III

III

III

III

III

III

III

III

III

III

III

III

III

III

III

III

III

III

III

III

III

III

Pages 385

40 '

39 ', 397

390,394

405

393

393

405

39 '

390

39 í

397

391

399

401

401

396

398

391

407

388

399

$40 . ;$

391

395

401

390

390

401

393

406

393

390

408

390,394

398

387

405

$390, \quad 101$

401

393

105

397

399

399

399

399

involucris................ 
Arremon Abeillei................. Tomes II

affinis ................. II

Pages 534

assimilis............ II

530

erythrorhynchus............

531

frontalis.................

535

nigriceps...................

529

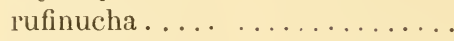

535

superciliaris

525

$51 \%$

Arundinicola leucocephala.............

207

Asio accipitrinus..................

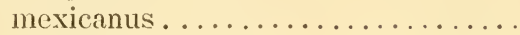

sandwichensis.................

193

192

193

Asthenurus rufiventris............. III

63

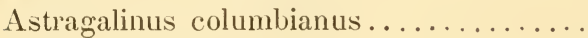

III

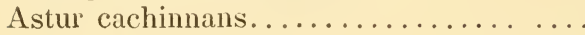

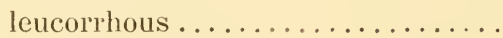

macrorhynchus.............

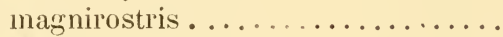

Mirandollei..................

poliogaster.................

Asturina Azare...................

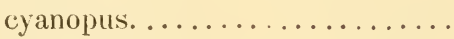

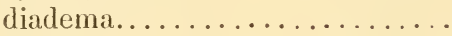

leucorrhoa..............

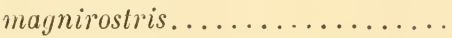

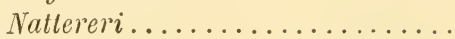

schistacea...............

superciliaris. .............

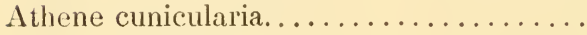

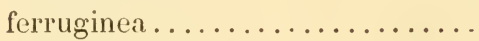

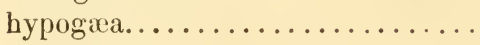

melanota................

minutissima.............

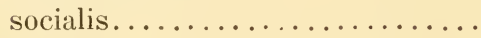

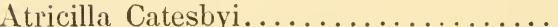

megalopterus .............

micropterus ............. III

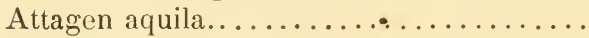

Attagis Gayji.......................

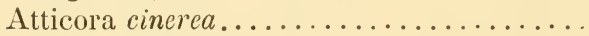

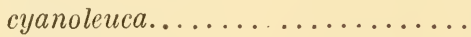

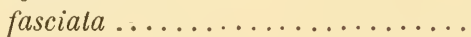

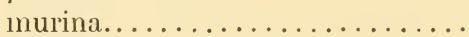

tibialis....................

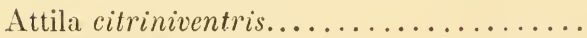

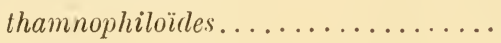

torridus....................

Aulacops hematopygus ............

III

284 
Aulacorhamphus atrogularis......... Tomes III

Pages 159

citruleocinctus........ III

derbianus............ III

160

hamalopyyius..........

158

Aulacorhynchus atrogularis. ...........

III

161

caruleocinctus .........

159

derbianus...........

160

158

369

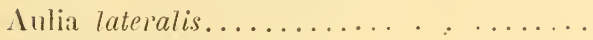

III

III

15\%

Automolus echinatus...............

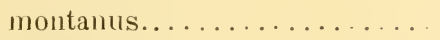

153

ochrolizmus ...............

150

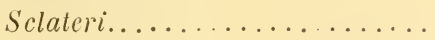

151

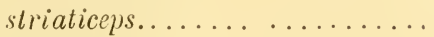

subulatus..............

Avocettinus carolus

Barbican de Cayenne.. . . . . . . . . . . .

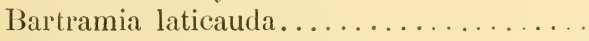

Baryphonus cyanocephalus............

cyanogaster..............

108

ruficapillus..............

III

112

semirufus...............

III

112

III

111

Baryphthengus ruficapillus............

III

Basileuterus biviltatus. ..............

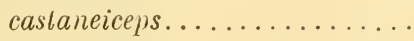

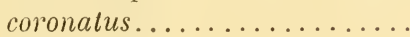

chrysogaster............

diachlorus. ..............

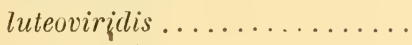

nigricapillus............

nigricristatus ...........

semicervinus ............

trifasciatus .............

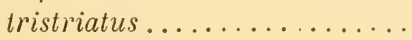

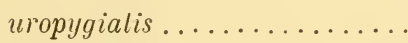

Bathmiderus albogriseus ...........

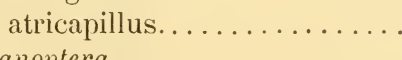

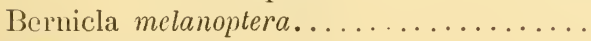

Bethylus medius. ................

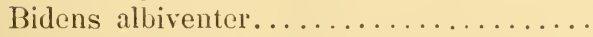

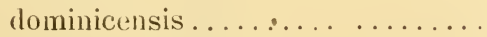


Biziura ferruginea.................. Tomes III

Blasipus Bridgesi................ III

Bolborhynchus andicola............ III

Piges 485

III

aurifrons............. III

211

orbignesius........... III

$: 10$

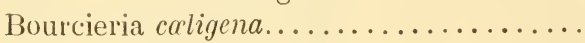

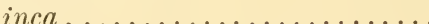

insectivora

Brachycex albogularis..............

Brachygalba albigularis ..............

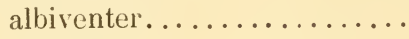

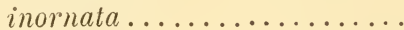

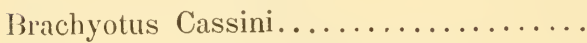
palustris americanus........

Brotogerys jugularis................

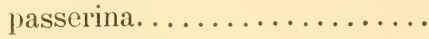

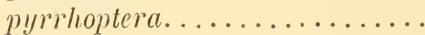

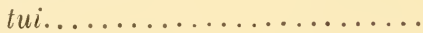

III

virescens................

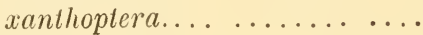

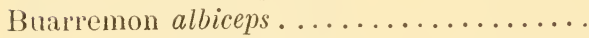

assimilis................

brunneinuchus .............

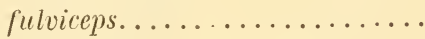

532

latinuchus...............

521

melanolæunus.............

mystacalis..............

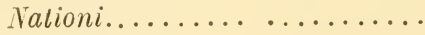

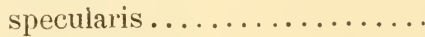

Taczanowskii.............

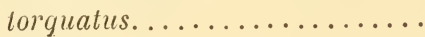

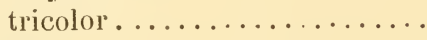

Bubo clamator.

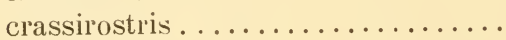

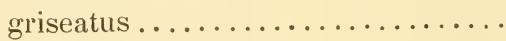

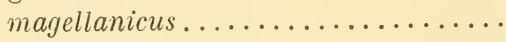

virginianus . ................

virginianus $[v$.$] magellanicus......$

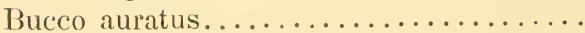

aurovirens................

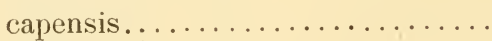

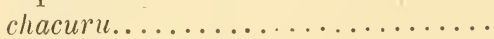

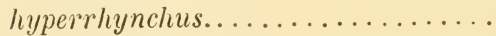

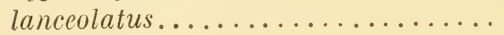

macrodactylus ................ 
Bucco nigrifrons................ Tomes III

peruvianus............... III

Pages 133

picatus

III

138

pulmentum.

124

rufus.

III

$1: 6$

strigillatus $\ldots \ldots \ldots$

III

130

strigillatus .............. II

129

Busarellus nigricollis .

111

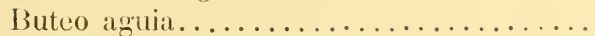

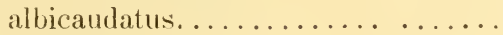

brachyurus. . . . . . . . . . .

busarellus.

134

115

118

erylhronotus.

111

115

Kaupi................. I

117

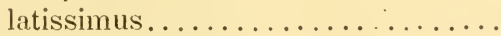

113

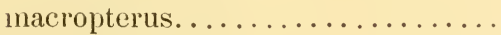

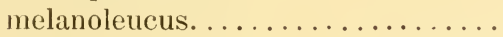

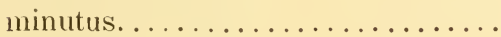

nigricollis.

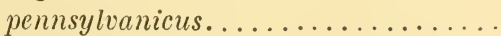

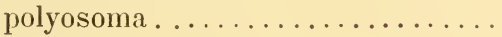

tricolor . . . . . . . . . . . . . . . .

varius.

169

118

118

111

113

115

115

115

111

111

118

483

397

Butorides cyanurus.................

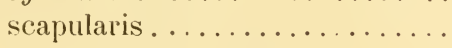

398

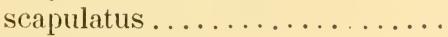

398

virescens .

Cabure.................... I

Cacicus Alfredi..................

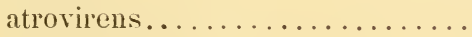

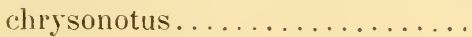

413

cristatus................

holosericeus ...............

persicus.................

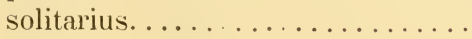

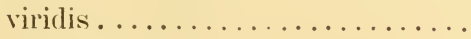

yuracares ................

Caïca Barrabandi.................. 
Caïca melanocephala ................ Tomes III

xanthoineros ................ III

Cairina Marianæ................ III

moschata................ III

sylvestris................ III

III

Pages 226

226

472

472

479

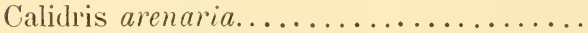

353

grisea minor.

353

tringoïdes.

III

353

Calipareus kalipareus..............

III

493

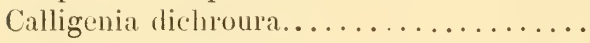

osculans ................

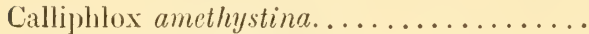

amethystoïdes . . . . . . . . . .

III

377

376

317

317

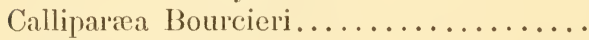

452

Callirhynchus peruvianus.............

512

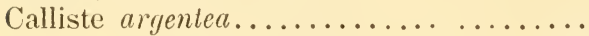

argentea viridicollis............

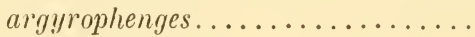

atricærulea .................

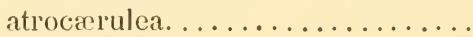

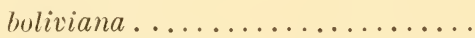

castaneoventris .............

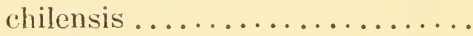

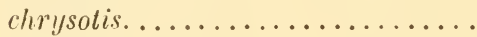

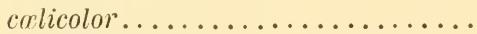

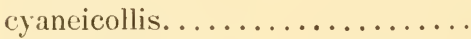

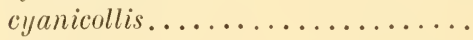

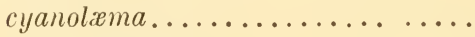

cyanoventris..............

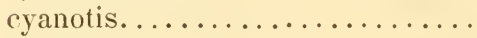

frugilegus ................

fulvicervix...............

gyroloïdes.................

Hannahix .................

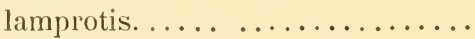

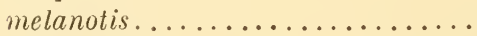

nigricincta..............

nigriviridis . . . . . . . . . . .

nigriviridis Berlepschi..........

Parzudatii.................

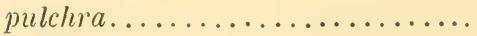

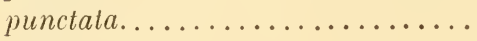

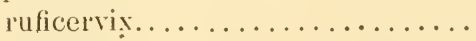

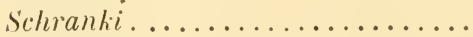

thalassina ................

Vassori....................

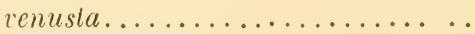

santhocephala..............

II

446

468

468

456

456

461

451

457

II

511

458

472

472

463

463

473

488

465

463

472

476

473

471

469

$40 ิ 9$

474

462

460

465

159

471

454

II

176

II

II

475,476 
Calliste xanthogastra. 'Tomes II

Pages 461

yeni.

Callospiza boliviana............. II

464

gyrola ... . . . . . . . . . II

463

pulchra............. II

462

punctata............. II

460

Schranki.

459

xanthocephala

475

yeni

457

312

Calothorax Fanny...................

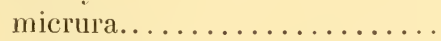

$30 \mathrm{~s}$

Mulsanti.................

Yarelli.................

Yarrelli.................

Calurus auriceps..................

pavoninus.

II

II

I

I

306

1

311

I

311

III

175

III

Camarhynchus cinere̊us..............

cinerea $\ldots \ldots \ldots \ldots \ldots \ldots \ldots$

III

175,176

III

Camarophagus leucoptera.............

Campephilus albirostris..............

lixmatogaster.............

melanoleucus..............

rubricollis ..............

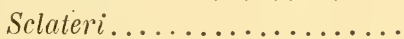

trachelopyrus.............

Campylorhynchus balteatus............

fasciatus............

liypostictus...........

cinnamomeus.

Campylopterus equatorialis............

largipennis............

obscurus...............

Cancroma cancrophaga..............

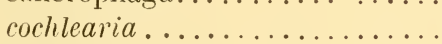

II

III

III

III

III

III

Cancrophaga bahamensis.............

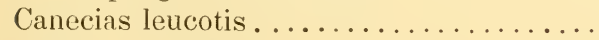

Capito amazonicus...................

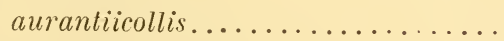

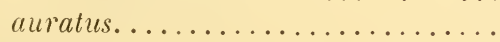

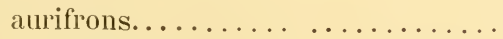

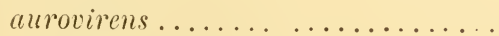

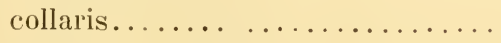

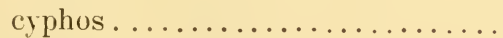

erythrocephalus..............

III

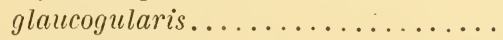

III

lanceolatus..................

III

leucotis...................

III 
Capito melanotis ... ............... Tomes III

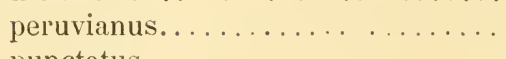

III

Pages 129, 1:1

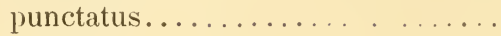

III

138

senilis

III

138

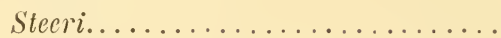

130

Tschudii.

III

143

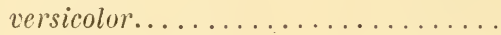

III

$14:$

III

111

Caprimulgus acutipennis............

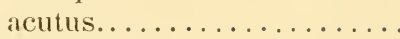

iequicaudatus ............

albicollis .............

americanus . . ...........

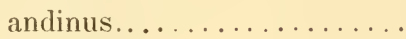

bifasciatus.............

brasilianus..............

brasiliensis............

campestris............

conterminus .............

cornutus.............. .

decussatus..............

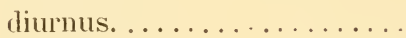

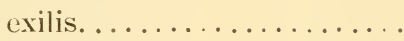

grallarius... ..........

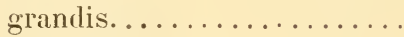

guianensis ..............

hirundinaceus. ..........

jamaïcensis ............

laticaudatus. ............

longicaudatus ............

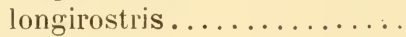

minor ..................

nacunda.............

nigrescens .............

ocellatus...............

parvulus...............

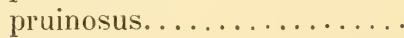

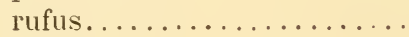

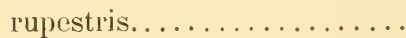

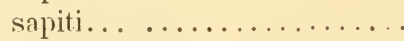

semitorquatus............

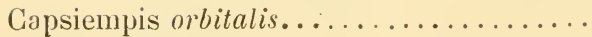

Carbo albigula..................

Bougainvillei...............

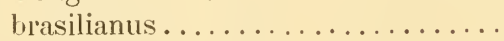

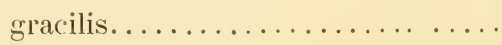

Cardinalis purpureus...............

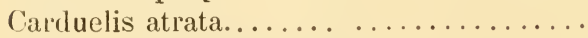

columbianus.

\section{I}

21:

$21:$

을

226

226

221

221

215

215

$? 08$

․ㄹ 1

207

221

208

212

226

20 '

$2: 6$

212

207

226

205

221

217

208

218

215

217

210

219

214

21?

212,218 
Carenochrous Dresseri................ Tomes II

Pages 5:8

latinuchus............ II

$5 \% 1$

melanolxmus............ II

525

Scebohmi.............. II

$5: 7$

Taczanoustii. .......... II

526

tricolor................. II

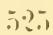

Carina moschata................ III

17:

Cariornis erythrurus . . . . . . . . . . II

301

phoenicurus ............. II

$30:$

Carnifex naso .................. I

160

Carpornis arcuata............... II

$: 379$

rubricristata............ II 38 I

Cassiculus leucorhamphus........... II

413

Prevosti .............. II

115

solitarius . ............. II

415

Cassicus affinis.................. II

41 't

Alfredi................... II

407

angustifrons............. II

410

ater................. II

435

atrovirens............. II

406

chrysonotus.............. II

412

crassirostris ............ . II

11 1

cristatus .............. II

404

Devillei................ . . II

403

flavicrissus.............. II

411

414

hæmorrhous............ II

415

holosericeus............... II

411

icteronotus .............. . . II

$40 ?$

latirostris............... II

leucorhamplus............ II

413

luteus............... . II

411

niger....................

435

nigerrimus. . ............

415

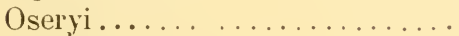

402

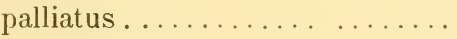

435

persicus...................

411

Prevosti................ II

ruber................ II

415

414

solitarius................. . . II

415

viridis ................ . II

405

vitellinus.............. II

411

yuracares ............. II

403

Yuracarium ............. If

mexicanus. ... ........... . II

oryzivora ............. II

Catamblyrhynchus diadema

Catamenia analis. 
Catamenia analoüles................. Tomes III

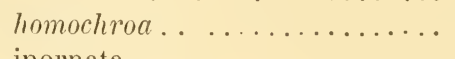

Pages 20

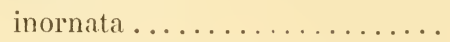

III

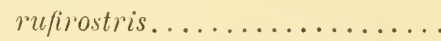

III

Catarracta parasita [v.] camtschatica. .....

Catharista aura..................

urubu.................

Catharistes atratus................

Catharies aura.................

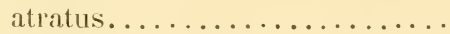

brasiliensis . . ............

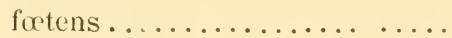

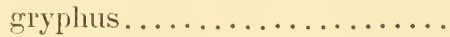

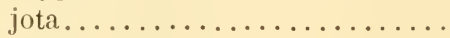

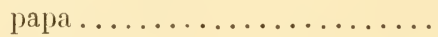

Ricordi................

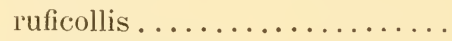

septentrionalis .............

urbicola.................

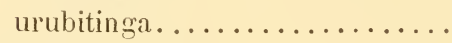

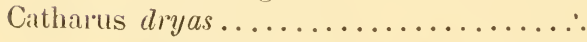

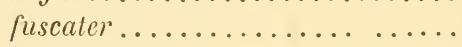

maculatus.................

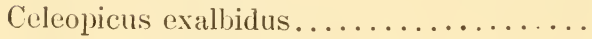

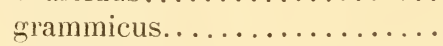

jumana.................

tinnunculus................

Celcus citrinus...................

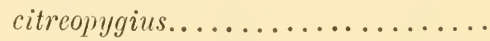

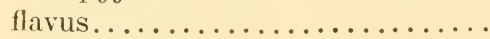

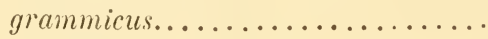

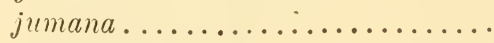

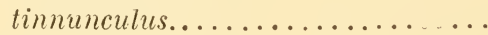

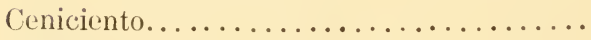

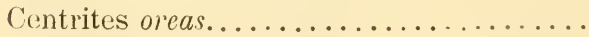

Centropelma microptera.............

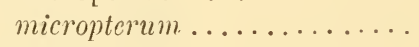

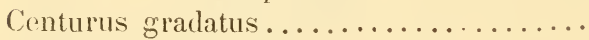

Cephalopterus ornalus...............

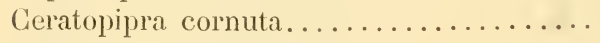

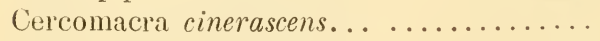

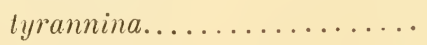

Cerchneis cinnamomina.............

Certhia americana atricapilla..........

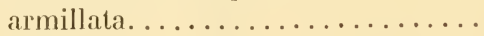

brasiliensis cerrulea...........

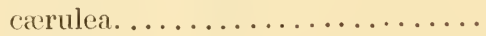

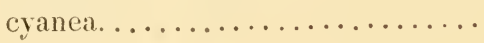

III

8', 89,91 II 551 
Certhia cyanogastra.............. Tomes I

Pages 436

flavipes............... I

436

ochrochlora............... I

spiza................. I

437

surinamensis............ I

435

437

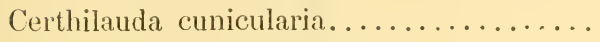

Frobeni............. II

95

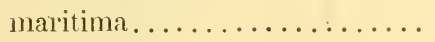

101

tenuirostris. ............

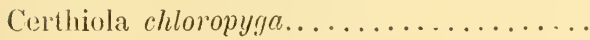

luteola................

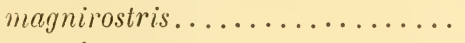

peruviana ...............

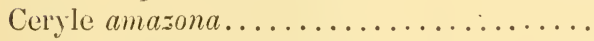

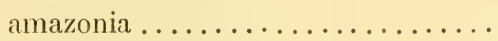

411

439

102

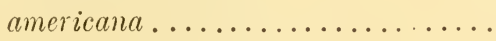

102

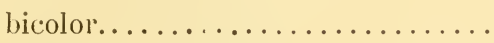

105

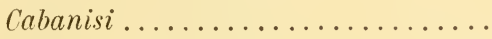

103

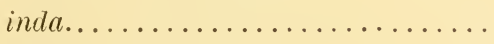

104

superciliosa.................

103

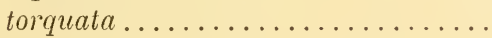

106

100

Chacaru.

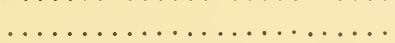

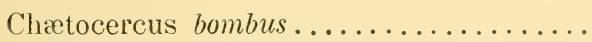

Chatura brunneitorques..............

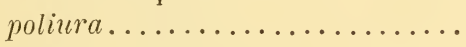

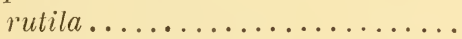

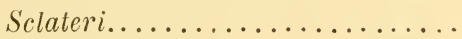

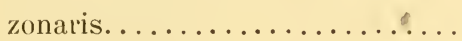

Chalcothraupis argentea............

atrocrerulea ...........

nigricincta............

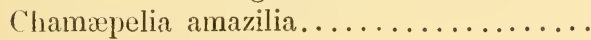

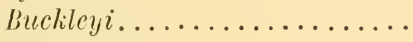

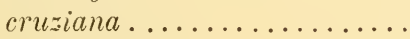

gracilis ..............

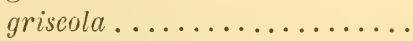

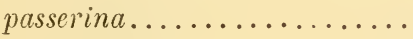

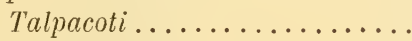

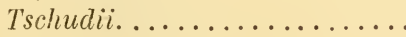

Chamæza nobilis.

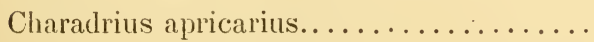


Charadrius cinclus ................ Tomes III

collaris............... III

Pages 349

crassirostris......... III

344,319

hiaticula.............. III

34.3

hypomelanus.

34;

hypoinelas. .

III

339

jamaïcensis . . .

III

339

342

larvatus . . . . . . . . . . . . .

34 '

næevius .............

338

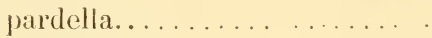

339

pluvialis...............

340

resplendens. . .............

336

rubidus.................

353

ruficollis. ...............

347

semipalmatus . . . . . . . . . . .

345

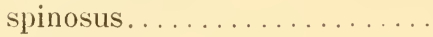

335

squatarola ...............

339

stolatus.

335

subtridactylus. .......... III

338

torquatus................

342

virginianus . . . . . . . . . .

III

virginicus..............

III

340

vociferus . . . . . . . . . . .

III

340

wilsonius ...............

III

31 ?

Winterfeldi . . . . . . . . . . . .

III

3 亿3

III

Chaunornis pulmentum............ III

348

126

Chelidoptera tenebrosa............. III

Chenalopex jubata............... III

137

468

Chiromacheris manacus................

349

Chiroxiphia regina.............. II

348

Chlö̈phaga melanoptera............ III

$46 \%$

Chloroceryle bicolor.............. III

Chlorochrysa calliparæa............ II

Chloranas albilinea................ III

103

$4 \div 2$

232

plumbea.................. III

2:1't

subvinacea............. III

936

vinacea.............. III

Chloronerpes callonotus............. III

235

callonotus peruvianus...... III

canipileus............. III

sil

s:

III

chlorocephalus... . .......

III

8'

tlavigula...............

III

sit

flavigularis.............

III

fumigatus .............

hæmatostigma..........

III

hilaris ..............

leucolxmus................

Chlorophanes atricapilla............ 
Chlorophonia Torrejoni............ Tomes II

Pages 438

viriclis .......... II

438

Chloropicus chlorocephalus........... III

81

Isıdori.............. . . III

85

leucolamus. ......... III

85

Chloropipo unicolor..................

335

Chlorospingus albitemporalis. ......... II

513

auricularis........... II

519

Berlepschic........... II

$5 ? 1$

castaneicollis........... II

520

chrysogaster........... II

515

cinereocephalus............

515

flavigularis ..............

514

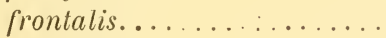

517

leucogaster..............

518

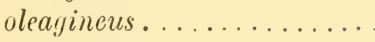

516

postocularis ..........

513

superciliaris............

517

xanthophthalmus..........

522

Chlorospiza Aldunati...............

erythronota .............

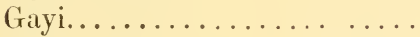

Chlorostilbon angustipennis..........

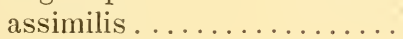

brevicaudatus.............

melanorhynchus...........

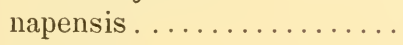

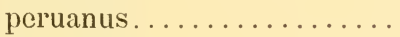

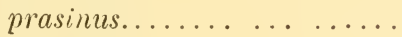

pumilus................

smaragdina.............

Chordeiles acutipennis.............

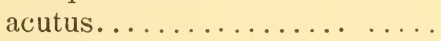

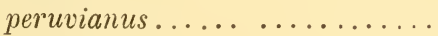

rupestris..............

Chroicocephalus atricilla.............

cirrhocephalus .........

cucullatus ...........

Franklini ............

Kittlitzi............

personatus............

Schimperi...........

serranus..............

Chrysobronchus leucorrhous. . . . . . . . .

Chrysomirus angustipennis............

prasinus................

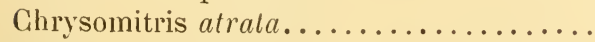


Chrysomitris columbiana............ Tomes III

magellanica............ III

Pages 51

Siemiradzkii............ III

uropygialis.............

50

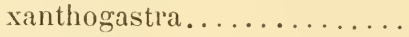

III

51

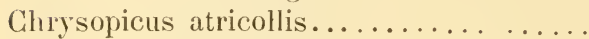

III

51

peruvianus..............

III

86

III

Chrysoptilus atricollis...............

punctigularis...........

III

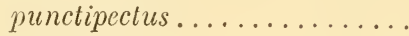

III

86

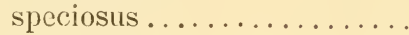

III

86

88

III

88

Ghrysothraupis chrysotis............

frugilegus..........

II

88

Parzudakii...........

II

475

488

II

pulchra ............

xanthocephala

474

462

II

II

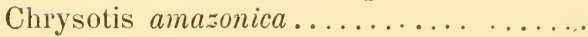

III

476

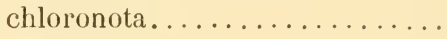

III

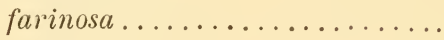

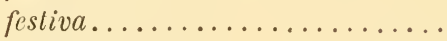

III

223

222

220

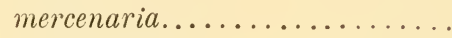

III

222

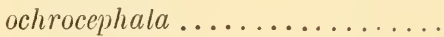

221

pœecilorhynchus............

III

224

III

III

225

Chrysuronia caruleicapilla............

Josephinx.............

Ciccaba cayennensis ...............

melanonola.................

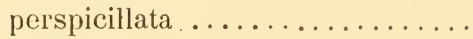

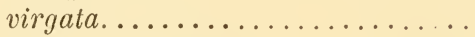

Cichlocolaptes adspersus.............

ochroblepharus..........

Ciconia jabirı..................

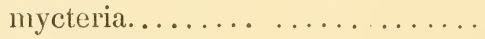

Cillurus bifasciatus..............

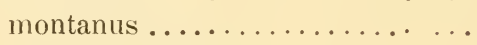

nigrofumosus. ............

palliatus. ...............

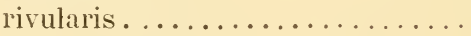

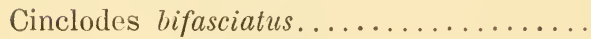

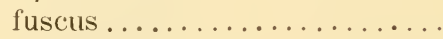

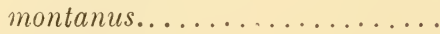

nigrofumosus .............

palliatus.................

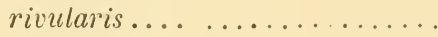

Cinclus leucocephalus................

morinellus................

Cinnicerthia peruana...............

peruviana.

I

409

409

$18 ?$

184

180

18?

157

157

411

411

111

108

110

109

112 
Circä̈tus solitarius. . . . . . . . . . . Tomes I

Circus albicollis................. I

campestris............. . . I

cinereus................ I

histrionicus.... $\ldots \ldots \ldots \ldots \ldots$ I

leucocephalus............. I

leucophrys.............. I

macropterus.............. I

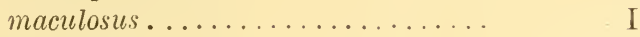

megaspilus .............. I

poliopterus.............. I

rufulus ................ I

superciliosus............. I

Cirripipra filicauda............... II

Cirrhocephalus plumbeiceps .......... III

Cissopis medius.................. II

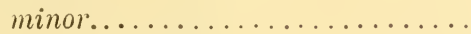

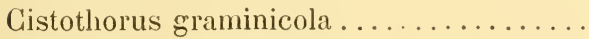

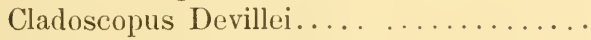

Claïs Guimeti.....................

Climacocercus concentricus............

Clypeicterus Oseryi...............

Clytolæma aurescens ................

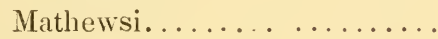

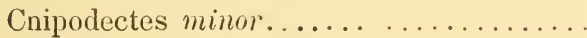

subbrunneus..............

Cnipolegus anthracinus.............

Coccoborus aureiventris.............

chrysograster .............

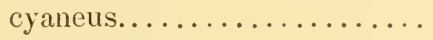

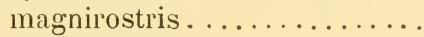

torridus................

Coccothraustes cyanea.............

Coccyzus cayanus.................

cinereus................

erythrophthalmus... . . ......

melanocoryphus...............

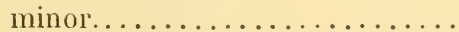

minutus. ...............

seniculus. ...............

rutilus .................

Cochlearius fuscus..............

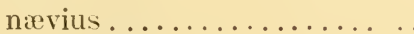

Coligena Warszewiczi..............

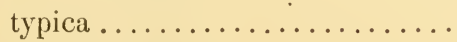

Gœophleus lineatus...............

mesochrysus .............

Cœreba atricapilla..................
Pages 129

169

$17 \mathrm{I}$

171

171

111

169

169

169

169

171

110

169

336

445

536,538

536,538

$5: 27$

175

364

162

402

387

295

294

294

208

III

III

III

III

III

III

III

III

III

III

III

III

III

III

III

III

I

I

III

III

I 
carneipes............... I

Pages 437

cærulea............... I

437

437

cyanea................. I

436

eximia............... I

437

melanocephala............ . . I

435

nitida................ I

Cola tixera ................... I

438,439

Colaptes puna................ III

137

rupicola............... III

Stol:manni............... III

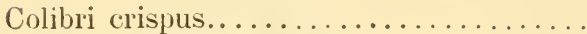

hirundinaceus ............. I

304

Colopterus galeatus............... II

229

Columba albilineata.................

III

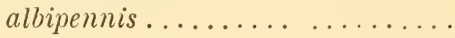

III

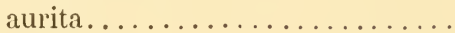

III

232

232

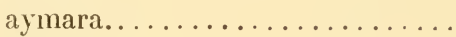

III

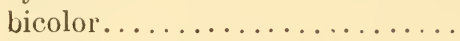

III

cinerea................

III

cruziana .................

III

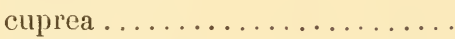

III

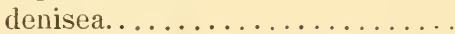

III

erythrothorax.

III

232

fasciata $\ldots \ldots \ldots \ldots \ldots \ldots \ldots$

III

249

frenata $\ldots \ldots \ldots \ldots \ldots \ldots \ldots \ldots$.

III

frontalis.................

III

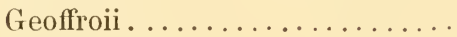

III

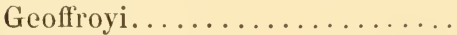

III

232

257

25 '

250

250

godofrida................

III

250

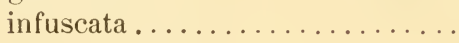

III

jamaïcensis. ..............

III

locutrix . . . . . . . . . . . . .

maculata .................

III

III

maculosa..............

melancholica ...............

melanoptera...............

meloda................. .

minuta................

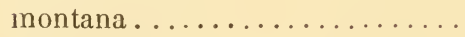

monticola ................

passerina. ...............

plumbea.................

rufaxilla...............

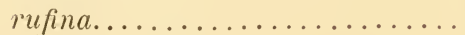

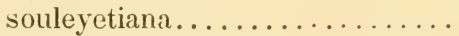


Columba subrinacea............... Tomes III

talpacoti................ III

P'ages 236

trifasciatal ............. III

246

vinacea .................. III

250

Columbina griscola............... III

$2: 35,236$

241

melanopteri............ III

239

Columbula erythothorax .......... III

$? \cdot 19$

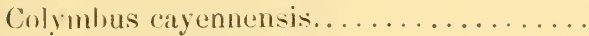

III

$49 \%$

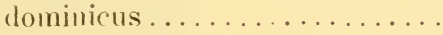

III

19.)

fulic:a...................

III

190

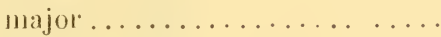

III

19:

Cometes caroli.

phaon.................

$33 i$

336

18'

Compsocoma sumptuosa.............

Compsothlypis inornata.............

Comptostoma flaviventre.............

Gonirostrum atrocyaneum............

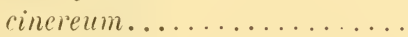

ceruleifrons............

cyaneum................

ferrugineicentre..........

Haviventre... . . . . . . . . . . .

sitticolor.................

(onopias cinchoneti................

Conopophaga ardesiaca..............

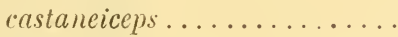

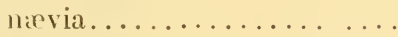

peruviana...............

Theresce...............

torrida.................

Conothraupis speculigera.............

Contopus ardesiacus................

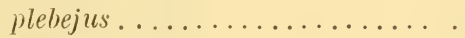

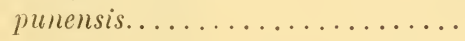

virens....................

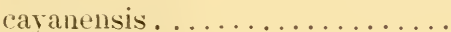

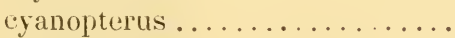

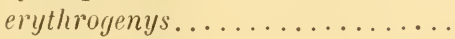

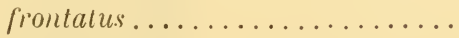

III

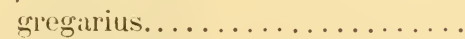

griseocephalus.............

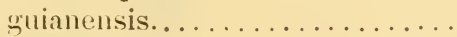

jugularis.................

III

Illigeri..................

III 
Conurus Luciani................ Tomes III

melanurus................ III

mitralus................ III

Pages 203

II

202

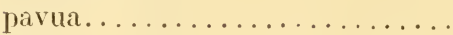

III

propinquus..............

III

196

roseifrons................

III

199

rubrifrons...............

III

rubrolarvatus.

III

rupicola.................

III

199

sitophagus . ..............

III

20 '

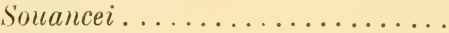

III

Weddelli...............

III

xanthopterus ..............

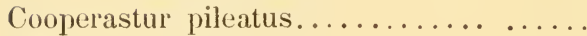

Coprotretes Jelskii................

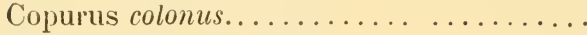

Goracina cephaloptera.. ............

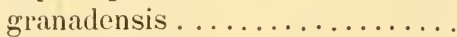

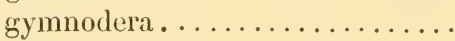

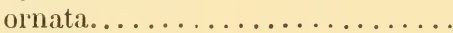

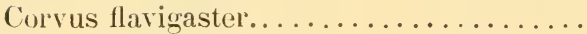

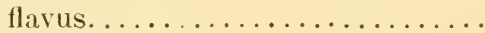

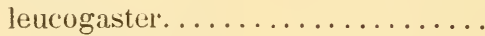

mystacalis. ..............

nudicollis. . . . . . . . . . . . . .

nudus..................

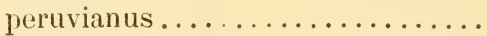

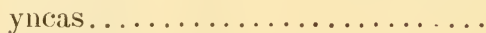

Corydalla chilensis

III

205

198

201

210

200

200

207

166

106

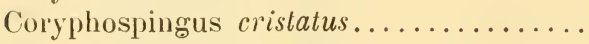

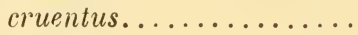

pileatus...............

Gorythopis anthoides................

anthoïdes humivagan.........

humivagans..............

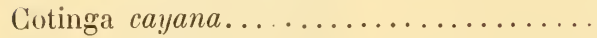

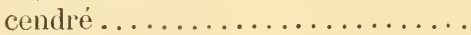

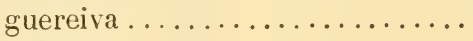

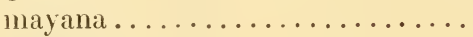

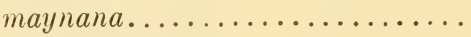

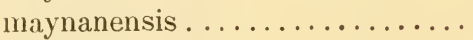

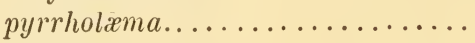

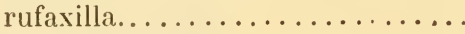

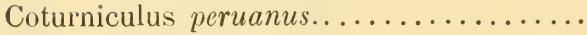

Cotyle flavigastra................

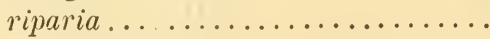

uropygialis ................

Craugasus flavigula. 
Crax alector.

Tomes III

rumanensis................ III

galeata.................. III

globicera..................

globulosa .................. III

mitu................... III

pauxi ................. III

Temmincki............... III

tuberosa ................. . . III

urumutum................. III

Craxirex pennsilvanicus............. I

Creagrus furcatus................. III

Creurgops verticalis................ II

Crex facialis................... III

femoralis. .................. III

galeata..................

Crithagra brasiliensis............... III

brevirostris . ............. III

chloropsis............... .

flaveola....................

Pentlandi .

Crotophaga americ'ına.

$a n i \ldots \ldots \ldots \ldots \ldots \ldots$

Casassi .................

levirostra................

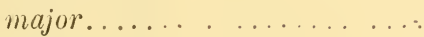

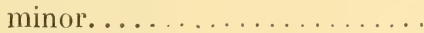

rugirostra . . . . . . . . . . .

semisulcata..............

sulcirostris................

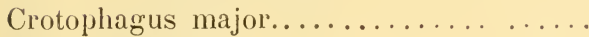

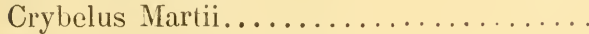

Crypticus platyrrhynchus............

Crypturus adspersus...............

atrocapillus..............

Balstoni...................

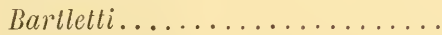

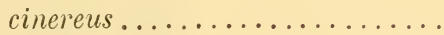

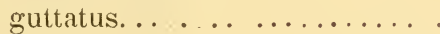

Kleei ..................

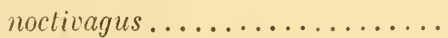

obscurus.................

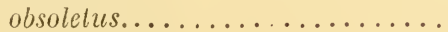

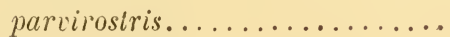

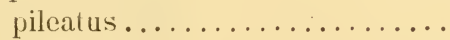

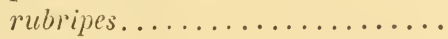

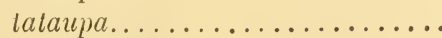

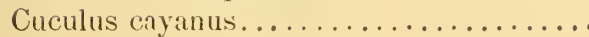

Pages 514

276

$: 666^{\circ}$

264

204

$266 ;$

266

511

267

265.

113

15.

501

321

326

327

55

61

56

55

58

181

180,181

181

180

180

180

180

181

181

180

111

113

299

294

299

301

300

995

$? 93$

302

$2 ! 1$;

291

298

298

303

297

$18 \tau, 188$ 
Cuculus cayennensis minor........... Tomes III

cayennensis nævius..........

erythrophthalmus............

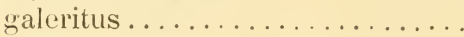

melanogaster ...............

III

181

monachus................

III

188

narius................

III

187

punctulatus................

III

ruficapillus ...............

III

rutilus ...................

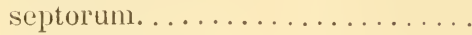

tenebrosus.................

Culicivora bilineata...................

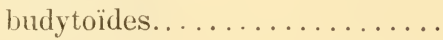

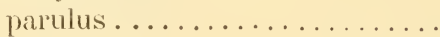

Cultrites Pucherani.................

Cranocitta jolixa..................

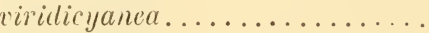

II

Cyanocorax mystacalis.

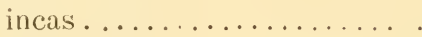

peruanus...............

violaceus................

virirlicyaneus. . ...........

398

396

396

399

400

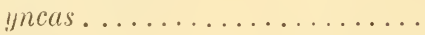

396

Cyamoloxia cyanca ................

Cyanomyia cyanicollis..............

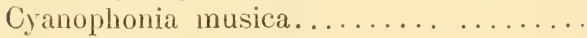

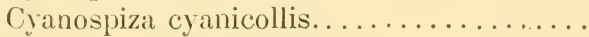

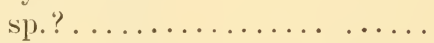

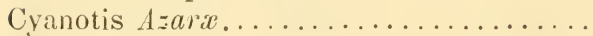

Cyanurus joliæa...................

Ciclorhis Contrerasi................

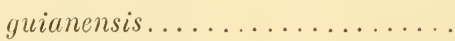

$$
\begin{aligned}
& \text { poliocephala............... }
\end{aligned}
$$

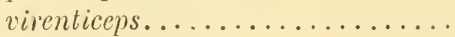

Grclorhynchus fulvipectus............

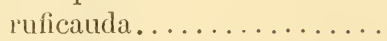

sulphurescens ..........

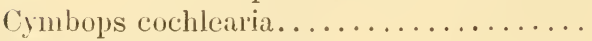

Cymbilaimus lineatus...............

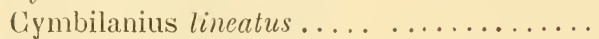

Cymindis cayennensis. .............

buteonides..............

megarhynchus ............

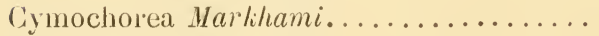

Gynanthus bifurcatus...............
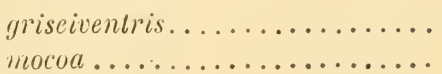
Cynanthus smaragdicaudus .......... Tomes I

Cyphorhinus griseicollis............ I

Pages 335

leucostictus............. I

512

513

marginatus........... I.

511

modulator............. I

508

phieocephishıs.......... I

508

prostheleurus .......... I

$51:$

Salvini............. I 509

lhoracicus.............. I

507

Cyphos macrodactylus.

128

macrorhynchus............ II

124

232

Cypseloïdes fumigatus............. I

232

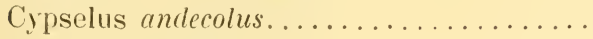

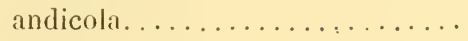

232

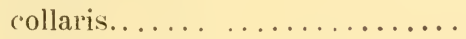

231

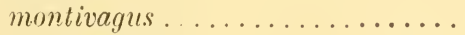

233

pelasgius............. I

$\rightarrow 90$

poliourus. .............. I

squamatus. . ............ I

229

234

D

I)acnidea albiventris ............. II

leucogastra............ II

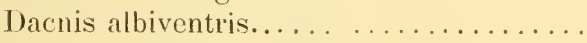

analis................... I

angelica..................

atricapillus.............. I

435

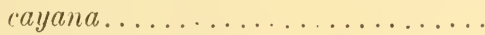

428

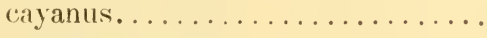

flurivenler......................

flaviventris.............. I

melanotis.............. I

modesta...................

pulcherrima ............. I

Dafila bahamensis............... III

ciesioscapulata............ [I]

pyrrhogaster.............. III

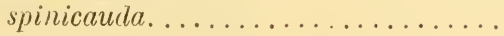

urophasianus ............. III 
Dasycephala thamnophiloïles.......... Tomes II

Dendrocincla funigata ............ II

Pages 372

merula............... II

II

169

Dendrocolaptes chunchotambo..........

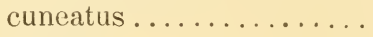

Devillei ..............

175

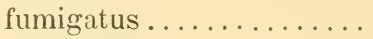

II

168

longirostris...........

171

merula...............

169

ocellatus .............

pallescens.............

radiolatus.............

170

Souleyeti.............

174

validus...............

169

Dendrocops fumigatus..............

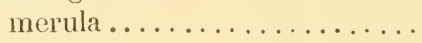

168

validus.................

Dendrocopus fuliginosus.............

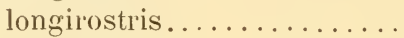

171

Dendrocygna autumnalis.............

469

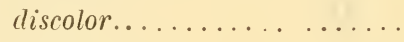

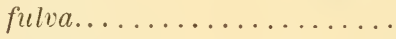

469

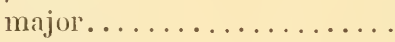

170

171

viduata................

471

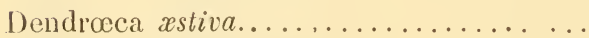

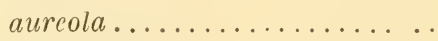

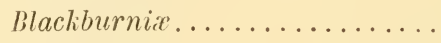

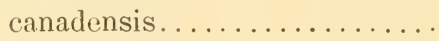

cxrulea ...................

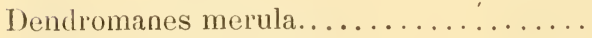

Dendrornis chunchotambo............

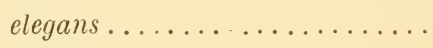

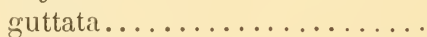

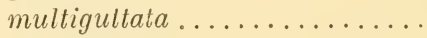

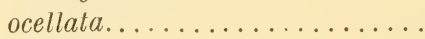

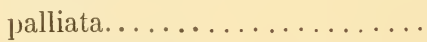

rosiripallens.............

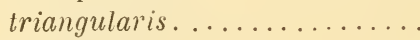

Dendroxetastes Devillei..............

Diallactes melanurus..............

transandeanus . ............

506

467

464

468

465

169

179

178

176

180

Diglossa albilatera.................

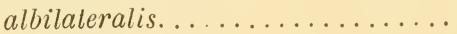

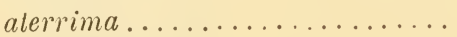

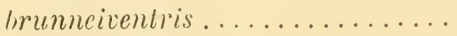

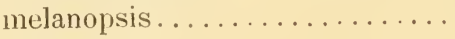

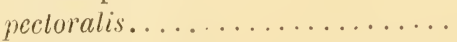


Diglossa sittoüdes............... Tomes I

Diglossopis cxrulescens............. I

Pages 417

Diodon bidentatus ............... I

$4: 2$

brasiliensis . . ........... I

157

157

Diomedea adusta ............... III

461

albatrus.............. III

461

exulans............... III

irrorata. ............. III

sparlicea .............. III

461

ilil

461

Diphlogrena aurora..............

383

iris ............... I

Warszewiczi........... I

354

383

Diphlogene aurore.............. I

383

Diplopterus nxvius................

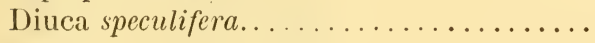

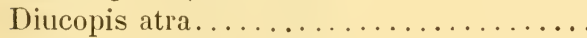

III

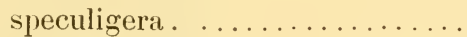

Diva albiventris.................

atrocxrulea...................

427

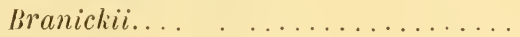

Vassori....................

II

456

II

II

455

454

Dives Warszewiczi.. .............. II

Dixiphia coracina............... II

433

Docimastes ensiferus ................

Schliephackei............

$34:$

375

375

Dolichonyx agripennis..............

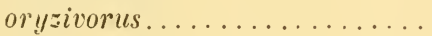

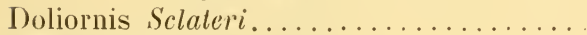

Donacobius atricapillus.............

brasiliensis..............

Doryfera Euphrosina ..............

Johannx.................

rectirostris................

502

.02

$28 \div$

285

$\because 81$

Doryphora Euphrosina. . . . . . . . ....

Johanna............... I

rectirostris. ........... I

Dryocopus albirostris . . . . . . . . . . III

linealus............ . II

Sclateri .............. . . III

trachelopyrus .......... III

Dryopicus lineatus

Dubusia lacrymosa. . . . . . . . . . . .

Dysithamnus ardesiacus. 
E

Egretta agami................. Tomes III

americana .............. III

Pages 396

leuce................. III

392

leucogastra...............

392

nivea.................

III

395

III

391

Elaenea miles......................

similis.................

876

II $\quad 276$

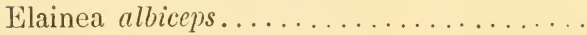

II

263, 261, 26j

albicollis...................... II

273

brevirostris..................

272

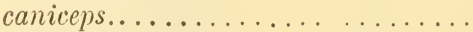

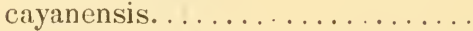

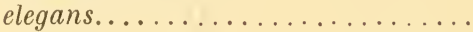

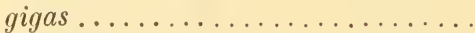

gracilis...................

leucospodia.................

lineata...................

luteiventris ................

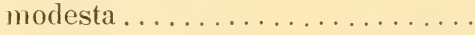

$\omega$

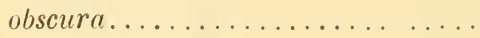

pagana ................. .

pallatang $x \ldots \ldots \ldots \ldots \ldots \ldots$

placens...................

subplacens.................

stictoptera................

Elainia viridiflara..................

Elanoïdes furcatus.. . ..............

$$
\text { yetapa.............. }
$$

Elanus torquatus....................

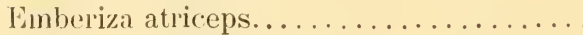

brasiliensis. . . . . . . . . . .

fulviceps..............

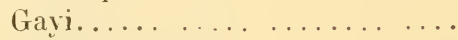

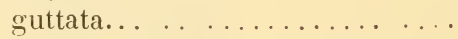

luctuosa . ..................

lutea....................

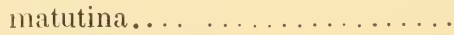

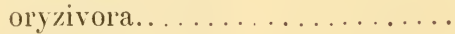

pileata.................

rustica.................

speculifera ...............

unicolor..................

uropygialis. .

II

II

269

II

275

II

266

II 265

II 971

II

267

II

351

278

263

270

$\because 62$

264

268

?68

201

261

137

137

1 i 0

III

III

31

II

III

III

III

III

III

II

III

III

III

III

III

II

$5 \%$

532

32

35

37

ifi

45

Embernagra brunneinucha............ 
Enpidagra brevirostris.

Pages :27:

Empidochanes fuscatus

313 olivus............... II

parcilurus............. II

$31: 3$

314

Enıpidonax andinus.............. II

319

minimus............. II

315

Empidonomus varius............. II

326

Eos cervicalis ....................

$19 \%$

Ephialtes cristatus................

III

$18 \mathrm{~s}$

Eremita griseigularis................

270

Ereunetes petrificatus...............

$36:$

semipalmata.............

362

Eriocnemis affinis.................

396

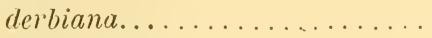

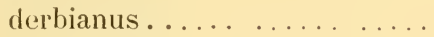

395

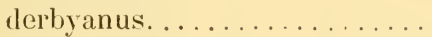

395

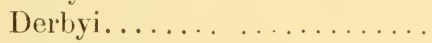

395

Dybowshii.

395

39 '

sapphiropygia ...........

397

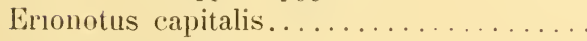

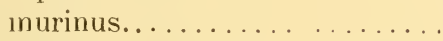

navius. . . . . . . . . . . .

Eriopus Derbyi...................

Érismatura cyanorhyncha.

ferruginea..............

leucogenjs .............

vittata. . . . . . . . . . . . . .

III

III

486

isi)

106

Erythrocnema unicincta ............

III

1:i.i

Esparvero calzado............... I

Eubucco aurantiicollis.............. . III

Hartlaubi .............. ... III

Eucephala cxrulea...................

Euchloris Riefferi.

Eucinetus Barrabandi .............. III

histrio.............. III

Euclosia Gayi .................

Eucometis penicillat $九 . . . \ldots \ldots \ldots \ldots \ldots$ II

Eudocimus albus.. ... . . . . . . . . . III

Eulidia Yarrelli.................. I

Eupetomena hirundo........... [

Euphona aureata................ II

Euphone chlorotica ................

Euphonia hicolor.............. II 
Euphonia chrysopasta............... Tomes II

Pages 448

crassirostris.............. II

44.5

hypoxantha........... II

445

melanura.............. II

147

mesochrysa............. III

510

minuta............... II

nigricollis............... II

440,442

olivacea ............... II

439

pumila................. II

442

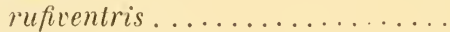

142

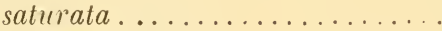

1.17

443

serrirostris............... II

440

stictifrons.............. II

442

xanthogastra............ II

414

Euprepiste boliviana .............. II

465

Eupsilostoma pusillum............... II

254

Eurypyga helias..................... III

388

Euscarthmus agilis................ . II

242

cinereus............. II

236

fulviceps $\ldots \ldots \ldots \ldots \ldots$ II

235

pileatus ...............

230

pyrrhops...............

232

reguloïdes............. . . II

241

ruficeps ............. II

235

rufigularis............ II

231

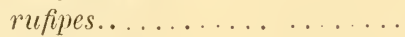

233

spicifer...............

squamicristatus..........

Wuchereri.................

Euschemon cyanolirma .............

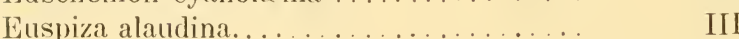

299

230

$\begin{array}{ll}\text { II } & 233 \\ \text { II } & 163\end{array}$

III 3.

II 532

fulviceps............... . II

468

479

$\therefore 59$

259

259

Condaminii

II

Euthraupis analis...............

Eutoxeres Condaminei................

\section{$\mathbf{F}$}

Falcinellus Ordi ... .............. III

Ridgwayi ............ III

Falco aguia ................... I

allifrons ................

albigularis................

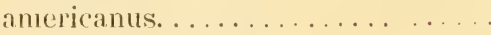

anatum . . . . . . . . . . . .

aquilinus 
Falco arundinaceus .............. Tomes I

Pages 127

aterrimus ............... I

103

aurantius . . . . . . . I

Beskii.................. I

149,150

bidentatus. .................

brachypterus . . . ............... I

160

brasiliensis.............. I

busarellus. . . . . . . . . . . . . I

cachinnans $\therefore \ldots \ldots \ldots \ldots \ldots \ldots$ I

calquin................. I

caracca ................ I

carolinensis.............. I

Cassini................. I

cayennensis .............. I

cinnamominus............. . I

127,144

coronatus ................ . . I

cristatus ............... I

crotaphagus............. I

cucullatus................ I

degener................. I

deiroleucus .................. I

delicatus ............... I

destructor............... . . I

femoralis................ I

formosus............... I

furcatus................. I

fusco-carulescens .......... . I

gracilis................ I

guianensis.... . . . . . . . . . . . I

halixetus................. I

harpyia................ I

Harrisii................ . . I

histrionicus................. . I

insectivorus . . . . ......... . I

Jacquini... . . . . . . . . . . .

latissimus . . . . . . . . . . . .

leucomelas................. I

leucorrbous .............. $\quad \ldots$

magnirostris............... I

Mauduyti................ [

melanobronchus .. . ........ I

meridionalis............... I

nigriceps...............

nigricollis.................

nisus ..................

nudicollis .................

ornatus . 
Falco palliatus .................. Tomes I

palustris ............... [

Pages 144

pennsylvanicus............ I

169

113

percontator................ I I

160

pileatus................ I

166

plancus................. I

92

plumbeus............... I

138

regalis............... I

131

rufifrons ................ I

140

rufigularis. . ............ . . I

150

rutilans . . . . . . . . . . . . . I

110

Sonnini................ I

133

sparverius................ I

154

superbus................. .

135

tharus......................

thoracicus. ................. I

tyrannus................. I

unicinctus................ . I

urubitinga. .............. I

Wilsoni................. I

zonurus............... I

Faxado ..................... I

150,151

134

106

10 '

113

101

160

393

391

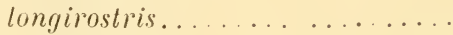

297

Florisuga flabellifera ...............

997

mellivora...............

Fluvicola albiventris............. II

205

atripennis . ............ II

206

bicolor . ............... II

205

onanthodis............ II

192

pica.................. II

205

rufipectoralis ............ II

194

Formicarius analis................. II

553

errthropterus . ......... II

lugubris ............... II

nigrifrons.............

nigromaculatus. ..........

Formiciora ardesiaca..............

bicolor .............. II

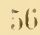

crerulescens ...........

callinota..............

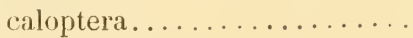

(inerascens............ II

hæmatonota...........

IIauxwelli...............

Menetriesii 
Formicivora pygma.............. Tomes IL

quadrivittata.......... II

Pages 37

quixensis............. II

38

rufatra............ II

51

l'ufimaıginatı......... II

49

Fregata aquila.................. III

$3 \cdot \hat{\prime}$

Fringilla alaudina............... III

araguira..............

III

arvensis...............

III

aureiventris . . . . . . . . . .

III

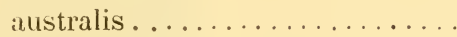

III

brasiliensis............. III

campestris ............. . III

i)

28

61

capensis . . . . . . . . . . . .

III

chilensis ................

III

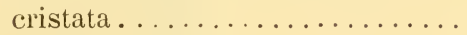

III

cucullata ................

III

cyanomelas...............

I

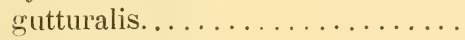

III

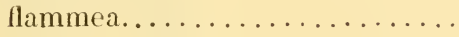

III

flava................. III

flaveola...................

III

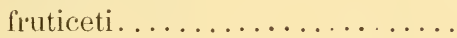

III

Gayi................ III

luteiventris.............. III

melanocephala............ III

nitens................. III

pileata............... III

III

splendens...............

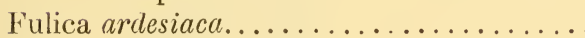

III

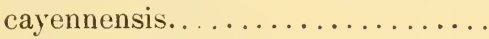

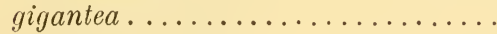

Fuligula Nationi.................

III

III

III

Furnarius cinnamomeus.............. .

cunicularius ..............

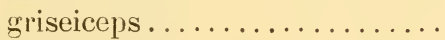

leucopus.................

longirostris...............

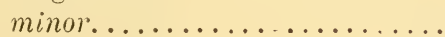

torridus.................

II

II

II

II

II

II

II

4.5

55

35

45

45

28 
Galbula chalcoptera............... Tomes III

chalcothorax.............. III

Pages 1:0

cyanescens.............. III

118

flavirostris ..............

III

115

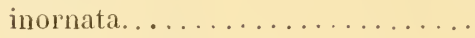

116

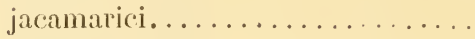

120

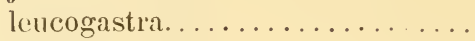

III

122

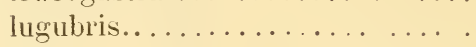

III

III

118

tombacea ..................

III

120

III

115

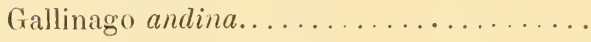

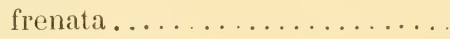

III

37.5

Jamesoni....................

III

375

III

376

Gallinula carolina..................

III

cayanensis ............. III

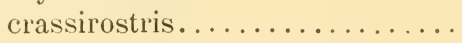

III

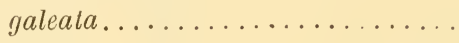

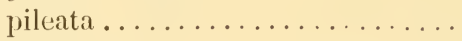

plumbea...............

III

III

320

318

$3: 6$

327

323

III

319

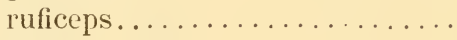

III

318

saracura.............. III

Gambetta flavipes............... III

319

melanoleuca ...............

Gampsonyx Swainsoni..............

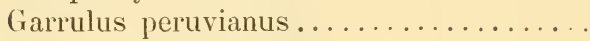

viridicyaneus.............

III

366

140

396

II

400

III

Garzetta candidissima ..............

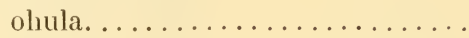

III

393

393

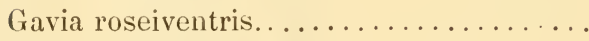

III

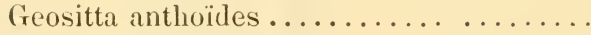

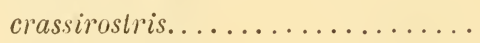

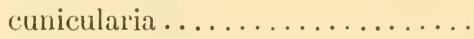

II

cunicularia juninensis..........

Frobeni..................

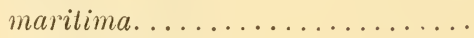

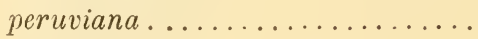

II

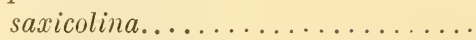

II

tenuirostris................

Geothlypis aquinoctialis.............

aquinoctialis peruviana...... .

auricularis...............

poliocephala.............

velata ..................

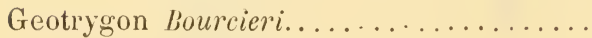

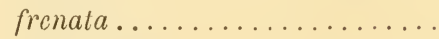

montana................

Geranoaëtos aguia

melanoleucus.............

Geranospiza cirulescens.

II 
Geronticus cayanensis............. Tomes III

Glareola cuneicauda ................ III

Pages 4:0

Glaucidlum ferox.................

Glaucis aflinis.

rervinicauda................

260

hirsuta.................

201

hirsutus...............

261

mazeppa ..............

261

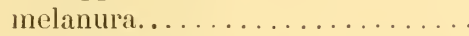

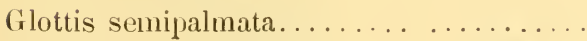

Glyphidiura perlata ...............

261

364

Glyphorhynchus Castelnaudi............

Gnathospiza Raimondii...............

Golondrina domestica...............

Gouldia Langsdor $f i \ldots \ldots \ldots \ldots \ldots \ldots$

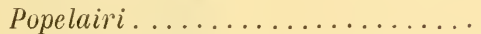

302

Popelairii................

Gracula chrysoptera............... 416

fotida...................

390

longirostris...............

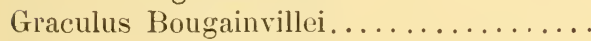

brasilianus ................

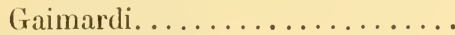

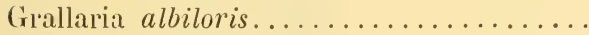

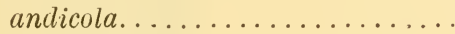

brevicauda .................

brevicaula minor.............

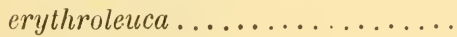

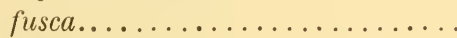

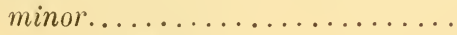

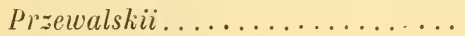

regulus.................

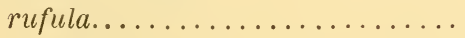

squamigera...............

Grus psophia.

Grypus Vieilloti .... .............

Guiraca cinerea....................

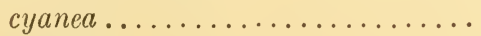

cyanoïdes ................

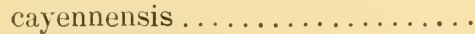

III 
(iymmopelia erythrothorax........... Tomes III

Gymnops aterrimus............... I

Pagres 249

103

strigillatus............ I

Gypagus condor............... I

gryphus............... I

papa............... I 81

(iyparchus papa...............

Gyrinorhynchus rufirostris. . ....... II

Gyrola gyrolö̈les.............. II 40 ,

H

Hadrostomus audax............... II

356

homochrous............ II

$35 \%$

minor.............. II

358

II:mophila Stolzmanni.............

Hrematopus areticus................

III

351

aler................. III

351

brasiliensis . . . . . . . . . .

III

351

ostralegus ...............

III

palliatus.................

III

351

Townsendi ..............

III

350

Ialadroma Berardi ................

III

351

Garnoti .................

III

166

165

Haliätus erythronotus.............

melanoleucus .............

115

$1: 4$

Halicus albigula.................

brasilianus................

429

Gaimardi.............. III

431

46$.

IIalodroma Garnoti .................

III

Hapalocercus acutipennis..............

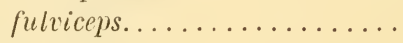

Harpagus bidentatus................

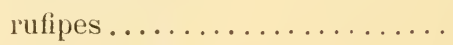

II

Harpiprion cxrulescens................

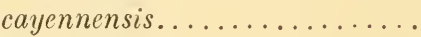

IIarpy haliaëtus coronatus..............

IIarpyia bracchata ................

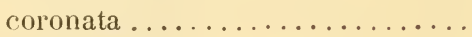

destructor.................

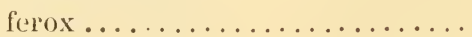

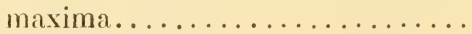

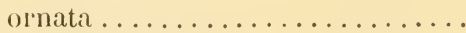

IIeliangelus amethysticollis............

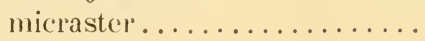

viola $\ldots \ldots \ldots \ldots \ldots \ldots \ldots$.

Helianthea aurora................. 
Helianthea cupripennis............. Tomes I

dichroura.............. I

iris................ I

osculans............... I

Helias phalænoïdes............... III

Heliochera rubrocristata............ II

rufaxilla............... II

Pages 340

377

381

376

388

381

383

Heliodoxa Leadbeateri.............. I

287

otero............... I

287

Heliomaster albicrissa............. I

393

longirostris..............

391

pallidiceps..............

392

Sclateri................

392

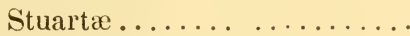

391

Heliomastes albicrissa.............

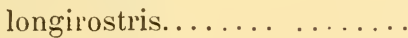

Heliothrix auritus...............

longirostris. ........... I

Heliornis fulica.................

fulicarius..............

Heliotrypha micraster.............

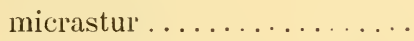

viola..................

Helymus micraster................

III

490

490

380

380

379

380

Hemidacnis albiventris.

427

Hemipalama himantopus ............

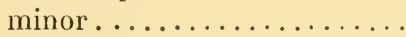

multistriata .............

363

III

III

362

III

363

Hemipipo Tschudii...............

II

334

232

231

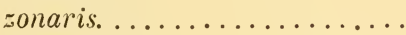

Hemispingus auricularis. ............

superciliaris ...........

517

285

285

Johannæ.............

rectirostris............

284

Hemithraupis flavicollis .............

511

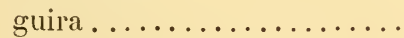

melanoxantha..........

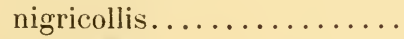

Henicorhina leucophrys..............

leucosticta..............

Herodias cærulea................

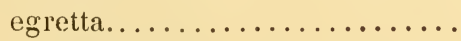

leucogaster..............

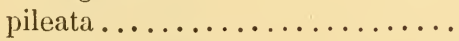

Herpetotheres cachinnans. 
Herpsilochmus argentatus.......... Tomes II

axillaris.............. II

Pages j9

molacilloïdes............ II

35

puncticeps............ If

35

rufimarginalus.........

Heterocercus lineatus.

351

lintealus............. III

510

Heterocnemis argenlala..............

bicolor.

II

miarginatus... . ....................

510

511

Heteropelma Amazonum.............

Wallacii...............

350

351

Heterapygia Bonapartei

360

Heterospizias meridionalis............

Hiaticula Wilsoni..................

343

Himantopus mexicanus..............

nigricollis.............

Hirundinea bellicosa ..............

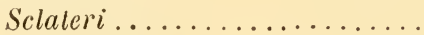

Hirundo requatorialis.............

albicollis................

albilinea................

albilineata ................

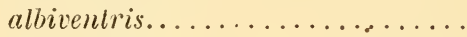

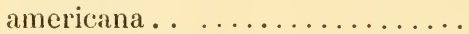

andecola ................. .

cahirica..................

cayanensis................

chalybaca ................

cinerea ...................

cærulea...................

collaris .................

cyanoleuca.................

erythrogastra...............

fasciata..................

flavigastra ...............

flaviventer ................

freti Hudsonis..............

hortensis . ...............

jugularis..................

kamtschatica...............

leucoptera .................

leucopygia.................

leucorrhoa..................

ludoviciana ................

melampyga...............

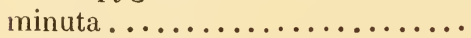

oxyura 
Hirundo pascuum................. Tomes

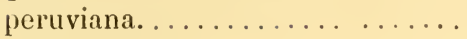

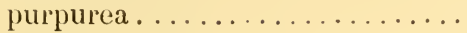

riparia.................

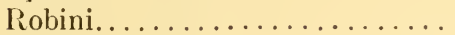

ruficollaris ...............

puficollis. . . .............

rustica saturata.............

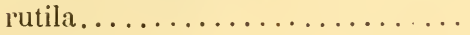

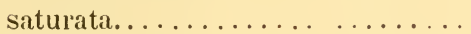

subis

tapera..................

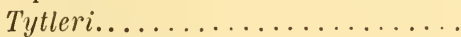

versicolor.................

violacea ................

zonaris

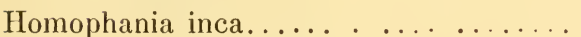

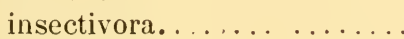

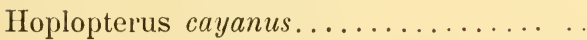

Hydropsalis climacocercus.............

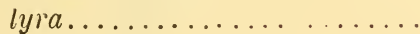

segmentala $\ldots \ldots \ldots \ldots \ldots \ldots$

trifurcata...............

trifurcatus. ...............

Hylocharis cyanea...............

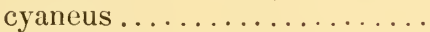

sapphirina...............

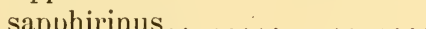

Hylolophus leucauchen. . ............

Hylophilus cæruleus ..............

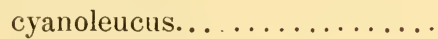

ferrugineifrons............

flaviventris...............

frontalis...............

olivaceus...............

Hypochrysia aurora................

Hypocnemis cantator.................
cantator peruvianus........

elegans...............

flavescens...............

hemileuca................

leucophrys.............

lepidonota .............

lugubris.................

melanolæma.............

melanosticta............

melanopogon.............

melanura.

Tones

Pages 236

243

236

245

$2: 9$

503

246

502

229

502

236

236

502

236

236

2.31

388

389

335

221

III

I 
Hypocnemis myolherina............. Tomes II Pages 65, III 509

nxvia................ II

pœcilonota............ II $\quad 64,75$

schistacea............. II $\quad$ II 69

subflava............... II $\quad 63$

Theresi............. II

Hypolia otero.................. I

Hypolophus leucauchen............. , II 15

Hypomorphnus ardesiacus........... I . 109

leucurus........... I $\quad 115$

rutilus ............. I 110

unicinctus........... I $\quad 106$

urubitinga............ I 104

IIypothlypis callophrys............ III $\quad 510$

iridina............... II 453

Hypotriorchis aurantius... . . $\ldots \ldots \ldots$. I $\quad 149,150,151$

deiroleucus............. I $\quad 149$

femoralis............. I 151

rufigularis............ I

Hypoxanthus brevirostris............ III $\quad 90$

Rivolii brevirostris........ III $\quad$ III

Hypsibamon andicola.............. II . 85

Ibiyau acanelado................. I $\quad$ I 219

Ibis alba.................... III 415

crerulescens ................ III 419

cayennensis................. III 420

dentirostris............... III 420

falcinellus.................. III 446

longirostris................ III

nandapou................... III 414

plumbea................... III 419

sylvatica.................. III 420

Ibycter anericanus................. I

aler...................... I 103

chimachima................ I $\quad$ I 99

$\begin{array}{ll}\text { chimango................... } & 97\end{array}$

megalopterus............... I 101

Icterus atrigularis................ II

cayanensis.................. II

chrysocephalus................ II $\quad$ II

chrysopterus ............... II 429

citrinus.................... II $\quad 426$

croconotus................... $\quad 4.90$

grace-annx................. II 419 
Icterus guianensis............. Tomes II

Hauxuelli................ III

Pages $4: 27$

icterocephalus ............. II

510

jamacai.................. II

425

mesomelas................. II

420

sericeus. $\ldots \ldots \ldots \ldots \ldots \ldots \ldots \ldots \ldots$ II

417

tanagrinus................ II

422

433

Ictinia plumbea............... I

138

Inca mystacalis.................. III

446

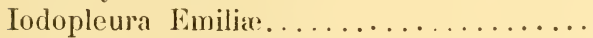

II

388

guttata.............. II

388

Isabellx............. II

388

Ipoborus montanus ................

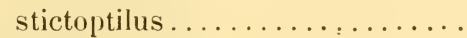

Iridornis analis.

Jelskii................. II

478

Reinhardli.............. II

$17 \%$

Iridosornis analis

Ischnoscelis crerulescens

Ispida americana viridis .

Ixothraupis chrysogaster ...........

Jacamaralcionides leucotis............

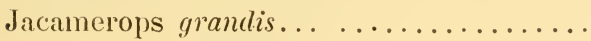

Isidori . . . . . . . . . . . . .

Јари пеgro..................

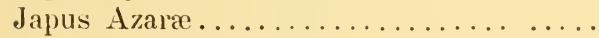

bursarius.................

Joliema Schreibersii................

\section{K}

Klais Guimeti................ I 
Lafresnaya Saulæ................ Tomes I

Lampornis iridescens............. I

Pages 283

mango ............. I

281

violicauda............. I

281

281

Lampraster Branickii............... I

286

Lamprolita platyrhyncha............ III

$12 \%$

Lampropsar guianensis.

433

tanagrinus.

433

Warszewiczi.............

433

390

Lampropygia cœligena ...............

$: 387$

Lamprotes albocristatus ............

Laniagra guianensis................

448

Lanio versicolor................. II

Lanius atricapillus............... II

500

365

cayanensis.............. II

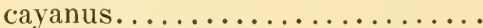

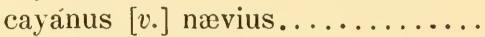

354

354

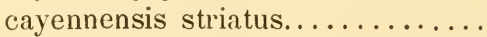

doliatus. ..................... II

funebris................ II

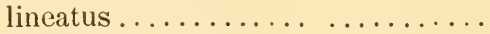

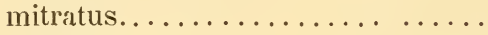

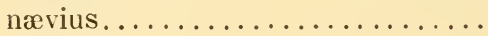

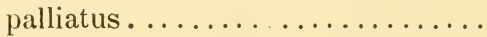

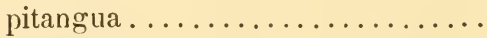

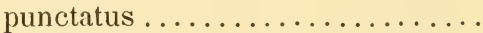

II

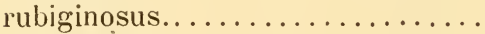

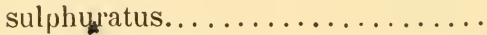

II

superciliosus ................

Larus albipennis.

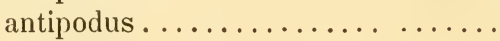

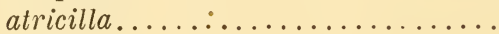

III

Azaræe.....................

III

III

Belcheri.................. III

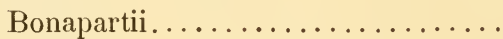

III

Bridgesi ...................

III

cirrhocephalus ................

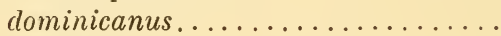

III

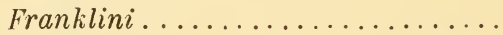

III

III

Fritzei ................... III

Frobeenii..................

III

Frobeni................. III

fuliginosus ...............

III

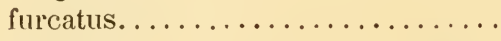

III

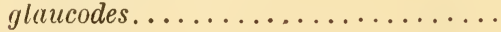

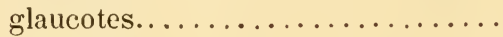

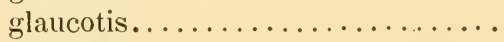

modestus. .................. 
Larus parasiticus ................ Tomes III

poliocephalus............. III

ridibundus . .............. III

Sabini.... $\ldots \ldots \ldots \ldots \ldots$ III

serranus................. III

Verreauxi... $\ldots \ldots \ldots \ldots \ldots$ III

Pages 458

vociferus... ............. III

4.50

456

452

II $\quad 447$

Lathria cineracea. . $\ldots \ldots \ldots \ldots \ldots \ldots \ldots \ldots \ldots$ III

447

368

cinerea............... III 368

Latirallus facialis. . . . . . . . . . . . III

324

Leadbeatera otero............... I

287

Warszewiczi .......... I

383

Legatus albicollis ............... II

จนว

Leistes albipes.... . . . . . . . . . . II

429

americanus............. II

427

guianensis............... II

427

Leobia caroli.................. I

$33 \pi$

Lepidonas speciosa............... III

931

Lepidothrix cyaneocephala. ........... II

34.3

Isidori.............. II

342

Leptasthenura xgilhaloüdes.............

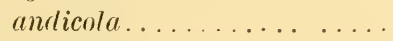

120

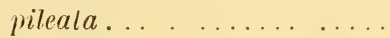

118

slriala .... II

119

121

Leptaxyura cinnamomea. ........... II

127

Leptodactylus Mitchelli............ III

372

Leptodon cayennensis... ... . . . .... I

144

megarhynchus

141

Leptopelecanus Molina.

I

424

Leptopogon amarocephalus...........

III

248

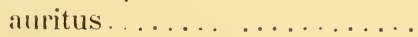

II

$\because 46$

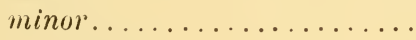

peruvianus.

$21 \%$

248

rufipectus..............

249

superciliaris............ . II

246

viridiflavus ............ II

261

Leptoptila ochroptera. .

.255

rufaxilla.............. III

Verreauxi ............. III

251,256

956

$37 ?$

Lерtopus.................. III

436

Lepturus.................. III

2.23

Lepturus brevicauda.. . .......... II

?35

fulviceps. ........... II

330

Lesbia gracilis.

nuna.............. I

Lessonia orcas ................ II

Lestris antaretica.............. III

458

antarcticus $[\ell.] \beta$ chilensis. 
Lestris pomarinus.............. Tomes III

Leưcippus chionogaster............. I

Pages 459

chlorocercus ............. I

400

nigrirostris............ I

399

pallidus................. I

viridicauda............... I

400

402

399

Leucocarbo Bougainvillei............. III

430

Leucolia cyanicollis.................

Pelzelni .................

397

397

Leucophæus Belcheri ............. III

448

Leucophaius modestus ..............

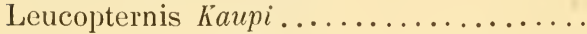

Kuhlii ...............

schistacea..............

superciliaris .............

Limosa adspersa. ...............

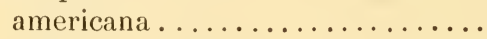

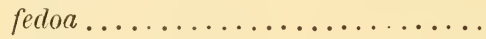

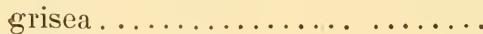

III

449

117

117

109

117

379

379

379

373

Linaria analis................. III

analoïdes .................

III

Lipaugus albogriseus.............. II

cineraceus................

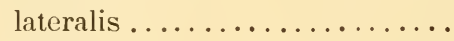

$\operatorname{simplex} \ldots \ldots \ldots \ldots \ldots \ldots \ldots \ldots$

virussu. . . . . . . . . . . .

Lipocentrus andinus $\ldots \ldots \ldots \ldots \ldots \ldots \ldots$ III

423

Lochmias obscurata..................

Loddigesia mirabilis .................

Loddigiornis mirabilis.............

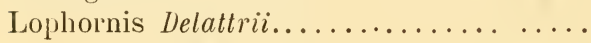

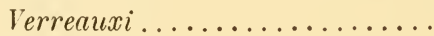

Lophospiza cruenta . . . . . . . . . . . .

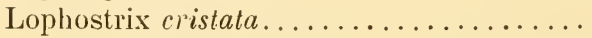

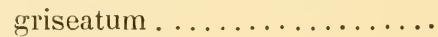

Lophotriccus spicifer..............

squamicristatus............

113

318

318

299

300

Loro cabeza amarilla .............. III

III

cyanea.$\ldots \ldots \ldots \ldots \ldots \ldots \ldots \ldots$ III

ignobilis ............... III

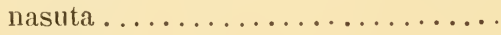

plebeja. . ................ III

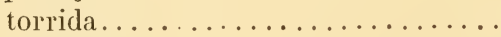

III

virginica $\ldots \ldots \ldots \ldots \ldots \ldots \ldots \ldots$

Lurocalis Nattereri................ .

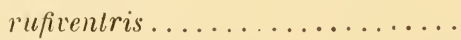

Lypornix rufa 
Lypornix ruficapilla

\section{M}

Macabra melanonota............. I

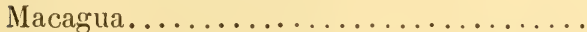

cachinnans ............. I

145

145

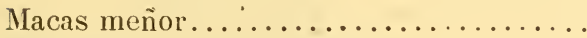

Machæeropterus pyrocephalus.......... striolatus.

Macrocercus ararauna...............

193

militaris.............. III

191

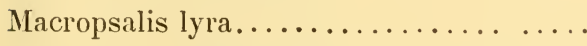

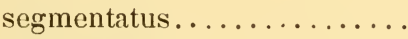

225

223

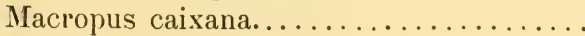
$18 \tau, \tilde{188}$

Macrorhamphus griseus...............

Malacocichla dryas..................

maculata .............

Malacoptila fulvogularis..............

Manacus

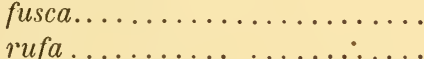

Edwardsi................ II

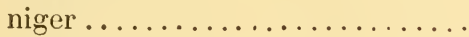

rubrocapillus ...............

Maracana Luciani..................

III

Weddelli............... III

Margarornis brunnescens. .............

Mecocerculus caloptera..............

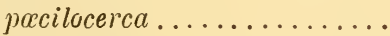
Megalophus Castelnaudi..............

Megapicus albirostris...............

hæmatogaster ............

Sclateri.................

III

trachelopyrus . ...........

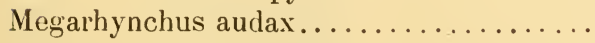

Melampicus hirundinaceus. . .........

Melanerpes cruentatus.............. 
Melanodiglossa albilatera............. Tomes I aterrima............. I

Pages 418

419

Melanomitris atrata.............. III

53

uropygialis ........... III

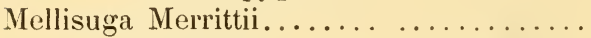

ruficeps.................

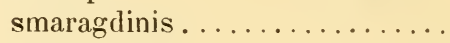

surinamensis.

334

297

Melopelia meloda................ III

Merganetta columbiana.............. III

leucogenys...............

Turneri.............. III

486

Merula chiguanco ..................

486, 488

488

494

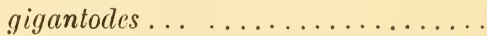

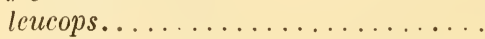

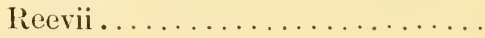

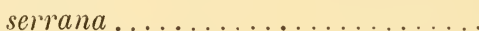

Mesopicus fumigatus.

495

496

490

4.6

Netallura æneicauda. .

cupreicauda............. .

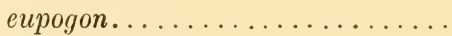

Hedviga.................

Jclskii ...............

ораса...................

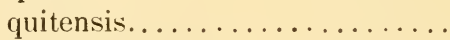

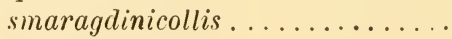

tyrianthina..............

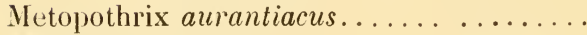

Metriopelia aymara................

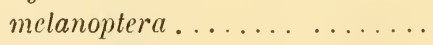

Micrastur concentricus. . ...........

dynastes.................

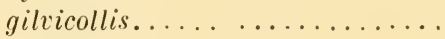

guerilla.................

macrorhynchus....... .....

Mirandollei.................

Pelzelni..................

semitorqualus...............

Microcerculus bicolor. .............

leucostictus............

marginatus.............

Microchelidon tibialis...............

Micropogon anızonicus. ...........

flavicollis................

Micropogon glaucogularis..........

Micropus andicola.... ............

Microrhopias callinota............. 
Microrhopias quixensis............. Tomes II

Microsittace Luciani...... . . . . . . . . . III

rupicola ............ III

Souancei............. III

Microspingus trifasciatus............. II

Milvago chimachina...............

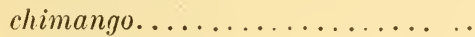

megalopterus................

ochrocephalus ..............

Milvulus tyrannus ...............

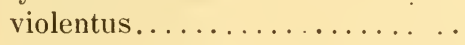

Milvus cenchris.................

furcatus.................

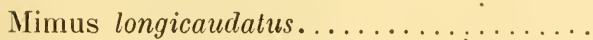

thilius.....................

Mionectes oleagineus................

poliocephala..............

striaticollis ................

Mitrephorus ochraceiventris...........

Mitu braziliensis..................

Mitua tuberosa ..................

Molothrus purpurascens..............

Molybdophanes cæerulescens ...........

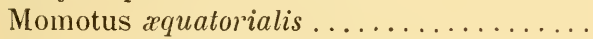

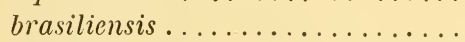

Dombeyi................

Martii...................

microstephanus..............

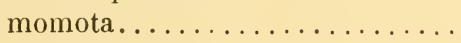

platyrhynchus ............

ruficapillus...............

semirufus . . . . . . . . . . . .

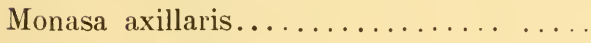

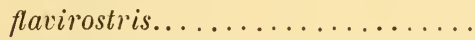

nigrifrons ................

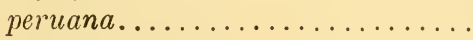

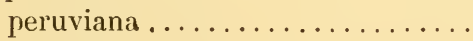

tenebrio..................

tenebrosa ...............

unitorques................

Morinella collaris

II

I

I

I

II

II

Morphnus cristatus ...............

harpyia .............. 
Motacilla canadensis .............. Tomes III

cayana................ I

chrysocephala............ I

cyanocephala............ I

flavicauda .............. III

Pages 506

428

guira ................ II

magellanica ............. I

464

428

507

multicolor. ..............

510

multicolor.................

III

529

rubiginosa............... III

507

tricolora..................

506

507

Nouette à queue fourchue............

III

457

Mulsantia mirabilis................

III

318

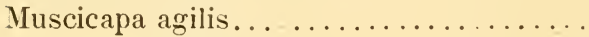

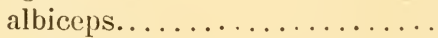

II

263

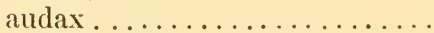

II

289

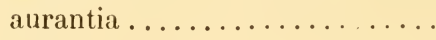

II

360

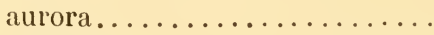

II

321

barbata................

II

298

bicolor...................

205

bimaculata..............

313

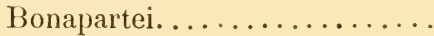

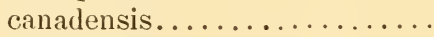

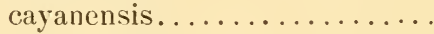

chloronotus . . ...........

cinerascens .............

cinerea .................

colon...................

colonus..................

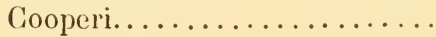

coronata................

cruenta.................

468

468

275

245

370

468

209

209

316

307

389

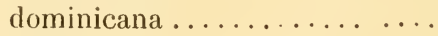

$: 07$

360

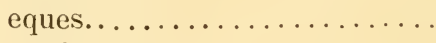

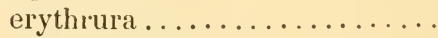

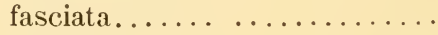

filicauda ...............

flammiceps..............

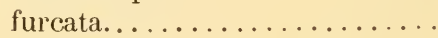

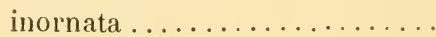

legatus ..............

leucocephala .............

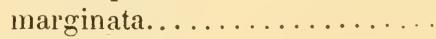

megacephala .............

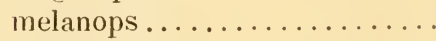

melanoxantha .

301

305

209

305

II

327

II

316

II

273

Il

II

207,275

364

283

II

506

II

225

nævia .................

II

305

nigriceps.... . . . . . . .

oleaginea ...............

359

94 


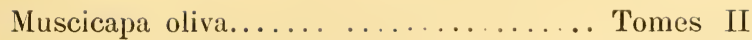

olivacea .............. I

Pages 313

pagana.............. II

443

parulus ............... . II

262

phanoleuca,..............

239

pica ..................

II

332

plumbea...............

205

porphyrobroncha $\ldots \ldots \ldots \ldots$

368

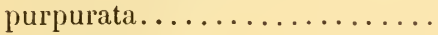

389

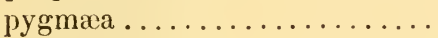

389

rubinea ................

37

rubra.................

307

rubricollis...............

394

rufina..................

389

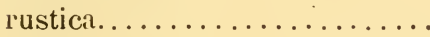

ruticilla................

370

similis................

507

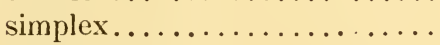

276

370

striaticollis. ..............

sulphurea..............

277

thamnophiloïdes..........

372

trivirgata ...............

tyrannulus..............

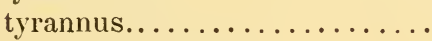

tyrannus brasiliensis. ........

virens..................

virgata $\ldots \ldots \ldots \ldots \ldots \ldots \ldots$

vociferans................

Muscicapara oleaginea...............

striaticollis ............

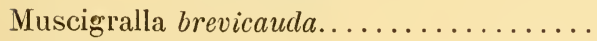

Muscipeta albiceps.................

albicollis..............

armillata ...............

cayennensis .............

chloronotus...............

cinnamomea..............

citrina ..................

Guillemini. ..............

leucocilla. ...............

monarcha ..............

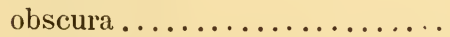

ralloïdes................

ruficauda..................

Vieilloti................

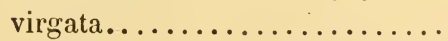

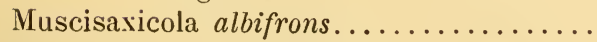

albimentum. 
Muscisaxicola cinerea ................. Tomes II

flavinucha............. II

Pages 21?

flavivertex........... II

211

fluviatilis...............

211

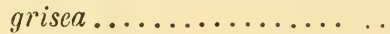

220

juninensis...............

213

maculirostris............

214

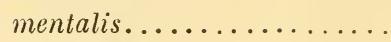

219

rubricapilla.............

218

rufipennis..............

215

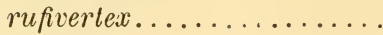

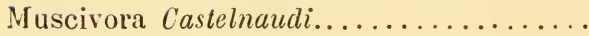

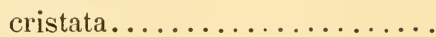

$2: 1$

216

295

II

295

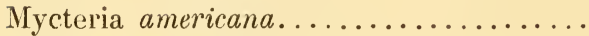

II

411

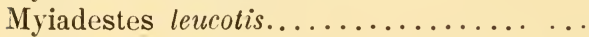

ralloïdes................

Myiarchus atropurpureus............

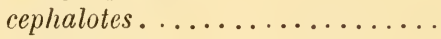

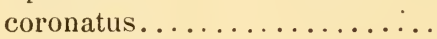

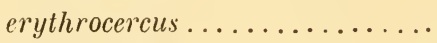

fasciatus. ..............

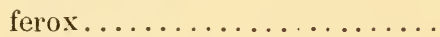

ferrugineus . ............

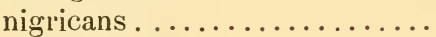

nigriceps...............

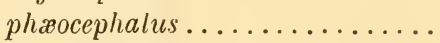

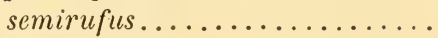

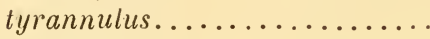

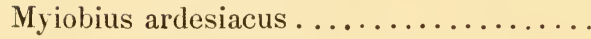

aureiventris .............

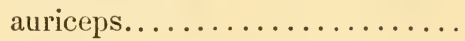

barbatus..................

borealis................

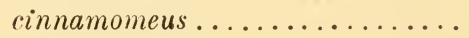

crypterythrus .............

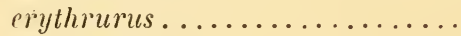

luteoventer................

navius ..................

Nationi..................

nigricapillus.............

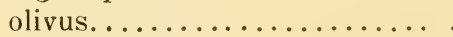

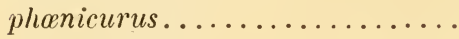

pyrrhopterus..............

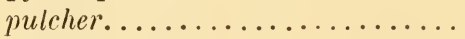

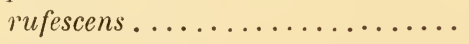

sulphureus ..............

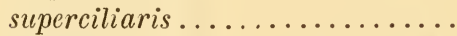

villosus..................

III

455

$45 \cdot 1$

311

322

310

$3: 0$

208

322

297

204

324

323

325

321

317

30 ?

305

298

316

299

304

301

290

305

303

$25 \pi$

313

30 ?

300

30 ?

303

277

306

II

II

จ99 
Myiobius virens.............. Tomes II

xanthopygius............ II

Pages 318

Myioborus melanocephalus............

verticalis.............. I

481

Myiodioctes canadensis. ............ I

468

tristriatus ........... I

472

Myiodynastes atrifrons............. II

291

audax............... II

289

chrysocephalus.......... II

293

luteiventris............ II

290

luteoventer........... II

290

solitarius.............. II

288

Myiopatis pusilla.

255

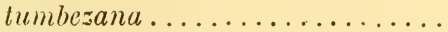

252

Wagx ................ II

253

Myiophobus crypterythrus...........

304

pœecilurus ............ II

Myiothera analis

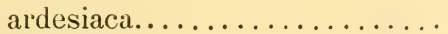

fuliginosa .............

nigrimaculata............

rufimarginata............

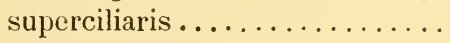

thamnophiloïdes...........

Myiotheretes erythropygius............

striaticollis............

Myiothlypis luteoviridis

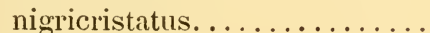

Myioturdus fuscater. ...............

Myiozeta cayennensis...............

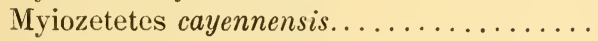

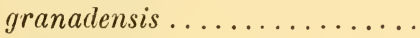

luteiventris..............

similis................

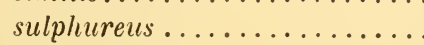

Myrmeciza argentata ..............

hemimelana...............

leucaspis..............

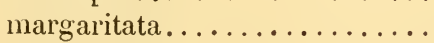

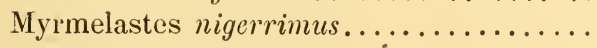

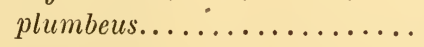

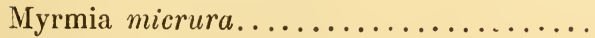

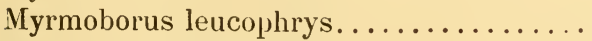

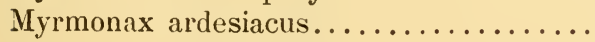

leucophrys .............. 


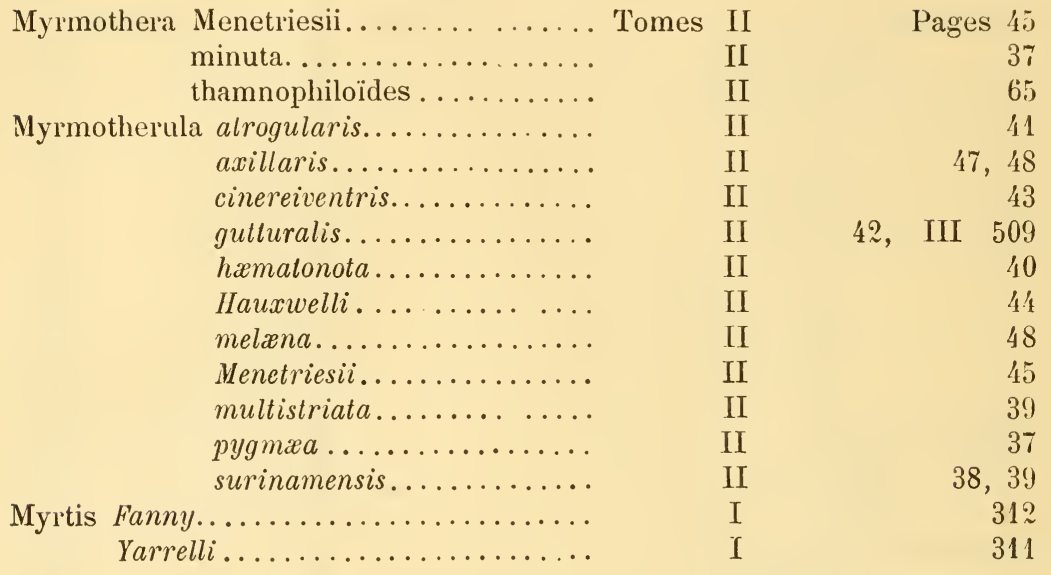

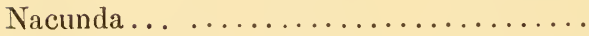

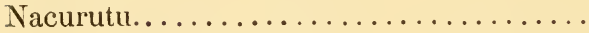

chorreado................

189

192

Nænia inca.......................

446

Nasica albicollis ................. II

171

longirostris .................. II

171

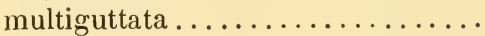

180

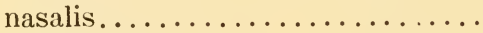

171

triangularis

177

137

Nauclerus forcipatus. ............ I

137

furcatus.............. I

442

Nectarinia luteola................ I

435

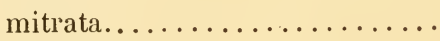

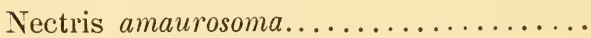

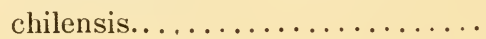

III

III

463

463

Negriblanco .....................

Neinei

II

294

Nemosia auricollis

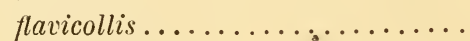

II

511

fulvescens...............

508

guira................. II

guirina.............. II

510,554

gularis $\ldots \ldots \ldots \ldots \ldots \ldots \ldots \ldots \ldots \ldots \ldots$ III

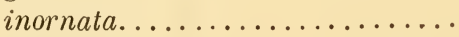

nigricollis ..............

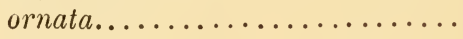

pectoralis 
Nemosià pileata................. Tomes II

sordida .............. II

Pages 513

torquata................ I

508

432

Neocheridon tibialis...............

242

Neomorphus Pucherani.............

183

Neopipo cinnamomea...............

346

rubicunda............... II

346

Neops spirurus............... II

167

Neorhynchus nasesus.............. III

Nephocates fumigatus .............

Nertus rufifrons.................

Nisus concentricus.................

leucorrhous.................

magnirostris...............

- pileatus...................

striatus ................

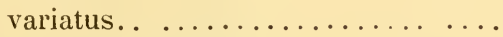

Noctua dominicensis..............

ferruginea $\ldots \ldots \ldots \ldots \ldots \ldots \ldots$

ferox...................

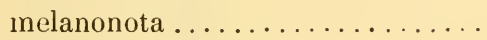

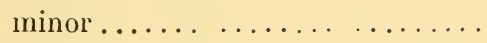

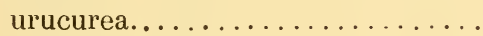

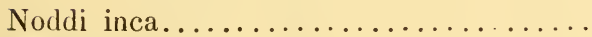

Nonnula brunnea.................

frontalis................

ruficapilla.................

Notherodius guarauna...............

Nothocercus atricapillus.............

Nothocrax urumutum ...............

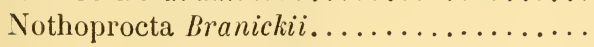

curvirostris...............

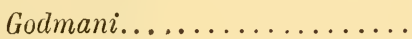

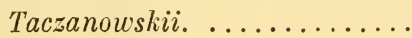

Nothriscus picatus................

Numenius borealis................ III

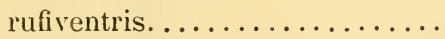

III

Nyctiardea pileata..................

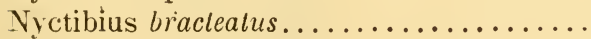
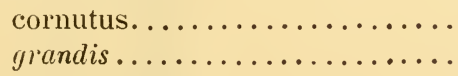

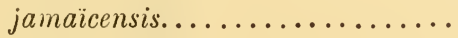

longicaudatus. 
Nyctibius urutau................. Tomes I

Nycticorax americanus.............. III

Pages 207

III 407

Gardeni.............. III 407

nævia............... III

407

obscurus............... III

406

pileatus.............. III

408

violaceus............... III

405

Nyctidromus albicollis.

226

Nyctiphrynus ocellatus.

215

Nyphocates fumigatus.

ㄱ?

0

Oceanites oceanica................ III

463

Ochthites brevicauda................

293

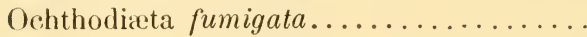

188

fuscorufus...............

signala..............

190

189

Uchthoeca fumicolor...............

191

fumigata ..............

188

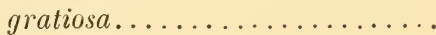

199

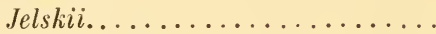

198

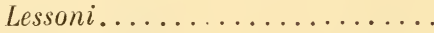

195

leucometopa...............

193

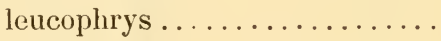

193

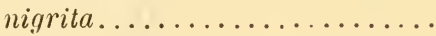

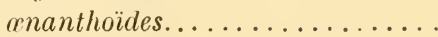

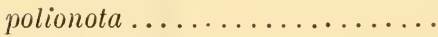

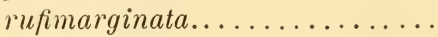

rufipectoralis. . ............

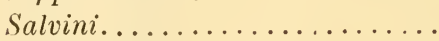

stictoptera..............

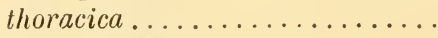

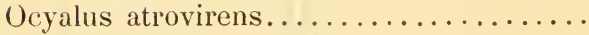

cristatus . . . . . . . . . . . .

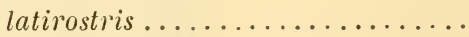

panayensis ..............

viridis.

197

192

193

196

194

200

201

197

106

404

402

402

405

Ocypetes torquatus............... III

283

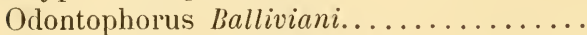

pachyrhynchus...........

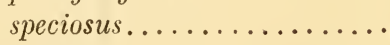

290

III

287

III

stellatus................

III

288

III

289

OEdicnemus superciliaris............

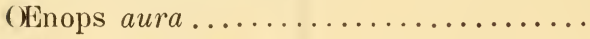

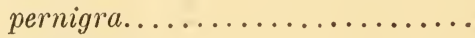

urubitinga 
Onchorhynchus Castelnaudi Tomes II

Onocrotalus fuscus............... III

Pages 295

Hernandezi ........... III

425

thagus .............. III

425

424

Opisthocomus cristatus............ III

$26: 2$

hoazin............. III

262

Opetiorhynchus ferrugineus..........

nigrofumosus. .........

104

110

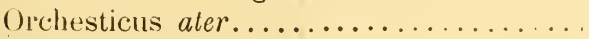

II

II

547

()rchilus ecaudalus...................

pileatus. . . . . . . II

230

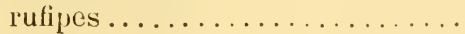

233

Oreonympha nobilis.

344

Oreophilus ruficollis................ III

347

Oreotrochilus Estella.

277

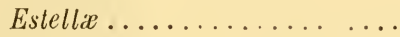

277

leucopleurus..............

278

melanogaster.

279

Oriolus americanus ...............

427

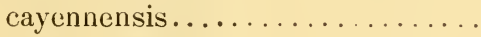

417

chrysocephalus.............

416

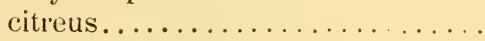

404

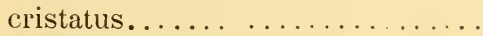

404,405

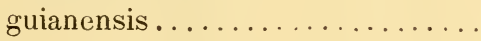

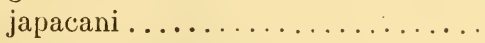

icterocephalus...............

leucopterus ................

melaleucus...............

mexicanus . . . . . . . . . . . . .

musicus...............

oryzivorus . ..............

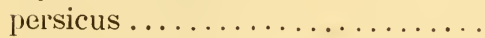

rufirostris. ..............

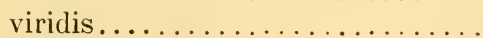

Ornismya Allardi ..................

amazili .................

amethystina..............

amethystoïdes..............

Audeberti..................

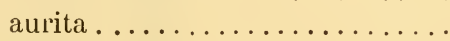

bicolor . ...............

chrysura................

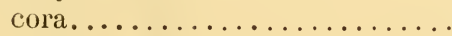

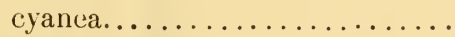

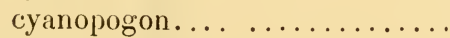

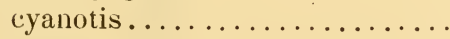

Delattrii ................

delphinie

427

502

425

504

504

426

417

435

411

405

105

358

406

317

317

410

363

411

336

314

411

306

369

299 


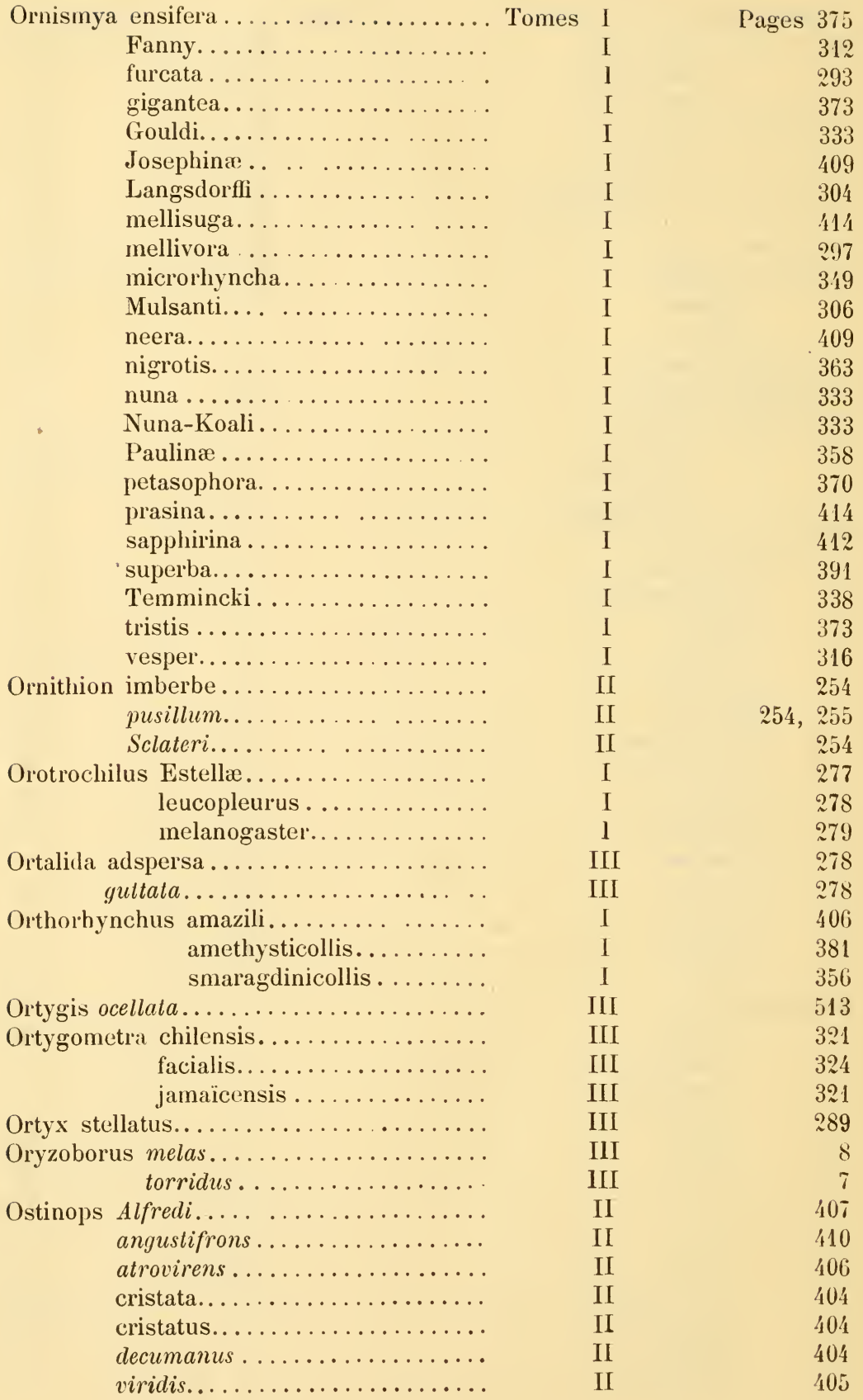


Ostinops Yuracarium............. Tomes II

Ostralega atra............... III

Pages 403

palliata.............. III

351

351

Otus americanus.

brachyotus................. I

194

mexicanus............... I

galopagoensis.............. I

192

194

Ourax galeata $\ldots \ldots \ldots \ldots \ldots \ldots \ldots \ldots$ III

266

mitu ... $\ldots \ldots \ldots \ldots \ldots \ldots$ III

pauxi ................ III

266

266

Oxyurus dorsomaculatus

116

Pachynus brachyurus.............. III

216

Pachyrhamphus albogriseus...........

II

361

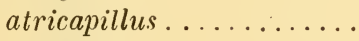

II

cinereus ..............

II

$360,36 う$

leucogaster...........

II

360

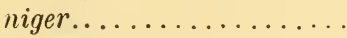

360

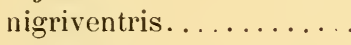

II

363

pectoralis.............

363

rufescens.............

II

358

spodiurus. . . . . . . . . . .

368

squamatus............

362

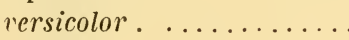

366

viridis...............

366

Pachyrhynchus albifrons ...........

melanocephalus.........

rufescens.............

semifasciatus ..........

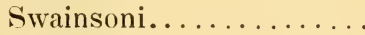

II

II

II

II

359

II

365

354

368

353

365

Paille en queue de Cayenne............

436

127

127

fluviatilis..............

fulvus.................

halirtus.

104

197

295

I'anoplites Mathewsi..............

P'aroaria gularis

Parula brasiliana................

$$
\text { pitiay } u m i \ldots \ldots \ldots \ldots \ldots \ldots \ldots
$$

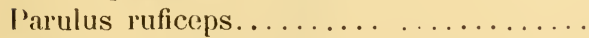

Parus auricapillus ................

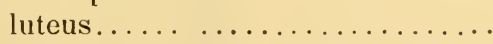

Parra chilensis $\ldots \ldots \ldots \ldots \ldots \ldots \ldots$ 
Parra variabilis................ Tomes III viridis................ III

Pages 331

III 331

Passerina guttata.............. III

oryzivora ...............

421

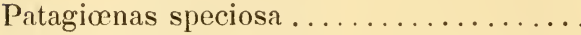

II

Patagona gigas.................... 373

Pato roxo y negro ................ 470

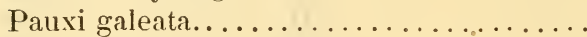

$$
\text { mitu. }
$$

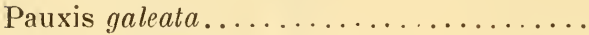

Pelecanoïdes Garnoti.................

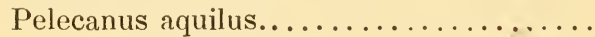

carolinensis ..............

425

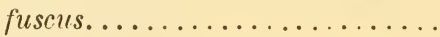

Gaimardi.................

leucocephalus ..............

Molinx..................

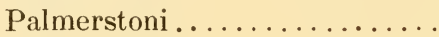

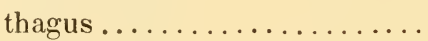

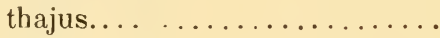

424

vigua ..................

424

429

360

Pelidna Schinzi................. III

Pendulinus cayanensis .............. II

Penelope aburri ................. III

albipennis............... III

boliviana..................

III

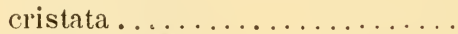

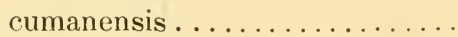

III

III

268

?.67

276

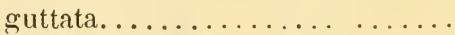

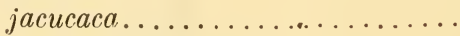

III

267

leucolophus ................

III

III

276

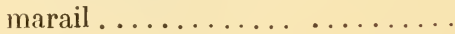

III

268

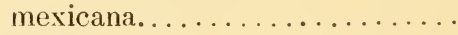

III

470

pipile.................

976

rufiventris................

III

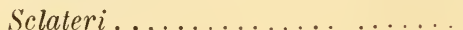

III

III

Percnostola fortis..................

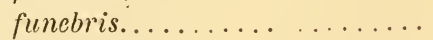

Peristera chrysauchenia .............

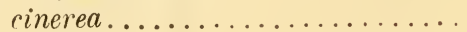

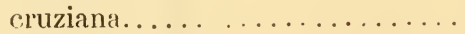

frenata. . . . . . . . . . . . . . .

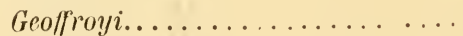

jamaïcensis..............

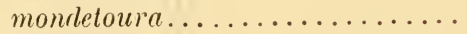

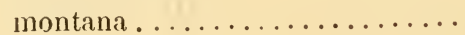


Petasophora anaïs................ Tomes I

Cabanidis................ I

Pages 367

cyanotis............... I

369

cyanotus ............. I

369

Delphinæ............. I

369

Geoffroyi.............. I

Gouldi.............. I

jolata $\ldots \ldots \ldots \ldots \ldots \ldots \ldots \ldots \ldots \ldots$ I

365

370

serrirostris

367

370

Petrochelidon albilinea............. III

503

albiventris. . ......... I

239

cinerea.............. I

243

leucoptera........... III

503

littorea............. III

503

ruficollaris..............

III

503

tibialis

242

Pezites brevirostris

428

loyca $\ldots \ldots \ldots \ldots \ldots \ldots \ldots$ II

428

Pezus niambu

III

997

zabele.

III

302

Phacellodomus frontalis.

II

143

striaticeps . . . . . . . . .

II

144

Phæolæma xquatorialis...............

292

Phä̈thon xthereus.

III

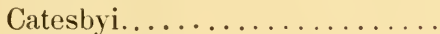

III

436

flavirostris...............

III

436

melanorhynchus ...........

III

136

436

Phaëthornis amaura............... I

272

268

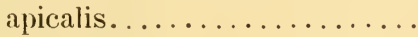

Bourcieri.................

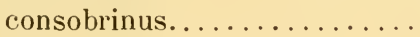

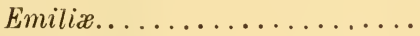

griseigularis..............

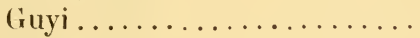

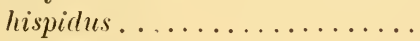

malaris...............

Moorei .................

niqricinctus .............

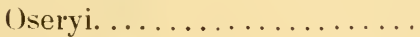

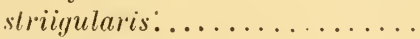

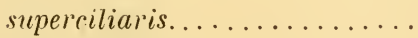

s!jrinatophorus.............

villosus. . . . . . . . . . . . . .

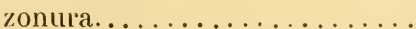

Phac̈tusa magnirostris. 
Phalacrocorax brasiliensis........... Tomes III

cirriger............. III

Pages 4:9

Gaimardi............. III

431

niger.................

III

431

Phalaropus angustirostris............

III

429

australis ...............

382

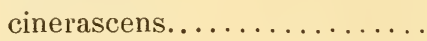

III

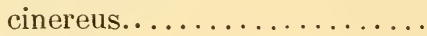

III

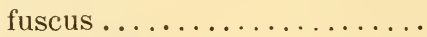

III

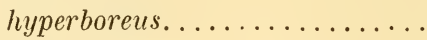

III

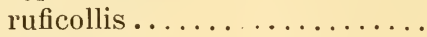

III

vulgaris................

III

382

Williamsi...............

Phalcobænus montanus ..............

III

III

382

382

382

381

382

382

382

Pharomacrus antisianus.............

III

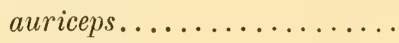

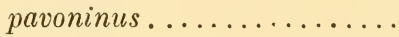

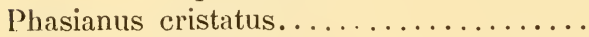

hoazin................

III

III

III

262

III

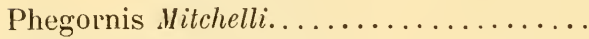

262

III

Pheucticus aureiventris..............

III

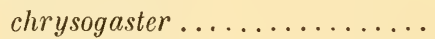

chrysopeplus ..............

III

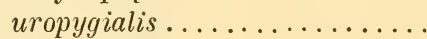

III

III

Philydor erythrocercus...............

erythropterus.............

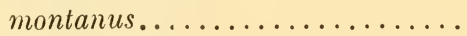

ochralæmus...............

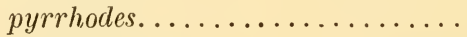

ruficaudatus..............

striaticollis...............

subflavescens.............

subfulvus ...............

turdinus.

II

372

Phlœocryptes melanops..............

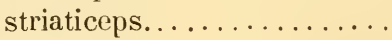

Phlogopsis erythroptera............

Phønicocercus coccineus . ..........

Phœnicoparrus andinus...............

Phøenicopterus andinus..............

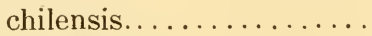

ignipallialus ............

Phœnicosoma Azaræ................

III

Phoenicothraupis Carmioli.............

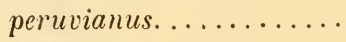

rubica.............. 
Phœnisoma ardens. Tomes II

Pages 497

Azara............... . II

495

bivittata. ............. II

497

Pholeoptynx cunicularia............. I

174

hypogæa ............ I

Phonasca melanura.............. II

174

$\begin{aligned} \text { Phonasca } & \text { melanura } \ldots \ldots \ldots \ldots \ldots \ldots\end{aligned}$

447

saturata. ............ II

$44 \stackrel{2}{\circ}$

xanthogastra

443

II

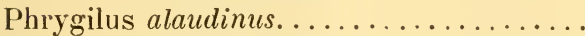

Aldunati..................

III

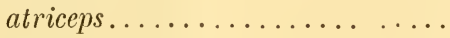

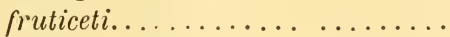

III

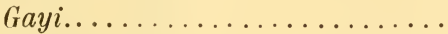

III

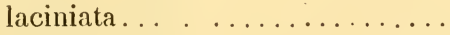

III

plebejus................

III

ocularis.

III

rusticus.

III

39

rusticus . . . ......................

III

39,40

unicolor

III

Phyllomanes flavoviridis..............

Phyllomyias cinereocapilla...........

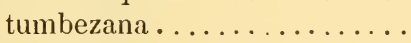

II

38

38

445

251

II

252

Phytotoma Bloxhami.

II

Molinæ. ...............

II

394

Raimondii...............

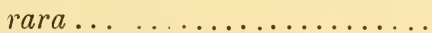

II

394

395

silens..................

Pjaya brachyptera ...............

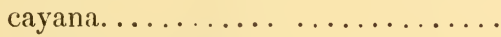

cayana nigricrissa............

III

erythrophthalma.............

III

Mehleri....................

III

melanocorypha ..............

III

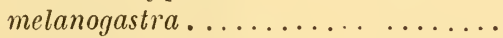

III

minuta................ III

nigricrissa............. III

rutila................. III

186

186

189

186

189

188

187

186

187

Pica chloronotus

Picolaptes cinnamomeus........... II

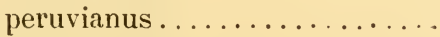

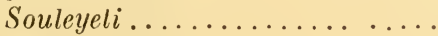

I'icumnus albosquamatus. ...........

III

Castelnau 


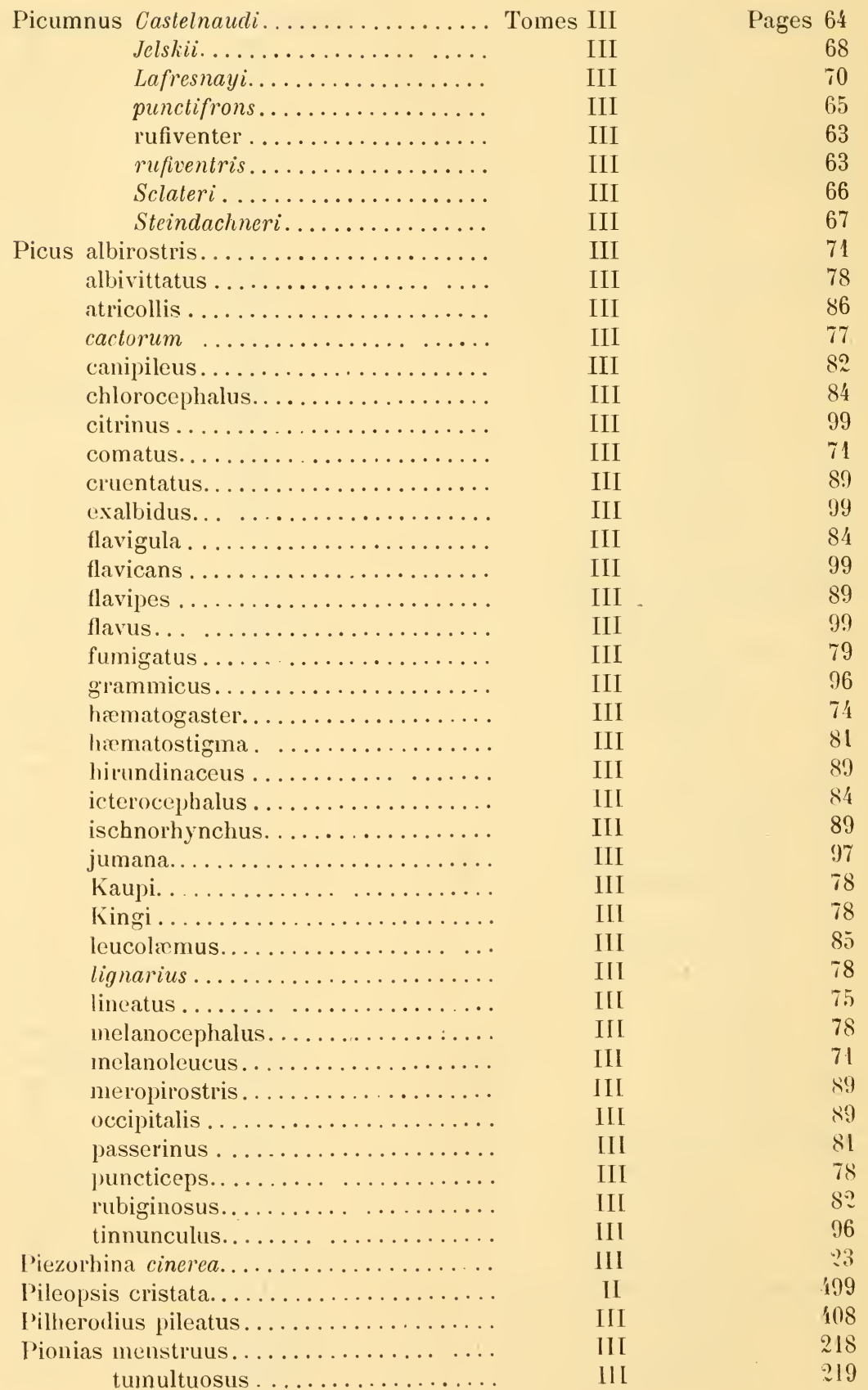


Pionus Barrabandi

Tomes III

Pages 229

menstruus.

III

218

tumultuosus

III

219

Pipile cumanensis.

III

276

pipile.

III

276

Pipilo mystacalis............... II

533

Pipilopsis flavigularis..............

fulviceps..............

superciliaris.

II

514

532

517

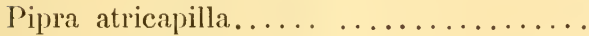

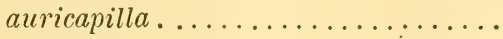

chloris

II

334

chloromeros.................

II

339

cinerea....................

II

360

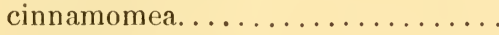

II

346

coccinea ...................

II

374

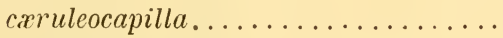

II

344

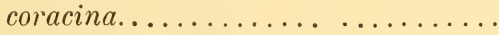

II

342

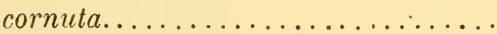

II

337

coronata.

II

343

cristata.

II

340

cyaneocapilla.

343

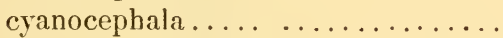

II

439

elata

256

erythrocephala..............

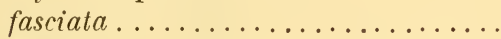

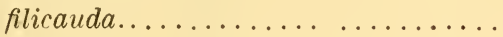

filifera ...................

Isidori .

II

II

II

II

338,340

II

337

336

II

336

312

leucocapilla.

341

leucocephala.

207

leucocilla

341

manacus

349

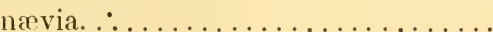

nigricollis................

peruviana

374

373

purpurea..................

pyrocephala ... . . . . . . . .

340

346

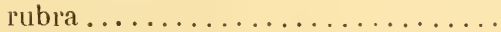

340

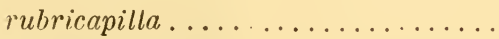

338

rupicola $[v.] \beta \ldots \ldots \ldots \ldots \ldots \ldots$

strigillata

373

striolata.

torquata.

virescens.

II

3.15

Pipræides castaneoventris.

II

451

Pipreola aureipectus 
Pipreola Lubomirskii................ Tomes II

melanolæma ............... II

Pages 377

Riefferi............... II

376

viridis ............... II

375

viridis intermedia............

Pipridea albiventris ...............

castaneiventris ................ II

451

melanonota .............. II

450

melanonota venezuelensi........ II

150

venezuelensis............. II

450

Piprites chlorion.................

Tschudii................

334

334

Pitangus sulphuratus...............

286

Pithys albifrons..................

albifrons peruviana............

leucaspis...................

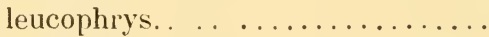

lunulata..................

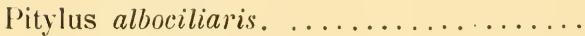

aureiventris . ............

chrysogastel ..............

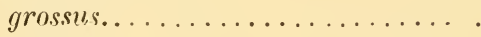

olivaceus . .............. .

III

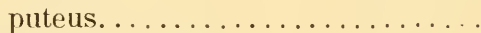

Placellodomus frontalis.............

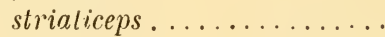

Platalea ajaja...................

coccinea................

412

incarnata. ..............

rosea ....................

412

Platyrhynchus albigularis...........

225

bicolor...............

chrysoceps ............

coronatus .............

Duponti. . . . . . . . . .

nuchalis.............

305

307

359

380

paganus............. .

$: 62$

platurus. . . . . . . . . .

ruficauda. . . . . . . . . . .

$\operatorname{senex} . \ldots \ldots \ldots \ldots \ldots . . . .$.

sulphurescens $\ldots \ldots \ldots \ldots$.

Platyurus niger.

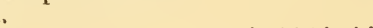

Plotus anhinga.

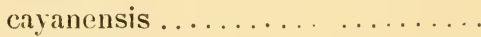

melanogaster.............. 
Pluvialis dominicensis.

'Tomes III

torquata.............. III

virginiana $\ldots \ldots \ldots \ldots \ldots \ldots$ III

Podager nacunda.................

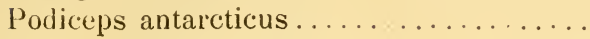

bicornis................

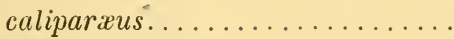

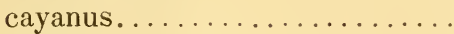

chilensis ..............

dominicanus.

dominicus.

leucotis

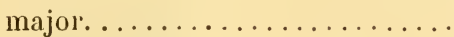

micropterus ..............

occipitalis ..............

Rollandi..................

Podilymbus antarcticus..............

Podoa surinamensis................

Pœecilonetta bahamensis..............

Pœcilothraupis ignicrissa............

igniventris............

lacrymosa............

palpebrosa.............

Pœocephalus melanocephalus

xanthomerus.............

Pogonotriccus ophthalmicus............

Polemistria Verreauxii...............

Polioptila albiloris. ...............

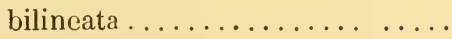

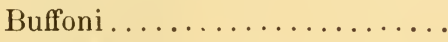

nigriceps..............

parvirostris...............

Polyborus brasiliensis..............

caracara.................

chimachima ............

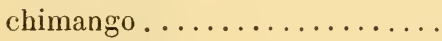

megalopterus............

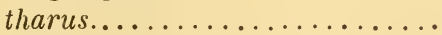

vulgaris...............

Polydacnis angelica..............

Polythmus hypoleucus..............

leucoproctus.............

leucorrhous................

Polyxemus bombus................

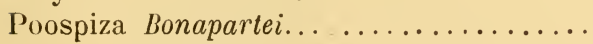

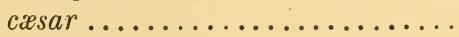

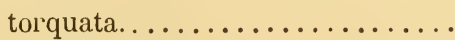

Popelairia tricholopha.
III

III

III

III

III

III

III

III

III

III

III

III

III

III

III

III

II

II

II

II

III

III

II

I

I

III

III

I

I
Pages 3 4:

342

342

208

498

492

493

492

493

495

495

494

492

497

493

494

498

490

483

482

482

480,481

480

227

226

250

300

452, III 504

505

453, III 505

453

505

92

92 
Porphyrio cinereus............... Tomes III

Porphyriops melanops.............. III

leucopterus............ III

Pages 32:

326

326

Porzana carolina................. III

320

cayanensis............... III

323

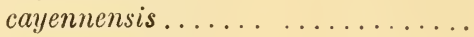

III

323

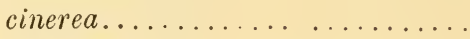

III

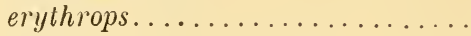

III

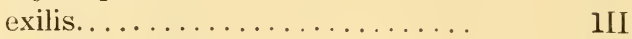

322

facialis.................

III

325

fasciata . . . . . . . . . . . .

III

Hauxwelli................ III

322

324

jamaïcensis... ...........

III

324

viridis ...................

III

324

321

323

Prasites phœe pyga................

Premnocopus multiguttatus. ..........

Presbys peruanus.................

Prionites æquatorialis ..............

dombeyanus...............

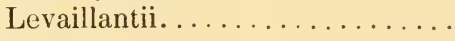

Martii................. III

microstephanus. . .........

$\operatorname{momota} . \ldots \ldots \ldots \ldots \ldots \ldots$

III

platyrhynchus

III

rubricapillus.

III

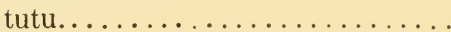

Prionirhynchus platyrhynchus.........

III

Procellaria brasiliana...............

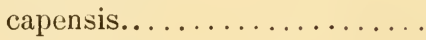

III

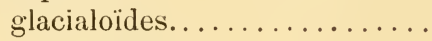

nævia..................

III

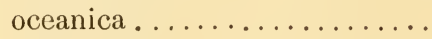

Wilsoni.................

III

Procnias occidentalis ...............

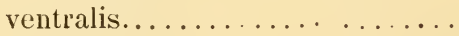

II

viridis................

Procnopis argentea ...............

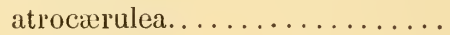

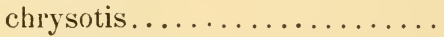

nigricincta . . . . . . . . . .

Parzudakii ..............

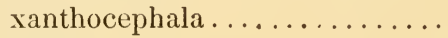

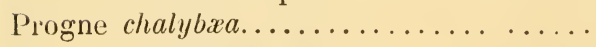

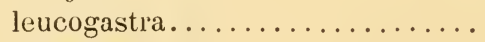

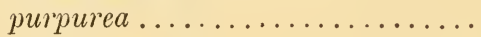

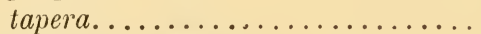

Prymnacantha Langsdorffi. 
Psaris atricapillus... . . . . . . .... Tomes II

aurantius..... $\ldots \ldots \ldots \ldots \ldots \ldots$ II

cayanus................. II

Cuvieri.................. II

II

guyanensis .

niger.................. II

roseicollis............... II

semifasciatus ............ II

versicolor................ II

Pages 360

360

354

viridis................ . II

359

$35 \dot{4}$

363

358

353

366

II

359

Psarocolius caudacutus.............. II

421

chrysopterus........... II

croconotus............. II

417,424

gymnops................

II

420

jamacai...............

$4 ? 6$

icteronotus. ............

420

II

411

mesomelas............ II

nigerrimus............

415

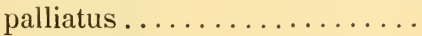

Pseudocolaptes auritus..............

Boissonneaui...........

435

II

145

semicinnamomeus.......

145

145

Pseudomitris columbiana ...........

II

II

II

III

Psittaca guianensis.................

III

Psittacara chloroptera...............

III

199

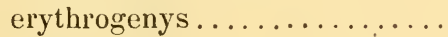

III

198

melanura.................

III

202

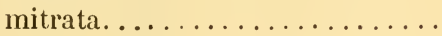

III

196

rubrilarrata .............

III

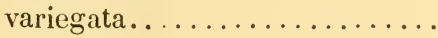

III

198

202

Psittacula andicola.................

III

211

brasiliensis icterocephalus.....

III

209

brasiliensis uropygio cyaneo...

III

21 ?

calestis....................

III

214

crassirostris..............

III

215

griseifrons . ..............

III

205

melanorhyncha............

III

213

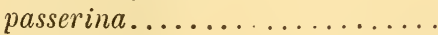

III

Sclateri..................

III

212

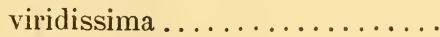

III

III

III

213

Psittaculus Sancti-Thomæ............

Psittacus æstivus.................

III

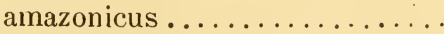

amazonicus brasiliensis .......

III

amazonicus pœeilorhynchus....

ambiguus............... .

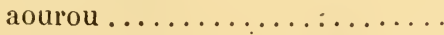

III 


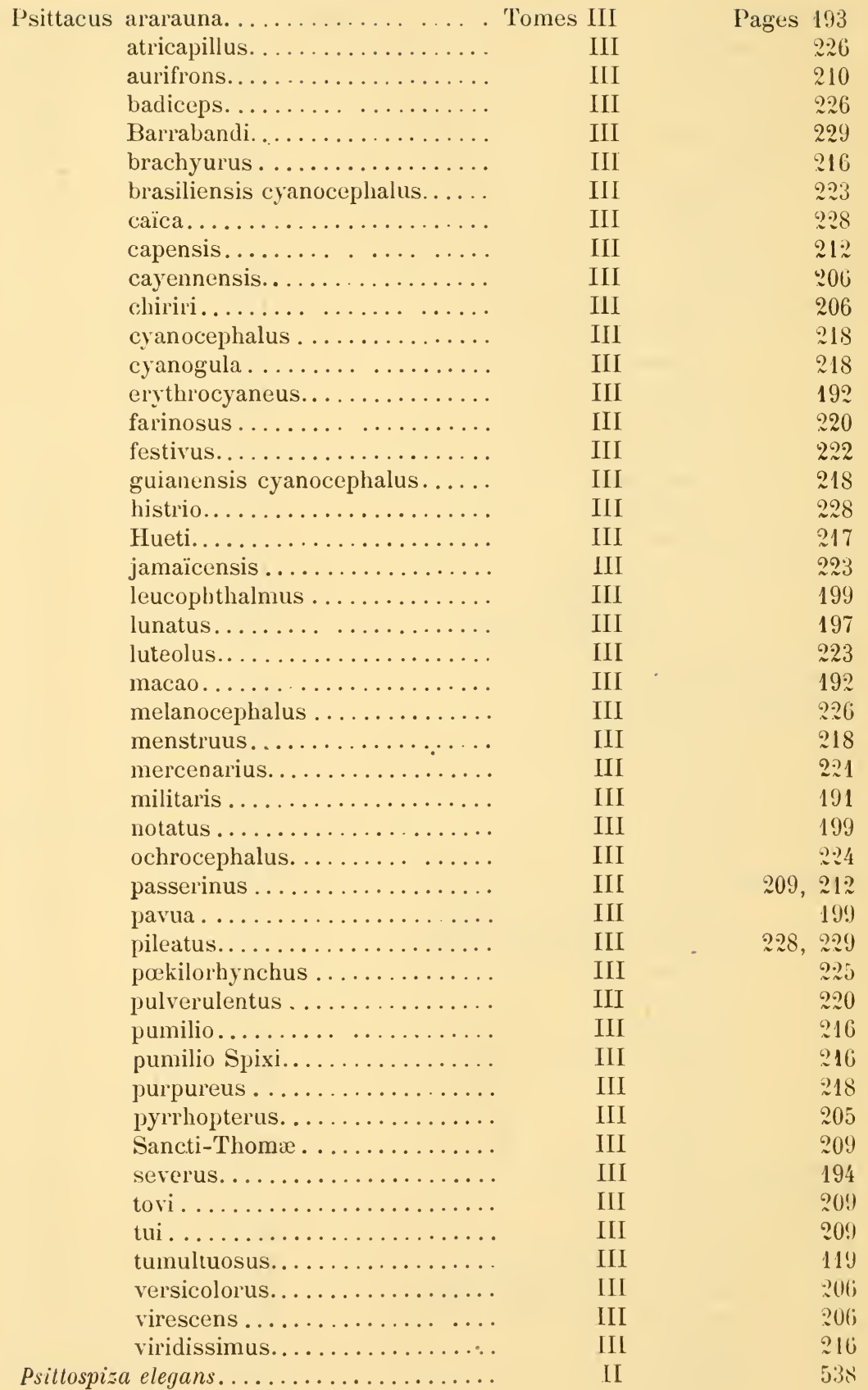


Psittovius jugularis............... Tomes III

Psophia crepitans............... III

leucoptera............... III

Pages ?08

385

Pteroglossus $A z a r x . . \ldots \ldots \ldots \ldots \ldots \ldots$ III

385

atrogularis........... III

149

Beauharnaisii.......... III

159

castanotis............ III

152

çeruleicinctus ...........

151

derbianus...............

III

160

flavirostris............. .

III

158

Gouldi................

III

148

hrematopygus...........

III

156

Ilumboldti...............

III

161

hypoglaucus ...........

III

149

Langsdorffi. .............

III

157

lepidocephalus

154

Lichtensteini.............

III

152

pluricinclus..............

III

160

Pœppigi..............

III

151

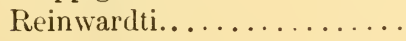

152

ulocomus.

153

III

$15 ?$

Pterophanes Temmincki..............

Pteroptochus acutirostris............

femoralis..............

532

magellanicus ............

Ptilogonys griseiventer.............

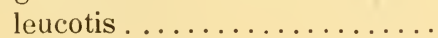

Ptyonura albifrons............... II

inentalis .................. II

218

rufivertex .............. II

216

Puffinaria Garnoti..................

III

465

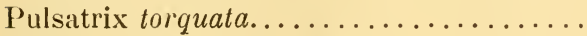

Pygmornis amaura..............

griseogularis.............

nigricincta............

nigricinctus. ...........

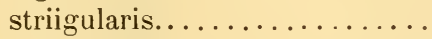

Pygoptila maculipennis ..............

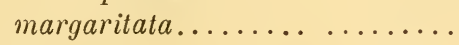

272

270

272

272

272

II

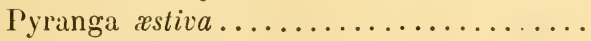

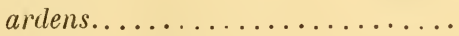

$A \approx \operatorname{arx} \ldots \ldots \ldots \ldots \ldots \ldots \ldots$

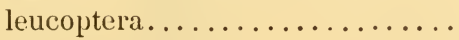

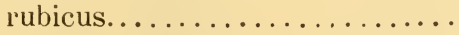

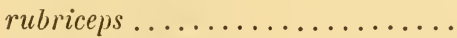

II 
Pyriglena funebris.............. Tomes II

Pages 57

picea.................... II

tyrannina............ II

56

$\operatorname{serva.\ldots \ldots \ldots \ldots \ldots \ldots \ldots \text {II}}$

54

II 56

Pyrocephalus obscurus... ..........

II

311

rubineus.............. II

rubineus coronatus........ II

307,310

rubineus obscurus......... II

310

311

Pyroderos granadensis.

392

Pyrrhocoma fulviceps..............

Pyrrhodiglossa brunneiventri..........

532

420

Pyrrhomyias cinnamomeus..........

300

Pyrrhophæna leucophæə.............

407

Pyrrhorhynchus arcuatus........... II

379

Riefferi............ II

375

Pyrrhula carulea brasiliana........... III

leucomelas............... III

telasco................ III

Pyrrhulopsis Hueti... ............

III

Luciani. ............. III

203

Pyrrhura melanura...............

III

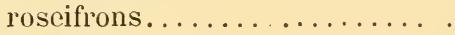

III

202

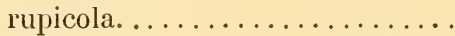

204

Souancei

III

201

III

201

\section{Q}

Querquedula angustirostris...........

cæruleata..............

cyanoptera. ............

475

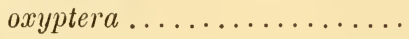

III

476

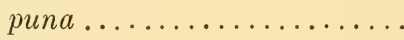

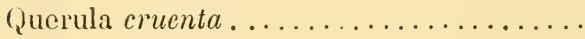

$\operatorname{minor} \ldots \ldots \ldots \ldots \ldots \ldots \ldots$

rubricollis................

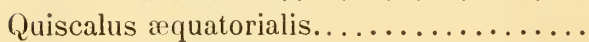

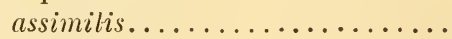

peruvianus...............

III

478

389

II

358

II

II

433

II

II

\section{$\mathbf{R}$}

Rallus ardeoïdes..................

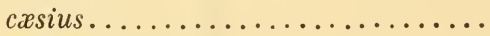

carolinus.................

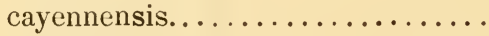

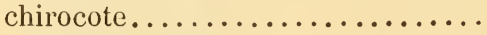

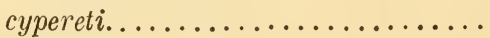


Rallus gigas................. Tomes III

jamaïcensis............. III

Pages 387

kiolo

III

321

limicola . . . . . . . . . . . . . . III

323

maximus.............. III

314

melanops. . $\ldots \ldots \ldots \ldots \ldots \ldots \ldots \ldots$ III

318

nigricans.............. III

326

peruvianus.

317

plumbeus.

III

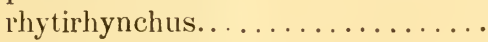

III

313

III

319

Salinasi .................. .

316

semiplumbers $\ldots \ldots \ldots \ldots \ldots$

III

321

stolidus

III

314

virginianus

III

320

viridis.

III

314

III

323

Ramphotrigon ruficauda.............

II

284

Recurvirostra andina..............

III

38 '

himantopus .......... III

383

Regerrhinus cayennensis.............

megarhynchus...........

Regulus Azaræe.

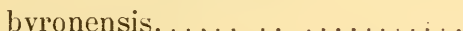

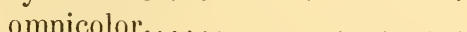

plumulosus ...............

Rhamphastos ambiguus . . . . . . . . .

Azaræ................

culminatus...............

Cuvieri................

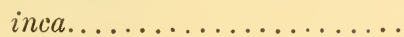

$\operatorname{momota} . . . \ldots \ldots \ldots \ldots$.

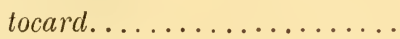

Rhamphocænus albiventris..........

melanurus........... .

144

141

243

II

243

243

239

II

145

III

149

III

146

III

147

III

146

III

108

III

145

II

II

Rhamphocelus aterrimus............

II

493

atrosericeus............

II

493

ignescens.............

II

491

jacapa..............

Luciani................

II

491

nigrogularis...........

Rhamphodon anaïs.

II

494

II

491

367

microrliynchum ........

olivaceus ..............

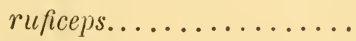

Stanleyi.............

Vulcani ...................

Rhamphopis atrococcineus............

atrosericeus............. 
Rhamphopis jacapa............. Tomes II

Luciani.............. II

nigrogularis........... II

Pages 491

493

491

Rhea americana................. III

500

Rhinoptynx mexicanus.............

Rhodopis vesper................

Rhopias Hauxwelli..................

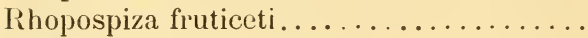

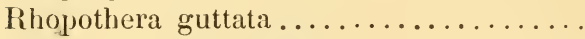

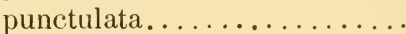

Rhyacophilus solitarius............

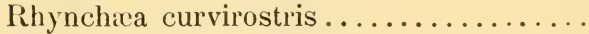

hilairea..................

Hilarii............... . III

semicollaris...............

III

378

Rhynchaspis maculatus............ III

480

mexicana............. III

480

Rhynchocyclus fulvipectus...........

megacephalus .........

II

283

peruvianus..............

poliocephalus..........

II

281

ruficauda.............

sulphurescens. .........

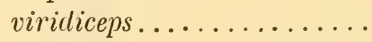

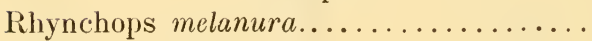

III

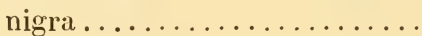

III

Rhynchotis perdix..............

Rollandia leucotis.................

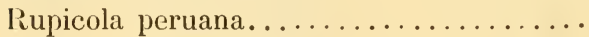

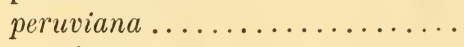

III

282

437

437

306

III

II

494

\section{S}

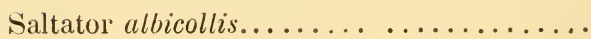

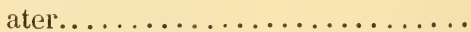

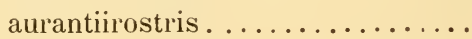

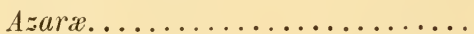

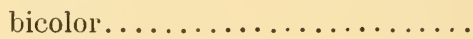

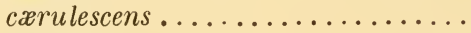

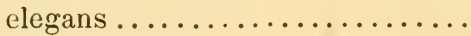

flavidicollis............... II

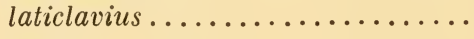


Saltator olivaceus.

Tomes II

Pages 540

olivascens.............. II

Riefferi............... II

543

similis.

538

superciliaris............. II

5 il

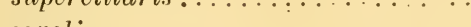

Sappho caroli

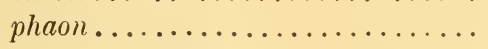

Sarcorhamphus condor..............

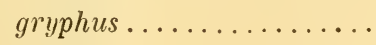

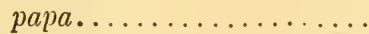

Sarkidiornis jubata.

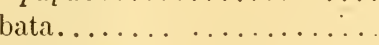

Sarochalinus rufogularis.. . . . . . . . .

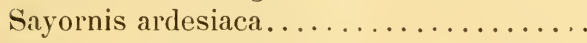

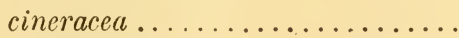

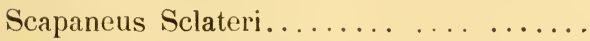

II

541,544

I

337

336

75

75

75

III

468

508

317

204

Scaphidura barita................

crassirostris . .........................

435

435

Scaphidurus ater...............

435

Scaphorhynchus audax............

chrysocephalus ........

sulphuratus.

288, 289

293

294

Schiffornis major................

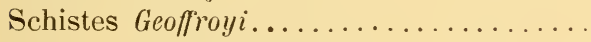

Schistochlamys speculigera............

Schizoca palpebralis...............

Sclerurus caudacutus................

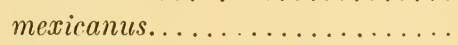

olivascens...............

Scolopar alba................

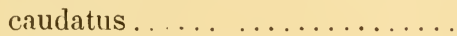

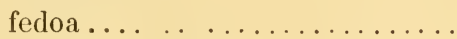

flavipes . ...............

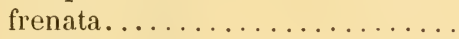

grisea...................

leucophæa.................

marmorata ..............

melanoleuca .

novaboracensis.

35 ?

365

546

130

114

115

II

115

III

415

417

III

379

III

367

375

373

373

379

III

365

III

III

Paykulli............... . . III

373

semipalmatus.............

III

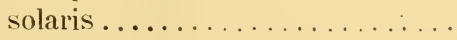

III

vociferus...............

III

Scops brasilianus

brasiliensis...............

choliba..................

cristatus ................

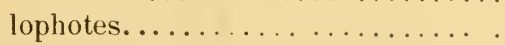

portoricensis 
Scops usta

Tomes

Pages 187

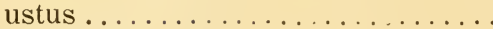

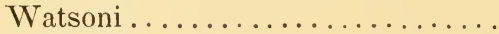

187

185

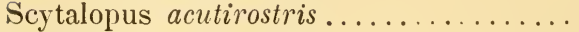

532

femoralis...............

532

magellanicus...............

529

sylvestris............ I

531

Selenidera Gouldi...................

III

156

Langsdorffi.

154

Reinwardti.

III

III

Sericophila anthracina.

II

153,155

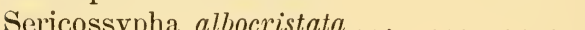

II

208

Serphophaga cinerea...............

hypoleuca.............

pœecilocerca.............

ruficeps................

Serpophaga cinerea...............

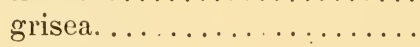

hypoleuca .............

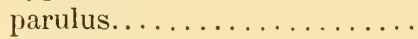

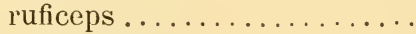

Serrirostrum sittoïdes. ..............

Setophaga Bairdi.................

budytoïdes.............

chrysogastra .............

coronata................

melanocephala.............

nigricincta..............

ruticilla.................

verticalis................

Sirystes albogriseus . .............

Sitta surinamensis................

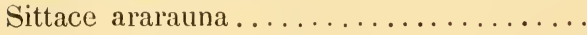

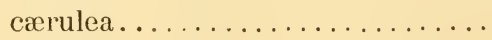

coccinea .................

Illigeri. . . . . . . . . . . . . .

maracana ...............

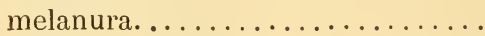

militaris.................

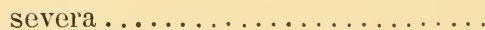

Sittasomus amazonus...............

olivaceus..............

perlatus................

stictolæmus ..............

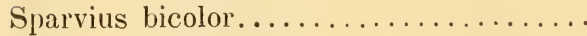

cærulescens............ .

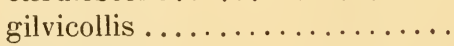

platypterus...............

II

387

236

238

203

237

จ36

236

238

239

237

417

480

238

473

476

479

468

507

481

?87

III

III

192

III

195

III

III

III 
Sparvius semitorquatus ........... Tomes I

Spathura cissiura.............. I

Pages 160

peruana.............. I

329

rufocaligata . . . . . . . . . I

327

solsticialis............. I

326

324

Spatula platalea................. III

480

Speotyto cunicularia............... I

174

Spermophila castaneiventris ... ....... III

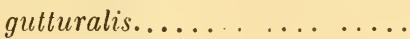

III

13

luctuosa................

III

masesus............... III

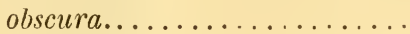

III

ocellata.................

III

17,18

olivaceo-flava.............

III

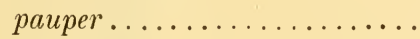

III

rufirostris ............

telasco.................

III

simplex.

III

Spheniscus Humboldti.

III

Sphenura frontalis

III

499

Spiza jacarina.

Spizætus ater

fuscescens. . . . . . . . . . . . . . .

Mauduyti.................

melanoleucus ..............

niger..................

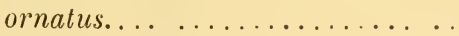

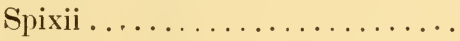

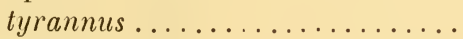

variegatus

Sporophila castaneiventris ...........

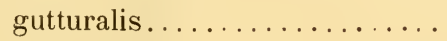

III

luctuosa ............. III

rufirostris. .............

III

telasco $\ldots \ldots \ldots \ldots \ldots \ldots \ldots \ldots$ III

Squatarola australis

III

cinerea. ..............

III

helvetica ..............

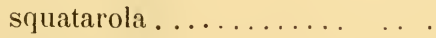

III

Steatornis caripensis..............

caripensis peruviana.........

Steganura addl ..................

cissiura ...............

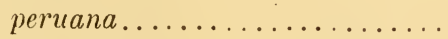

solstitialis. 
Steganurus peruanus............... Tomes I

Stelgidopteryx ruficollis............. I

Pages 327

uropygialis ............ I

246

247

Stenopsis xquicaudata...............

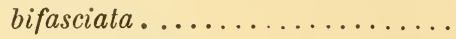

222

maculicaudus..............

221

216

nigrescens............. I

218

Stercorarius chilensis............. III

458

pomatorhinus........... III

Sterna acutirostris............. III

458

445

arctica $\ldots \ldots \ldots \ldots \ldots \ldots \ldots$ III

441

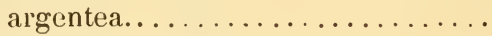

III

445

Bergii. ....................

III

439

Cassini.................. III

440

cayana................ III

439

cayennensis............... III

439

comata............... III

442

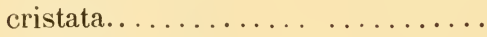

III

439

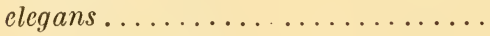

442

erythrorhynchus.............

III

439

exilis................. III

445

Frobeenii................ III

443

galericulata............... III

hirundinacea............... III

439,442

hirundo ................. III

440

inca................... III

440,441

lorata $\ldots \ldots \ldots \ldots \ldots \ldots \ldots \ldots \ldots \ldots$ III

446

445

macrura............... III

441

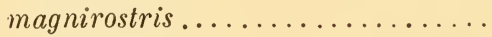

III

$\operatorname{maxima} \ldots \ldots \ldots \ldots \ldots \ldots \ldots$ III

.438

meridionalis ..............

439

III

440

paradisea............... III

441

Pikei.................. ' III

portlandica $\ldots \ldots \ldots \ldots \ldots \ldots$ III

441

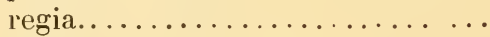

III

441

439

superciliaris...............

III

444

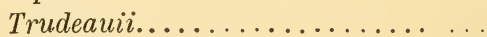

III

Sternula superciliaris... .......... .

III

445

II

Stigmatura budytö̈des..............

III

238

Strepsilas collaris.................

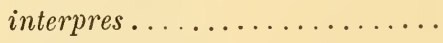

melanocephalus............

III

Strix accipitrina................

ægolius ... $\ldots \ldots \ldots \ldots \ldots \ldots$

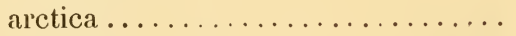

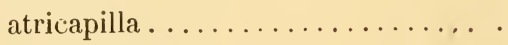

brachyotus ................

brachyura ............... 


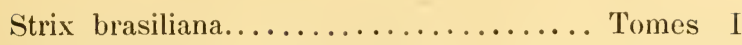

Pages 185

caspia.................. I

194

choliba................... I

185

clamata.................. I

192

cristata.................. I

188

crucigera................. I

185

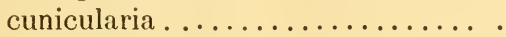

171

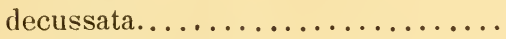

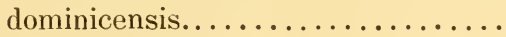

185

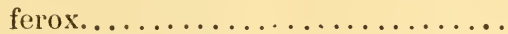

174

ferruginea.................

178

178

flammea americana. ............

flammea Guatemalæ.............

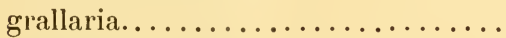

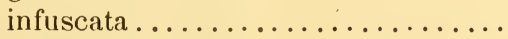

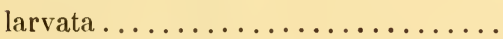

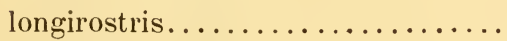

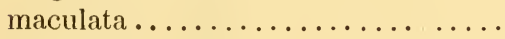

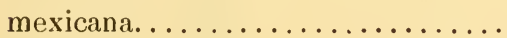

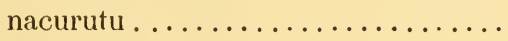

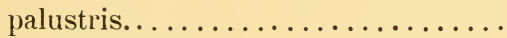

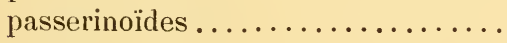

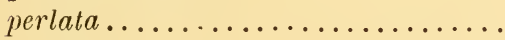

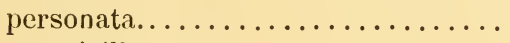

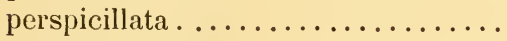

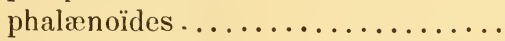

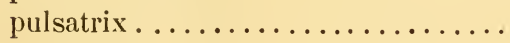

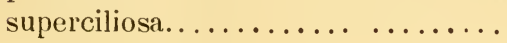

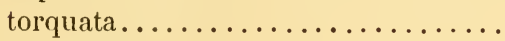

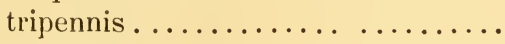

ulula....................

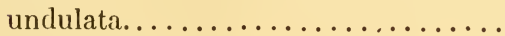

Sturnella bellicosa ..................

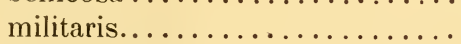

Sturnus holosericeus. ..............

loyca $\ldots \ldots \ldots \ldots \ldots \ldots \ldots \ldots \ldots$

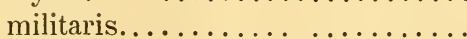

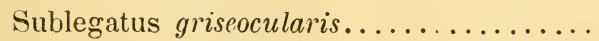

Suiriri cabeza blanca................

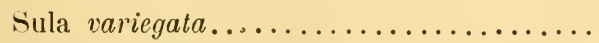

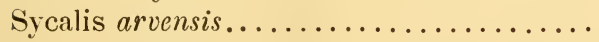

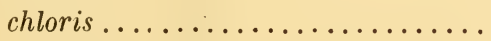

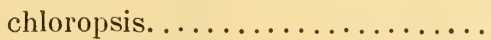

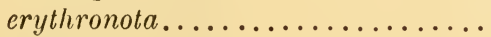

flave $l a \ldots \ldots \ldots \ldots \ldots \ldots \ldots \ldots$

lutea.......................

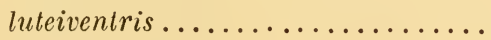

Raimondii. 
Sycalis uropygialis............... Tomes III

Sylbeocyclus chilensis............. III

dominicus............ III

Pages 58

493

496

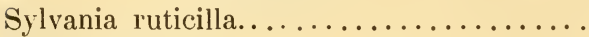

507

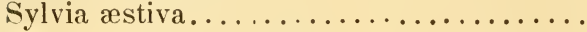

III

506

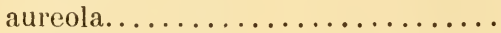

III

467

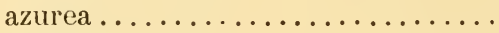

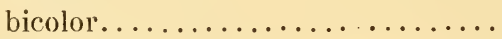

bifasciata................ I

465

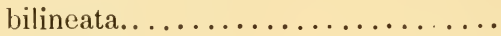

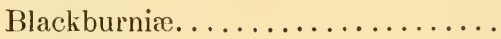

III

505

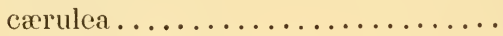

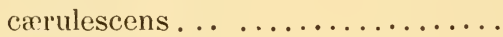

I

465

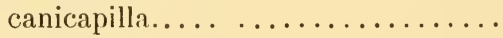

433

469

carolinensis $\ldots \ldots \ldots \ldots \ldots \ldots \ldots$ III

506

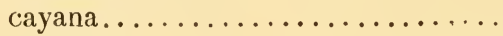

Childreni.................

506

citrinea ..................

506

506

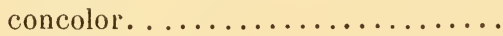

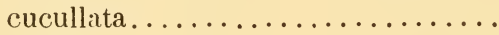

flava.................. III

506

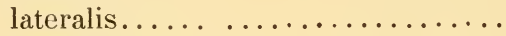

magellanica .............

464

529

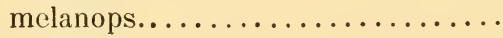

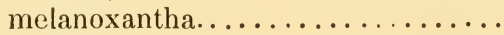

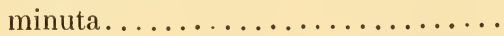

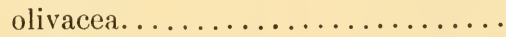

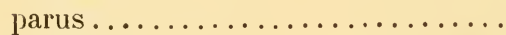

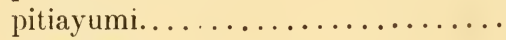

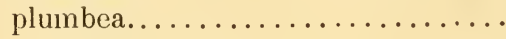

populorum ................

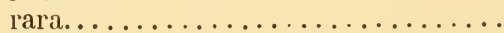

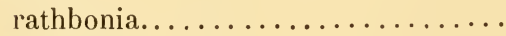

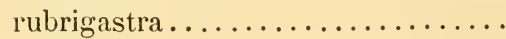

II

511

463

443

464

463

trochilus ..................

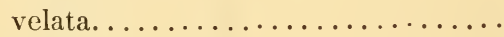

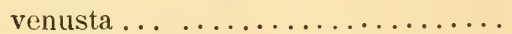

Sylvicola aureola. . ...............

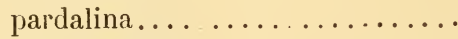

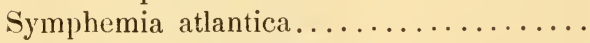

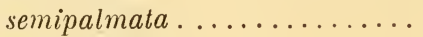

Synallaxis angithaloïdes.............

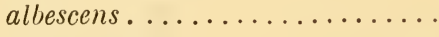

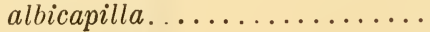

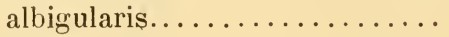

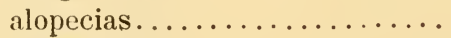

antisiensis. 
Synallaxis Arequipæ.............. . Tomes II

Azaræ ............... II

brunneicauda............. II

brunneicaudalis........... II

cinnamomea............ II

cisandina.............. II

curtata................. II

dorsomaculatus........... II

elegans............... II

elegantior............... II

flammulata............. II

frontalis............... II

fruticicola................ II

furcata................ II

graminicola.............. II

humilis................. II

Pages 137

122

124

121

127

133

135

116

$12: 2$

122

139

122,123

123

134

140

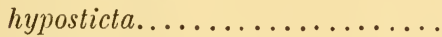

138

136

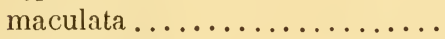

II

126

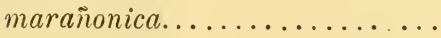

II

130

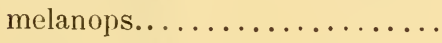

116

multostriata .............

139

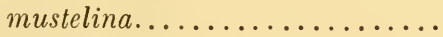

127

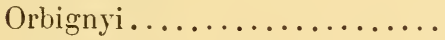

137

palpebralis.................

130

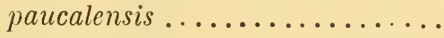

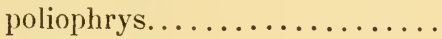

propinqua.................

131

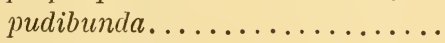

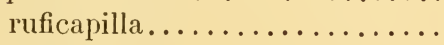

rufifrons ................

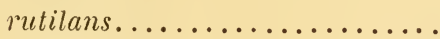

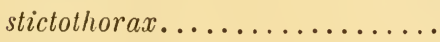

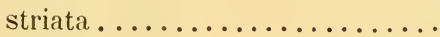

terricolor................

thelotis.................

tithys ..................

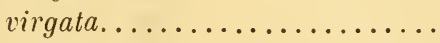

vulpecula...............

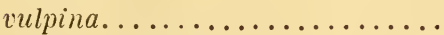

Syndactyla rufosuperciliata ...........

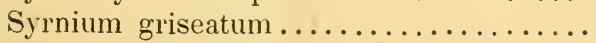

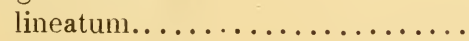

melanonotum ..............

perspicillatum . ............

squamulatum .............

virgatum ..............

zonocercum .

II

II

II

II

II

122

II

126

II

142

II

122,124

II

143

II

132

II

126

II

121

126

120

129

141

128

128

157

188

182

184

180

182

182

$18 \%$ 
Tachornis andicola................ Tomes I

Tachuris rubrigastra............... II

Pages 232

Tachybaptus dominicanus............. III

243

dominicus................. III

496

dominicus............... II

495

Tachycineta albilinea.................

503

Tachypetes aquila..................

Tachyphonus albitempora..............

III

427

II

513

cirrhomelas..............

502

cristatellus ..............

II

502

cristatus................

II

502

gularis ................

II

flavinucha.............

III

484

fringilloïdes .............

II

lacrymosus. .............

481

leucopterus...............

melanoleucus..............

napensis ................

nigerrimus ..............

penicillatus...............

phoniceus...............

III

505

II

504

503

504

499

505

quadricolor...............

506

rubescens................

rufiventris...............

saucias..................

serrirostris ..............

Suchi.................

sumptuosus ..............

surinamus...............

surinamus napensis........

versicolor................

Tænioptera erythropygia...............

Talpacotia cinnamomea...............

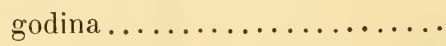

Tamatia melanotis..................

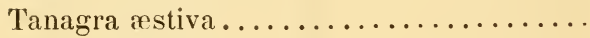

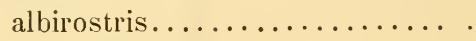

albocristata.................

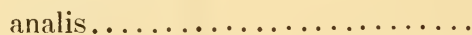

(Arremon) assimilis............

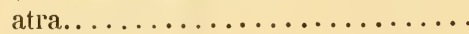

atrosericea .................

aureata ....................

auricapilla..................

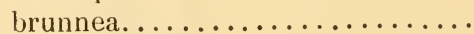

brunneinucha...............

III

503

505

504

506

$48 \mathrm{i}$

503

503

500

187

246

246

129

494

491

387

479

531

547

493

439

506

502

529 
Tanagra cana................. Tomes II

chilensis ............. II

Pages 486

chrysogastra............ II

coccinea.............. II

457

439, 447

cœlicolor. .............. II

494

485

458

cristata ............... II

502

cristatella ................

III

cucullata................

28

II

483

cyanicollis................

472

cyanocephala...............

II

490

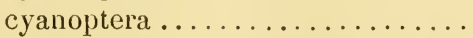

II

486

Darwini..................

II

I [

488

decumana...............

II

544

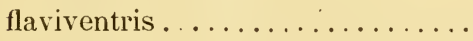

II

464

frugilegus................

II

fusca $\ldots \ldots \ldots \ldots \ldots \ldots \ldots$

III

488

gularis.

III

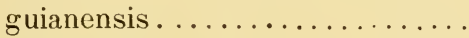

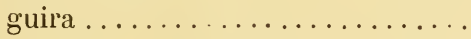

448

510

gyrola ...................

I

463

icterocephala . . . . . . . . . . . .

II

508

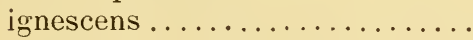

II

491

igniventris . . . . . . . . . . . . . .

II

482

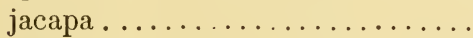

II

II

491

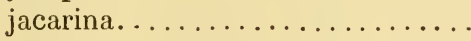

III

Luciani .................

II

$\operatorname{magna} . . \ldots \ldots \ldots \ldots \ldots \ldots$

II

494

Maximiliani . . . . . . . . . . . .

II

539

490

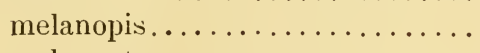

melanoptera...............

nigerrima ...............

nigricollis...............

nigrigula...... . . . . . . .

nigrogularis ..............

olivacea..................

olivascens.................

olivina...................

palmarum melanoptera.........

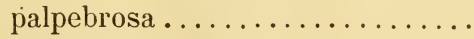

Parzudakii.................

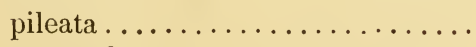

pompadora...............

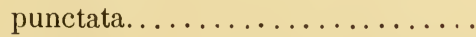

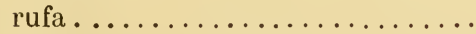

II

547

II

487

II

504

II

II

439,510

II

II

II

II

II

II

II

II

II

II

442, 494

486

547

486

480

174

513

II

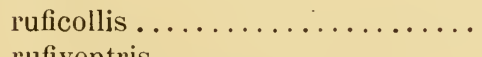

III

rufiventris............... II

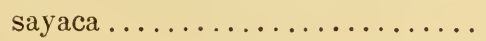

II 
Tanagra Schranki................. Tomes II

speculigera.............. II

Pages 459

striata................. II

511

supcrciliaris.............. II

489

variegata............... II

541,544

yeni ................... II

Tanagrella callophrys............... III

494

457

elegantissima........... II

510

iridina ............... II

Tantalus albicollis................ III

453

453

417

albus.................. III

brevirostris.............. III

415

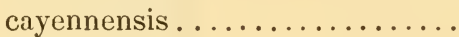

414

III

coco .................. III

420

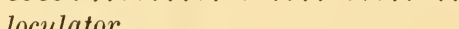

415

III

melanopis .............. III

414

plumicollis................

III

Tanypeplus pavoninus.

III

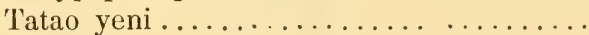

II

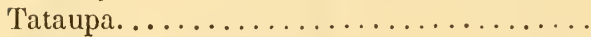

Terenura callinota .................

III

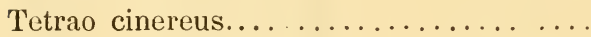

Thalasseus elegans.................

magnirostris............

Thalassidroma Wilsoni.

II

III

III

III

III

Thalassøeca glacialö̈des...............

III

417

414

176

457

297

Thalassoïca glacialoïdes..............

III

464

464

polaris ............ . III

464

tenuirostris............

Thalurania Jelskii.................

III

nigrofasciata...........

Tschudii................

viridipectus.

Thamnophilus albicans.............

albinuchalis. ...........

amazonicus.............

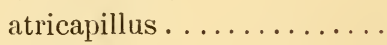

axillaris ..............

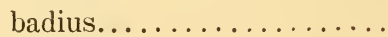

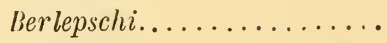

cæsius ...............

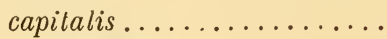

caudacutus............

corvinus...............

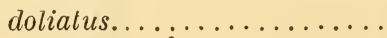

fasciatus . .............

fuliginosus . ..........

griseus. 
Thamnophilus guianensis........... Tomes I

Pages 448

guttatus............ II

59

hyperythrus .......... II

24

leucauchen........... II

leuconotus.............. II

lineatus............. II

1,17

loretoyacensis........... II

luctuosus............. II

major.............. II

melanchrous........... II

melanocephalus......... II

melanoceps..............

II

melanurus. ............ II

mentalis.............. II

murinus...............

myotherinus ...........

II

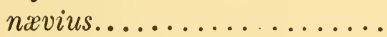

II

12

nævius albiventris.........

II

65,67

olivaceus.............

II

8,9

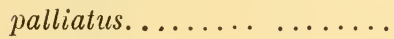

plumbeus..............

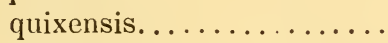

radialus ...............

rubiginosus............

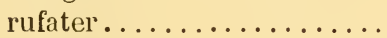

rufifrons..............

rufiventris ............

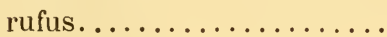

schistaceus ............

subundinus.............

subandinus major.........

subfasciatus............

tenuifasciatus ............

tenuipunctatus ...........

transandeanus...........

Tschudii.............

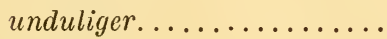

Thamnistes rufescens...............

Thamnomanes glaucus .... ..........

Thaumastura cora.................

Thaumatias Bartletti

fluviatilis $\ldots \ldots \ldots \ldots \ldots \ldots \ldots \ldots$

Taczanowskii.............

Thaumatoëssa mirabilis.............. 
Thinocorus orbignyanus ............ Tomes III rumicivorus............. III

Swainsonii ............ III

Pages 281

283

Thrasaëtos harpyia...............

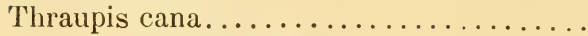

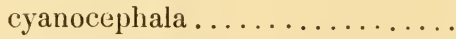

II

486

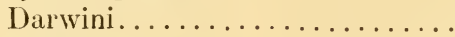

II

490

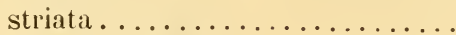

Threnetes cervinicauda..............

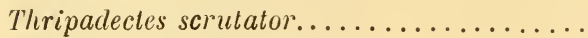

Thriprobrotus Warszewiczi...........

Tripophaga aurita................

Thryophilus fulvus..................

leucotis..................

superciliaris ..............

Thryothorus albiventris............

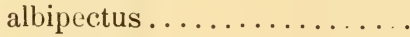

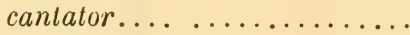

coraya

griseipectus .............................

leucotis ..............

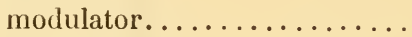

prostheleucus . . .........

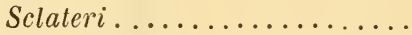

superciliaris ............

Thylopsis fulvescens..............

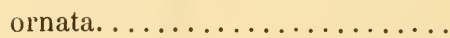

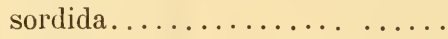

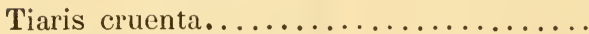

II

II

488

489

260

I

146

II

173

II

145

I

517

516

514

518

514,516

519

517

517

516

508

513

520

514

508

507

508

II

III

Tigrisoma brasiliense..............

III

401

Cabanisi................ III

404

Salmoni............... III

402

Tinactor fuscus.

II

114

Tinamotis Pentlandi...............

III

310

Tinamus atricapillus ...............

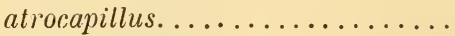

III

294

cæerulescens ...............

III

cinereus.................

III

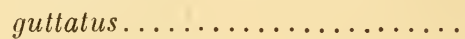

III

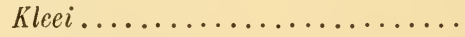

III

major.................

noctivagus ...............

III

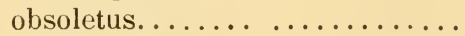

ruficeps..................

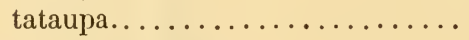

Tinnunculus cinnamomeus............

cinnamominus...........

sparverius..............

III

302

296

292

III 
Tityra albitorques............... Tomes II

cayana................ II

cinerea................

nigra.................. . II

354

semifasciala............... II

II

viridis............... II

'Todirostrum cinereum............. II

chrysocotaphum .......... II

Pages 355

ecaudatum ............ II

maculatum ............ II

pulchellum ............ II

Sclateri.............. II

signatum............ II

spiciferum........... II

squamæcristata......... II

Todus cinereus................. II

II

leucocephalus..............

II

marginatus. ................

II

melanocephalus ...............

poliocephalus.... ..........

II

II

Totanus bartramius ... . . . . . . . . . $\quad$ III

354

363

353

359

225,226

227

234

228

227

226

228

229

230

$2: 5$

207

365

295

285

III

371

caligatus ................

368

campestris................

III

371

chloropygius..............

III

368

crassirostris...............

III

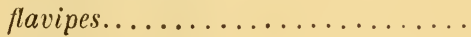

III

364

fuscocapillus . .............

III

367

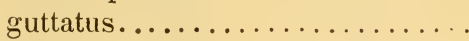

III

367

longicauda ...............

III

368

leucopyga................

III

371

macropterus. ..............

III

367

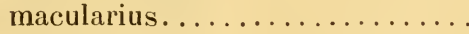

III

368

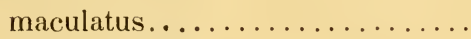

III

369

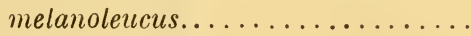

III

melanopygius... ......... III

natator............... III

punctatus.............. III

366

365

371

367

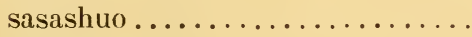

III

368

semipalmatus.

III

solitarius................ III

speculigerus............. III

variegatus...............

Triccus cinereus

III

II

365

364

368

364

Trichas nigricristatus

Trichoglossus aurifrons

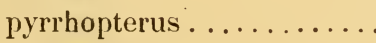

III

Trichothraupis quadricolor. 


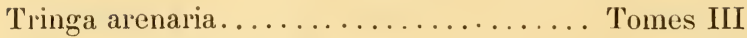

atricapilla ............... III

Auduboni ................ III

australis ............... III

Pages 353

378

363

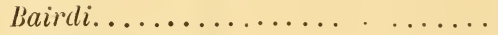

355

bartramia ...............

III

359

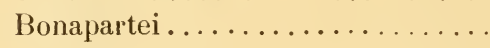

III

371

borealis .

III

360

brevirostris. ...............

III

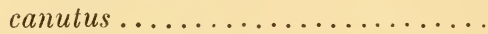

III

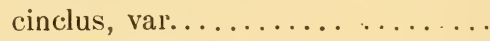

III

348

362

354

cinerea.................

III

360

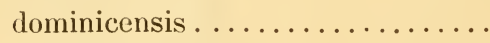

III

354

Douglasi................

III

ferruginea................

III

356

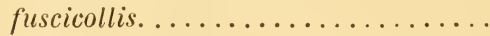

III

grisea..................

III

helvetica ................

III

himantopus................

III

hyperborea...............

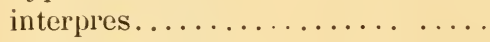

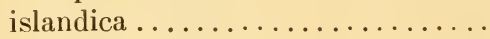

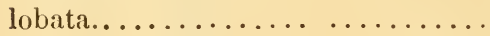

III

III

III

III

macularia ................

III

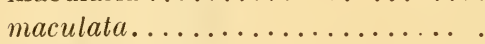

III

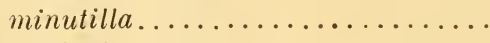

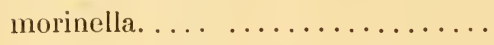

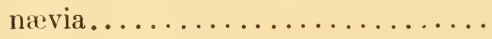

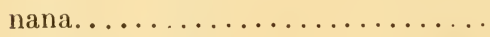

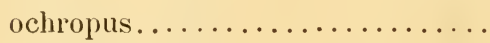

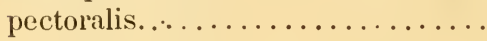

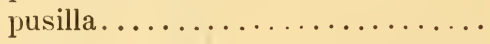

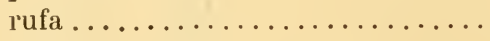

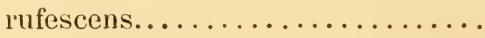

semicollaris ...............

semipalmata...............

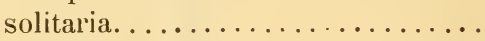

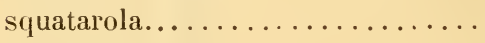

subruficollis. ...............

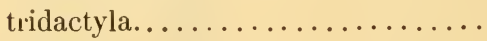

III

III

III

III

III

III

III

III

III

III

363

354

356,360

338,354

338

363

382

349

354

382

369

356

358

349

354

358

368

356

358,362

III

355

370

378

III

362

368

338

III

370

353

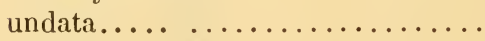

III

355

uniformis ...................

III

355

varia.....................

III

virgata......................

Wilsoni. .

III

III

Tringites rufescens

III

Tringoïdes macularius

Trochilus addx.

III 
Trochilus æneicauda ............... Tomes albus................. I amazilia .............. I amethysticollis.......... I amethystinus. .......... [ anaïs............... I angustipennis.......... I apicalis............... I Aspaziæ.............. I atricapillus.............. I atrimentalis.............. I Audeberti.... . . ......... I aurescens............. I auritus $\ldots \ldots \ldots \ldots \ldots \ldots \ldots \ldots$ I Bourcieri.............. I brachyrhynchus ............ I brevicauda............. I brasiliensis ............. I Crciliæ............... I

campestris... ..........

caroli ...................

Castelnaudi...............

chionogaster..............

chrysogaster...............

chrysurus................

cacruleus ................

Condamini..............

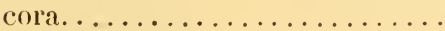

cupreicauda................

cupripennis...............

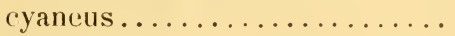

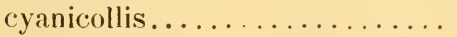

cyanopterus ..............

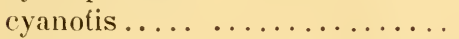

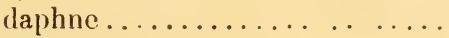

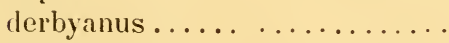

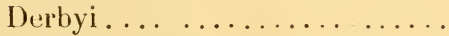

Eimiliae .................

Estella..... ......... I I

fasciatus............ I

ferrugineus $\ldots \ldots \ldots \ldots \ldots \ldots \ldots$ I I $\quad$ I

fimbriatus............. I $\quad 997$

fulvifrons............. I 112

furcatus ............. I

Gayi.............. I

Geoffroyi............. I

gigas.............. I 373 
Trochilus Guimeti ................ Tomes I

hirsutus ............... I

hispidus............... I

hypoleucus.............. I

inornatus................ I

insectivorus............. I

Johannæ................ I

Labrador ................. I

(Lampornis) leucogaster....... I I

Langsdorffi . ............ I

latirostris............... I

lazulus ................. I

(Lesbia) gracilis............ I

(Lesbia) smaragdinus......... I

leucocrotaphus ............. I

longirostris ...............

mango.....................

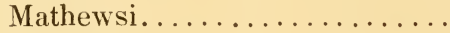

mazeppa...................

melanogenys...............

mellivorus...............

mirabilis..................

mocoa...................

nigricollis................

nigrofasciatus.............

(Ocreatus) rufocaligatus.......

opacus ..................

Oseryi.....................

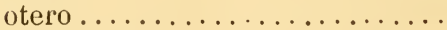

petasophorus..............

phæopygus................

platurus ....................

Popelairi...................

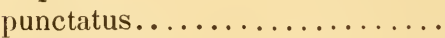

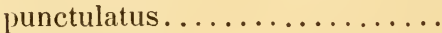

quadricolor . ................

ruficeps ...................

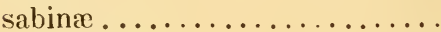

sapphirinus ................

Saulæ....................

Schreibersi ..............

serrirostris................

Stanleyi ...................

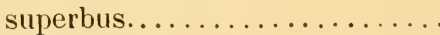

superciliosus...............

thalassinus...............

tyrianthinus..............

Turnieri..................

Pages 364

261

265

400

359

289

285

312

400

304

412

281

330

334

363

391

281

295

261

361

297

318

334

281

293

326

353

266

287

370

415

327

302

281

281

281

348

361

412

283

289 
Trochilus Verreauxi.

Tomes I violicauda.............. I

violifrons . .............. I

Troglodytes arada .................

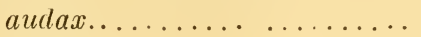

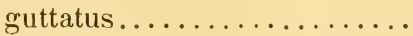

leucophrys .............

murinus...............

solstitialis ................

tecellata..................

tessellatus ................

Trogon antisianus

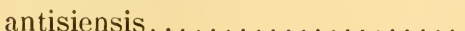

atricollis.

auratus.

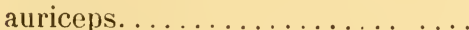

caligatus...............

castaneus .................

chrysochlorus..............

collaris....................

curucui.

heliothrix..... ....................

lepturus.................

leverianus................

melanopterus .............

melanurus...................

meridionalis .. . ...........

nigricaudatus . . . . . . . . . .

pavoninus ...............

personalus heliothrix.

personatus propinquus.

pulchellus ................

purpuratus................

rainonianus.................

rosalba..... . ...........

rufus...................

strigillatus. . ... ........

tenellus .................

variegatus . . .............

violaceus. ...............

viridis.

Trupialis bellicosa.

loyca

militaris

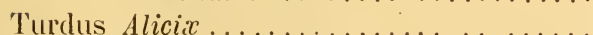

atricapillus. .............

aquaticus
Pages 300

281

285

508

525

512

512

522

521

522

522,524

177

177

172

163

175

173

163

172

163

III

III

III

III

III

III

III

III

III

III

III

III

III

III

III

III

III

III

III

III

III

III

III

II

II

II
$163,168,172$

166, 167

172

170

170

168

171

169

176

$16 \%$

166

166

177

165

171

163

172

168, 170

172

165

$1 \% 0$

170, 172 
Turdus atrosericeus.............. Tomes I

brasiliensis ............. I

Pages 496

brunneus .............. I

502

chiguanco .............. I

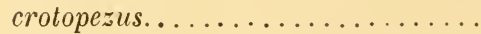

494

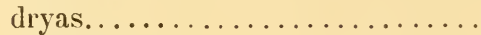

492

fumigatus ...............

484

fuscater $\ldots \ldots \ldots \ldots \ldots \ldots \ldots \ldots$

493

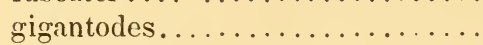

494

Hauxwelli. ...................

(Hylocichla) Swainsoni, $\beta$ Aliciæ. ignobilis .................

leucomelas. ..............

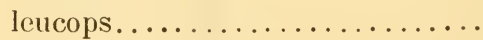

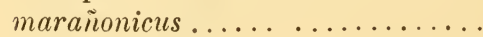

nigriceps. ................

phxopygioüdes...............

phropygus ..............

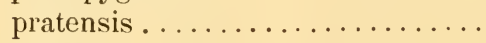

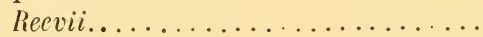

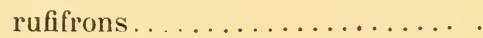

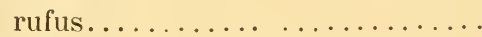

serranus..................

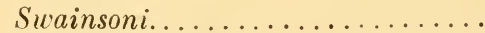

Swainsoni, var. Alicix.........

thilius....................

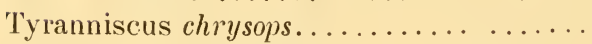

cinereiceps.............

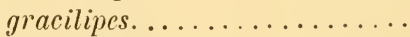

nigricapillus.............

viridiflavus .............

viridissimus. .............

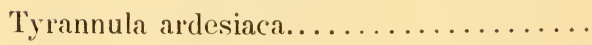

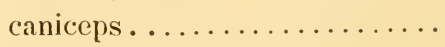

cineracea...............

ferruginea.............

fumigata.............

megacephala .............

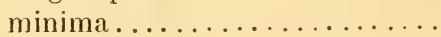

phœnicura... ............

superciliosa . . ..........

Tschudii ................

Tyrannulus albocristatus.............

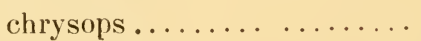

cinereiceps .............

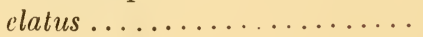

Tyrannulus nigricapillus ... .........

rufipectus...., .......... 
Tyrannus albicollis ............. Tomes II

Pages 273

atrifrons............. II

291

audax................ II

288, 289

aurantio-atro-cristatus ........

331

auriflamma .............. II

331

Boissonneauti........... II

188

borealis .............. II

316

calcaratus............. II

$3 \% 0$

carnivorus ............. II

294

cayennensis............ II

275

cinchoneti ............. II

285

circumcinctus........... II

273

crudelis ............... II

327

furcatus ............. II

327

inca................. II

331

intrepidus.............. II

329

leucotis................ II

326

melancholicus............. II

$32 \%$

niveigularis............ II

329

nunciolus............. II

332

pipiri................ II

329

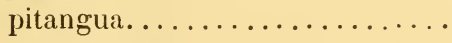

294

rufescens.............. II

372

rutiventris ............. II

187

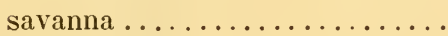

II

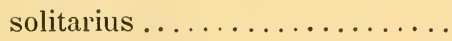

sulphuratus.

332

288

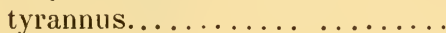

II

286

varius $\ldots \ldots \ldots \ldots \ldots$ II

332

violentus................ II

326

332

ynca................ II

331

U

Uncirostrum cyaneum............. I

Upucerthia atacamensis........... II

Jelskii............... II

montana .............. II

108

nigrofumosa............ II

110

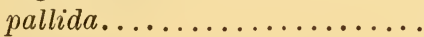

107

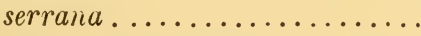

Urallia cissiura

II

107

329

Uranomitra cyanicollis............... I

397

Urax erythrorhynchus............ III

$26 \pi$

mitu

III

Urax (Nothocrax) urumutum

III

265 
Urochroma Hueti................ Tomes III

Urogalba Amazonum..... .......... . III

Pages 217

paradisea Amazonum......... III

Urospatha Martii................ III

Urosticte intermedia................

Urubitinga anthracina... . . ........

brasiliensis .................

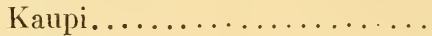

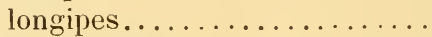

meridionalis ..............

schistacea...............

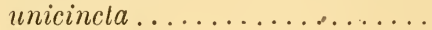

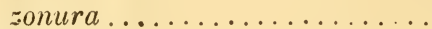

Urubitornis solitaria................

Vanellus cayennensis............ III

chilensis . . . . . . . . . . . .

griseus..................

helveticus ................

melanogaster...............

occidentalis................

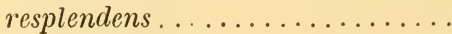

336

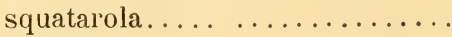

338

varius.

338

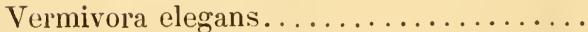

Vireo flavoviridis .................

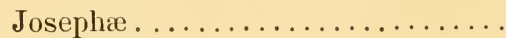

olivacea..................

versicolor .................

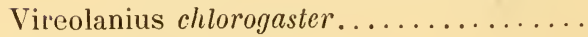

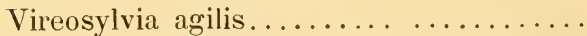

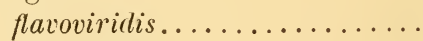

Josepha...............

olivacea.

445

444

443

366

447

443

445

Volatinia.........................

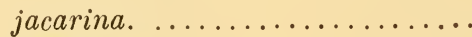

Vultur atratus

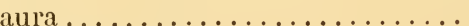

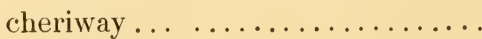

condor

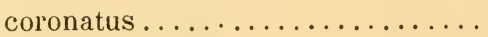

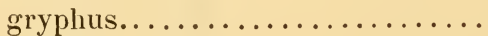

harpyia...$\ldots \ldots \ldots \ldots \ldots$ 
Vultur iota.................. Tomes I

jota................... II

Pages 84

magellanicus ............. [

551

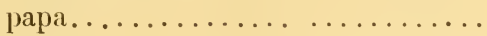

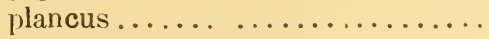

urubu................. I

\section{$\mathbf{X}$}

Xanthornus chrysocarpus........... II

chrysocephalus...........

416

decumanus..............

II

II

404

leucorhamphus ..........

II

413

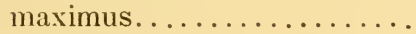

II

104

mesomelas ..............

417

purpurascens.............

II

422

Xanthosomus icterocephalus.............

II

425

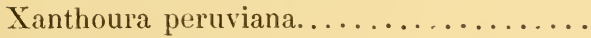

II

396

Xanthura joliæa...................

II

401

peruviana ..............

II

II

396

viridicyanea ..............

II

400

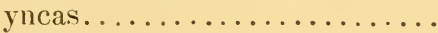

II

396

Xema atricilla....................

III

450

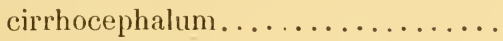

III

collaris...................

III

furcatum

III

furcatus...................

III

Sabinei................. III

Sabini.................. . III

III

Sabinii.................. III

Xenicopsis rufosuperciliata...........

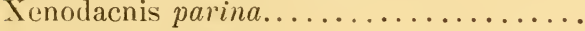

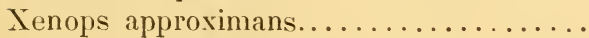

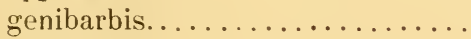

heterurus . ................

littoralis ................

rufosuperciliatus.............

rutilans ...................

rutilus.

Xenospingus concolor................

Xiphocolaptes compressirustris..........

promeropirhynchus......

Xiphorhynchus thoracicus............

Xylocota Jamesoni. 
z

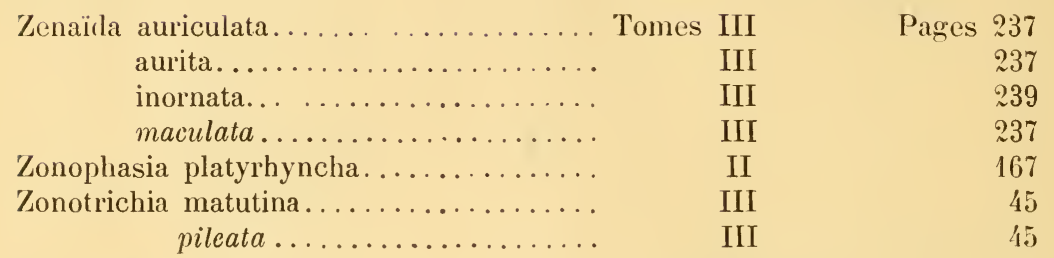








\section{S.}

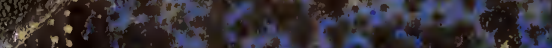

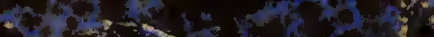

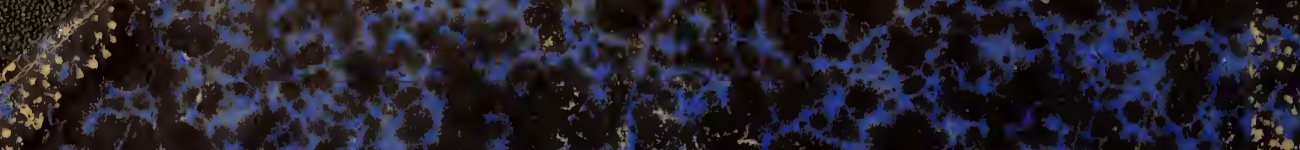

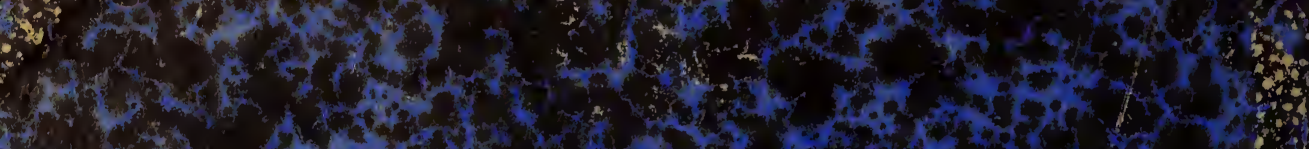

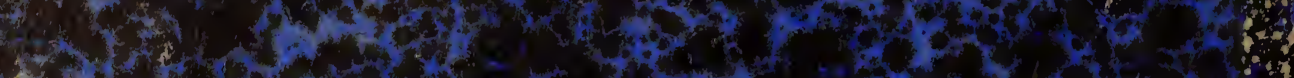

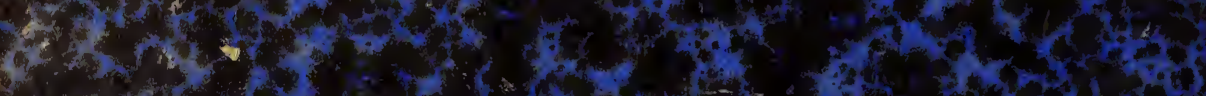
(6)

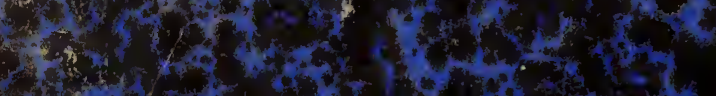

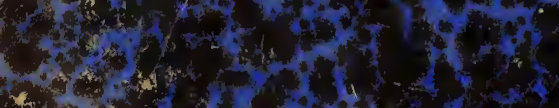

$x_{1}+x_{2}+4$

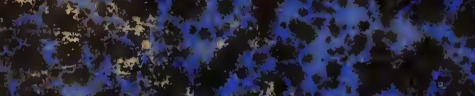

$x_{-\infty} x^{2}+x^{2}+3$

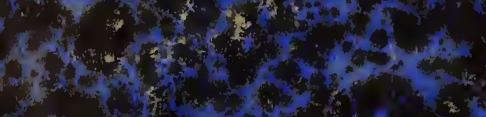

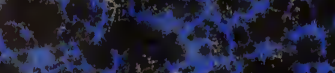

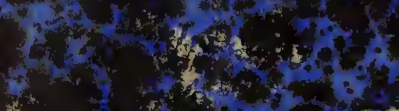

$x^{2}+y^{2}=5^{2}$

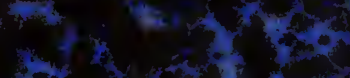

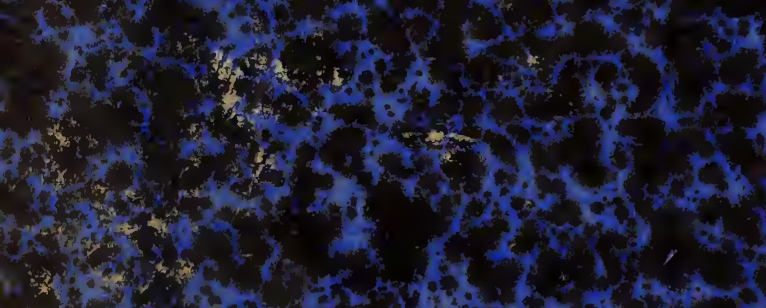

(3)

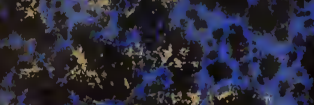

wit.

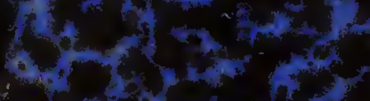

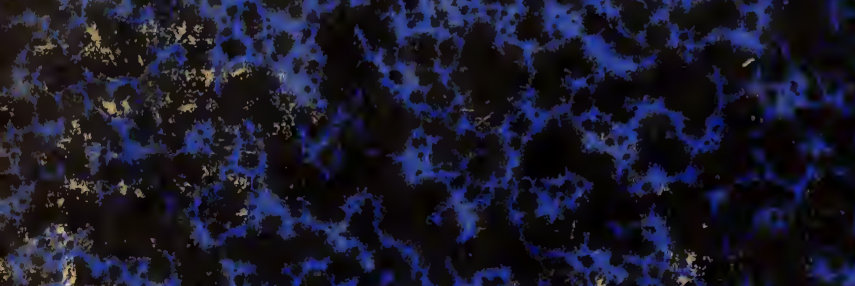

(5)

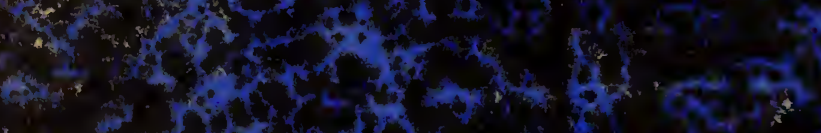

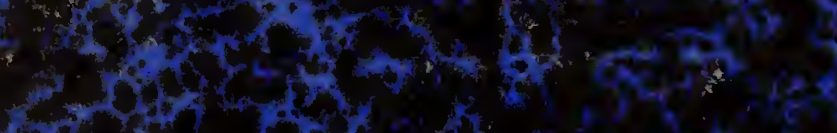

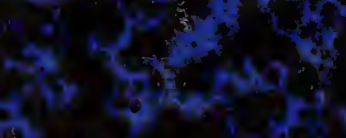

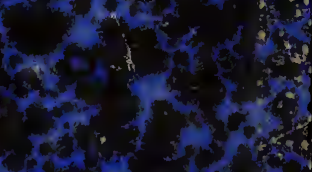

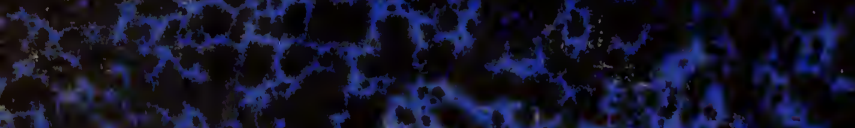

a

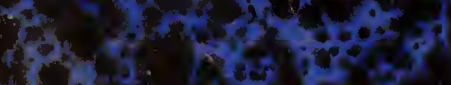

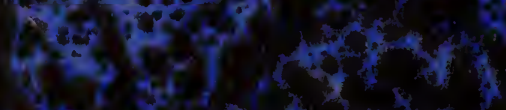

(1)

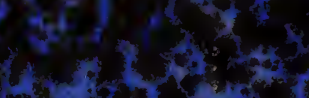

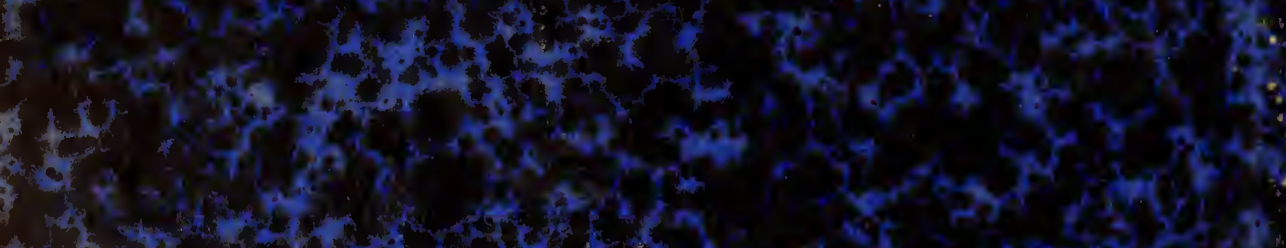

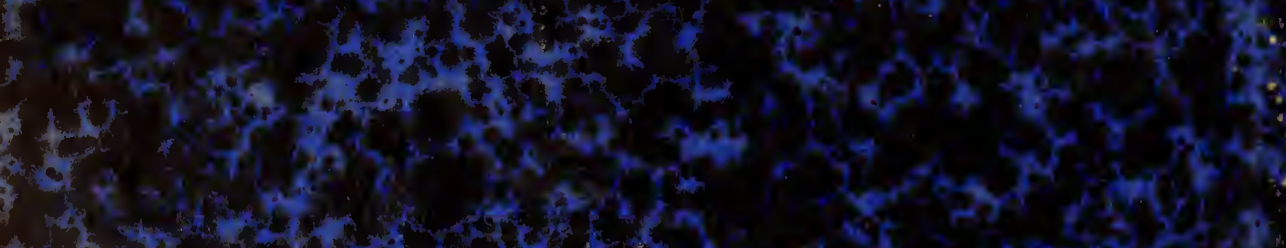

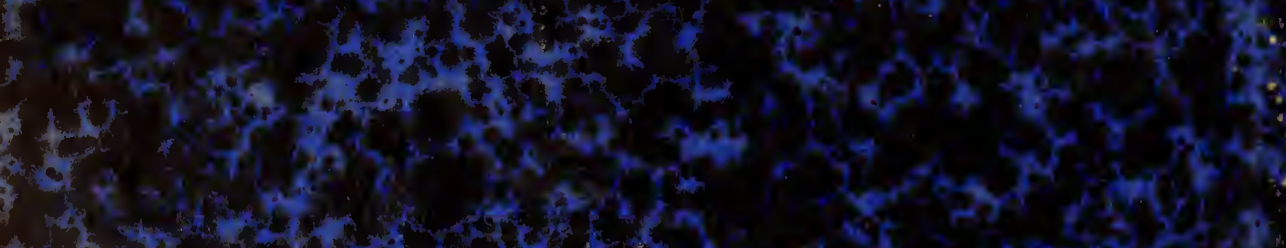

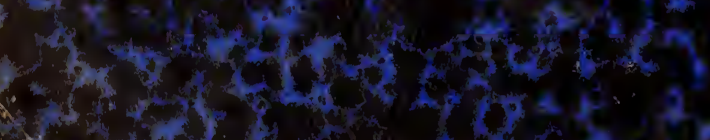

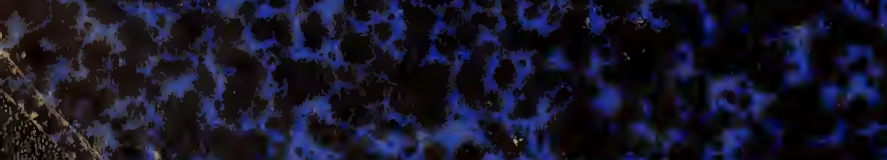

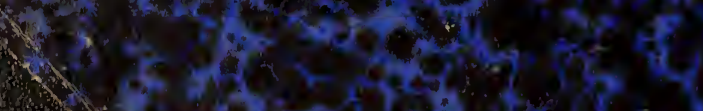

exiative

: 8

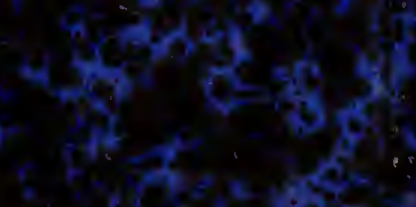

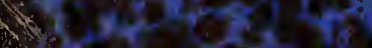

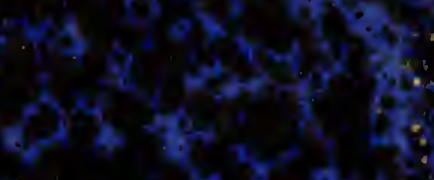

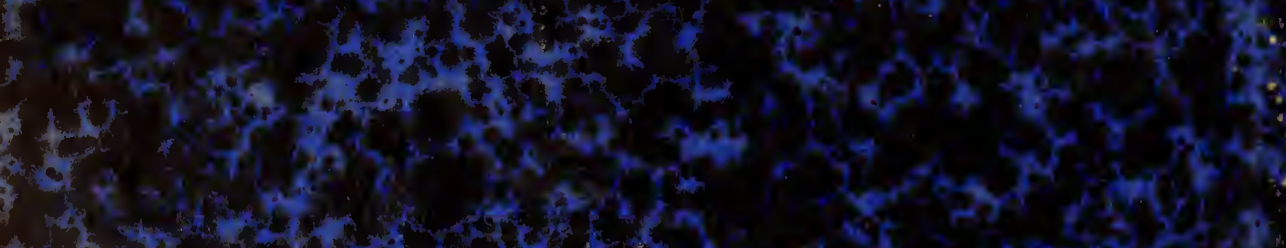

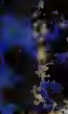

